\title{
Subcriticality Measurements with HEU (93.2) Metal Annular Storage Castings
}

\section{September 2007}

\author{
Prepared by \\ John T. Mihalczo \\ Work completed by \\ James J. Henkel \\ Michael C. Wright \\ Daniel E. Archer \\ John T. Mihalczo \\ James A. Mullens
}




\section{DOCUMENT AVAILABILITY}

Reports produced after January 1, 1996, are generally available free via the U.S. Department of Energy (DOE) Information Bridge.

Web site http://www.osti.gov/bridge

Reports produced before January 1, 1996, may be purchased by members of the public from the following source.

National Technical Information Service

5285 Port Royal Road

Springfield, VA 22161

Telephone 703-605-6000 (1-800-553-6847)

TDD 703-487-4639

Fax 703-605-6900

E-mail info@ntis.gov

Web site http://www.ntis.gov/support/ordernowabout.htm

Reports are available to DOE employees, DOE contractors, Energy Technology Data Exchange (ETDE) representatives, and International Nuclear Information System (INIS) representatives from the following source.

Office of Scientific and Technical Information

P.O. Box 62

Oak Ridge, TN 37831

Telephone 865-576-8401

Fax 865-576-5728

E-mail reports@osti.gov

Web site http://www.osti.gov/contact.html

This report was prepared as an account of work sponsored by an agency of the United States Government. Neither the United States government nor any agency thereof, nor any of their employees, makes any warranty, express or implied, or assumes any legal liability or responsibility for the accuracy, completeness, or usefulness of any information, apparatus, product, or process disclosed, or represents that its use would not infringe privately owned rights. Reference herein to any specific commercial product, process, or service by trade name, trademark, manufacturer, or otherwise, does not necessarily constitute or imply its endorsement, recommendation, or favoring by the United States Government or any agency thereof. The views and opinions of authors expressed herein do not necessarily state or reflect those of the United States Government or any agency thereof. 


\title{
SUBCRITICALITY MEASUREMENTS WITH HEU (93.2) METAL ANNULAR STORAGE CASTINGS
}

\author{
Prepared by \\ John T. Mihalczo \\ Oak Ridge National Laboratory \\ Work completed by \\ James J. Henkel \\ Michael C. Wright \\ Daniel E. Archer \\ John T. Mihalczo \\ James A. Mullens
}

Date Published: September 2007

Prepared by

OAK RIDGE NATIONAL LABORATORY

Oak Ridge, Tennessee 37831-6285

managed by

UT-BATTELLE, LLC

for the

U.S. DEPARTMENT OF ENERGY

under contract DE-AC05-00OR22725 



\section{CONTENTS}

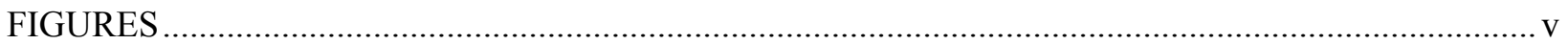

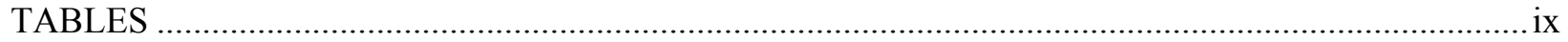

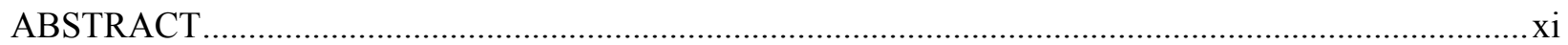

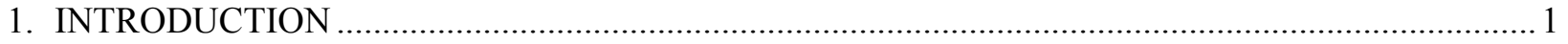

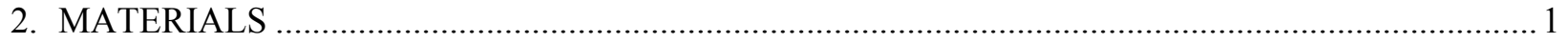

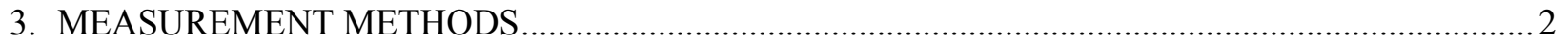

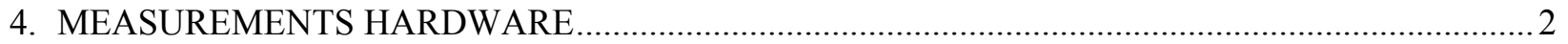

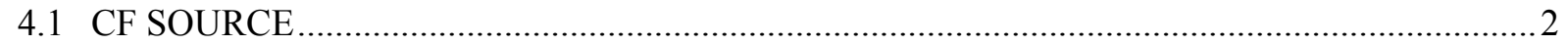

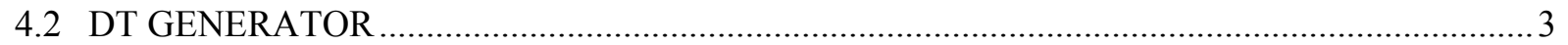

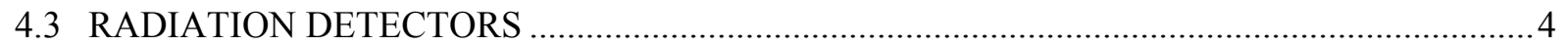

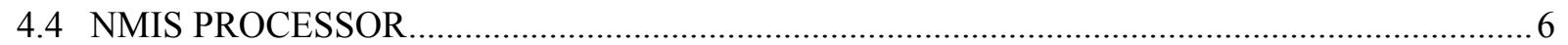

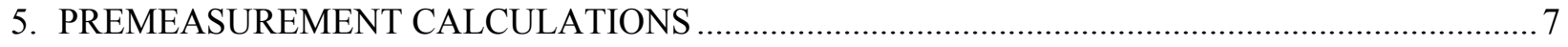

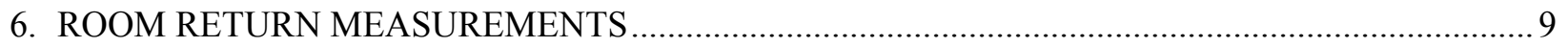

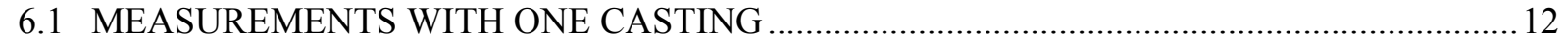

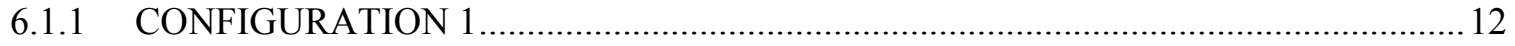

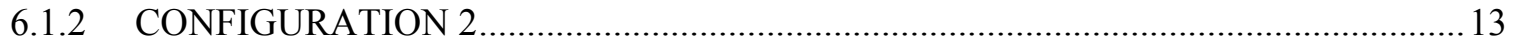

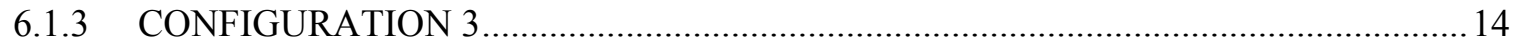

6.1.4 COMPARISON OF MEASUREMENTS WITH THE TWO SOURCES ….................. 16

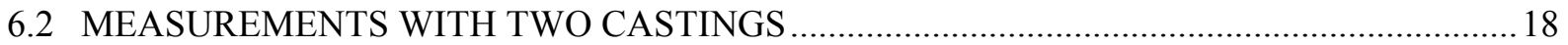

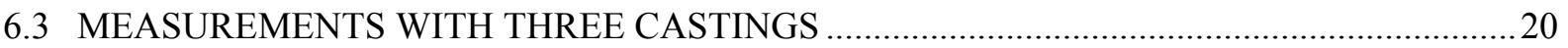

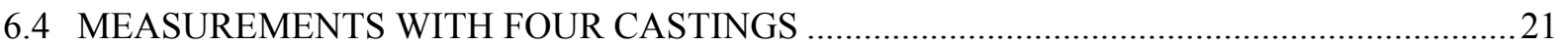

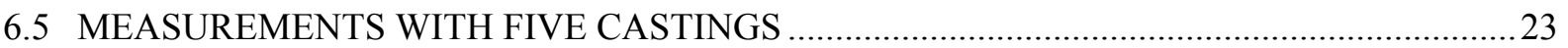

6.6 COMPARISON OF MEASUREMENTS FOR DIFFERENT CASTINGS ...............................2

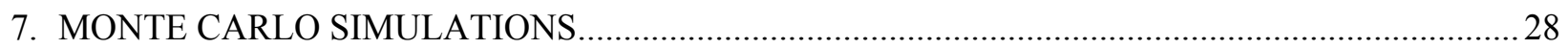

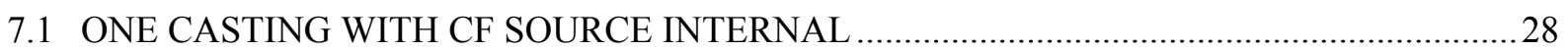

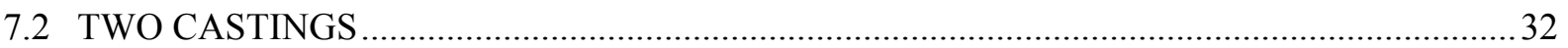

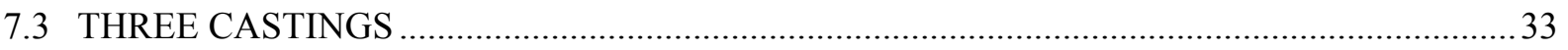

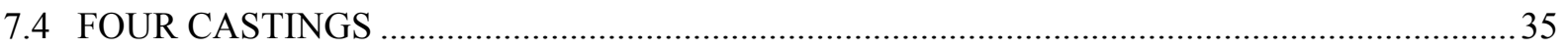

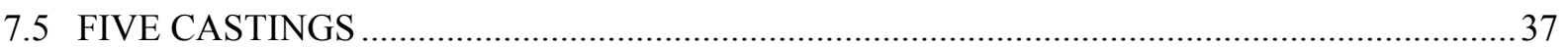

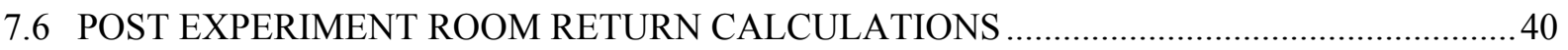

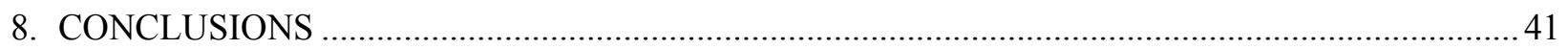

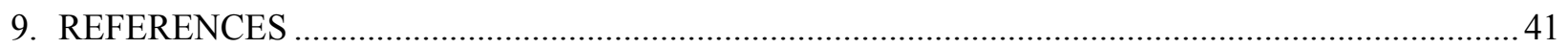

APPENDIX A: URANIUM ISOTOPICS AND IMPURITY ANALYSESError! Bookmark not defined. APPENDIX B: DESCRIPTIONS OF THE CANS FOR THE CASTINGS …......................................... 48

APPENDIX C: SOURCE-DETECTOR-CASTING CONFIGURATIONS .......................................... 54

APPENDIX D: GAPS BETWEEN CASTINGS CANS FOR VARIOUS SOURCE-DETECTOR-

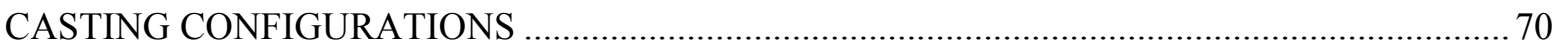

APPENDIX E: ADDITIONAL COMPARISONS OF MEASUREMENT AND MONTE CARLO

CALCULATIONS

APPENDIX F: MONTE CARLO INPUTS FOR SOME SOURCE DETECTOR CASTING

CALCULATIONS 162 



\section{FIGURES}

Figure Page

4.1. Photograph of the $\mathrm{Cf}$ source mounted on ionization chamber......................................................... 3

4.2. The DT generator with an ORTEC base and PMT for alpha detection attached............................... 4

4.3. Neutron counts as a function of angle across the slit aperture in the $11-\mathrm{mm}$ vertical direction. ........... 4

4.4. Neutron counts as function of angle across the slit aperture in the 52-mm horizontal direction.......... 4

4.5. Typical time distributions of neutrons after $\mathrm{Cf}$ fission to determine the detection efficiency as a

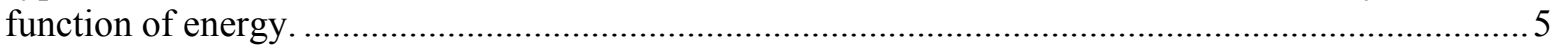

4.6. Typical detection efficiency as a function of neutron energy..................................................... 6

5.1. Calculated time distribution of fission as a function of time after $\mathrm{Cf}$ fission with the source

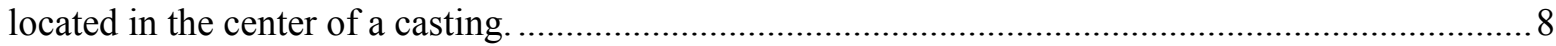

5.2. Time distribution of fission after a previous fission as a function of the number of castings. .............8

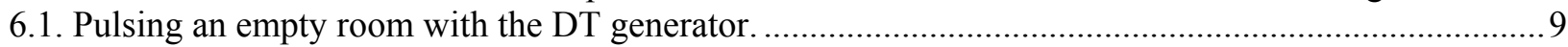

6.2. Room return from $\mathrm{Cf}$ with table top at $40 \mathrm{in}$. from the floor. ...................................................... 10

6.3. Time distribution for all four detectors from Fig. 4.4 averaged over nine channels and for longer

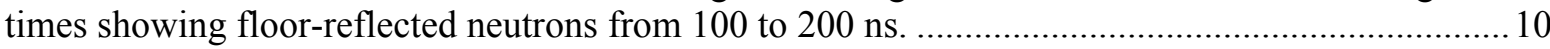

6.4. Cf source and no castings with the table 29 in. above floor. ............................................................ 11

6.5. Comparison of the measurements with $\mathrm{Cf}$ and no castings with the measurements

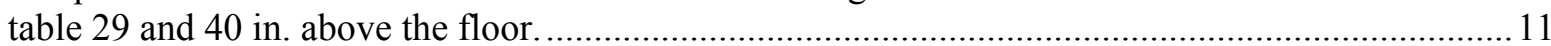

6.6. The Cf source-detector-single uranium metal casting with the Cf source on the axis of the casting. . 12

6.7. Time distribution of coincidence count per $\mathrm{Cf}$ fission between each of the four detectors and the $\mathrm{Cf}$ source fission with the $\mathrm{Cf}$ source on the axis of the annular casting.......................................... 13

6.8. Time distribution of coincidences between detectors with the $\mathrm{Cf}$ source internal........................... 13

6.9. Time distribution of coincidence count per $\mathrm{Cf}$ fission between each of the three detectors and the $\mathrm{Cf}$

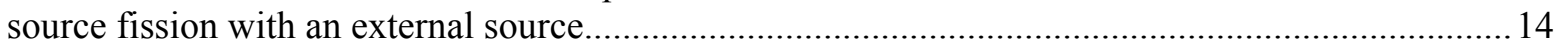

6.10. Time distribution of coincidences between three detectors with an external $\mathrm{Cf}$ source.................... 14

6.11. Time distribution of coincidence count per tagged-source neutron between each of the three detectors and the DT generator neutrons with the source external. ............................................... 15

6.12. Time distribution of coincidences between detectors for measurements with an external DT source.

6.13. Comparison of the time distribution of counts in one detector (2) with respect to the source event for the three measurement configurations....

6.14. The time distribution of coincidences between detectors on opposite sides of the casting for the three configurations

6.15. Photograph of $\mathrm{Cf}$ source-detector-casting configuration with the $\mathrm{Cf}$ source internal for a measurement with two castings.

6.16. The time distribution of counts in each of the detectors after $\mathrm{Cf}$ fission for configuration four of two castings with the $\mathrm{Cf}$ source internal.

6.17. Time distribution of counts in one detector with respect to a previous count in another detector for configuration 4 of two castings with the $\mathrm{Cf}$ source internal to one.

6.18. Time distribution of count in the detectors after $\mathrm{Cf}$ fission for configuration 12 (three castings with $\mathrm{Cf}$ internal).

6.19. Time distribution of counts in one detector with respect to a previous count in another detector.....21

6.20. Photograph of the DT source-detector-casting configuration 20 for measurement with four castings.

6.21. Time distribution of count in the detectors after $\mathrm{Cf}$ fission for configuration 20

(four castings with $\mathrm{Cf}$ internal).

6.22. Time distribution of counts in one detector with respect to a previous count in another detector for configuration 20 for all detector combinations. 
6.23. Photograph of one of the DT source-detector-configurations for a measurement with five castings.

6.24. Time distribution of count in the detectors after $\mathrm{Cf}$ fission for configuration 20

(four castings with $\mathrm{Cf}$ internal).

6.25. Time distribution of counts in one detector with respect to a previous count in another detector for configuration 26.

6.26. The time distribution of the sum of all detector counts (M) after $\mathrm{Cf}$ fission.

6.27. The time distribution of the sum of all detector counts (M) after $\mathrm{Cf}$ fission between 350 and $500 \mathrm{~ns}$.

6.28. The time distribution of the sum of all detector counts $(\mathrm{M})$ after $\mathrm{Cf}$ fission for the first $125 \mathrm{~ns}$.......27

6.29. Time distribution of counts after DT reaction summed over all detectors after $\mathrm{Cf}$ fission for none and 1 to 5 casting.

6.30. Time distribution of counts after DT reaction summed over all detectors after Cf fission

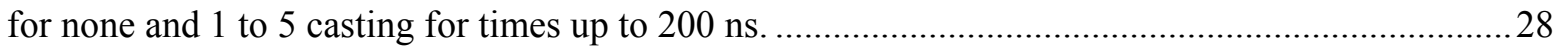

7.1. Top view Monte Carlo geometry for calculations of a single casting with the Cf source internal.....29

7.2. Time distribution of counts in detector 1 after $\mathrm{Cf}$ fission in counts per $\mathrm{Cf}$ fission...........................29

7.3. Time distribution of counts in detector.

7.4. Time distribution of counts in detector 1 after Cf fission in counts per Cf fission for the first $200 \mathrm{~ns}$. Blue curve is measured and the other is calculated. 31

7.5. Time distribution of counts in detector 1 after $\mathrm{Cf}$ fission in counts per $\mathrm{Cf}$ fission...........................3 31

7.6. Top view Monte Carlo geometry for calculations of a two casting with the Cf source internal. ......... 32

7.7. Time distribution of counts in detector 1 after $\mathrm{Cf}$ fission in counts per $\mathrm{Cf}$ fission............................33

7.8. Top view Monte Carlo geometry for calculations of a three casting with the Cf source internal......... 34

7.9. Time distribution of counts in detector 3 after $\mathrm{Cf}$ fission in counts per $\mathrm{Cf}$ fission............................34

7.10. Time distribution of counts in detector 4 after $\mathrm{Cf}$ fission in counts per $\mathrm{Cf}$ fission............................35

7.11. Top view Monte Carlo geometry for calculations of four casting with the Cf source internal...........36

7.12. Time distribution of counts in detector 1 after $\mathrm{Cf}$ fission in counts per $\mathrm{Cf}$ fission.............................36

7.13. Time distribution of counts in detector 2 after $\mathrm{Cf}$ fission in counts per Cf fission......................... 37

7.14. Top view Monte Carlo geometry for calculations of five castings with the $\mathrm{Cf}$ source internal. ....... 38

7.15. Time distribution of counts in detector 1 after $\mathrm{Cf}$ fission in counts per Cf fission..........................38

7.16. Time distribution of counts in detector 3 after $\mathrm{Cf}$ fission in counts per $\mathrm{Cf}$ fission..........................39

7.17. Time distribution of counts in detector 2 for $200 \mathrm{~ns}$ after $\mathrm{Cf}$ fission in counts per Cf fission...........39

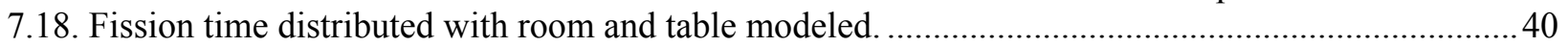

7.19. Percent difference between model with room/table modeled and without room/table modeled. ...... 41

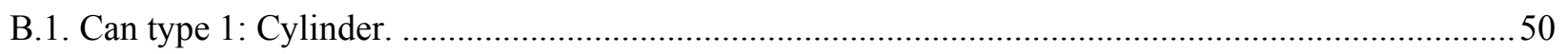

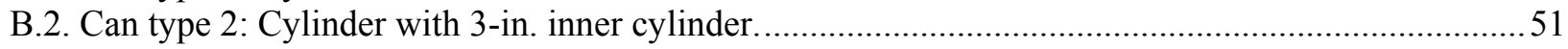

B.3. Can type 2: Cylinder with two welded trunnions and a cable wire for carrying can. ........................52

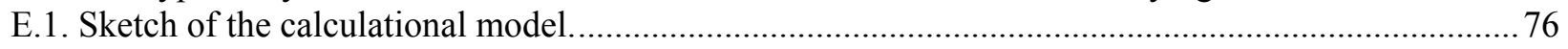

E.2. Time distribution of counts in detector 1 after $\mathrm{Cf}$ fission for $512 \mathrm{~ns}$ for configuration 1.................77

E.3. Time distribution of counts in detector 1 for after $\mathrm{Cf}$ fission for $200 \mathrm{~ns}$ for configuration 1 ..............78

E.4. Time distribution of counts in detector 1 after $\mathrm{Cf}$ fission for $30 \mathrm{~ns}$ for configuration 1 ...................79

E.5. Time distribution of counts in detector 2 after $\mathrm{Cf}$ fission for $512 \mathrm{~ns}$ for configuration 1................. 80

E.6. Time distribution of counts in detector 2 after $\mathrm{Cf}$ fission for $200 \mathrm{~ns}$ for configuration 1 ................8 81

E.7. Time distribution of counts in detector 2 after $\mathrm{Cf}$ fission for $30 \mathrm{~ns}$ for configuration 1 ..................8 82

E.8. Time distribution of counts in detector 3 after $\mathrm{Cf}$ fission for $512 \mathrm{~ns}$ for configuration 1.................83

E.9. Time distribution of counts in detector 3 after $\mathrm{Cf}$ fission for $200 \mathrm{~ns}$ for configuration 1................84

E.10. Time distribution of counts in detector 3 after $\mathrm{Cf}$ fission for $30 \mathrm{~ns}$ for configuration 1.................85

E.11. Time distribution of counts in detector 4 after $\mathrm{Cf}$ fission for $512 \mathrm{~ns}$ for configuration 1...............86

E.12. Time distribution of counts in detector 1 after $\mathrm{Cf}$ fission for $200 \mathrm{~ns}$ for configuration 1................87

E.13. Time distribution of counts in detector 1 after $\mathrm{Cf}$ fission for $30 \mathrm{~ns}$ for configuration 1 ..................8 88

E.14. Time distribution of counts in detectors 3 after a count in detector 1 for $512 \mathrm{~ns}$ 
for configuration 1

E.15. Time distribution of counts in detectors 3 after a count in detector 1 for $200 \mathrm{~ns}$ for configuration 1 .

E.16. Time distribution of counts in detectors 3 after a count in detector 1 for $30 \mathrm{~ns}$ for configuration 1 . 91

E.17. Time distribution of counts in detector 1 after $\mathrm{Cf}$ fission for $512 \mathrm{~ns}$ for configuration 3 . .992

E.18. Time distribution of counts in detector 1 after $\mathrm{Cf}$ fission for $200 \mathrm{~ns}$ for configuration 3.

E.19. Time distribution of counts in detector 3 after $\mathrm{Cf}$ fission for $30 \mathrm{~ns}$ for configuration 3 .

E.20. Time distribution of counts in detector 2 after $\mathrm{Cf}$ fission for $512 \mathrm{~ns}$ for configuration 3 .

E.21. Time distribution of counts in detector 2 after $\mathrm{Cf}$ fission for $200 \mathrm{~ns}$ for configuration 3.

E.22. Time distribution of counts in detector 2 after $\mathrm{Cf}$ fission for $30 \mathrm{~ns}$ for configuration 3.

E.23. Time distribution of counts in detector 3 after $\mathrm{Cf}$ fission for $512 \mathrm{~ns}$ for configuration 3.

E.24. Time distribution of counts in detector 3 after $\mathrm{Cf}$ fission for $200 \mathrm{~ns}$ for configuration 3.

E.25. Time distribution of counts in detector 3 after $\mathrm{Cf}$ fission for $30 \mathrm{~ns}$ for configuration 3 .

E.26. Time distribution of counts in detector 1 after $\mathrm{Cf}$ fission for $512 \mathrm{~ns}$ for configuration 4.

E.27. Time distribution of counts in detector 1 after $\mathrm{Cf}$ fission for $200 \mathrm{~ns}$ for configuration 4.

E.28. Time distribution of counts in detector 1 after $\mathrm{Cf}$ fission for $30 \mathrm{~ns}$ for configuration 4.

E.29. Time distribution of counts in detector 2 after $\mathrm{Cf}$ fission for $512 \mathrm{~ns}$ for configuration 4.

E.30. Time distribution of counts in detector 2 after $\mathrm{Cf}$ fission for $200 \mathrm{~ns}$ for configuration 4.

E.31. Time distribution of counts in detector 2 after $\mathrm{Cf}$ fission for $30 \mathrm{~ns}$ for configuration 4 .

E.32. Time distribution of counts in detector 3 after $\mathrm{Cf}$ fission for $512 \mathrm{~ns}$ for configuration 3.

E.33. Time distribution of counts in detector 3 after $\mathrm{Cf}$ fission for $200 \mathrm{~ns}$ for configuration 4.

E.34. Time distribution of counts in detector 3 after $\mathrm{Cf}$ fission for $30 \mathrm{~ns}$ for configuration 4 ................

E.35. Time distribution of counts in detector 4 after $\mathrm{Cf}$ fission for $512 \mathrm{~ns}$ for configuration 4.

E.36. Time distribution of counts in detector 4 after $\mathrm{Cf}$ fission for $200 \mathrm{~ns}$ for configuration 4 .

E.37. Time distribution of counts in detector 4 after $\mathrm{Cf}$ fission for $30 \mathrm{~ns}$ for configuration 4.

E.38. Time distribution of counts in detector 3 after a count in detector 1 for $512 \mathrm{~ns}$ for configuration 4

E.39. Time distribution of counts in detector 3 after a count in detector 1 for $200 \mathrm{~ns}$ for configuration 4

E.40. Time distribution of counts in detector 3 after a count in detector 1 for $40 \mathrm{~ns}$ for configuration 4 .......

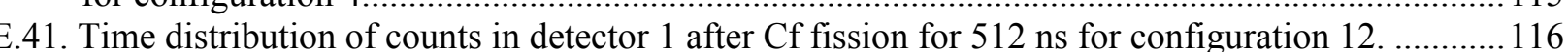

E.42. Time distribution of counts in detector 1 after $\mathrm{Cf}$ fission for $200 \mathrm{~ns}$ for configuration 12.............117

E.43. Time distribution of counts in detector 1 after $\mathrm{Cf}$ fission for $30 \mathrm{~ns}$ for configuration 12 .............. 118

E.44. Time distribution of counts in detector 2 after Cf fission for 512 ns for configuration 12........... 119

E.45. Time distribution of counts in detector 2 after Cf fission for $200 \mathrm{~ns}$ for configuration 12............ 120

E.46. Time distribution of counts in detector 2 after $\mathrm{Cf}$ fission for $30 \mathrm{~ns}$ for configuration $12 \ldots \ldots \ldots \ldots . . .121$

E.47. Time distribution of counts in detector 3 after $\mathrm{Cf}$ fission for $512 \mathrm{~ns}$ for configuration 12............122

E.48. Time distribution of counts in detector 3 after $\mathrm{Cf}$ fission for $200 \mathrm{~ns}$ for configuration 12............ 123

E.49. Time distribution of counts in detector 3 after $\mathrm{Cf}$ fission for $30 \mathrm{~ns}$ for configuration 12............. 124

E.50. Time distribution of counts in detector 4 after $\mathrm{Cf}$ fission for $512 \mathrm{~ns}$ for configuration 12............ 125

E.51. Time distribution of counts in detector 4 after Cf fission for $200 \mathrm{~ns}$ for configuration 12............126

E.52. Time distribution of counts in detector 4 after $\mathrm{Cf}$ fission for $30 \mathrm{~ns}$ for configuration 12.............. 127

E.53. Time distribution of counts in detector 3 after a count in detector 1 for $512 \mathrm{~ns}$

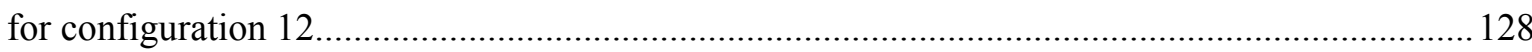

E.54. Time distribution of counts in detector 3 after a count in detector 1 for $200 \mathrm{~ns}$ for configuration 12 .

E.55. Time distribution of counts in detector 3 after a count in detector 1 for $40 \mathrm{~ns}$ for configuration 12

E.56. Time distribution of counts in detector 1 after $\mathrm{Cf}$ fission for $512 \mathrm{~ns}$ for configuration $20 \ldots \ldots \ldots . . . .131$ 
E.57. Time distribution of counts in detector 1 after $\mathrm{Cf}$ fission for $200 \mathrm{~ns}$ for configuration 20............ 132

E.58. Time distribution of counts in detector 1 after $\mathrm{Cf}$ fission for $30 \mathrm{~ns}$ for configuration $20 \ldots \ldots \ldots \ldots . . .133$

E.59. Time distribution of counts in detector 2 after $\mathrm{Cf}$ fission for $512 \mathrm{~ns}$ for configuration 20...........134

E.60. Time distribution of counts in detector 2 after $\mathrm{Cf}$ fission for $200 \mathrm{~ns}$ for configuration 20...........135

E.61. Time distribution of counts in detector 2 after $\mathrm{Cf}$ fission for $512 \mathrm{~ns}$ for configuration 30........... 136

E.62. Time distribution of counts in detector 3 after $\mathrm{Cf}$ fission for $512 \mathrm{~ns}$ for configuration 20........... 137

E.63. Time distribution of counts in detector 3 after $\mathrm{Cf}$ fission for $200 \mathrm{~ns}$ for configuration 20............138

E.64. Time distribution of counts in detector 3 after $\mathrm{Cf}$ fission for $30 \mathrm{~ns}$ for configuration 3............... 139

E.65. Time distribution of counts in detector 4 after $\mathrm{Cf}$ fission for $512 \mathrm{~ns}$ for configuration 20 ............140

E.66. Time distribution of counts in detector 4 after $\mathrm{Cf}$ fission for $200 \mathrm{~ns}$ for configuration 20............141

E.67. Time distribution of counts in detector 4 after $\mathrm{Cf}$ fission for $30 \mathrm{~ns}$ for configuration 20.............. 142

E.68. Time distribution of counts in detector 4 after a count in detector 1 for $512 \mathrm{~ns}$

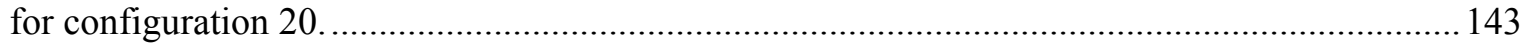

E.69. Time distribution of counts in detector 4 after a count in detector 1 for $200 \mathrm{~ns}$ for configuration 20 .

E.70. Time distribution of counts in detector 4 after a count in detector 1 for $40 \mathrm{~ns}$ for configuration 20

E.71. Time distribution of counts in detector 1 after $\mathrm{Cf}$ fission for $512 \mathrm{~ns}$ for configuration 26........... 146

E.72. Time distribution of counts in detector 1 after $\mathrm{Cf}$ fission for $200 \mathrm{~ns}$ for configuration 26............ 147

E.73. Time distribution of counts in detector 1 after $\mathrm{Cf}$ fission for $30 \mathrm{~ns}$ for configuration 26............. 148

E.74. Time distribution of counts in detector 2 after $\mathrm{Cf}$ fission for $512 \mathrm{~ns}$ for configuration 26............149

E.75. Time distribution of counts in detector 2 after $\mathrm{Cf}$ fission for $200 \mathrm{~ns}$ for configuration 26........... 150

E.76. Time distribution of counts in detector 2 after $\mathrm{Cf}$ fission for $30 \mathrm{~ns}$ for configuration 26............. 151

E.77. Time distribution of counts in detector 3 after $\mathrm{Cf}$ fission for $512 \mathrm{~ns}$ for configuration $26 . \ldots \ldots \ldots . . .152$

E.78. Time distribution of counts in detector 3 after $\mathrm{Cf}$ fission for $200 \mathrm{~ns}$ for configuration 26...........153

E.79. Time distribution of counts in detector 3 after $\mathrm{Cf}$ fission for $30 \mathrm{~ns}$ for configuration $26 \ldots \ldots \ldots \ldots . . .154$

E.80. Time distribution of counts in detector 4 after $\mathrm{Cf}$ fission for $512 \mathrm{~ns}$ for configuration $26 . \ldots \ldots \ldots . . .155$

E.81. Time distribution of counts in detector 4 after $\mathrm{Cf}$ fission for $200 \mathrm{~ns}$ for configuration $26 . \ldots \ldots \ldots . . .156$

E.82. Time distribution of counts in detector 4 after $\mathrm{Cf}$ fission for $30 \mathrm{~ns}$ for configuration 26............. 157

E.83. Time distribution of counts in detector 4 after a count in detector 1 for $512 \mathrm{~ns}$

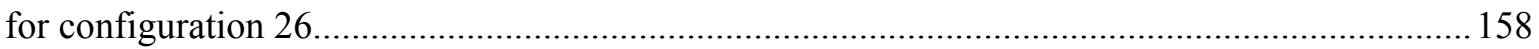

E.84. Time distribution of counts in detector 1 and 4 after $\mathrm{Cf}$ fission for $512 \mathrm{~ns}$ for configuration $26 \ldots 159$

E.85. Time distribution of counts in detector 4 after a count in detector 1 for $40 \mathrm{~ns}$ for configuration 26 


\section{TABLES}

Table

Page

2.1. Description of the uranium metal castings ................................................................................2

4.1. Maximum neutron detection efficiency (\%) and energy threshold (MeV) for neutron energy detection for the various days of operation

5.1. $\mathrm{K}_{\text {eff }}$ values for the bare castings 7 



\begin{abstract}
These carefully performed and documented measurements with unreflected and unmoderated highly enriched uranium (HEU) castings can be used to benchmark calculational methods for the time decay of the fission chain multiplication process as measured with small $(1 \times 1 \times 6$ in. thick plastic scintillators with $1 / 4$-in.-thick lead on all detector surfaces) detectors adjacent to the tightly fitting stainless steel cans that contained the HEU ( $\sim 93 \mathrm{wt} \%)$ metal. Prompt time decay measurements were performed stimulating the fission chain multiplication process with a timed, tagged $\mathrm{Cf}$ spontaneous fission source that emitted fission-spectrum neutrons and a time and directionally tagged 14.1-MeV neutrons from the DT reaction in a steady state generator with an embedded alpha detector. Time decay measurements were performed with HEU masses varying from 18 to $90 \mathrm{~kg}$ for a wide variety of source-detector-casting configurations. The use of a DT generator provided no addition information about the fission chain behavior beyond that provided by a time-tagged $\mathrm{Cf}$ spontaneous fission source. The main quantities obtained in the measurements were (1) the time distribution of the counts in a detector after a neutron fission in the Cf source or after the alpha detection coincident with the emission of a neutron from the DT generator (the equivalent of a pulsed neutron measurement with a randomly pulsed source) and (2) the time distribution of counts in one detector after a count in another detector (the equivalent of a two-detector Rossi-alpha measurement). Monte Carlo calculations using the MCNP-PoliMi coupled gamma-neutron transport code generally agreed with the measurement results except for some differences early in the fission chain decay process. The measurements that were performed with the HEU about $1 \mathrm{~m}$ above the floor were considerably affected by room return neutrons at times as early as $100 \mathrm{~ns}$, and at times after $300 \mathrm{~ns}$, a major portion of the time response was associated with the interaction of the HEU assemblies with the floor. This room-return effect increased with the size of the assembly because the larger assemblies subtend a larger solid angle to a neutron returning from the floor.
\end{abstract}





\section{INTRODUCTION}

A series of experiments with annular highly enriched uranium (HEU) metal castings was performed in 2006 in which up to five castings were assembled in a tightly packed array with minimal spacing between castings. The castings were the standard HEU storage castings used at the Y-12 National Security Complex and were in stainless steel cans for contamination control. Each can contained slightly less than $18 \mathrm{~kg}$ of uranium with $93.16 \mathrm{wt} \%{ }^{235} \mathrm{U}$. The fission chain multiplication process was initiated by a nearby ${ }^{252} \mathrm{Cf}$ spontaneously fissioning neutron source [1] or a small portable DT neutron generator with an embedded alpha detector [2] and the prompt neutron time behavior was measured with small plastic scintillation detectors sensitive to the fast neutrons and gamma rays without distinction using the Nuclear Materials Identification System (NMIS) [3]. The neutron emission from the Cf source was time tagged because the $\mathrm{Cf}$ was deposited on one plate of a parallel plate ionization chamber, and the neutron emission from the DT generator was time and directionally tagged by the embedded alpha detector. These experiments were performed to benchmark methods for the calculation of prompt neutron time behavior. This report describes the measurements and compares the results for both types of neutron sources and compares Monte Carlo calculations with the measured results for selected measurements. This work was supported by the Y-12 National Security Complex and the Department of Energy (DOE) National Nuclear Security Administration.

\section{MATERIALS}

The fissile material for these measurements was highly enriched uranium metal with an average ${ }^{235} \mathrm{U}$ enrichment of $93.158 \mathrm{wt} \%$. The average ${ }^{234} \mathrm{U}$ and ${ }^{236} \mathrm{U}$ content were 1.010 and $0.412 \mathrm{wt} \%$, respectively. The average ${ }^{238} \mathrm{U}$ content is the difference of these numbers from 100. The HEU metal was in the form of the standard HEU annular storage casting at the Y-12 Complex. The outside diameter of the casting was $5.000 \mathrm{in}$., and the inside diameter was $3.500 \mathrm{in}^{1}{ }^{1}$ These dimensions were not measured but were assumed based on the dimensions of the graphite casting crucible and measurements of previous castings of this same type. The height of the castings depended on the masses, which were between 17.636 and $17.996 \mathrm{~kg}$, and the average impurity content was $\sim 992 \mathrm{ppm}$. The results of mass spectrographic analyses for the impurity content and the uranium isotopics are given in Appendix A. The meniscus at the top of the casting that resulted from solidifying of the melt was $1 / 16$ in. deep. It was assumed that the density of the uranium metal is $18.75 \mathrm{gm} / \mathrm{cm}^{3}$. A summary of the description of the HEU metal castings is given in Table 2.1.

The castings were in 0.025-in.-thick stainless steel (SS-304) cylindrical cans for contamination control; the cans were 7.5 in. high. Four of the cans had recessed lids welded into the bottom and had the same type of lid glued into the top. The fifth can was annular in shape had an inside diameter of 3.0 in. so that the $\mathrm{Cf}$ source could be located on the axes of this casting; thus, it had 0.025 -in.-thick stainless steel on the inside of the casting.

The average diameters of the cans with the casting inserted and other details of the castings are given in Table 2.1. The recessed lid welded on the bottom of the cans resulted in the bottom of the uranium metal castings being $0.25 \mathrm{in}$. above the steel table. To preclude water accumulation inside the annular can, this can rested on a 0.25 -in.-thick A36 mild steel coaster that would allow water to drain out. So for the annular casting No. 1 , the bottom of the HEU metal was $\sim 0.50$ in. above the 0.25 -in.-thick steel table, while for castings 2 to 5 , this distance was 0.25 in. Appendix B provides drawings and gives a detailed description of the cans used for castings.

\footnotetext{
${ }^{1}$ All dimensions given in this report are in the units measured.
} 
Table 2.1. Description of the uranium metal castings

\begin{tabular}{ccccccc}
\hline Number & $\begin{array}{c}\text { Uranium } \\
\text { (gm) }\end{array}$ & $\begin{array}{c}\text { Impurity content } \\
\text { (ppm) }\end{array}$ & \multicolumn{3}{c}{$\begin{array}{c}\text { Isotopic content } \\
\text { (wt \%) }\end{array}$} & $\begin{array}{c}\text { Average can diameter } \\
\text { (in.) } \mathbf{b}^{\boldsymbol{b}}\end{array}$ \\
\hline & & & $\mathbf{2 3 4}$ & $\mathbf{2 3 5}$ & $\mathbf{2 3 6}$ & \\
\cline { 5 - 6 } $1^{c}$ & 17920 & 1105 & 1.008 & 93.186 & 0.377 & 5.067 \\
2 & 17996 & 1200 & 1.017 & 93.168 & 0.394 & 5.062 \\
3 & 17636 & 988 & 1.012 & 93.175 & 0.435 & 5.063 \\
4 & 17862 & 816 & 1.009 & 93.177 & 0.451 & 5.064 \\
5 & 17852 & 848 & 1.005 & 93.170 & 0.410 & 5.062 \\
\hline
\end{tabular}

${ }^{a}$ The last four digits of the part numbers were $2811,3007,3546,3014$, and 3480 for the material in cans, respectively. The last four digits of the original part numbers were $0031,005,0015,0042$, and 0039 .

${ }^{b}$ The void space dimensions between the uranium metal and the cans were $0.0085,0.0060,0.0065,0.0070$, and 0.0060 in., respectively

${ }^{c}$ This casting was in an annular can with a inside diameter of $3.0 \mathrm{in}$.

Note: It was assumed that the uranium metal density was $18.75 \mathrm{~g} / \mathrm{cm}^{3}$.

\section{MEASUREMENT METHODS}

Neutrons from the ${ }^{252} \mathrm{Cf}$ spontaneously fissioning neutron source were time tagged by detecting the ionization from the fission product emitted from the $\mathrm{Cf}$ on one plate of a parallel plate ionization chamber. A fraction of the neutron emission from the DT generator was detected by an embedded alpha detector which measured the direction and time of emission of the alpha particle detector. Since the neutron is emitted $\sim 180^{\circ}$ from the alpha particle in the DT reaction, the alpha particle detector can be use to time and directionally tag the neutron emission. Neutrons from the two types of sources initiate fission in the HEU, and four small plastic scintillation detectors were used to measure the time behavior of the radiation emitted in the fission chain decay. The fast $(1 \mathrm{GHz})$ NMIS processor recorded the time distribution of radiation emitted. Several types of measurements were performed: (1) the time distribution of events in a detector with respect to a previous event in the same detector (autocorrelation function), which is the equivalent of the single detector Rossi-alpha measurement; (2) the time distribution of events in one detector with respect to a previous event in another detector (cross correlation function), which is equivalent to the two-detector Rossi-alpha measurements; (3) and the time distribution of events in a detector with respect to the source neutron emission, which is the equivalent of the pulsed neutron measurements. All these provide the time decay of the fission chain population, which usually can be represented by the sum of exponentials for systems with a low neutron multiplication factor. In this work, the prompt decay will not be fitted to obtain this functional form, but the ability of the Monte Carlo method to calculate this time dependence will be determined. Other quantities measured and not presented in this report are the multiplets, which are the number of time $n$ detections are recorded in each, and the sum of all detectors in a time interval and higher-order correlations, which are the time distribution of events in any and all combinations of three detectors. All these quantities are measured simultaneously by the NMIS processor.

\section{MEASUREMENTS HARDWARE}

\subsection{CF SOURCE}

The $\mathrm{Cf}$ source was approximately $0.55 \mu \mathrm{g}$ of ${ }^{252} \mathrm{Cf}$ and has a spontaneous fission rate of 330,000 fissions per second. The time-tagged neutrons from this source are emitted in all directions. One of the castings 
was canned in such a way that the $\mathrm{C}$ f source in its ionization chamber could be place on the axis of the casting. This source was also located in some measurements adjacent to the outer surface of the casting can. A photograph of the Cf source ionization chamber and amplifier electronics is given in Fig 4.1. The spontaneous fission detection system was such that it detected some false triggers from alpha particle detection from the radioactive decay of ${ }^{252} \mathrm{Cf}$. These decays are a factor of $\sim 30$ more numerous than spontaneous fission, and measurements determined that $1 \%$ of the Cf triggers were false and not related to the emission of neutrons. In all measurements, the Cf source was located $3.25 \mathrm{in}$. above the steel table. For internal locations the 1-cm.-diam. Cf deposit was horizontal. For external locations, most of the time the deposit was vertical and adjacent to the surface of a can or in between adjacent cans.

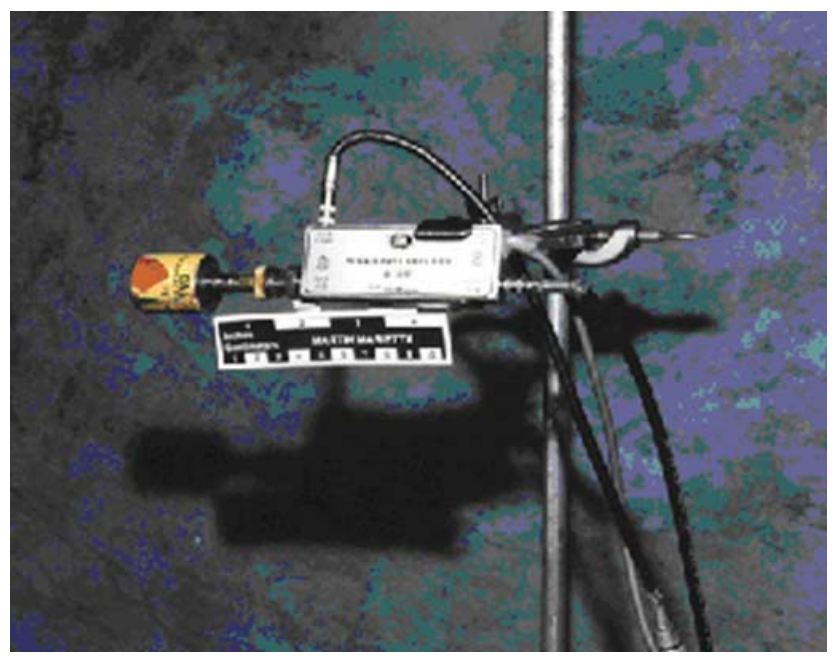

Fig. 4.1. Photograph of the Cf source mounted on ionization chamber.

\subsection{DT GENERATOR}

The small portable DT generator used for the measurements was a Thermo Fischer Scientific Corp. model API120 with an embedded YAP scintillation detector for alpha detection provided by ORNL. The light from a scintillation in the YAP scintillator was transmitted through a fiber optic face plate to a photomultiplier tube for detection of the alpha particles. The generator weighted $30 \mathrm{lb}$ and consumed $\sim 50$ watts of power (Fig. 4.2). The time-tagged neutron beam from the generator was a fan-shaped beam produced by inserting an aperture between the photomultiplier tube (PMT) and the outside of the fiber optic face plate. The aperture was a 52-mm-wide slit in the horizontal direction and was $11 \mathrm{~mm}$ high. The electronics for alpha detection was such that $\sim 3 \%$ of the alpha triggers were false for the slit aperture. The percentage of the alphas impinging on the YAP scintillators that were counted was $\sim 85 \%$. The shape of the time-tagged neutron beam from this aperture was measured $85 \mathrm{~cm}$ from the target production in the generator (see Figs. 4.3 and 4.4). The time-tagged neutron beam has a full width at half maximum of $11^{\circ}$ in the $11-\mathrm{mm}$ direction. For this measurement, the slit was rotated $90^{\circ}$ so the measurement could be easily performed with a horizontally scanning apparatus. For the measurement of the beam profile in the long dimension, the slit was horizontally located. The time-tagged neutron beam is full width at half maximum of $\sim 40^{\circ}$ in the horizontal direction. The neutron beam is shifted because of the momentum of the accelerated ions in the accelerator (minus angle in direction of acceleration of the beam). For the measurements with the casting, the casting was centered in the time and directionally tagged neutron beam, both horizontally and vertically. The measurement of the shape of the time-tagged neutron beam is necessary for proper calculations of the measured results. 


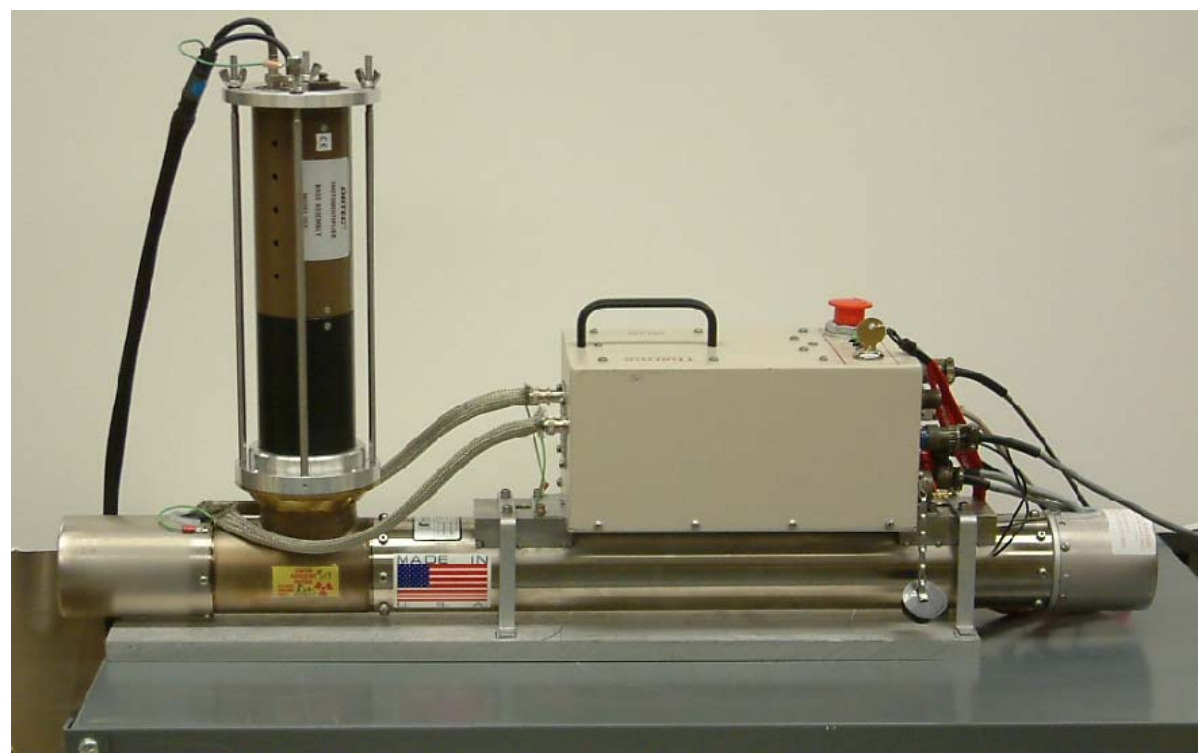

Fig. 4.2. The DT generator with an ORTEC base and PMT for alpha detection attached.

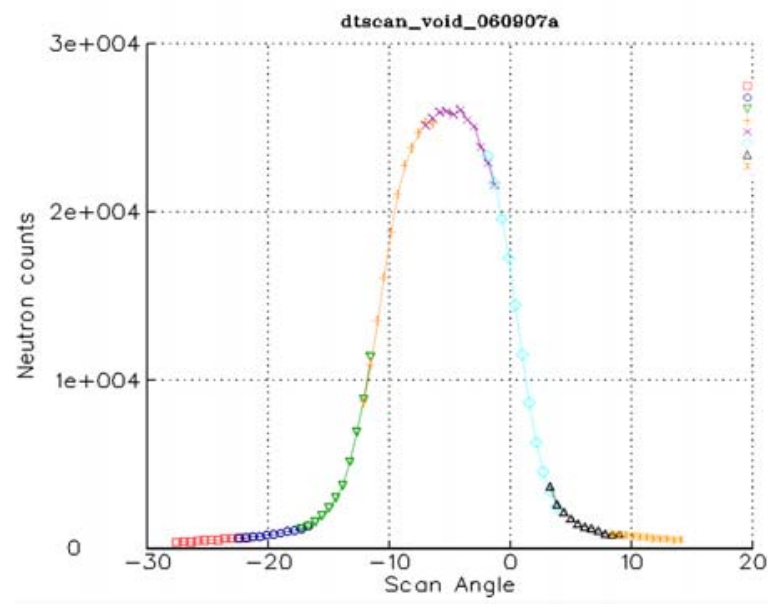

Fig. 4.3. Neutron counts as a function of angle across the slit aperture in the $11-\mathrm{mm}$ vertical direction.

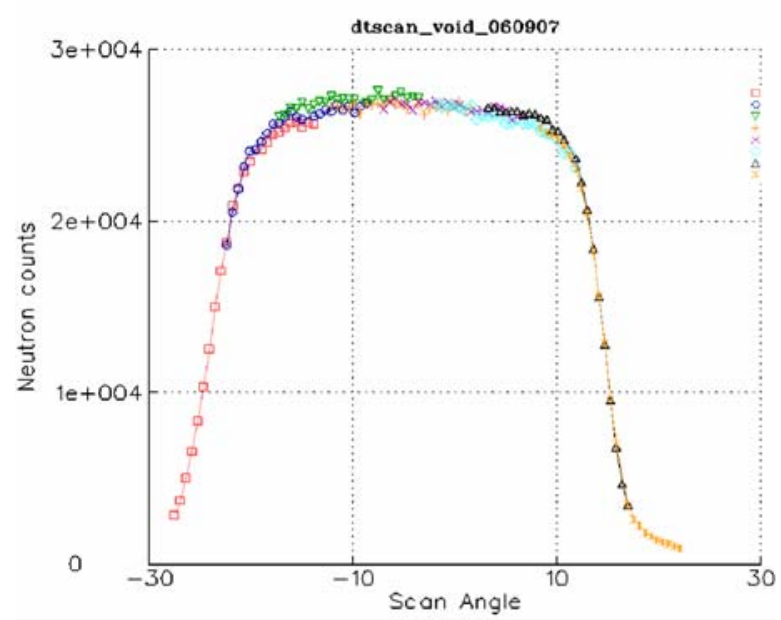

Fig. 4.4. Neutron counts as function of angle across the slit aperture in the 52-mm horizontal direction.

\subsection{RADIATION DETECTORS}

A wide variety of radiation detectors were considered for these measurements. It was decided that the smaller the detector and the closer to the castings the less the measured prompt decay would be affected by detector effects and neutron time-of-flight effects to the detectors. The neutron detectors reflect neutrons and could change the prompt decay of the fission chains if the detectors were large. The smaller the detector, the less the light propagation effects in the detector. Four $1 \times 1 \times 6$ in.-long plastic scintillators with the long dimension perpendicular to axes of the castings and adjacent to the outer 
surface of the casting cans were used in most measurements. The detectors were enclosed in 0.635 -cm.thick lead shields on four $1 \times 6$ in. surfaces and on the $1 \times 1$ in. surface. The small surface of the lead shield was adjacent to the steel table.

The detection efficiency as a function of energy was obtained from a time-of-flight measurement in air where the $\mathrm{Cf}$ source was separated $110 \mathrm{~cm}$ from the detectors, and the centers of both were $120 \mathrm{~cm}$ above the floor. These measurements were performed with the lead shields around the detectors. This information is necessary for comparison of measurement with calculations. These measurements also obtained the energy threshold for neutron detection. These measurements were performed daily before measurements with HEU and appropriate adjustments were made as needed to ensure reproducibility of the measurements. The efficiency per incident neutron can be determined from the time distribution of neutrons after $\mathrm{Cf}$ fission that gives the neutron energy, the number of detections at a given energy, the number of neutrons per $\mathrm{Cf}$ fission, the neutron energy distribution of neutrons from $\mathrm{Cf}$, the solid angle subtended by the detector, and the total number of Cf fissions. A typical time distribution of counts as a function of time after $\mathrm{Cf}$ fission is given in Fig. 4.5. The initial peak is from gamma rays that arrive at the detectors at $\sim 3.6 \mathrm{~ns}$. This is followed by the neutron peak at $50 \mathrm{~ns}$. After $100 \mathrm{~ns}$, neutrons reflected from the floor are detected.

The data from Fig 4.5 are used to obtain the detection efficiency as a function of energy and the energy threshold for detection of neutrons. Typical results are given in Fig. 4.6, and the maximum values and thresholds are summarized in Table 4.1. The efficiency increase between 1 and $2 \mathrm{MeV}$ stays constant for a small range of energies and then decreases after $3 \mathrm{MeV}$ because of the reduction of the hydrogen cross section with energy and because most if the events in the detector are neutron scattering by hydrogen. The neutron thresholds varied between 1.08 and $1.31 \mathrm{MeV}$, and the maximum detection efficiencies varied from 23 to $25 \%$ per incident neutron.

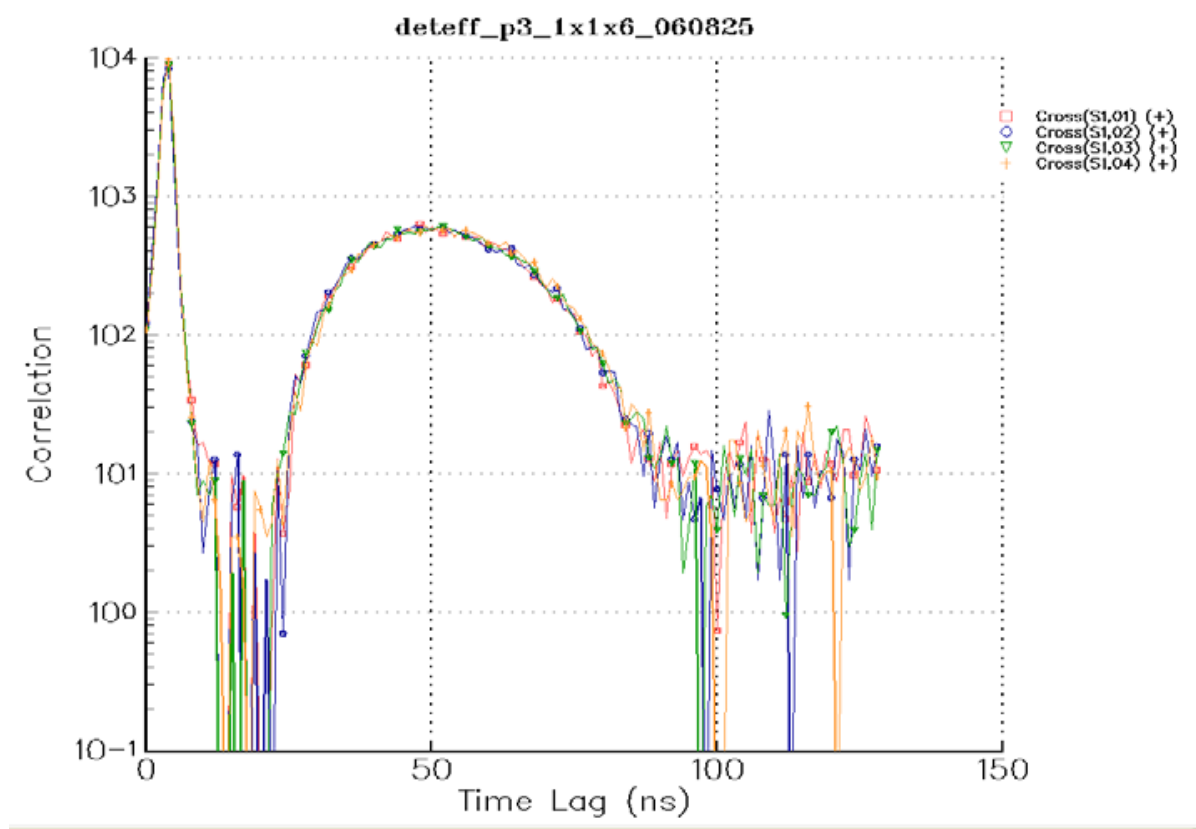

Fig. 4.5. Typical time distributions of neutrons after $\mathrm{Cf}$ fission to determine the detection efficiency as a function of energy. 


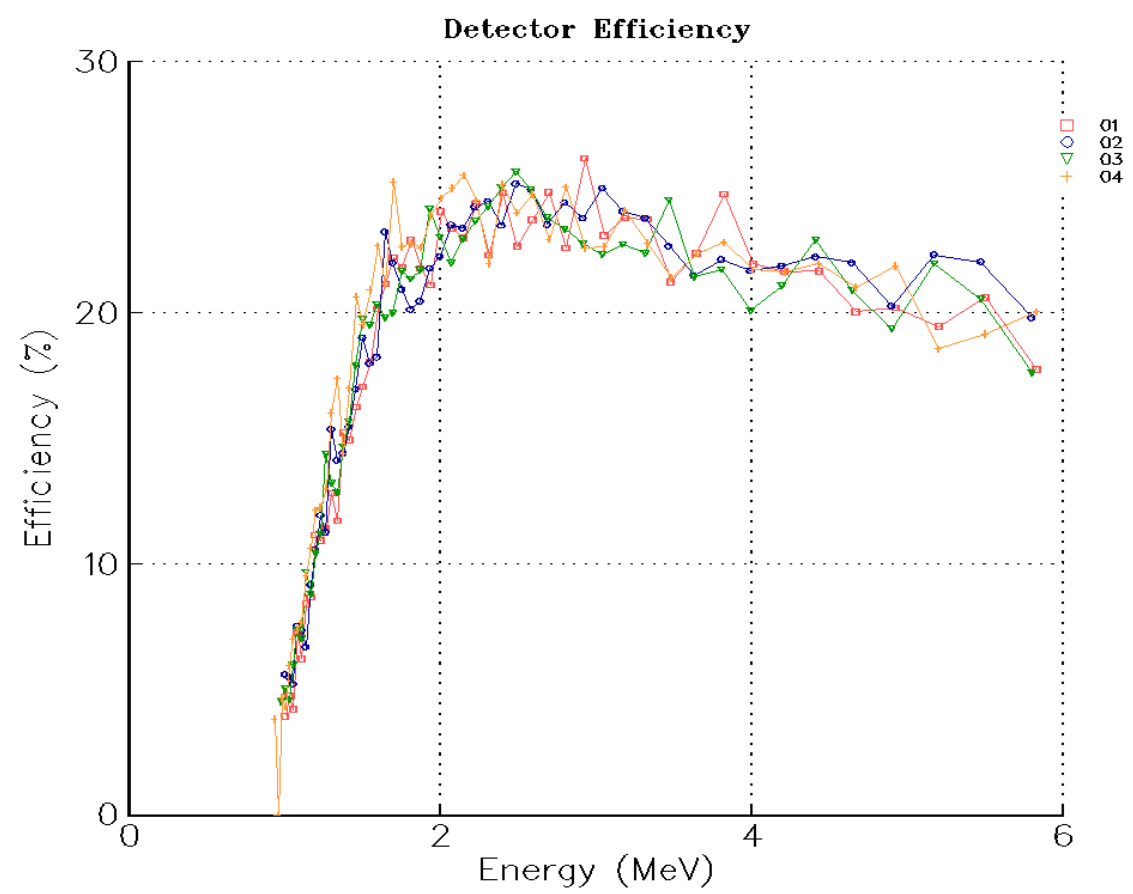

Fig. 4.6. Typical detection efficiency as a function of neutron energy.

Table 4.1. Maximum neutron detection efficiency (\%) and energy threshold (MeV) for neutron energy detection for the various days of operation

\begin{tabular}{|c|cc|cc|cc|cc|}
\hline \multirow{2}{*}{ Measurement date } & \multicolumn{6}{|c|}{ Neutron energy threshold (MeV) and maximum efficiency (\%) } \\
\cline { 2 - 8 } & \multicolumn{2}{|c|}{ Detector 1 } & \multicolumn{2}{|c|}{ Detector $\mathbf{2}$} & \multicolumn{2}{c|}{ Detector 3 } & \multicolumn{2}{c|}{ Detector 4 } \\
\hline $08 / 07 / 06$ & 1.25 & 23.7 & 1.19 & 24.5 & 1.25 & 24.4 & 1.28 & 23.0 \\
$08 / 08 / 06$ & 1.24 & 23.7 & 1.15 & 24.1 & 1.21 & 23.9 & 1.26 & 24.6 \\
$08 / 16 / 06$ & 1.22 & 24.0 & 1.21 & 24.8 & 1.22 & 24.5 & 1.25 & 24.0 \\
$08 / 17 / 06$ & 1.33 & 24.2 & 1.29 & 22.7 & 1.17 & 23.6 & 1.21 & 23.9 \\
$08 / 18 / 06$ & 1.29 & 24.2 & 1.17 & 24.2 & 1.20 & 23.8 & 1.21 & 23.4 \\
$08 / 21 / 06$ & 1.18 & 23.3 & 1.17 & 24.1 & 1.20 & 23.3 & 1.22 & 23.8 \\
$08 / 22 / 06$ & 1.21 & 23.1 & 1.17 & 24.1 & 1.13 & 23.3 & 1.22 & 24.2 \\
$08 / 23 / 06$ & 1.16 & 24.5 & 1.09 & 25.1 & 1.15 & 23.8 & 1.23 & 25.5 \\
$08 / 24 / 06$ & 1.24 & 23.4 & 1.14 & 23.3 & 1.14 & 23.0 & 1.22 & 23.6 \\
$08 / 25 / 06$ & 1.30 & 24.5 & 1.30 & 24.5 & 1.23 & 24.3 & 1.09 & 24.7 \\
$08 / 28 / 06$ & 1.17 & 23.6 & 1.14 & 23.7 & 1.19 & 23.8 & 1.18 & 24.1 \\
$08 / 29 / 06$ & 1.13 & 23.7 & 1.10 & 23.2 & 1.11 & 23.6 & 1.21 & 24.0 \\
$08 / 31 / 06$ & 1.21 & 23.7 & 1.23 & 23.9 & 1.09 & 23.6 & 1.28 & 23.9 \\
\hline
\end{tabular}

${ }^{a}$ The first number under each detector heading is neutron energy threshold in $\mathrm{MeV}$ followed by the efficiency in percent.

\subsection{NMIS PROCESSOR}

The NMIS processor is a ten-data channel, fast ( $1 \mathrm{GHz})$ time correlator that measures (1) the time distribution of events in one detector heading with respect to a previous event in the same detector (equivalent to a single detector Rossi-alpha measurement), (2) the time distribution of events in one detector with respect to a previous event in another detector (equivalent to a two-detector Rossi-alpha measurement), and (3) the time distribution of an event in a detector with respect to a time and 
directionally tagged source event in the alpha detector for the case of a DT generator or time-tagged emission from $\mathrm{Cf}$ fission in a parallel plate ionization chamber. All these time coincidence distributions are measured simultaneously. In addition, the processor measures multiplets (the number of times a detection event occurs in a time interval) in which the time interval is source triggered, the detection event is triggered, and time-coincidence distributions are randomly triggered among any three detection channels, including the source. Only five NMIS input data input channels were used in these measurements: one for the time-tagged source events and four for the detectors.

\section{PREMEASUREMENT CALCULATIONS ${ }^{2}$}

Prior to the measurements, the neutron multiplication factor and the prompt neutron time behavior after Cf fission was calculated using the Monte Carlo code MCNP-PoLiMi [4]. The calculations were performed for up to six castings although only five castings were used in the measurements because of time constraints. The casting configurations were close packed. The $\mathrm{k}_{\mathrm{eff}}$ values for the bare annular castings with no nearby reflector material are given in Table 5.1.

Table 5.1. $\mathrm{K}_{\text {eff }}$ values for the bare castings

\begin{tabular}{cc}
\hline Number & $\mathbf{K}_{\text {eff }}$ \\
\hline 1 & 0.4535 \\
2 & 0.5518 \\
3 & 0.6426 \\
4 & 0.6916 \\
5 & 0.7180 \\
6 & 0.7403 \\
\hline
\end{tabular}

The calculated time distribution of fission after initial $\mathrm{Cf}$ fission for a central $\mathrm{Cf}$ source and no reflector materials present is given in Fig. 5.1. This is similar to a measurement for an internal fission detector that was the HEU itself. This internal fission system decay will be attempted to be measured with an external detector and reflection from nearby materials and the room by correlating the detector response with timetagged Cf fission. For the maximum number of castings, the time decay is reduced four decades in $300 \mathrm{~ns}$, while that for one casting is reduced four decades in $\sim 150 \mathrm{~ns}$.

The calculated time distribution of fission after a previous fission in the system (which simulates a twodetector Rossi alpha measurement that detects all fission) is given in Fig. 5.2. This distribution differs significantly from that of Fig. 5.1 only in the amplitude and the initial time behavior. These distributions represent the time decay fission chains inside the system. The distributions of Fig. 5.2 represent the ideal Rossi alpha measurement where the detector is the fission material itself.

2 The calculations and the comparisons of calculations with measurements were the work of James Henkel of G2 Corporation. 


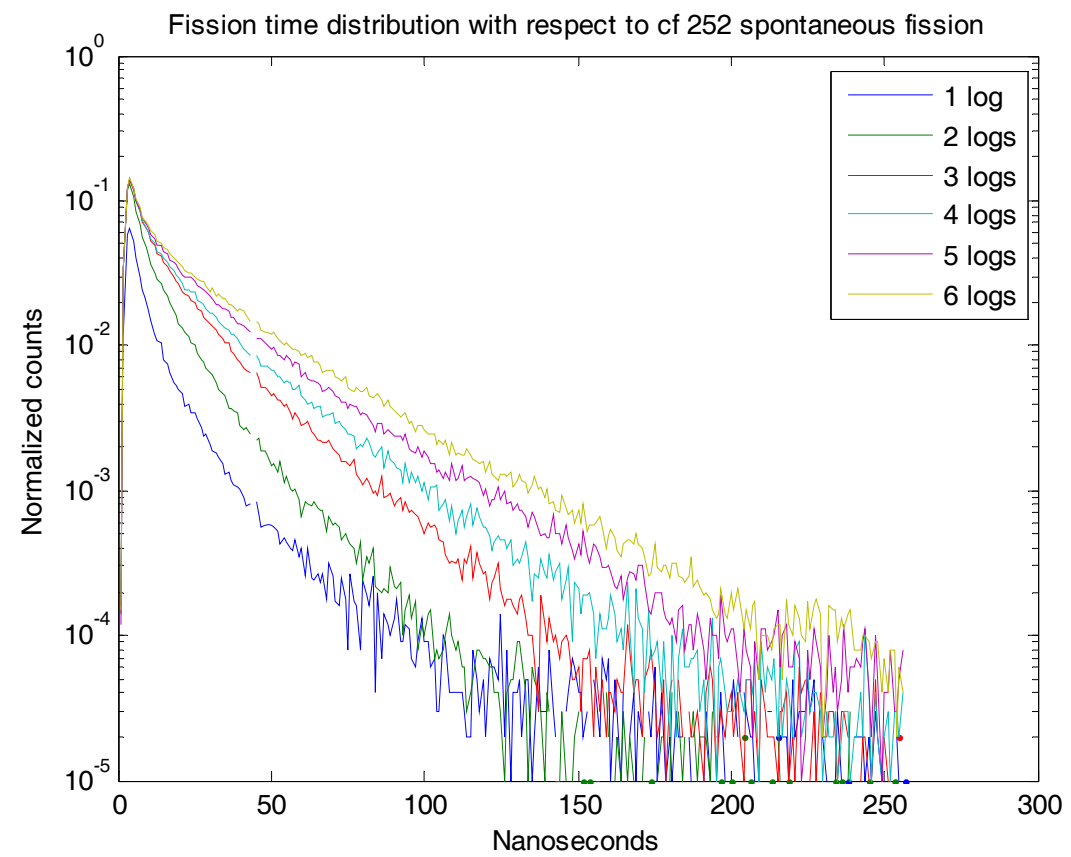

Fig. 5.1. Calculated time distribution of fission as a function of time after Cf fission with the source located in the center of a casting.

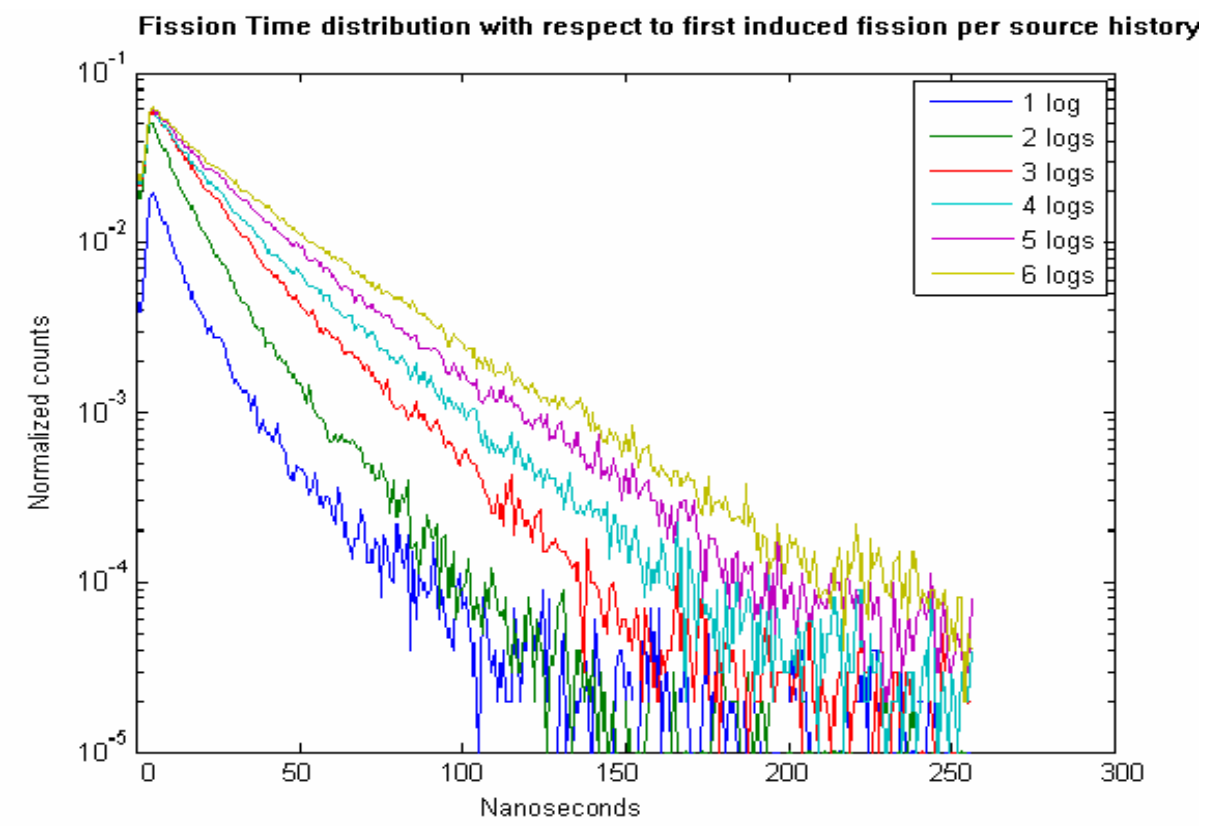

Fig. 5.2. Time distribution of fission after a previous fission as a function of the number of castings. 


\section{ROOM RETURN MEASUREMENTS}

Three measurements were performed without HEU castings on the measurements table, and the detectors located on the measurements table were in locations typical of a measurement performed with HEU. The first measurement was with the DT generator with a 1.5-in.-diam. aperture with the generator $740 \mathrm{~cm}$ from the north wall in the direction of the tagged beam. The DT generator was $533 \mathrm{~cm}$ from the east wall, $503 \mathrm{~cm}$ from the west wall, $326 \mathrm{~cm}$ from the south wall, and the room was $12.8 \mathrm{~m}$ high. The other two measurements were with $\mathrm{Cf}$ located as in the measurements but with the measurements table 40 in. above the floor and also $29 \mathrm{in}$. above the floor. In all measurements with the Cf source, it was $3.25 \mathrm{in}$. above the steel table. The DT generator results are given in Fig. 6. 1. Note that the background subtracted level is about $10^{-7}$. In this measurement the generator target spot was 3 in. above the table. Neutrons with energy of $14.1 \mathrm{MeV}$ would scatter back from the floor in less than $100 \mathrm{~ns}$. The time distribution out to $1000 \mathrm{~ns}$ is from $14.1 \mathrm{MeV}$ neutrons scattering between the walls, floor, and ceiling of the room until they slow down below the detection threshold of $\sim 1.2 \mathrm{MeV}$.

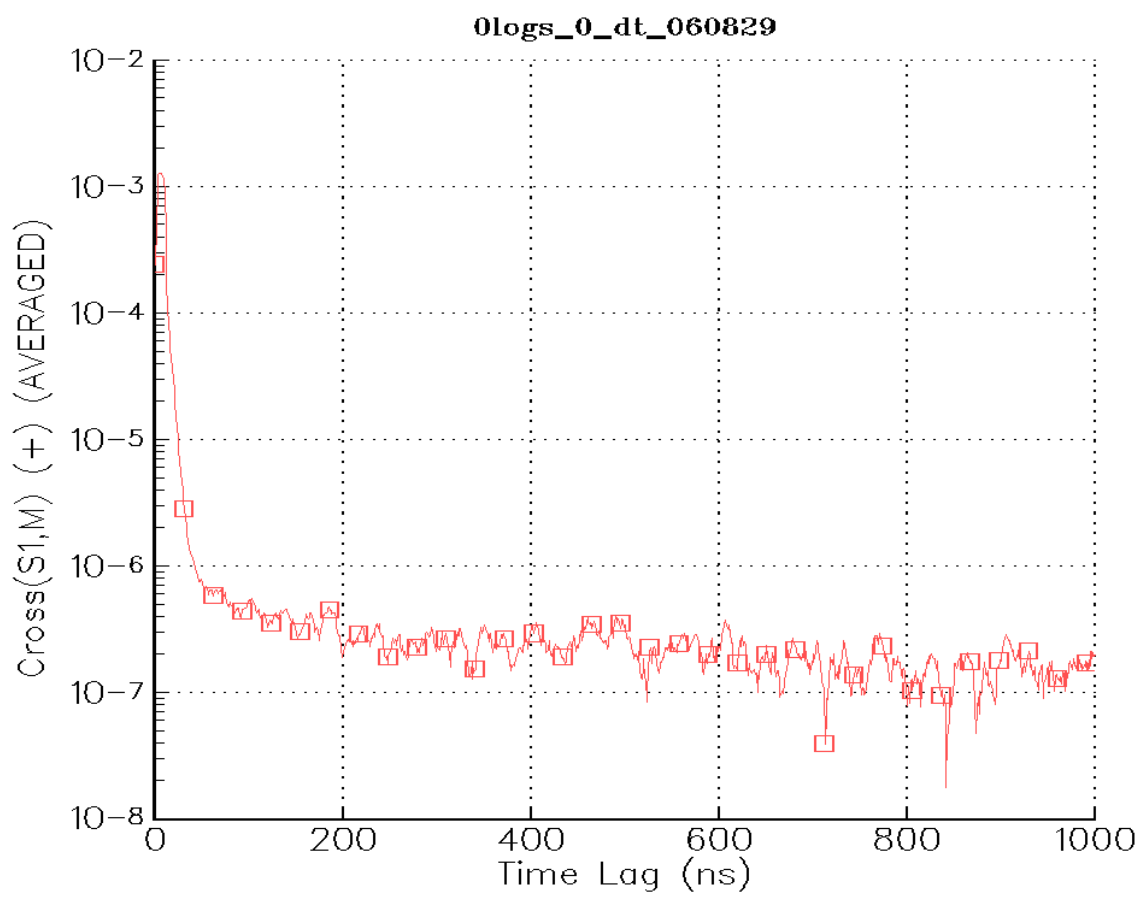

Fig. 6.1. Pulsing an empty room with the DT generator.

A similar result for the $\mathrm{Cf}$ source with the table top $\sim 40$ in. above the floor is given in Fig. 6.2. Note that the level after $300 \mathrm{~ns}$ is below $10^{-7}$ and seems to have an exponential decay, which is associated with the slowing down from scattering around the room. Part of the distribution at $\sim 120 \mathrm{~ns}$ is scattering from the floor, which can also be seen in the data of Fig. 4.4 and which is plotted out to longer times in Fig. 6.3. The time constant of the decay in the room after $200 \mathrm{~ns}$ is $\sim 11$ inverse $\mu$ s (i.e., the decrease is a factor of 2.7 in $11 \mu \mathrm{s})$.

The neutron detection threshold is $\sim 1.2 \mathrm{MeV}$, and late neutrons from the walls have less chance to be detected than reflected DT neutrons because some of them are slowed down below the detection threshold in less time. The measurements with the table lowered $11 \mathrm{in}$. are compared with those at $\sim 40 \mathrm{in}$. in Fig. 6.5. The increase at $\sim 100 \mathrm{~ns}$ for the measurement at $29 \mathrm{in}$. is caused by additional neutrons from the floor reflection. These results indicate the time at which the effects of room return affect the measurements and cannot be used to correct the measurements with the castings present. They do not 
represent the interaction of the room return with the HEU of the castings.

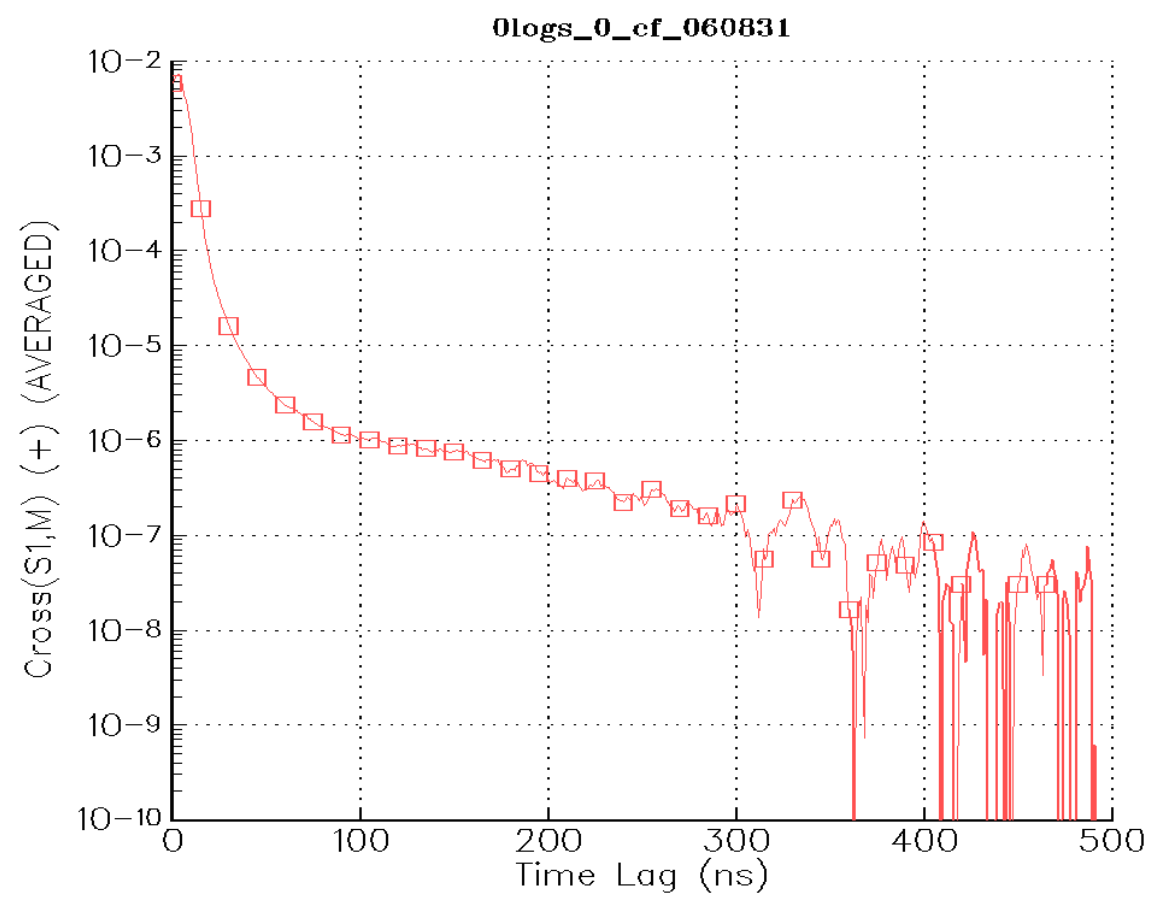

Fig. 6.2. Room return from $\mathrm{Cf}$ with table top at 40 in. from the floor.

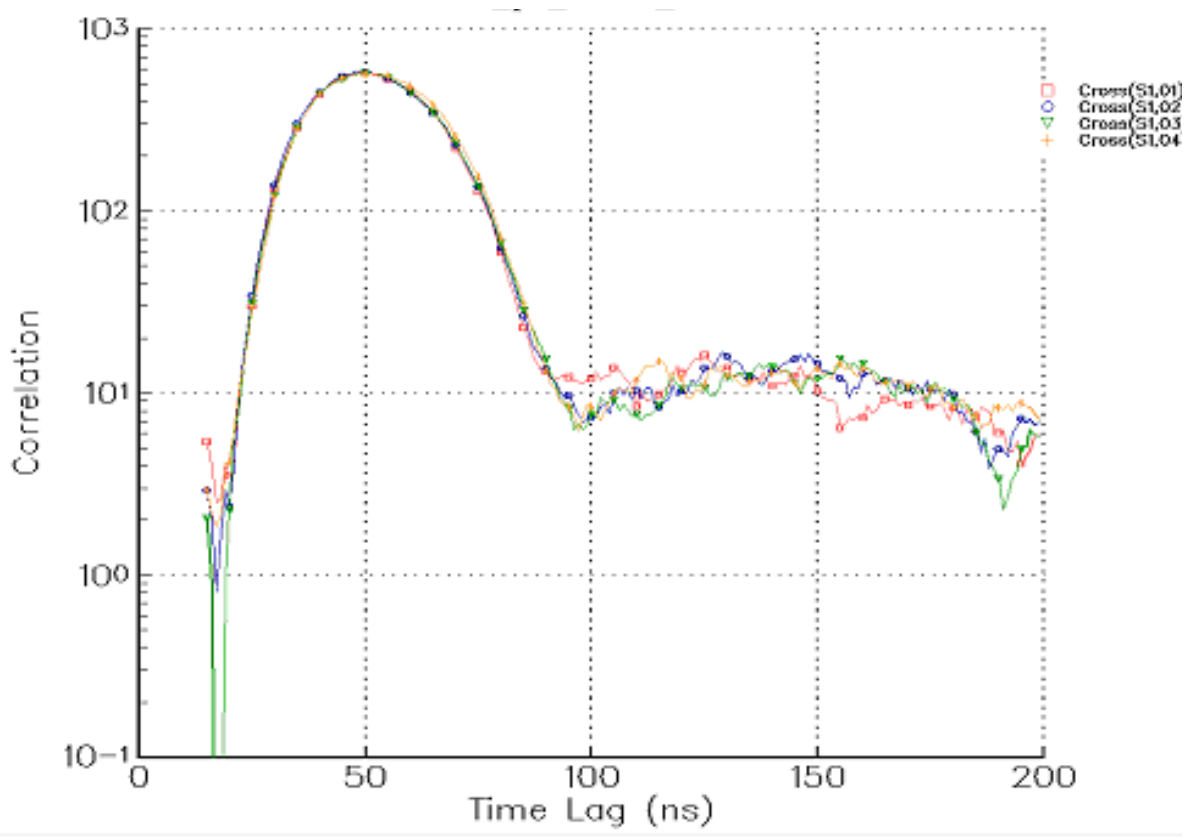

Fig. 6.3. Time distribution for all four detectors from Fig. 4.4 averaged over nine channels and for longer times showing floor-reflected neutrons from 100 to 200 ns. 


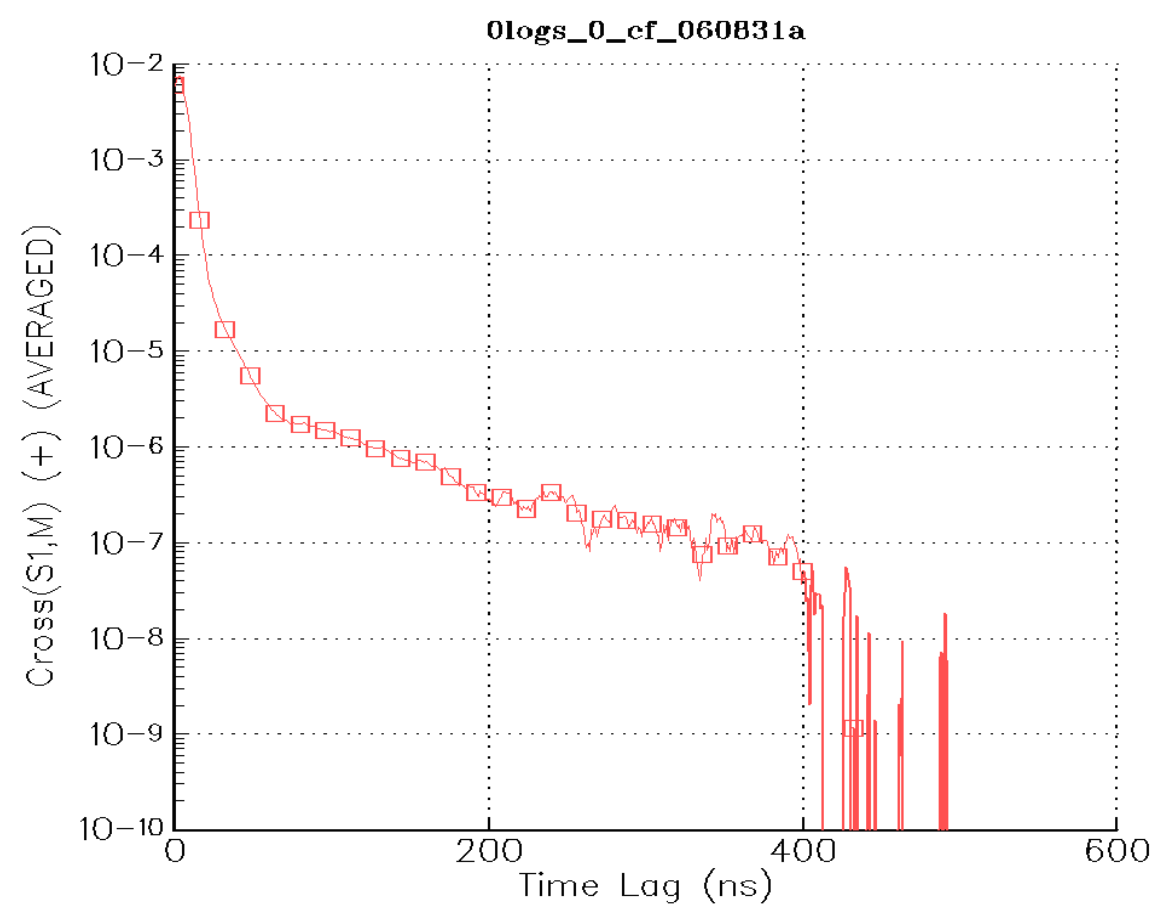

Fig. 6.4. Cf source and no castings with the table 29 in. above floor.

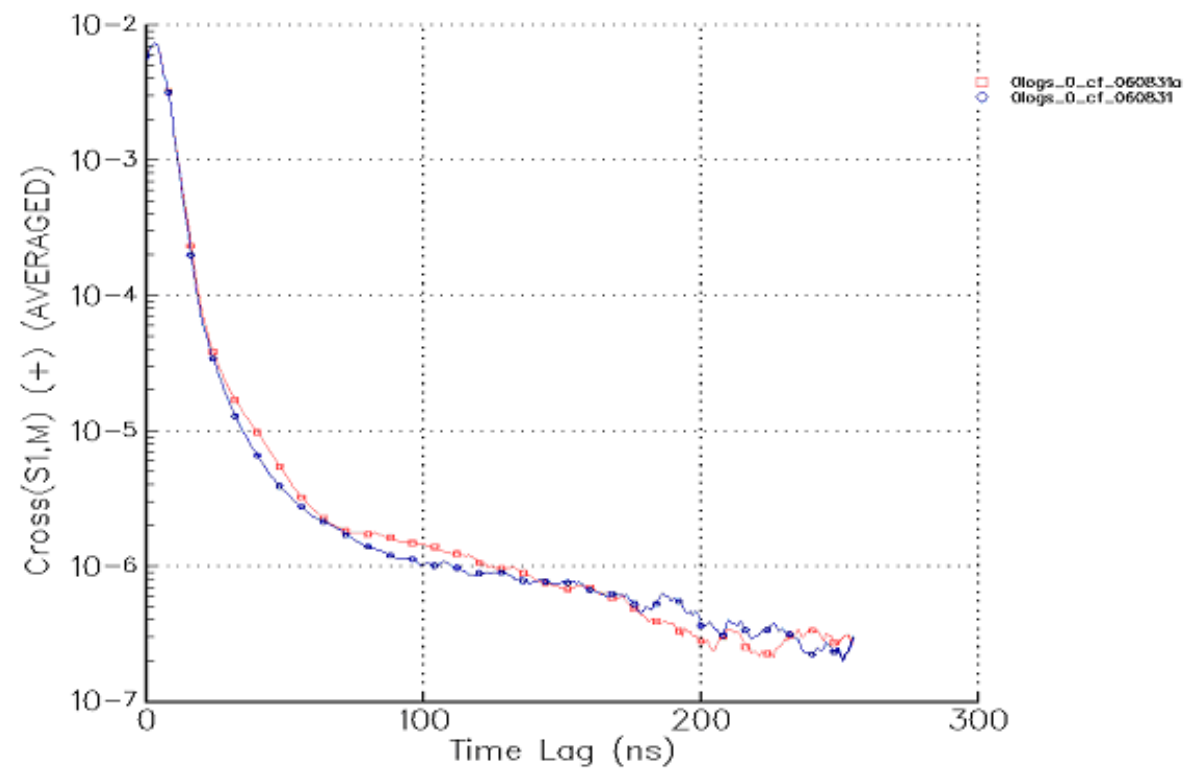

Fig. 6.5. Comparison of the measurements with $\mathrm{Cf}$ and no castings with the measurements table 29 and $40 \mathrm{in}$. above the floor. 


\subsection{MEASUREMENTS WITH ONE CASTING}

Measurements for one casting with the three source-detector-casting configurations are listed in detail in Appendix C.

\subsubsection{Configuration 1}

A photograph of one of the $\mathrm{Cf}$ source-detector-casting measurement configurations (configuration number 1 of Appendix C) with the detectors adjacent to the radial surface of the steel can is given in Fig. 6.6. The DT generator on the left is spaced away from the configuration to minimize neutron reflection. The $\mathrm{Cf}$ ionization chamber amplifier, which is held in position by a clamp, located the $\mathrm{Cf}$ source on the axis of the casting in the annular can. The time distributions of counts after $\mathrm{Cf}$ fission are shown in Fig. 6.7 and are essentially identical for all four detectors except for small differences near the time lag of $5 \mathrm{~ns}$. The very early part of the time distribution is dominated by directly transmitted neutrons from the $\mathrm{Cf}$ source. After this time, the fission chain multiplication process determines the time response and the response from all detectors is the same. This results from the detection efficiencies being nearly the same for all detectors and their symmetric location with respect to the source and casting. This measurement is equivalent to a pulsed neutron measurement with a randomly pulsed neutron source. The time distribution is not exponential and is independent of the detector location after the initial transients associated with detector location. The time distribution of coincidences between all detectors with the $\mathrm{Cf}$ source on the axis is shown in Fig. 6.8. Except for small differences near time lag zero, the time dependence is the same for all detector combinations. This measurement is a two-detector Rossi-alpha measurement that measures the time distribution of counts in one detector with respect to a previous count in another detector.

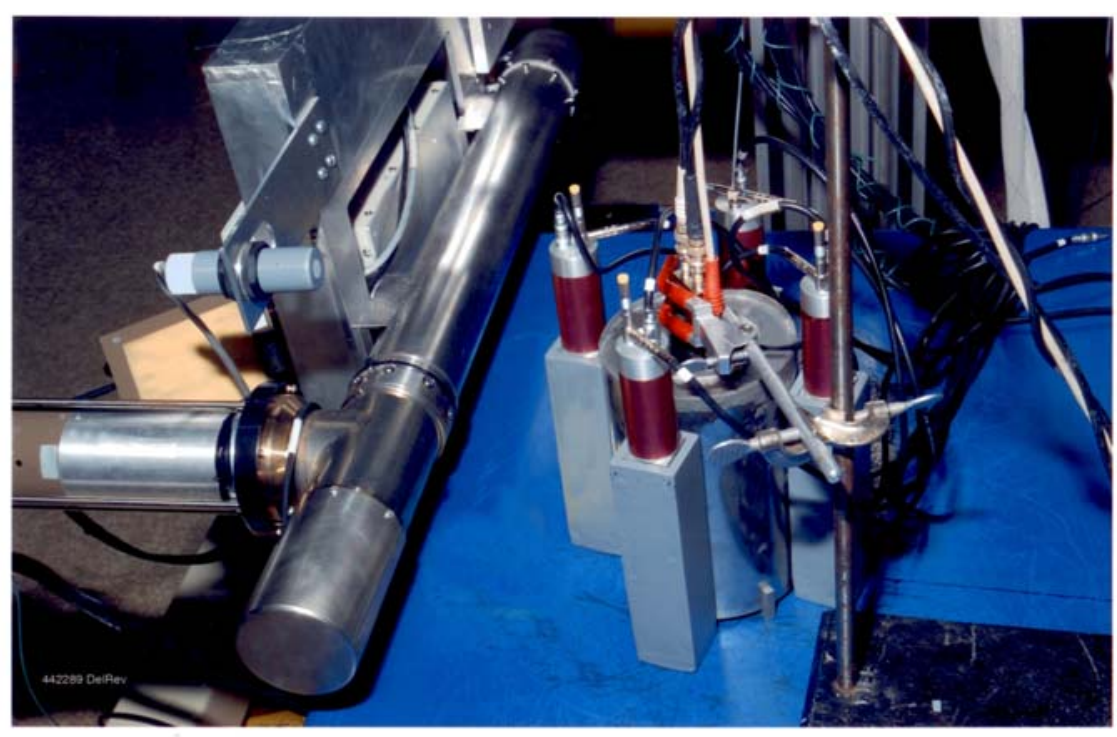

Fig. 6.6. The Cf source-detector-single uranium metal casting with the $\mathbf{C f}$ source on the axis of the casting. The generator is not appropriately positioned for a measurement with the generator as a source and was turned off for measurements with Cf. 


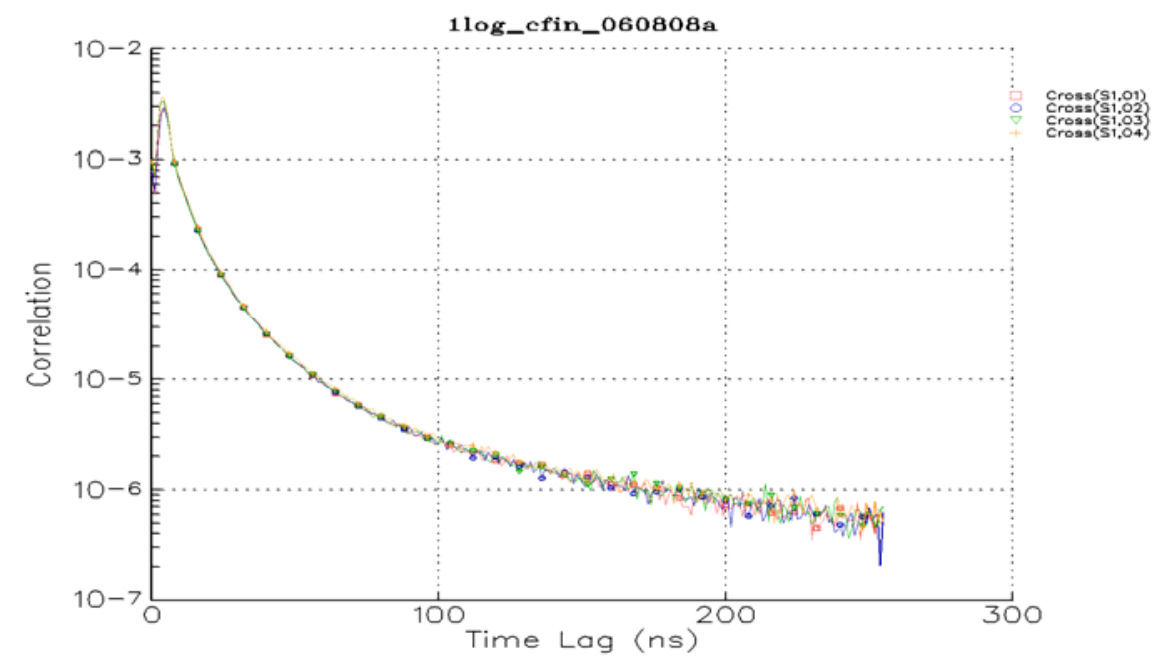

Fig. 6.7. Time distribution of coincidence count per $\mathrm{Cf}$ fission between each of the four detectors and the $\mathrm{Cf}$ source fission with the $\mathrm{Cf}$ source on the axis of the annular casting.

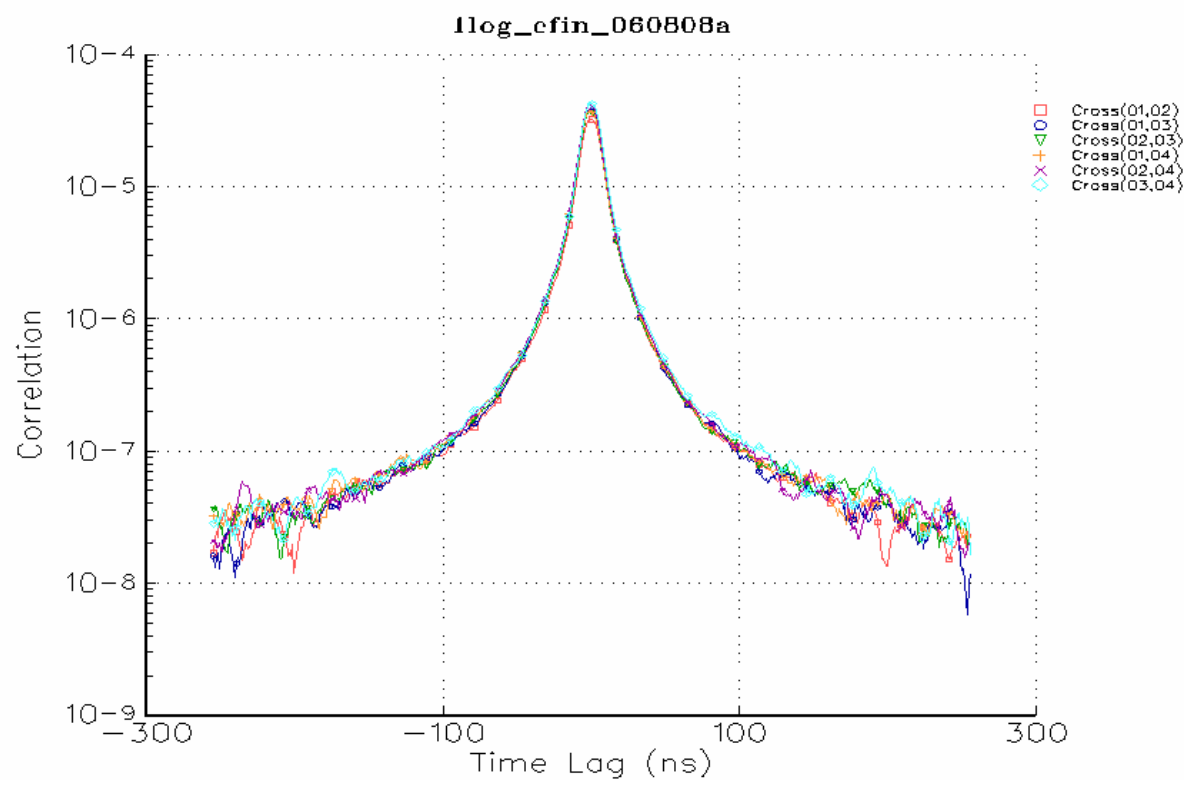

Fig. 6.8. Time distribution of coincidences between detectors with the $\mathrm{Cf}$ source internal. The data on this plot are averaged over five time bins of $1 \mathrm{~ns}$ each.

\subsubsection{Configuration 2}

The time distributions of counts in each of three detectors after $\mathrm{Cf}$ fission with the $\mathrm{Cf}$ source external for configuration 2 are shown in Fig. 6.9 and are essentially identical for all detectors except for small differences near a time lag of $15 \mathrm{~ns}$. This configuration has the $\mathrm{Cf}$ source in contact with the casting can and three detectors $90^{\circ}$ apart in contact with the surface of the steel can with the casting. The initial transmission from $\mathrm{Cf}$ for detector 2 is slightly delayed more than that for detectors 1 and 3 because detector 2 is farther from the source. 


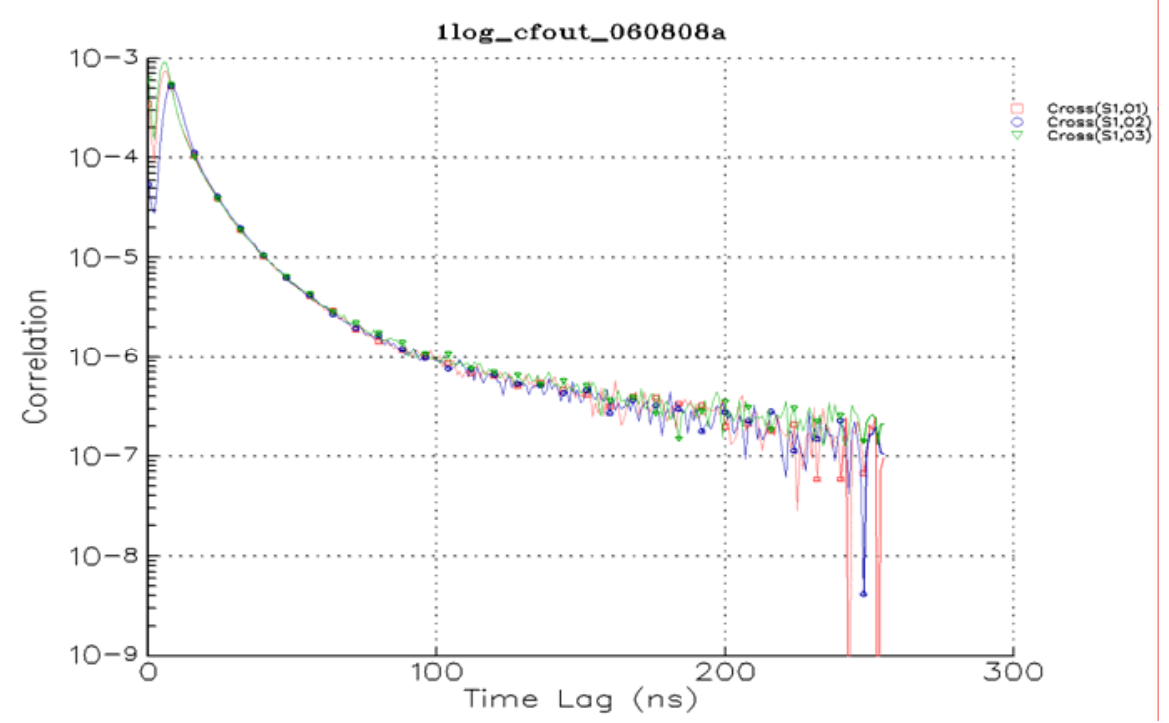

Fig. 6.9. Time distribution of coincidence count per $\mathrm{Cf}$ fission between each of the three detectors and the $\mathrm{Cf}$ source fission with an external source.

The time distribution of coincidences between all detectors with the $\mathrm{Cf}$ source external is shown in Fig. 6.10. The time distributions are slightly different: the distribution for detector 1-3 is slightly wider because the detectors are farther apart than the detector combinations 1-2 and 2-3.

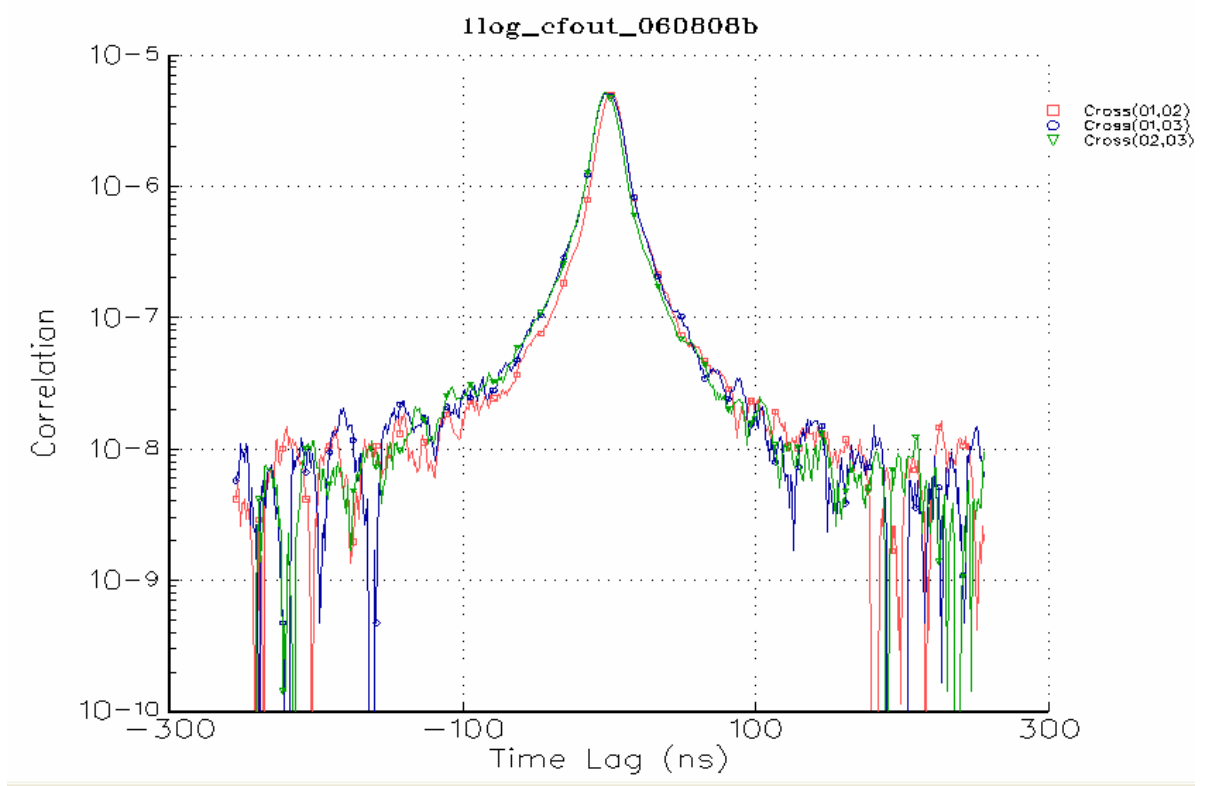

Fig. 6.10. Time distribution of coincidences between three detectors with an external Cf source.

\subsubsection{Configuration 3}

The time distributions of coincidences between the three detectors and the alpha detection in the DT generator are given in Fig. 6.11. The measurement time for the measurements with the DT generator was 
$35 \mathrm{~min}$., with an alpha detection rate of $340,000 / \mathrm{s}$. A portion of detector 2 is in the time and directionally tagged neutron beam from the DT generator and, thus, has a higher initial detection rate (up to $\sim 5 \mathrm{~ns}$ ) than the other two detectors, which are at right angles to the source. After the initial source-induced fission, fission chain multiplication emits neutron and gamma rays isotropically, and all three detectors have the same time response after $25 \mathrm{~ns}$. This indicates that the fission chain decay time measured is independent of the detector position. The observed time decay of the fission chains is not exponential as expected because of the low neutron multiplication factor.

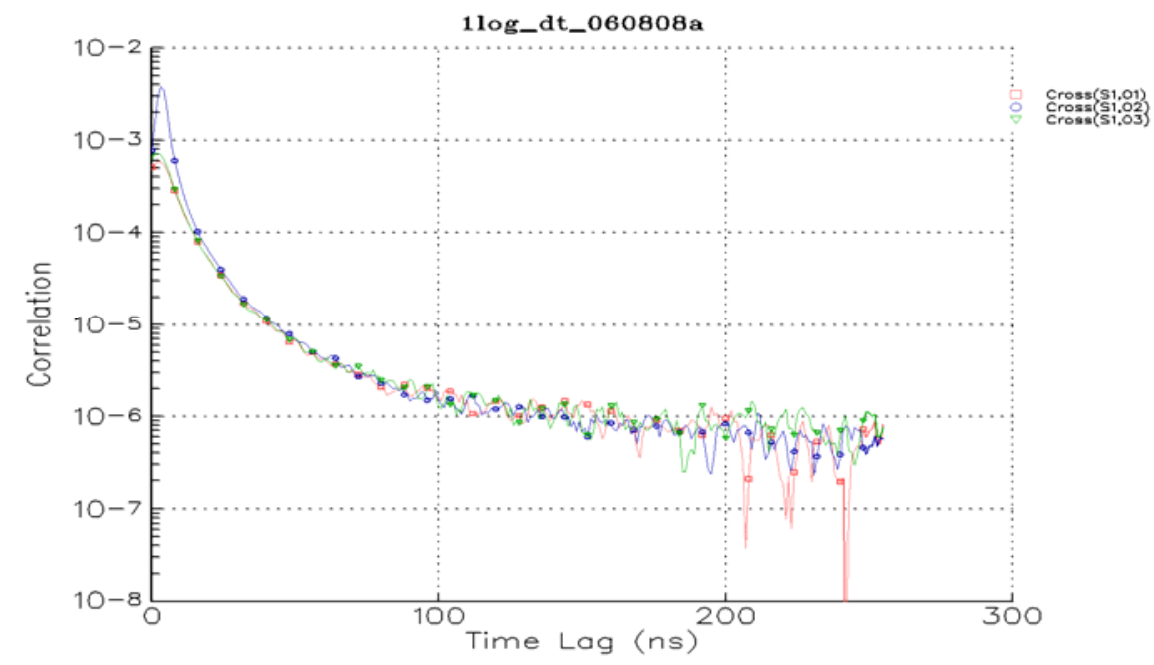

Fig. 6.11. Time distribution of coincidence count per tagged-source neutron between each of the three detectors and the DT generator neutrons with the source external.

The time distribution of coincidences between detectors 1 and 3,1 and 2, and 2 and 3 are shown in Fig. 6.12. The different widths of the time distributions are a result of the detectors' being different distances from each other: two pairs (1-2, 2-3) with adjacent detectors and one pair (1-3) with detectors on opposite sides of the castings. Except for these small differences, the time dependencies are the same. 


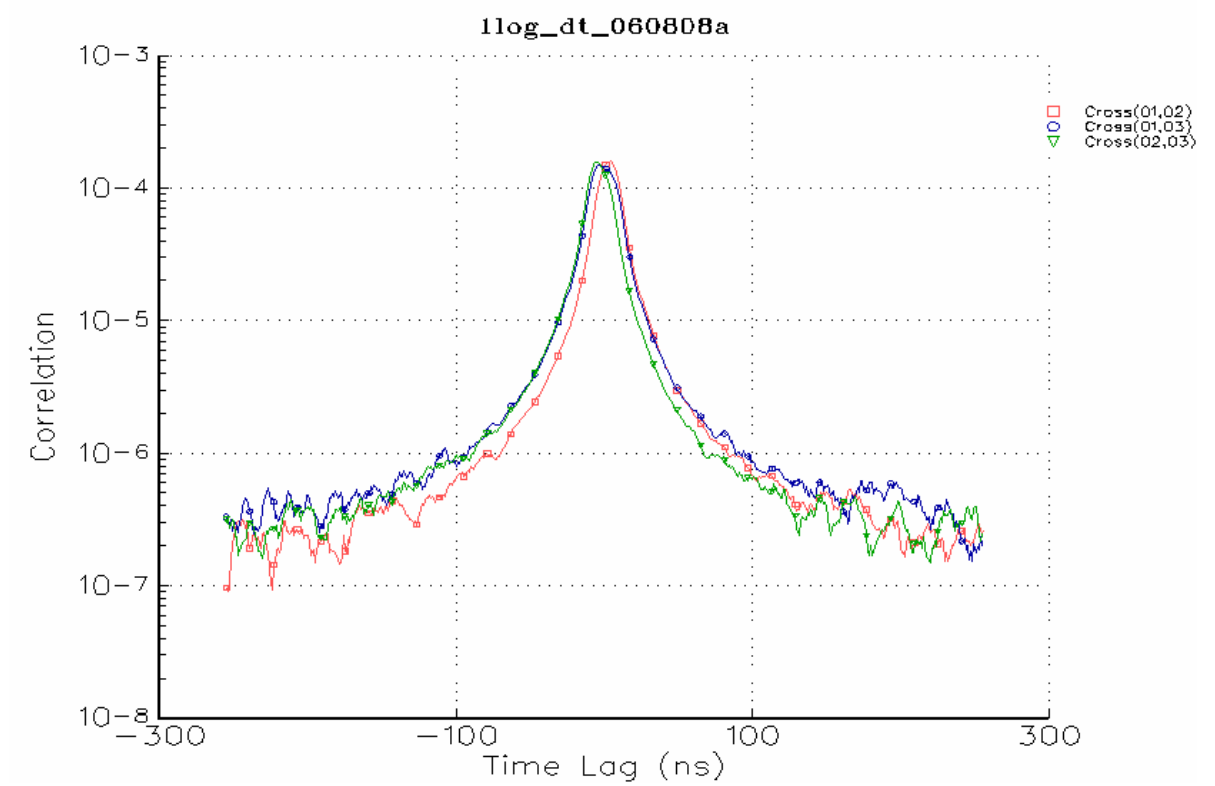

Fig. 6.12. Time distribution of coincidences between detectors for measurements with an external DT source. The data have been averaged, and all averages are not plotted.

\subsubsection{Comparison of Measurements with the Two Sources}

Figure 6.13 shows the time distributions of counts in one of the detectors after alpha detection in the DT generator, after $\mathrm{Cf}$ source fission with the $\mathrm{Cf}$ adjacent to the outside of the casting can and on the axis of the casting. The time distribution of coincidences with respect to the source persists longer for the DT generator because the 14.1-MeV neutrons bounce around the room above the detection threshold longer than they do for the fission energy neutrons from the Cf source. The room-return effects with the DT generator appear earlier because the $14.1-\mathrm{MeV}$ neutrons travel faster to the walls and floor of the room and can scatter back sooner. The time distribution of coincidences between detectors 1 and 3 for the three measurements is shown in Fig. 6.14. The time distribution of coincidences between detectors persists longer at long times because of the time and directionally tagged neutrons that pass through the assemblies scattering off the floor and walls of the room. 


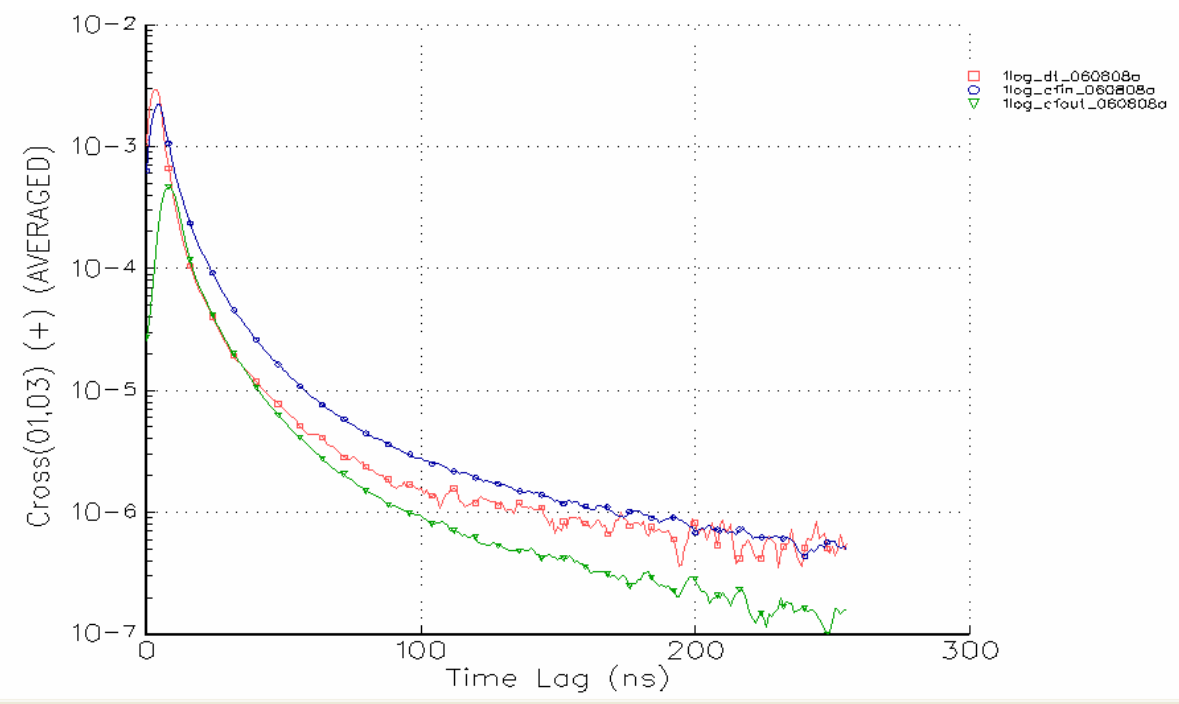

Fig. 6.13. Comparison of the time distribution of counts in one detector (2) with respect to the source event for the three measurement configurations.

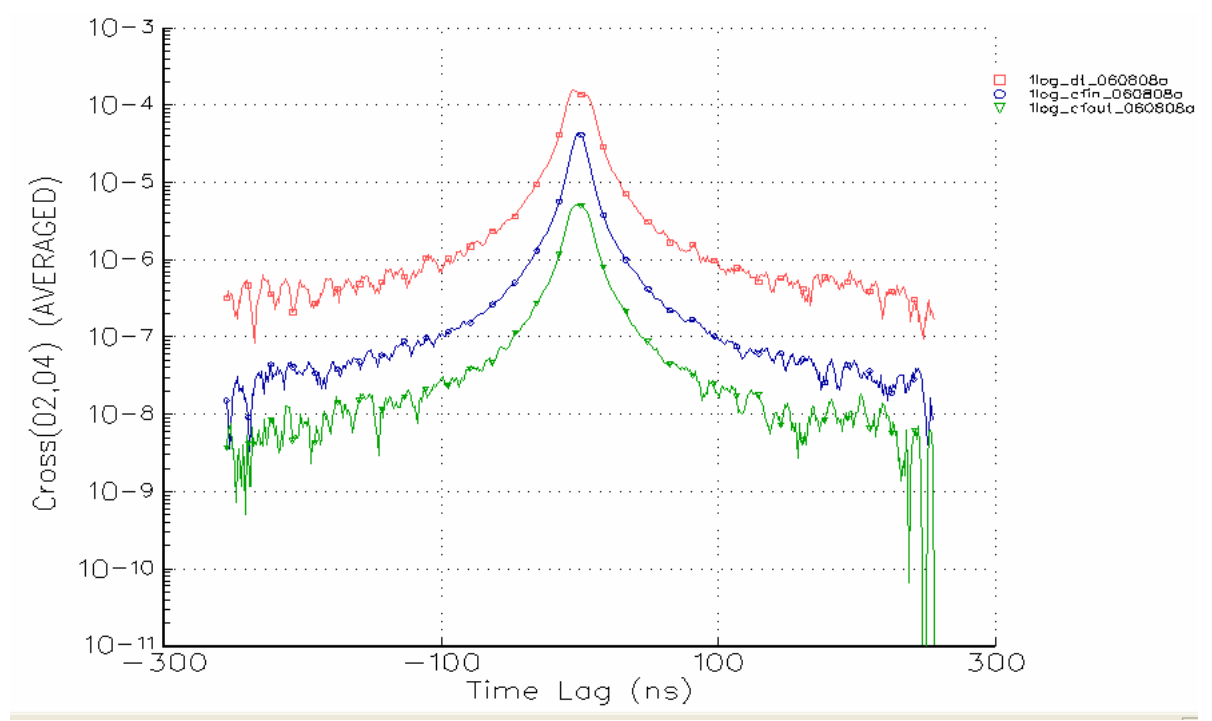

Fig. 6.14. The time distribution of coincidences between detectors on opposite sides of the casting for the three configurations.

The measured room-return effects are less for the $\mathrm{Cf}$ source. If the $\mathrm{Cf}$ source is some distance from the $\mathrm{HEU}$, the reverse is true. When the source cannot be located adjacent to the fissile material, significant numbers of neutrons from the $\mathrm{Cf}$ source scatter from the walls and floor of the room before they reach the fissile material because the time-tagged neutrons go in all directions from the source. Thus, when the $\mathrm{Cf}$ source is closely coupled to the HEU, the effects of the experimental room are reduced but not eliminated. The DT generator has advantages when spaced from the HEU because the time-tagged neutrons are directional. Thus, the use of a DT generator may not be necessary for these types of measurements as long as the $\mathrm{Cf}$ source can be in or in contact with the fissile material. 


\subsection{MEASUREMENTS WITH TWO CASTINGS}

Measurements for two castings with a wide variety of source-detector-casting configuration are listed in detail in Appendix C. A photograph of one of the $\mathrm{Cf}$ source-detector-casting measurement configurations (configuration number 4 of Appendix $\mathrm{C}$ ) with the detectors adjacent to the radial surface of the steel cans is given in Fig. 6.15. The DT generator on the left is spaced away from the configuration to minimize neutron reflection. The $\mathrm{Cf}$ ionization chamber amplifier, held in position by a clamp, located the $\mathrm{Cf}$ source on the axis of the casting in the annular can.

The time distribution of counts in each of the detectors after Cf fission is shown in Fig. 6.16. The very early transient part of these time distributions are from neutrons directly from the source, and the later time distribution is the result of the time distribution of the induced fission chain multiplication process. After the initial transients, the time die-away of the counts has the same time response. The detector adjacent to the casting with the CF source internal have a higher correlated count rate because they are closer to the source, and more fissions are occurring in the casting with the source longer time of flight of $\mathrm{Cf}$ source fission neutrons to these detectors. The time distributions of counts in one detector with respect to a previous count in another detector are given in Fig. 6.17. The data for the correlation between detectors 1 and 3 are higher than that between detectors 2 and 4 because they are adjacent to the casting with the $\mathrm{Cf}$ source internal. The time dependence is not exponential because the system is far subcritical.

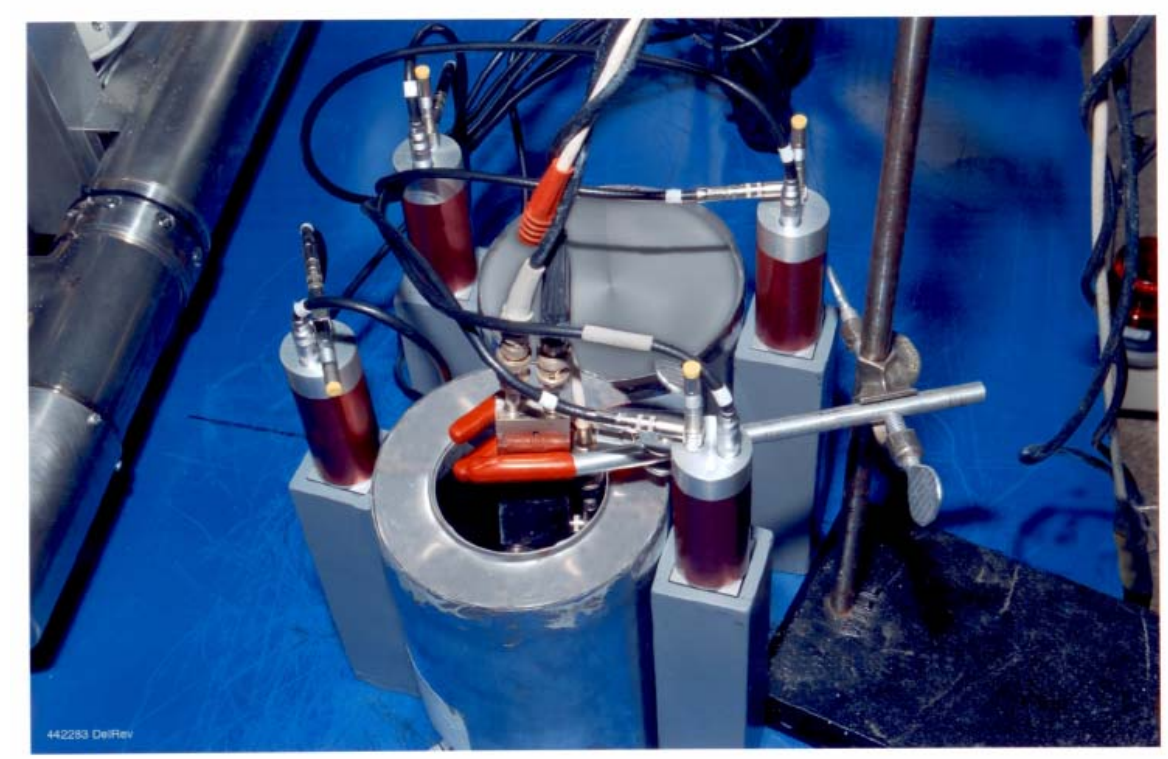

Fig. 6.15. Photograph of $\mathrm{Cf}$ source-detector-casting configuration with the Cf source internal for a measurement with two castings. 


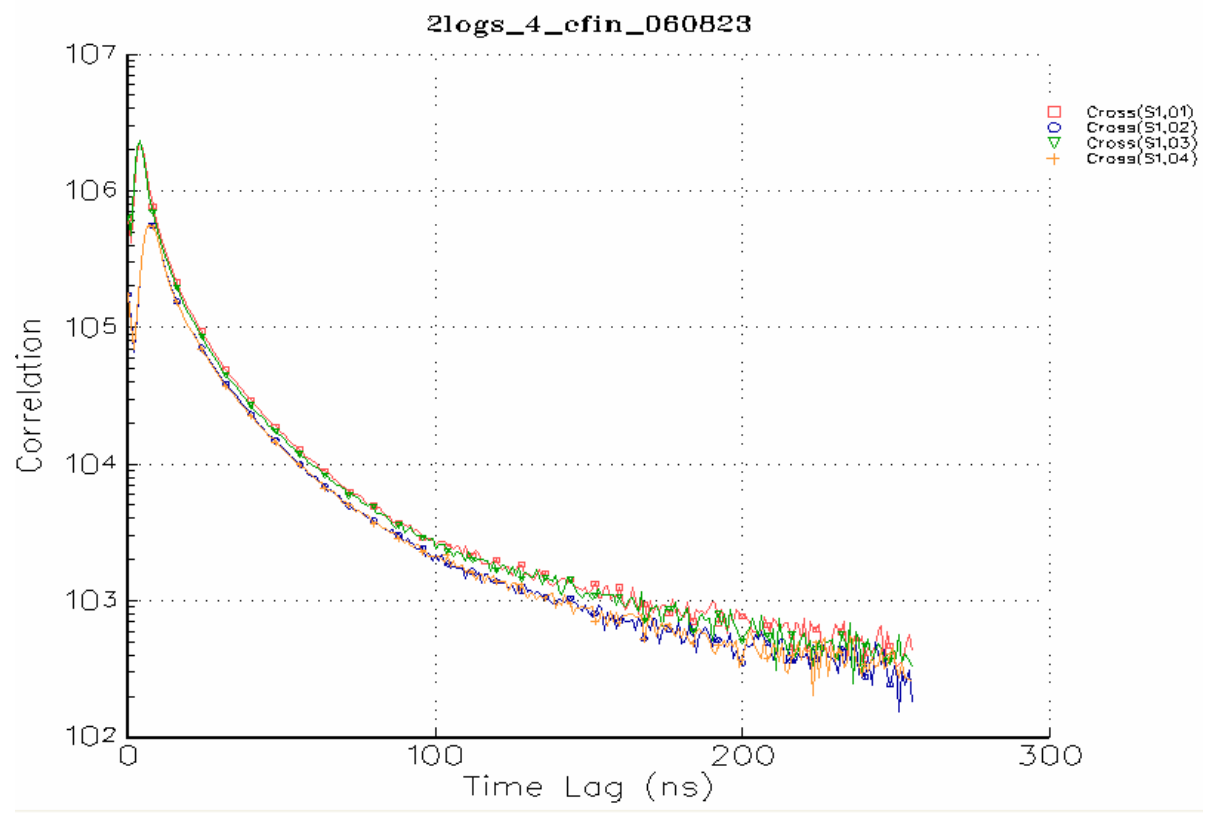

Fig. 6.16. The time distribution of counts in each of the detectors after $\mathrm{Cf}$ fission for configuration four of two castings with the $\mathrm{Cf}$ source internal.

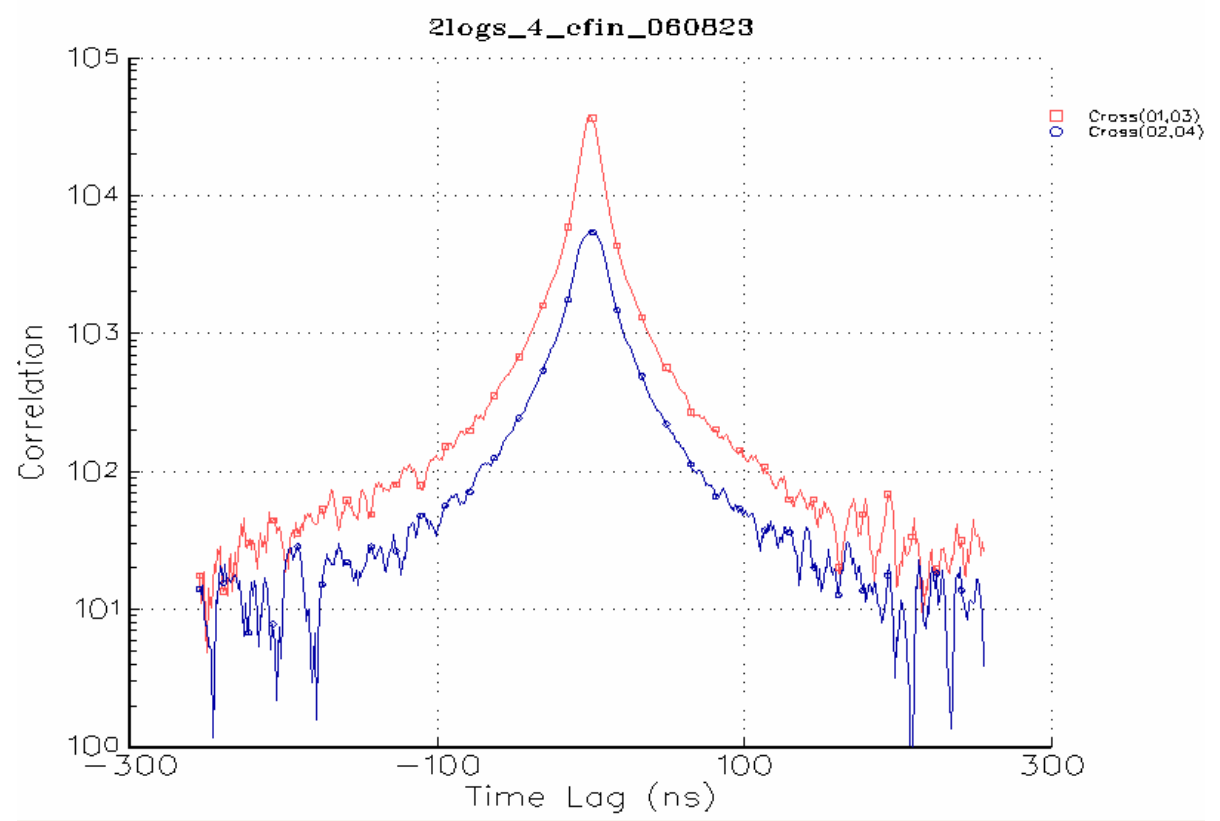

Fig. 6.17. Time distribution of counts in one detector with respect to a previous count in another detector for configuration 4 of two castings with the Cf source internal to one. 


\subsection{MEASUREMENTS WITH THREE CASTINGS}

Measurements for three castings with a wide variety of source-detector-casting configuration are listed in detail in Appendix C. The time distribution of detector counts after $\mathrm{Cf}$ fission and the time distribution of counts in a detector after a count in a previous detector are given in Figs. 6.18 and 6.19 for configuration 12 (Appendix C). Detector 1 is adjacent to the casting with the source and thus has the highest initial count rate, and detectors 2 and 4 are farthest from the source. Since detector 2 is closest to the center of the fission distribution, its response is the largest. The asymmetries in the source detector casting configuration result in time correlation between detectors not centered at time lag 0 .

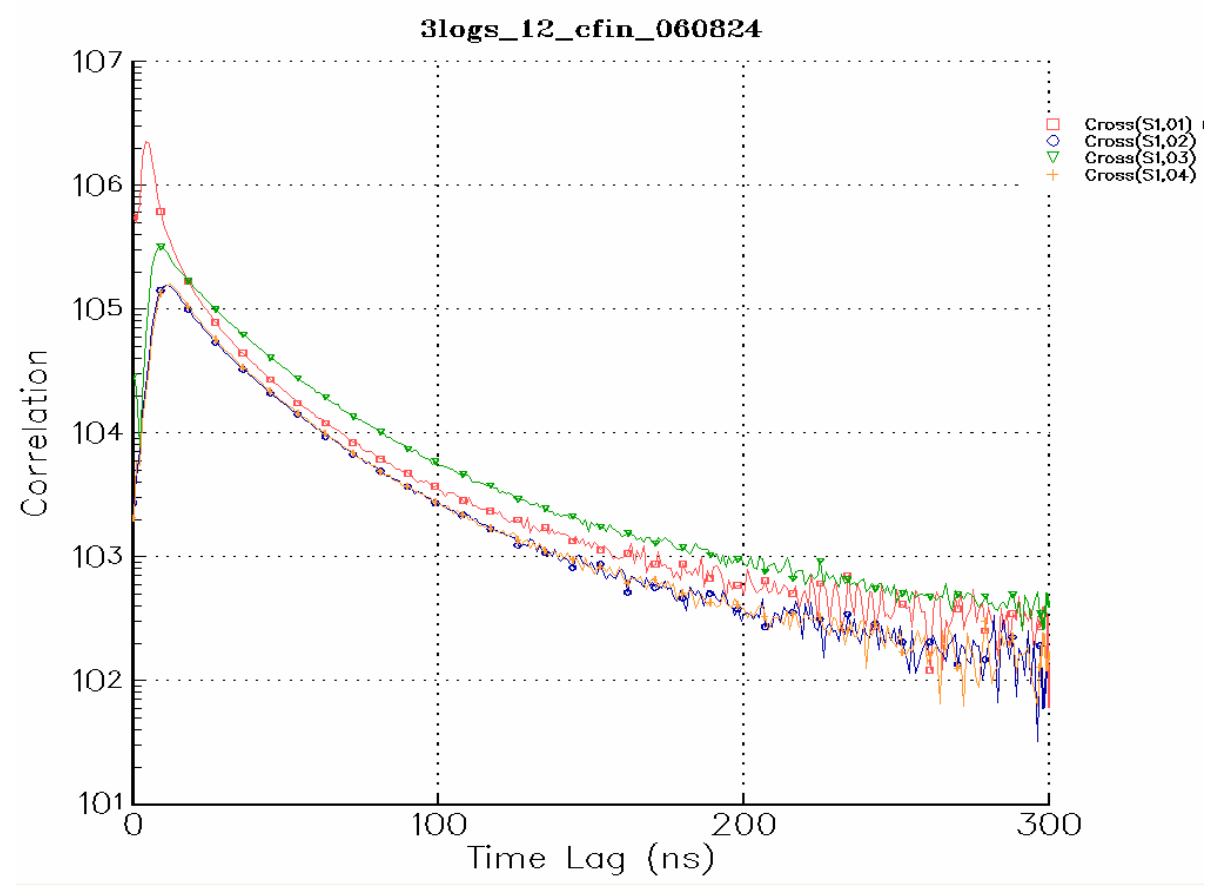

Fig. 6.18. Time distribution of count in the detectors after $\mathrm{Cf}$ fission for configuration 12 (three castings with Cf internal). 


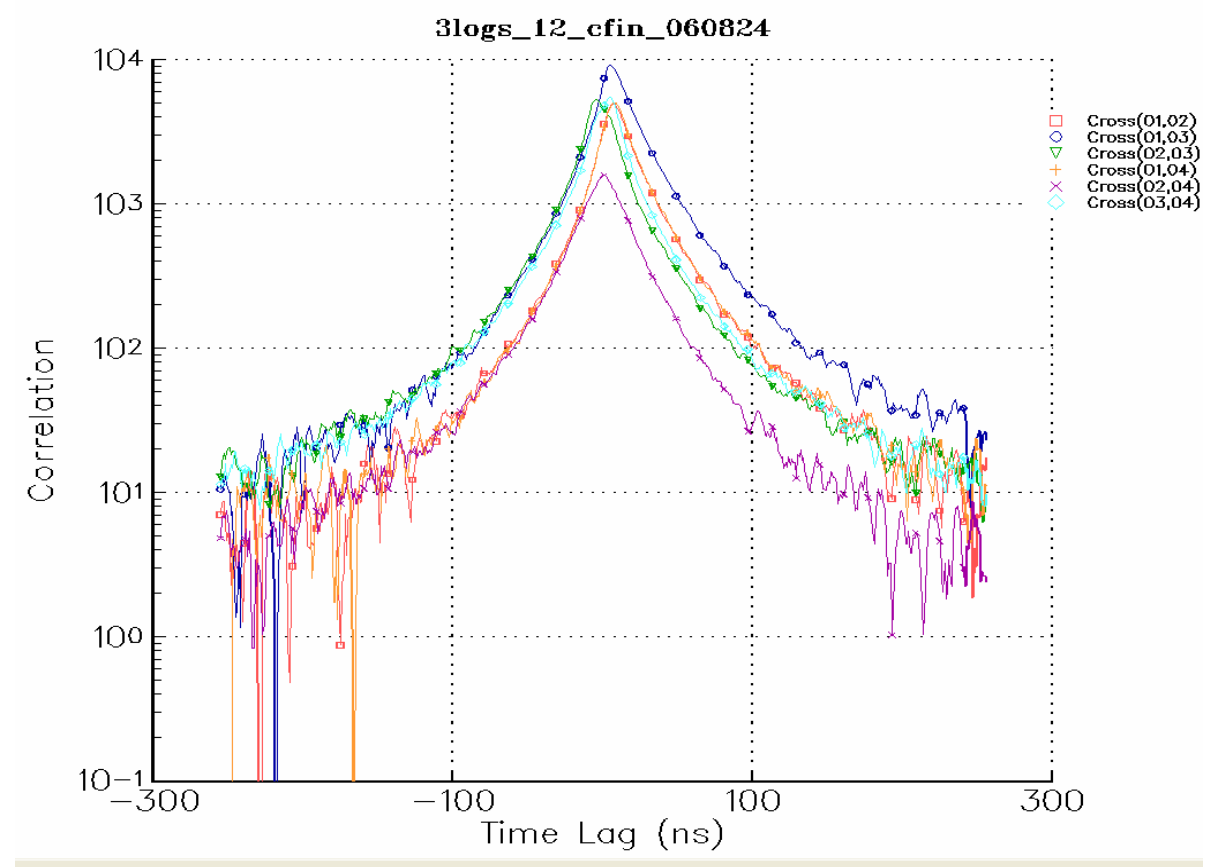

Fig. 6.19. Time distribution of counts in one detector with respect to a previous count in another detector.

\subsection{MEASUREMENTS WITH FOUR CASTINGS}

Measurements with a wide variety of source-detector-casting configuration are listed in detail in Appendix C. A photograph of one of the DT generator-detector-casting measurement configurations (configuration number 24a of Appendix C) is given in Fig. 6.20. The DT generator is touching the casting can closest to it, so the target spot where neutrons are produced is 1.5 in. away from the casting can. A line drawn through the center of the casting near the generator and the casting in the annular can is $4.3^{\circ}$ downstream from perpendicular. The detectors are located as close to the point where the castings touch as possible. 


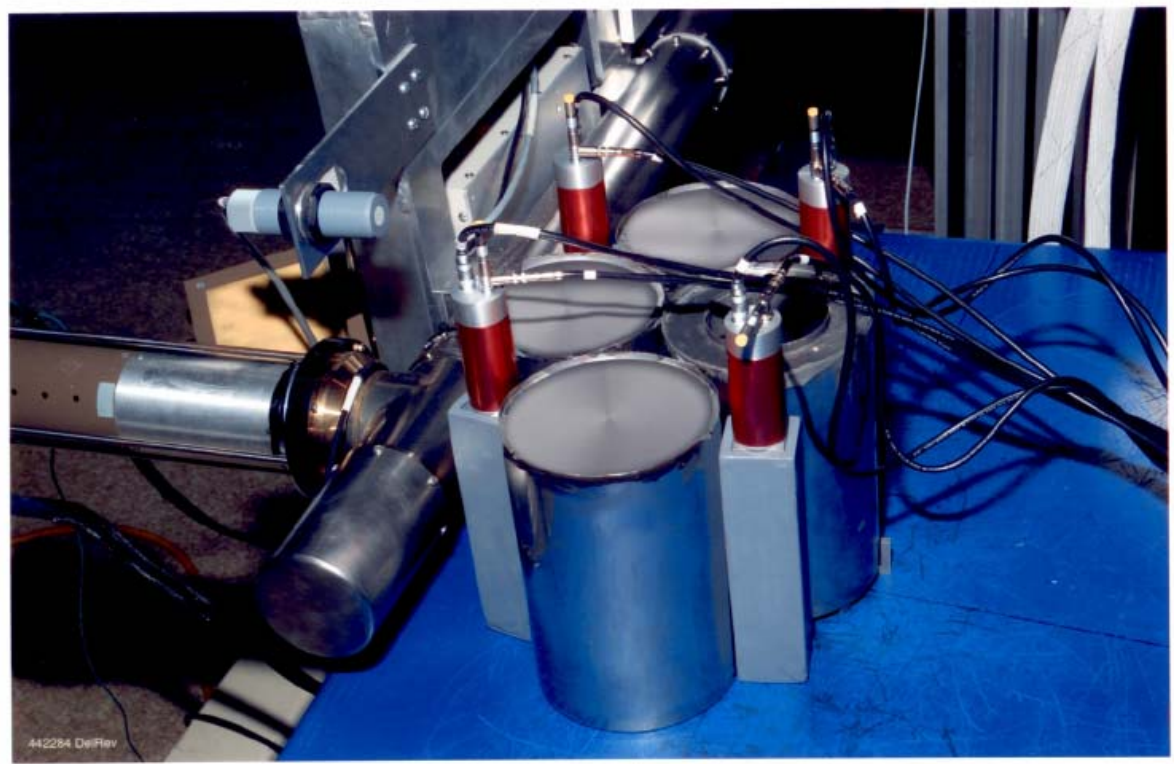

Fig. 6.20. Photograph of the DT source-detector-casting configuration 20 for measurement with four castings.

The time distribution of counts in each of the detectors after Cf fission for configuration 20 of Appendix $\mathrm{C}$ is shown in Fig. 6.21. The time distribution of detector counts after a count in a previous detector is given in Fig. 6.22 for configuration 24a.

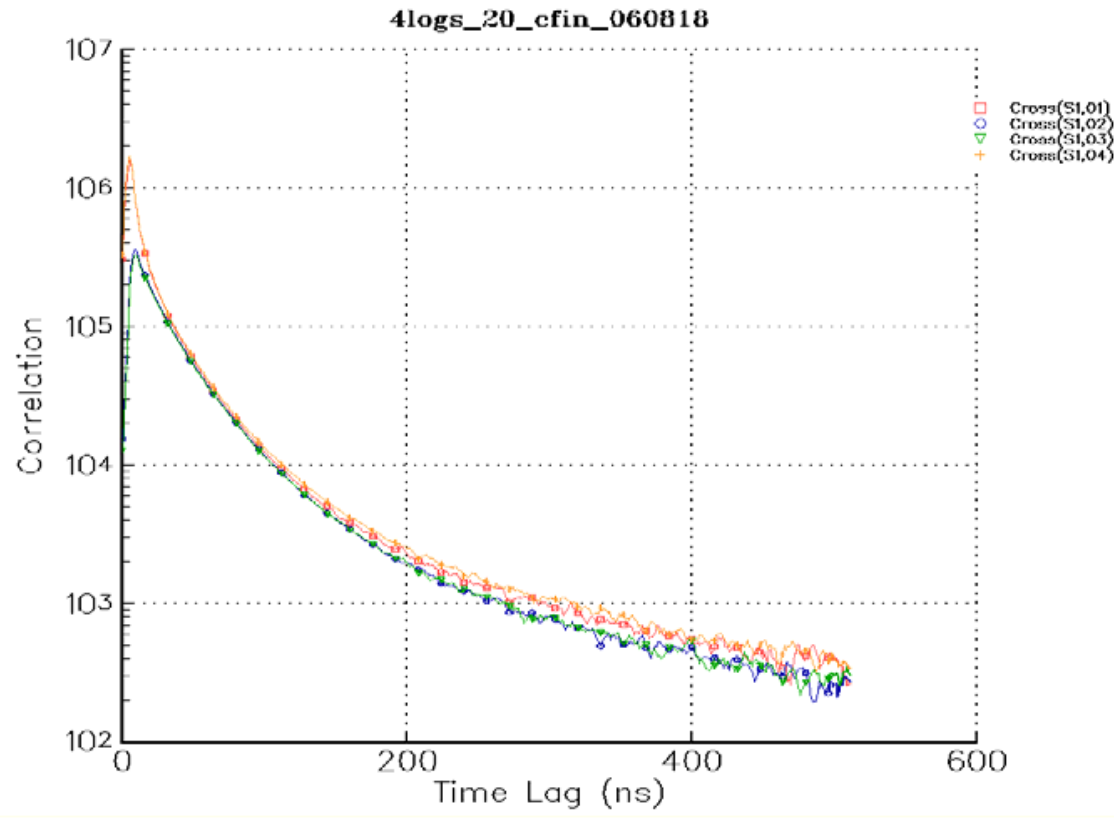

Fig. 6.21. Time distribution of count in the detectors after $\mathrm{Cf}$ fission for configuration 20 (four castings with $\mathrm{Cf}$ internal). 


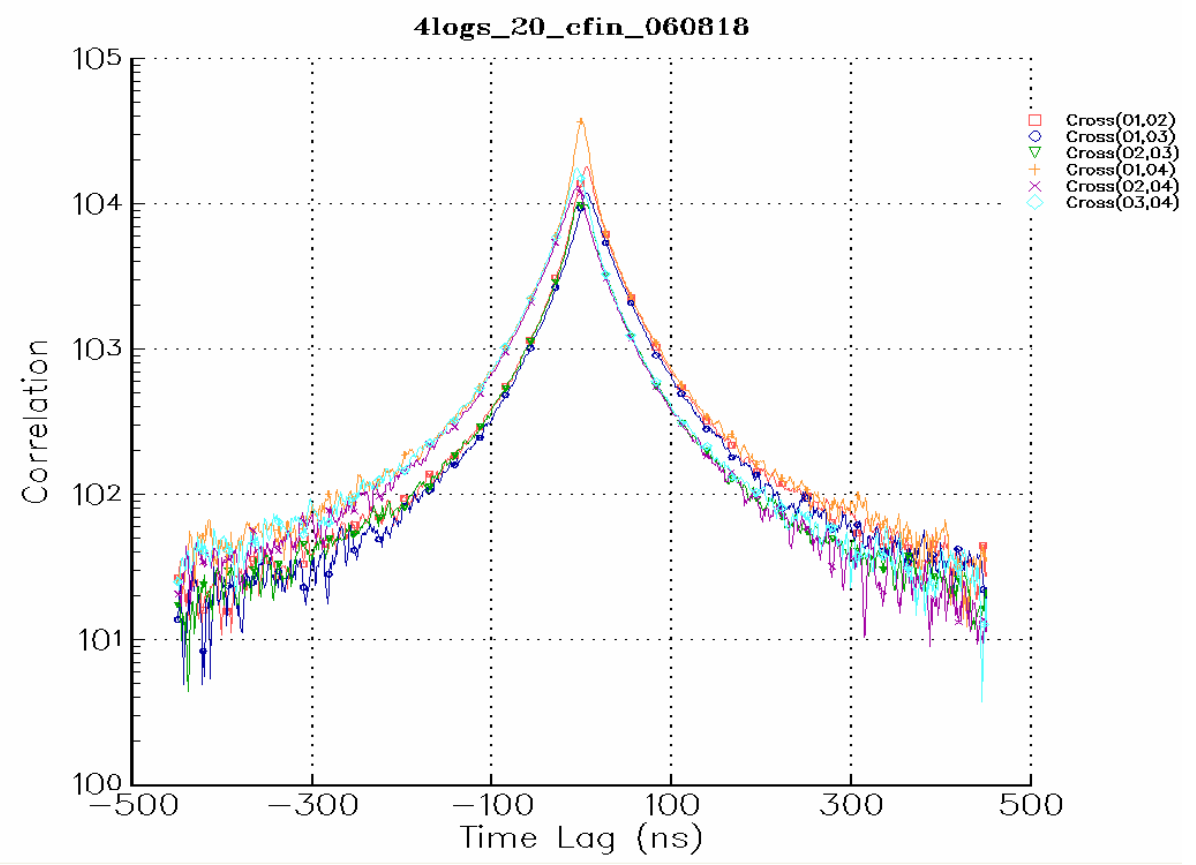

Fig. 6.22. Time distribution of counts in one detector with respect to a previous count in another detector for configuration 20 for all detector combinations.

\subsection{MEASUREMENTS WITH FIVE CASTINGS}

Measurements for five castings with a wide variety of source-detector-casting configurations are listed in detail in Appendix C. A photograph of one of the DT generator-detector-casting measurement configurations (number 34 of table of Appendix C) is given in Fig. 6.23. In this configuration, the DT generator is on the left and the four detectors are adjacent to where two castings are adjacent. The DT generator is located such that the target spot is located on a line between it and the center of the casting in the annular can. The line passes through the point where the two castings near the generator touch. This line is rotated $\sim 4.3^{\circ}$ in the direction of acceleration away from perpendicular to the direction of acceleration. The target spot is $1.5 \mathrm{in}$. from a plane tangent to the two castings near the generator. The casting in the annular can is located in the center of the row of three castings. For measurements with the source internal, the Cf source was located on the axis of the annular can. 


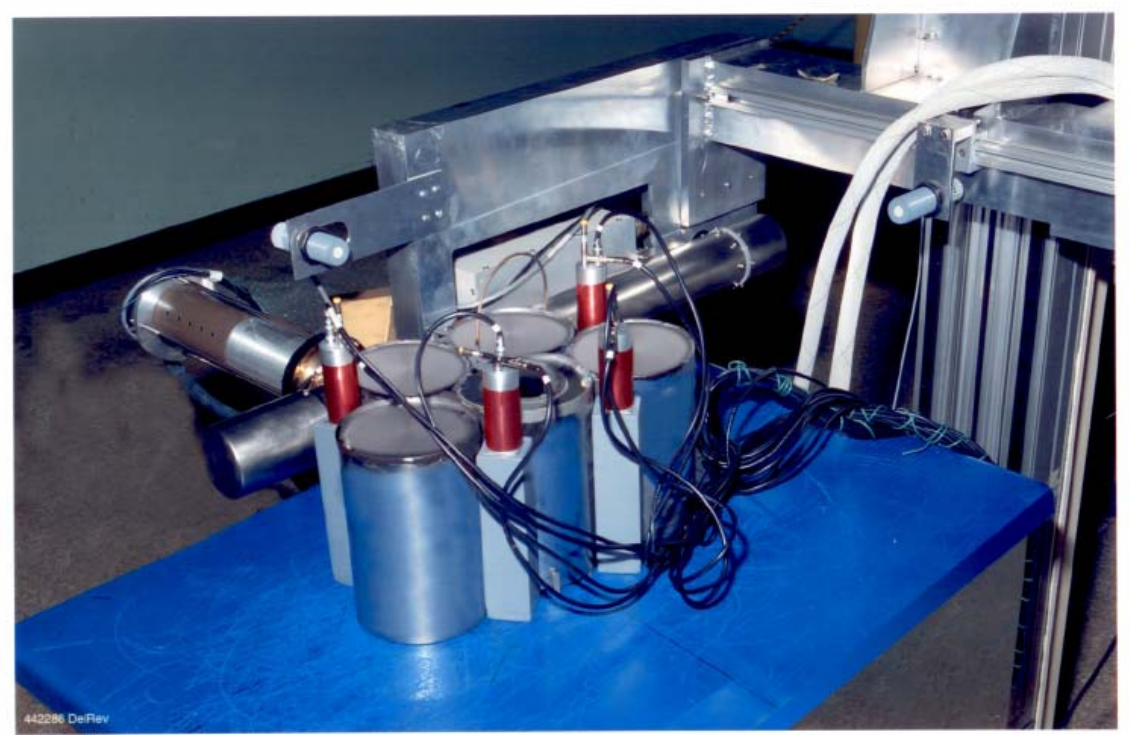

Fig. 6.23. Photograph of one of the DT source-detector-configurations for a measurement with five castings.

The time distribution of counts in each of the detectors after Cf fission for configuration 26 of Appendix $\mathrm{C}$ is shown in Fig. 6.24. The time distribution of detector counts after a count in a previous detector is given in Fig. 6.25 for configuration 26.

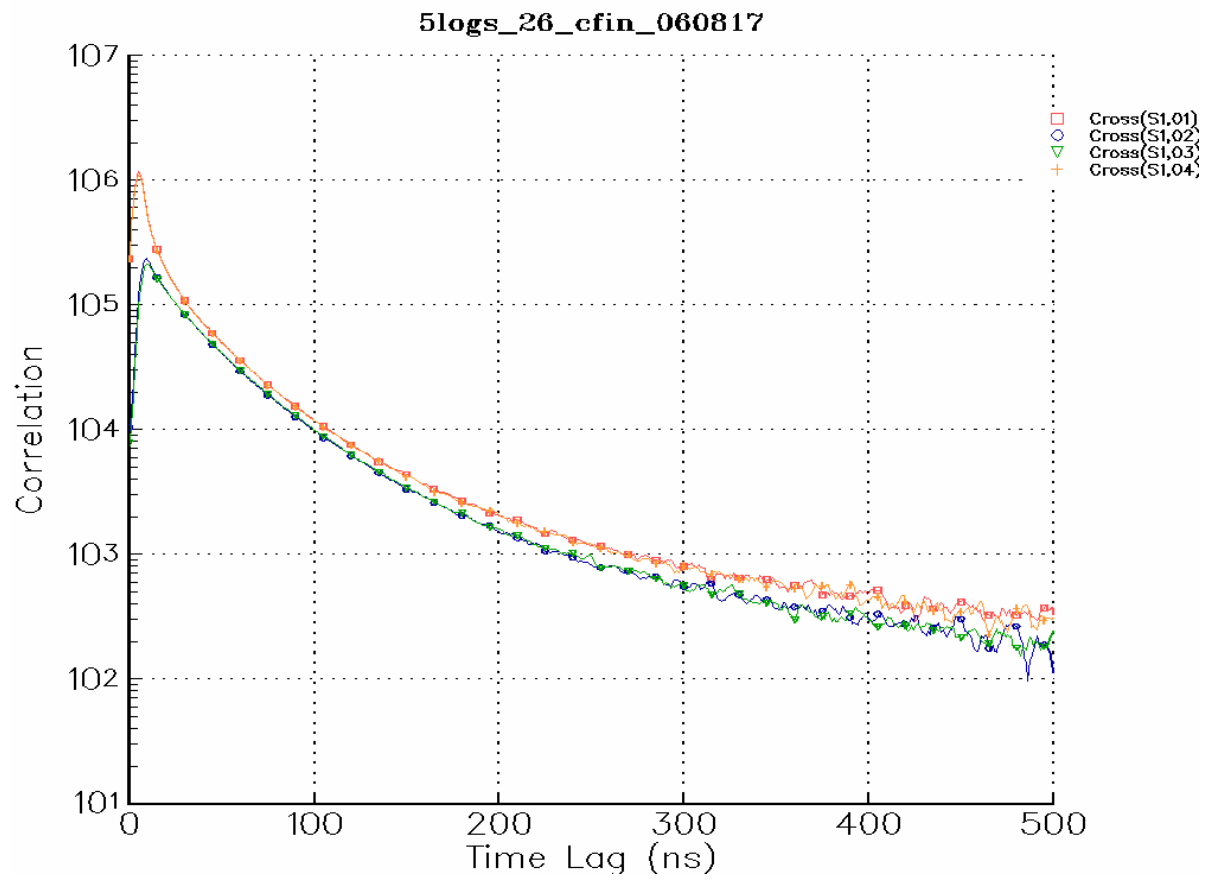

Fig. 6.24. Time distribution of counts in the detectors after $\mathrm{Cf}$ fission for configuration 20 (four castings with Cf internal). 


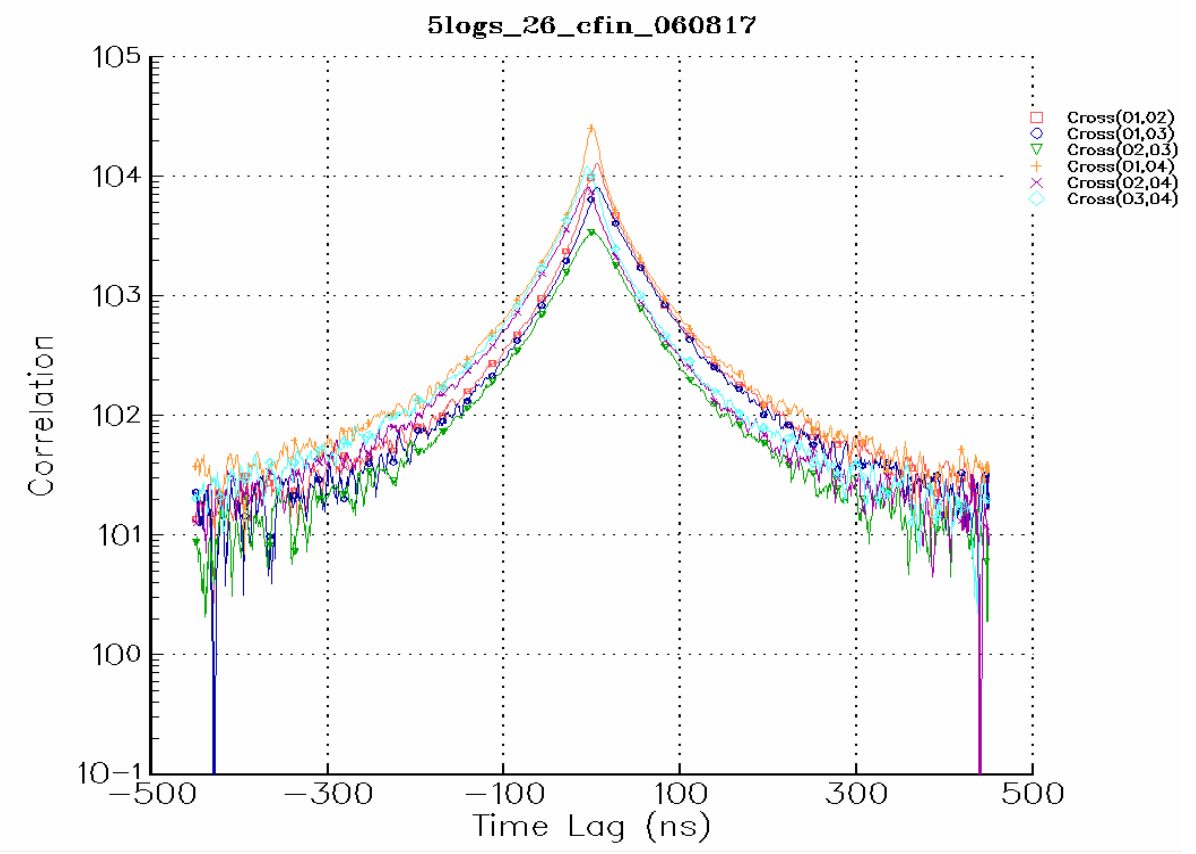

Fig. 6.25. Time distribution of counts in one detector with respect to a previous count in another detector for configuration 26.

\subsection{COMPARISON OF MEASUREMENTS FOR DIFFERENT CASTINGS}

While the previous sections of this report presented data that were measured for each casting configuration, this section presents comparisons for different numbers of measurements and some interesting conclusion about interaction with the floor and walls of the room. The time distributions of the sum of all detector counts (M) after Cf fission are given in Fig. 6.26. The amplitudes of these distributions are not strictly related to the number of castings but are related to the source-detector-casting configurations.

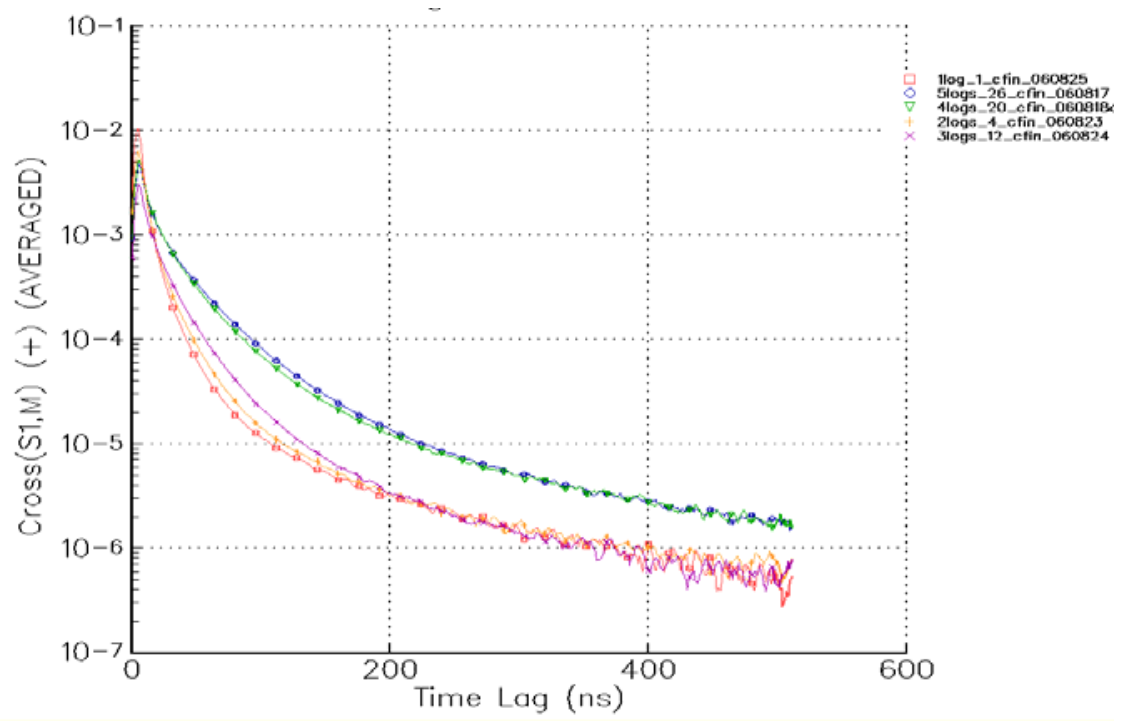

Fig. 6.26. The time distribution of the sum of all detector counts (M) after $\mathrm{Cf}$ fission. 
It is interesting to note that the time response at $\sim 400-500 \mathrm{~ns}$ is the same for different numbers of castings, but the amplitude varies depending on the number of castings, at least between 1, 2, 3 and 4, 5 castings. At these late times, the pulsed fission multiplied casting is acting like a pulsed neutron source for the room. In Fig. 6.27, the time dependence of the response is plotted between 350 and $500 \mathrm{~ns}$.

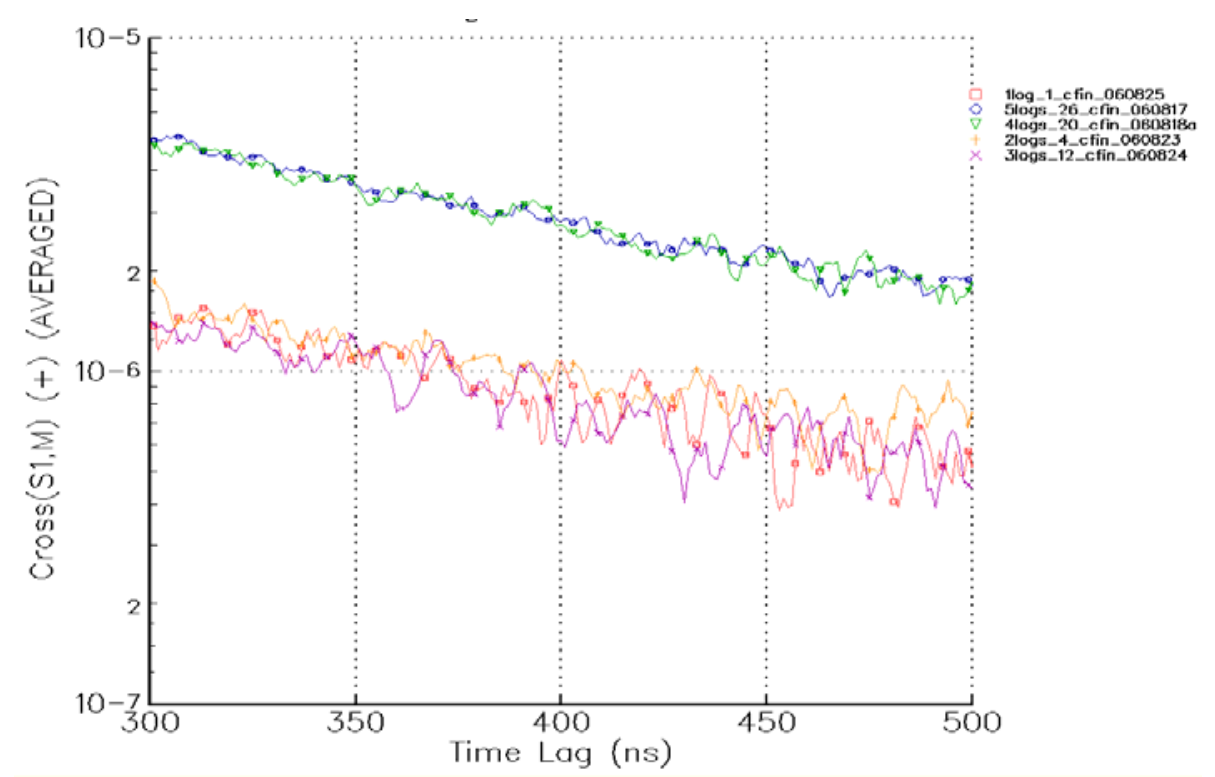

Fig. 6.27. The time distribution of the sum of all detector counts $(M)$ after $\mathbf{C f}$ fission between 350 and $500 \mathrm{~ns}$.

It is clear from this figure that the return from the floor and walls of the room dominate the later time response. This was not true for the premeasurement calculations (Fig. 5.2), which calculated the time distribution of the fission chains internally as a function of casting number but only for times up to $300 \mathrm{~ns}$. This effect is similar to measurements of the correlation between moderated ${ }^{3} \mathrm{He}$ proportional counters and a fast time response source for a HEU metal assembly where the time response is determined by the slowing-down time in the moderator, but the amplitude depends on the fissile mass. The amplitudes measured here do not vary as much with fissile mass because the detector efficiencies for detection of fission in the assemblies vary depending on the number of castings and the configuration of the detectors and source. Where the time response differs substantially for the different number of castings is at the earlier times. These differences are shown in Fig. 6.28. The slopes of the time distributions decrease as the number of castings increases, which is what is expected. The difference in slope between four and five castings is small because the neutron multiplication factor does not change much in going from four to five castings. 


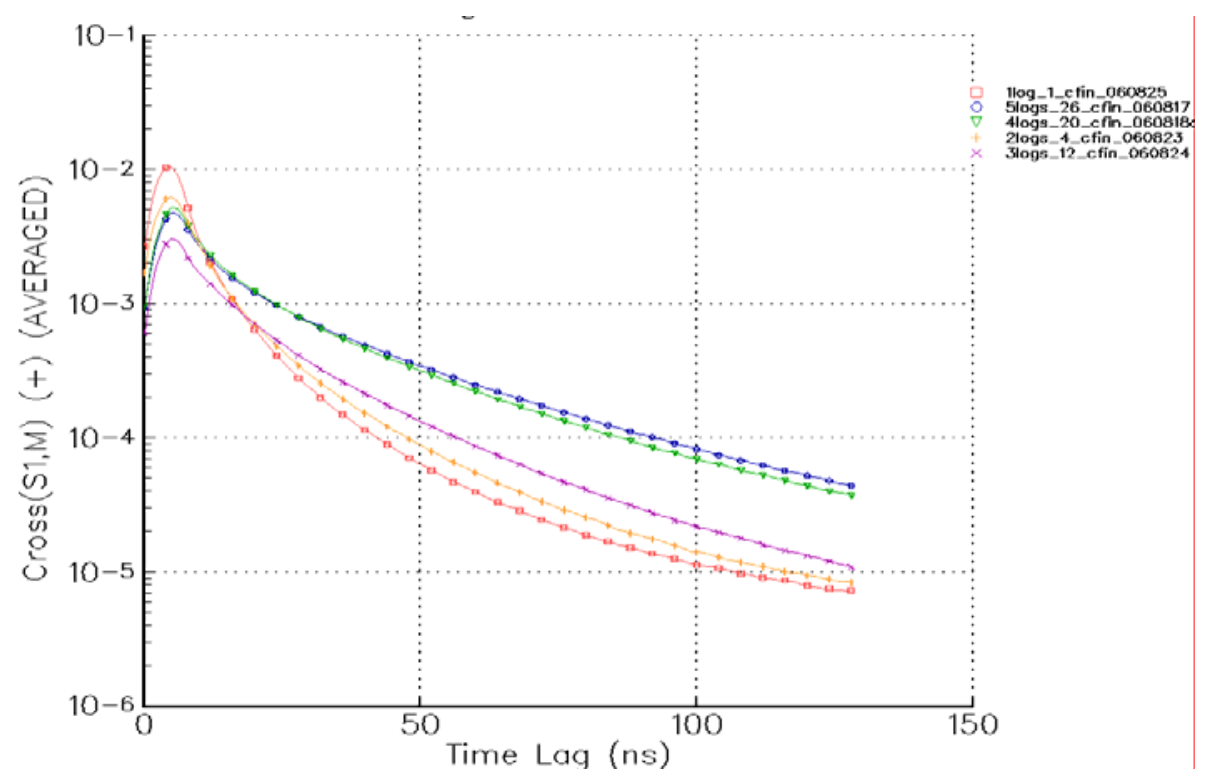

Fig. 6.28. The time distribution of the sum of all detector counts (M) after $\mathrm{Cf}$ fission for the first $125 \mathrm{~ns}$.

Similar results are shown for the DT generator measurements in Fig. 6.29. In this figure, the measurement with no castings was added for comparison. Again, the later times are dominated by the time constants of the casting interaction with the floor and walls of the room. The early time response is given in Fig. 6.30.

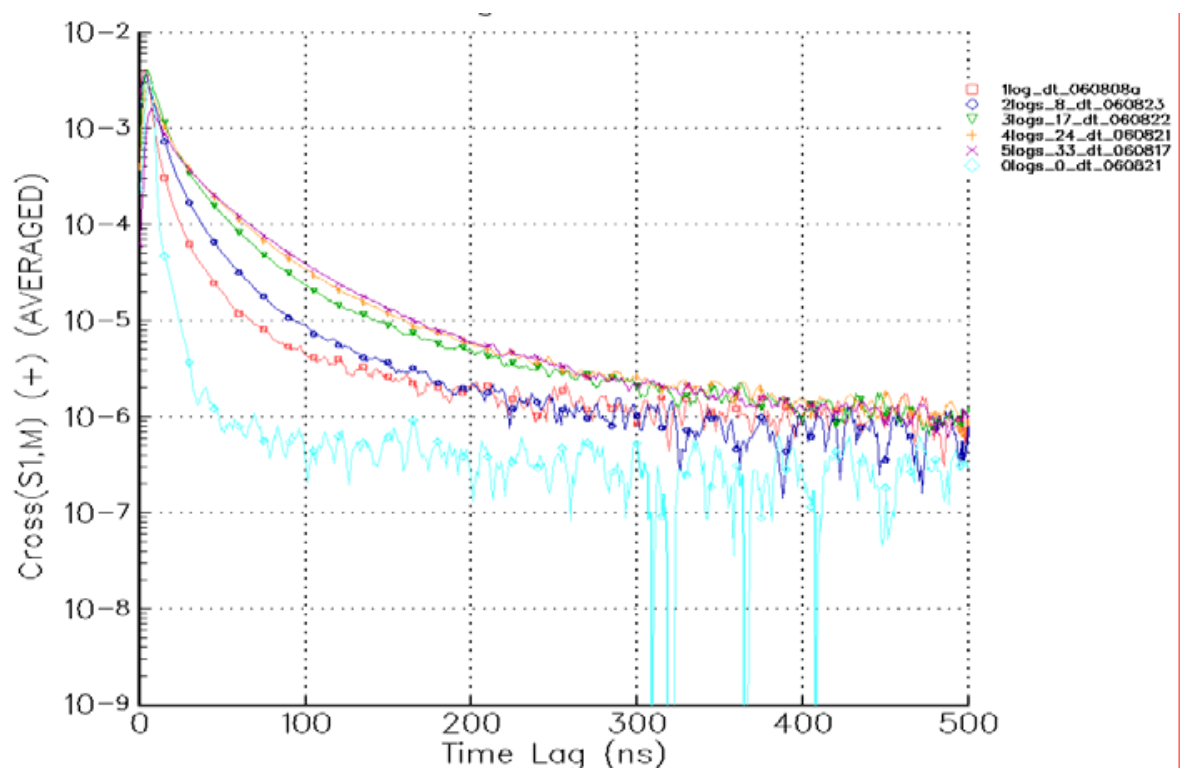

Fig. 6.29. Time distribution of counts after DT reaction summed over all detectors after $\mathrm{Cf}$ fission for none and 1 to 5 casting.

These results indicate that neutron room return effects are significant for these bare configurations. The only way to avoid them is to locate the configurations a considerable distance from the floor and walls of the room. A distance of $3 \mathrm{~m}$ would delay the onset of room return effects with a Cf course to at least 300 ns. Appendix D gives additional information on the gaps between castings cans for various sourcedetector-casting configurations. 


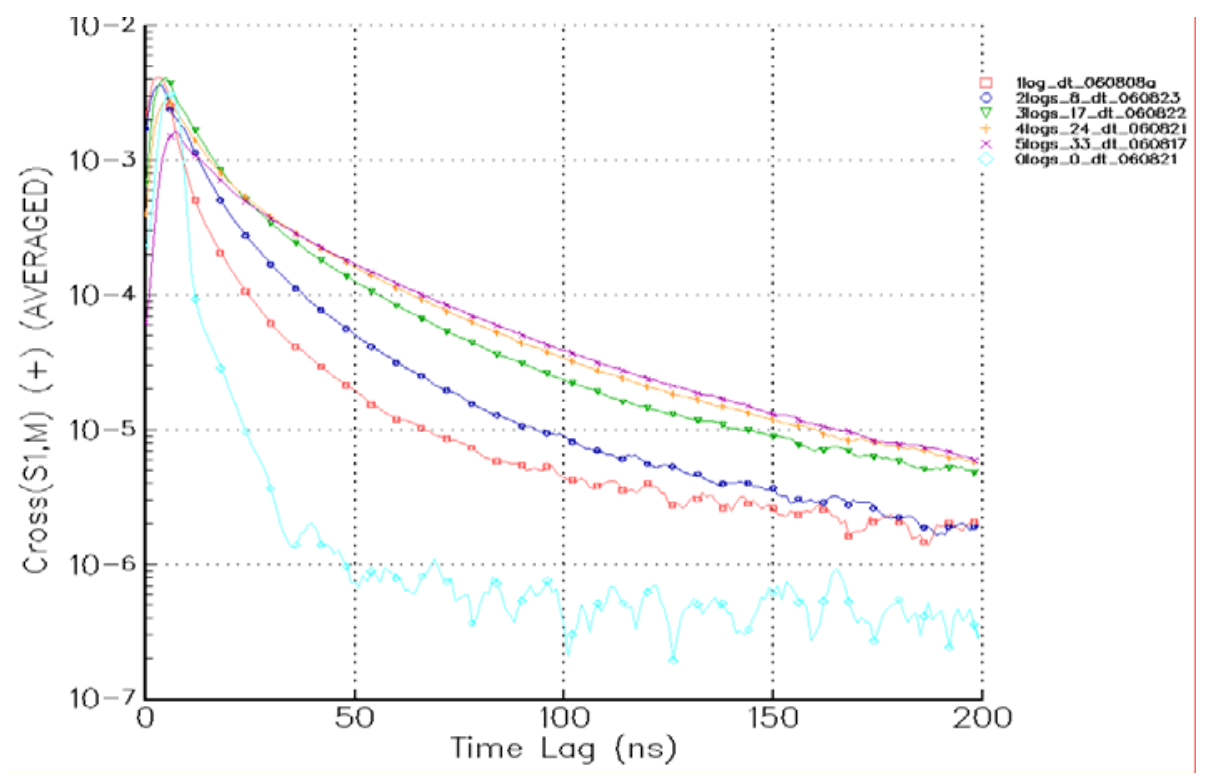

Fig. 6.30. Time distribution of counts after DT reaction summed over all detectors after $\mathrm{Cf}$ fission for none and 1 to 5 casting for times up to $200 \mathrm{~ns}$.

\section{MONTE CARLO SIMULATIONS}

Monte Carlo simulations were performed for some of the measurements and compared to the measured results. In these calculations the detectors were included explicitly in the models. The MCNP-PoliMi requires a detection threshold to simulate the detectors. This threshold was obtained as follows. The timeof-flight measurement in air described in Sect. 4.3 of this report was simulated and the time distribution of counts after $\mathrm{Cf}$ fission shown in Fig. 4.5 was calculated with the threshold varied until the calculations agreed with the measurements. These threshold values were then used for the calculations of the measured time correlation functions. Additional comparisons are given in Appendix E and the Monte Carlo input models for these calculations are given in Appendix F. In these calculations, the walls and floor of the room and the steel table on which the castings were located were included in the calculations. The first five sections (Sects. 7.1-7.4) present the comparisons of the calculated time response with the measured over the full time response (500 ns). These comparisons are followed by a discussion of the differences (Sect. 7.6).

\subsection{ONE CASTING WITH CF SOURCE INTERNAL}

The Cf source-detector-casting configuration for a measurement with the $\mathrm{Cf}$ internally located on the axis of the casting is given in Fig. 7.1. The time distribution of coincidences between detector 1 and the $\mathrm{Cf}$ source is compared to that measured in Fig. 7. 2. The agreement between calculated and measured time distribution of coincidences is good except for the early times up to $100 \mathrm{~ns}$. Similar plots of the results for detector 4 are given in Fig. 7.3, where again the agreement between calculations is similar. There was also similar agreement for detectors 2 and 3 . 


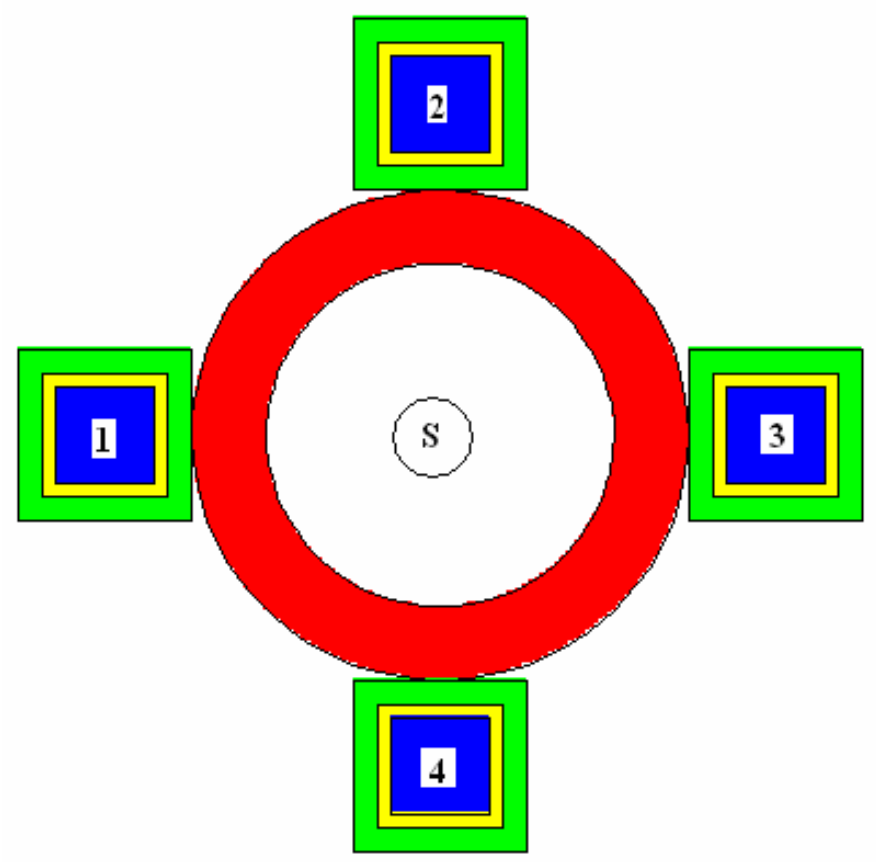

CONFIGURATION 1

Fig. 7.1. Top view Monte Carlo geometry for calculations of a single casting with the $\mathrm{Cf}$ source internal.

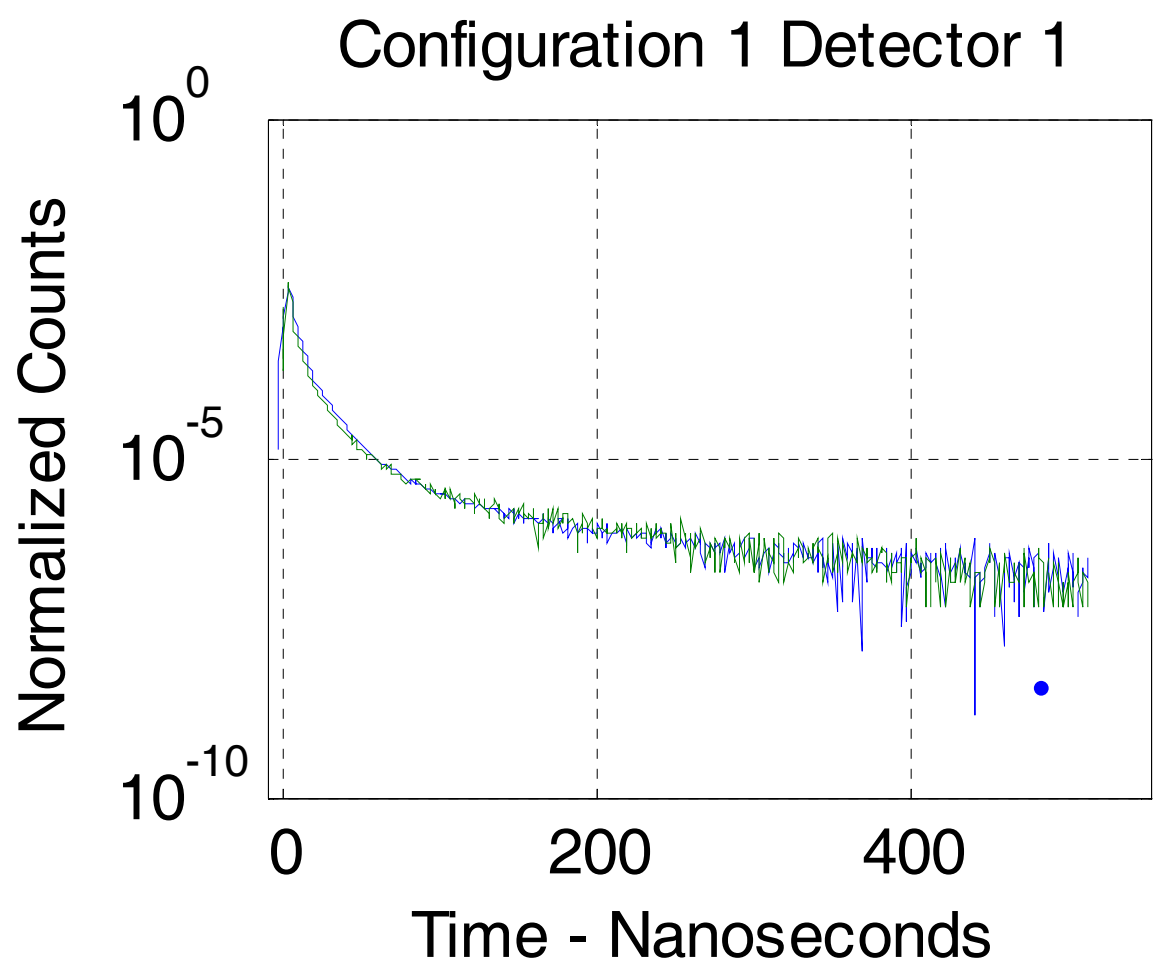

Fig. 7.2. Time distribution of counts in detector 1 after $\mathbf{C f}$ fission in counts per $\mathbf{C f}$ fission. The blue curve is measured and the other is calculated. 


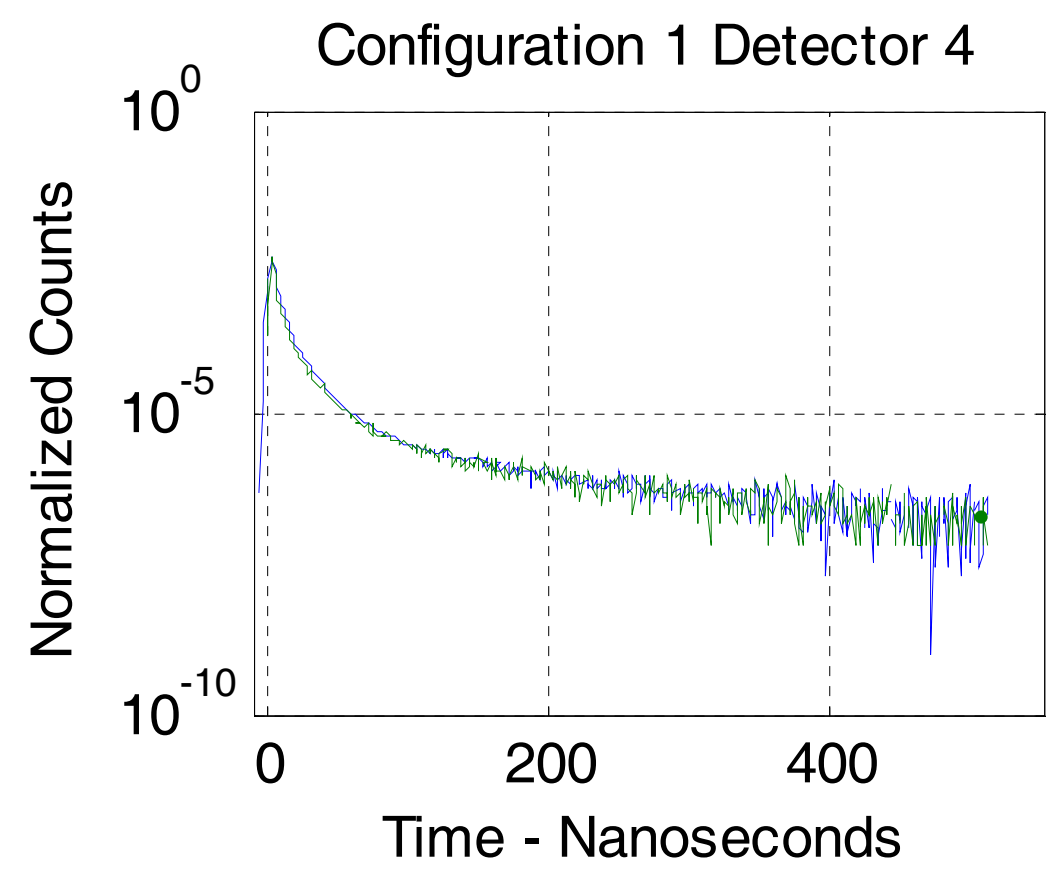

Fig. 7.3. Time distribution of counts in detector 4 after $\mathbf{C f}$ fission in counts per $\mathbf{C f}$ fission. The blue curve is measured and the other is calculated.

The time distribution of counts in the detector after $\mathrm{Cf}$ fission for the first $200 \mathrm{~ns}$ is given in Fig. 7.4 and that for the first $30 \mathrm{~ns}$ is given in Fig. 7.5. The calculation considerably underpredicts the measurements for the first $60 \mathrm{~ns}$ and correctly predicts the latter part of the time decay. This can be seen clearly in the data of Fig. 7.5 for the first $30 \mathrm{~ns}$. The disagreement of the first $30 \mathrm{~ns}$ varies for the four detectors because the detection efficiencies are slightly different from measurements, or the $\mathrm{Cf}$ source is not exactly on the axis of the casting. The underprediction of the calculations for the early measurement times increases with the number of castings. 


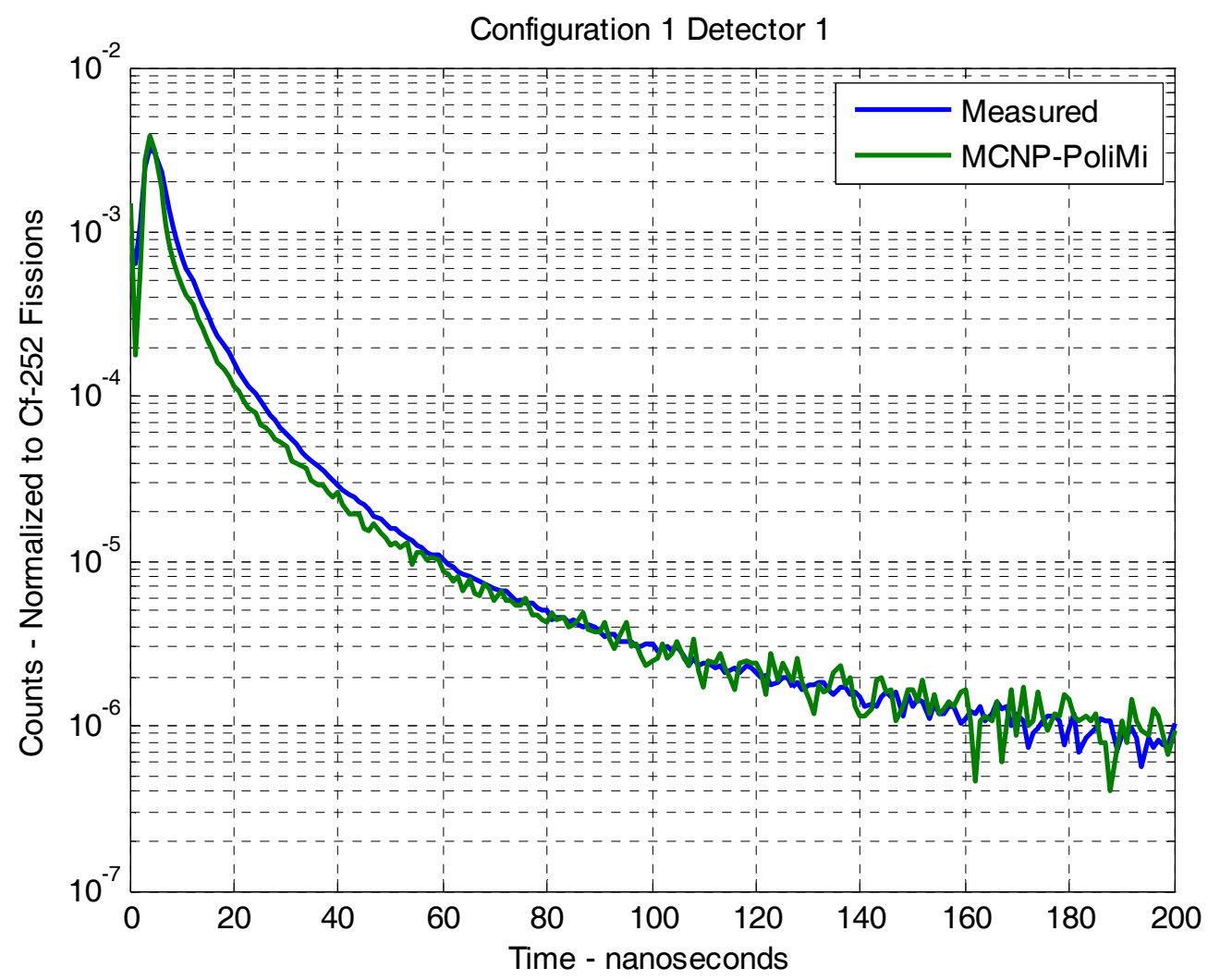

Fig. 7.4. Time distribution of counts in detector 1 after $\mathrm{Cf}$ fission in counts per $\mathrm{Cf}$ fission for the first 200 ns. Blue curve is measured and the other is calculated.

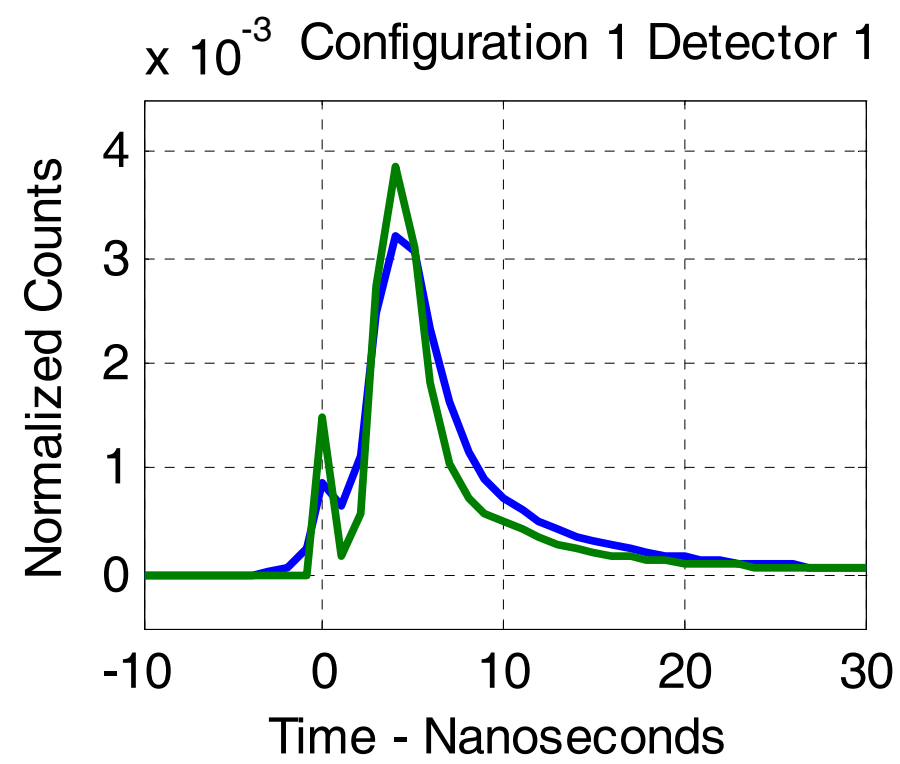

Fig. 7.5. Time distribution of counts in detector 1 after $\mathrm{Cf}$ fission in counts per Cf fission. For the first 30 ns. Blue curve is measured and the other is calculated. 


\subsection{TWO CASTINGS}

A sketch of the calculated configuration for two castings is shown in Fig. 7.6. The time distribution of coincidences between detector 1 and the Cf source is compared to that measured in Fig. 7.7. The agreement between calculated and measured time distribution of coincidences is very good except for the early times up to $100 \mathrm{~ns}$.

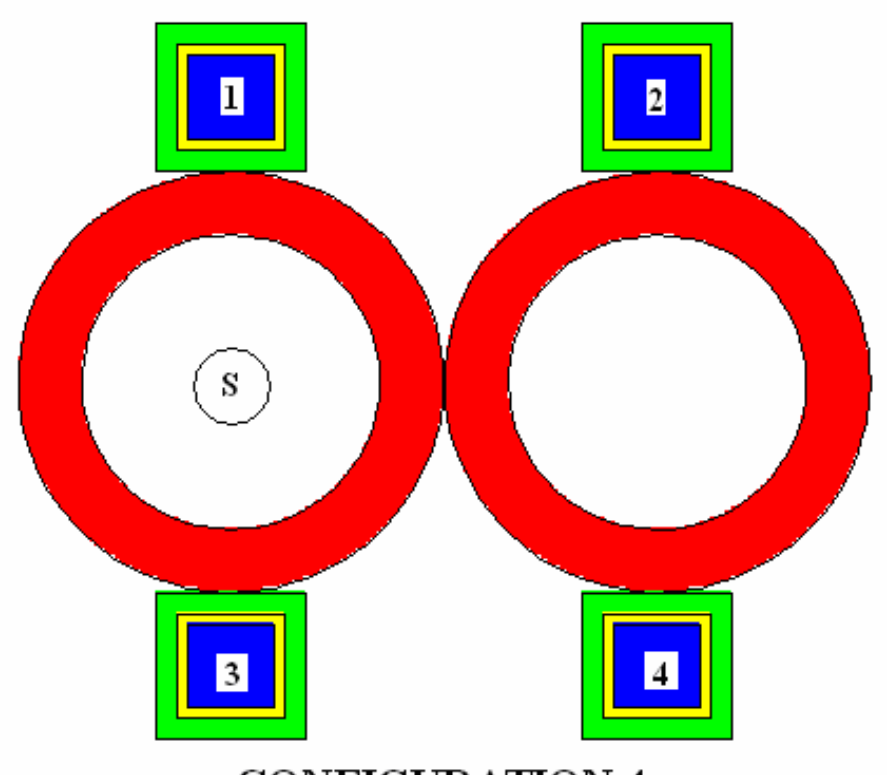

CONFIGURATION 4

Fig. 7.6. Top view Monte Carlo geometry for calculations of a two casting with the Cf source internal. 


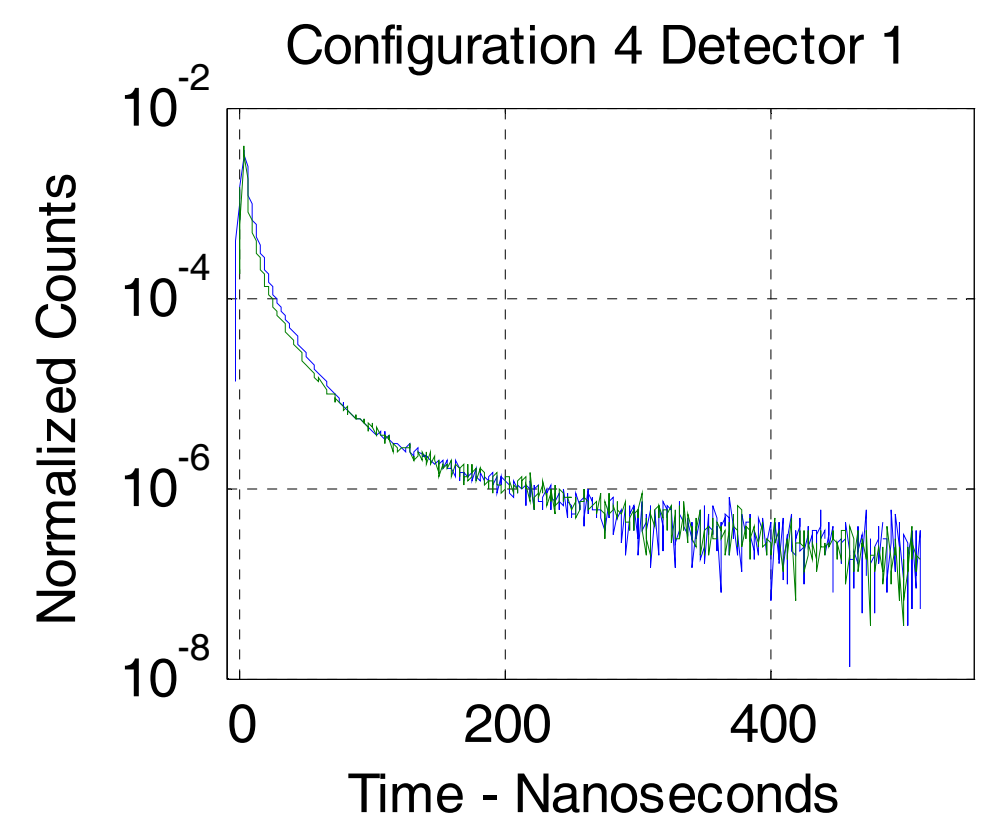

Fig. 7.7. Time distribution of counts in detector 1 after $\mathbf{C f}$ fission in counts per $\mathbf{C f}$ fission. The blue curve is measured and the other is calculated.

\subsection{THREE CASTINGS}

A sketch of the calculated configuration for three castings is shown in Fig. 7.8. The time distributions of coincidences between detectors 3 and 4 and the Cf source are compared to those measured in Figs. 7.9 and 7.10. The agreement between calculated and measured time distribution of coincidences is very good except for the early times up to $100 \mathrm{~ns}$. 


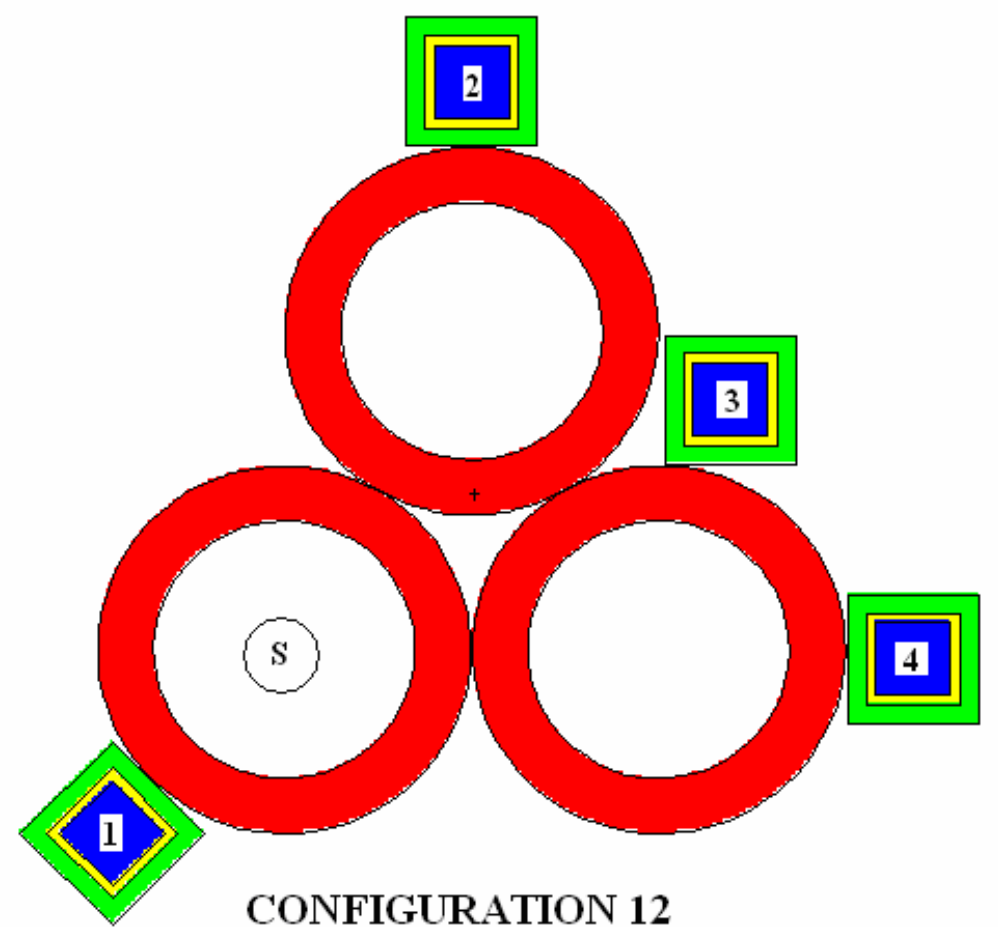

Fig. 7.8. Top view Monte Carlo geometry for calculations of a three casting with the $\mathrm{Cf}$ source internal.

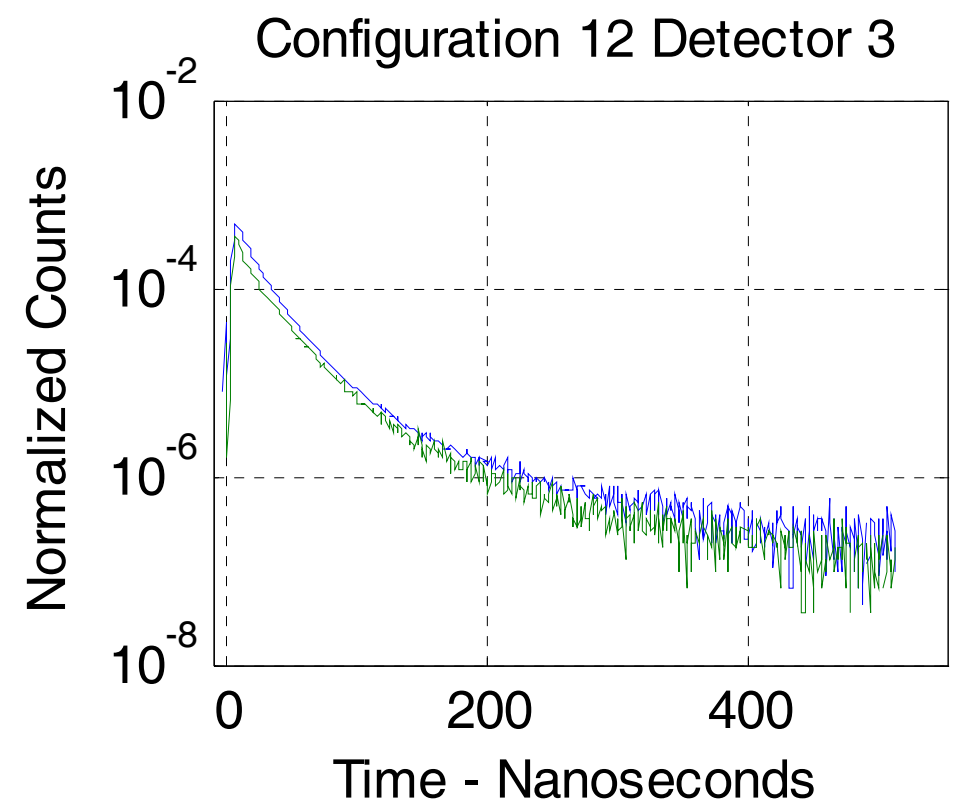

Fig. 7.9. Time distribution of counts in detector 3 after $\mathbf{C f}$ fission in counts per $\mathbf{C f}$ fission. The blue curve is measured and the other is calculated. 


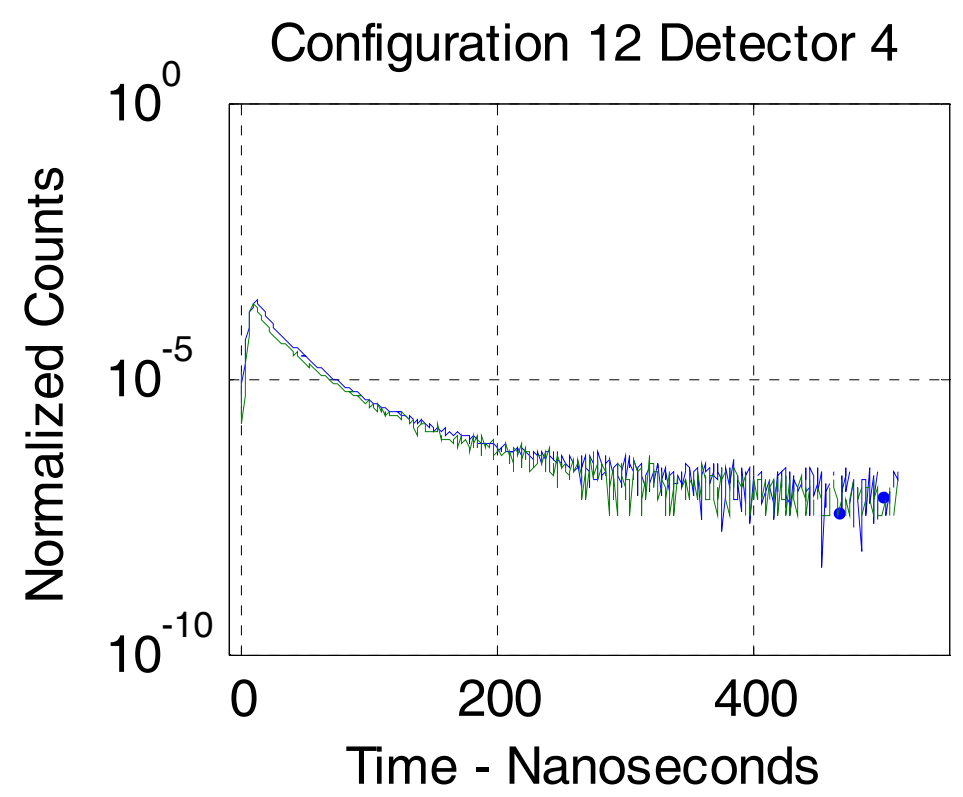

Fig. 7.10. Time distribution of counts in detector 4 after $\mathbf{C f}$ fission in counts per $\mathbf{C f}$ fission. The blue curve is measured and the other is calculated.

\subsection{FOUR CASTINGS}

A sketch of the calculated configuration for four castings is shown in Fig. 7.11. The time distributions of coincidences between detectors 1 and 2 and the $\mathrm{Cf}$ source are compared to those measured in Figs. 7.12 and 7.13. The agreement between calculated and measured time distribution of coincidences is very good except for the early times up to $100 \mathrm{~ns}$. 


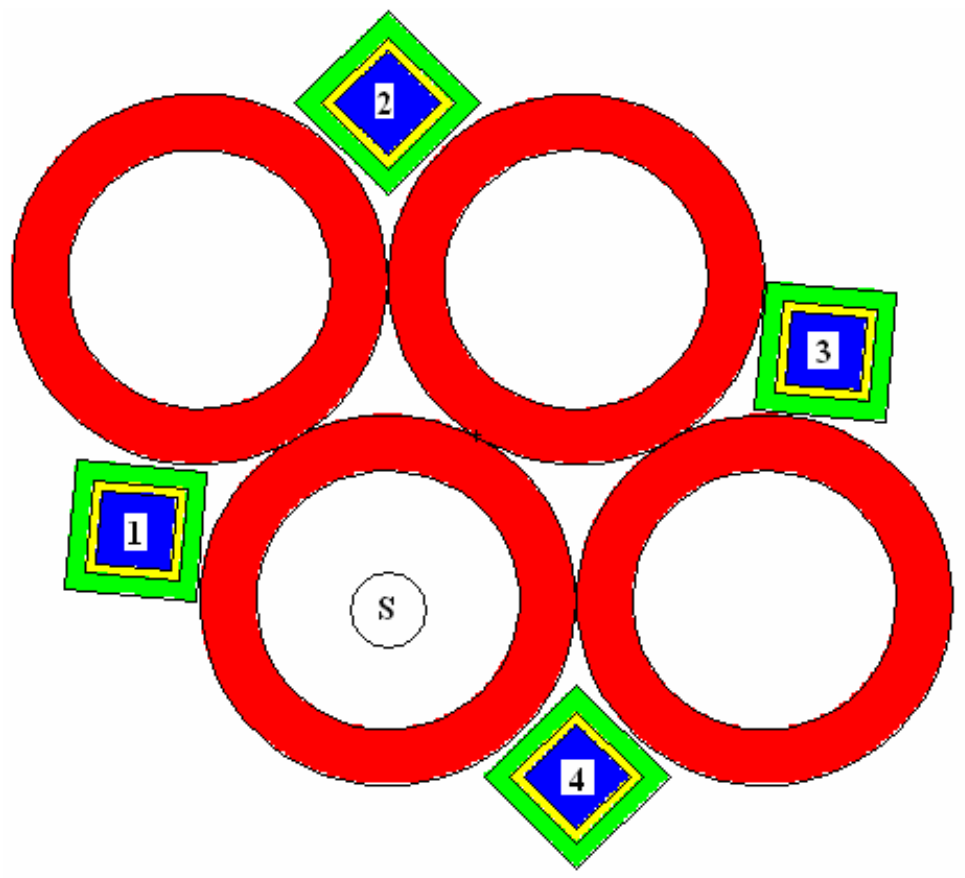

CONFIGURATION 20

Fig. 7.11. Top view Monte Carlo geometry for calculations of four casting with the Cf source internal.

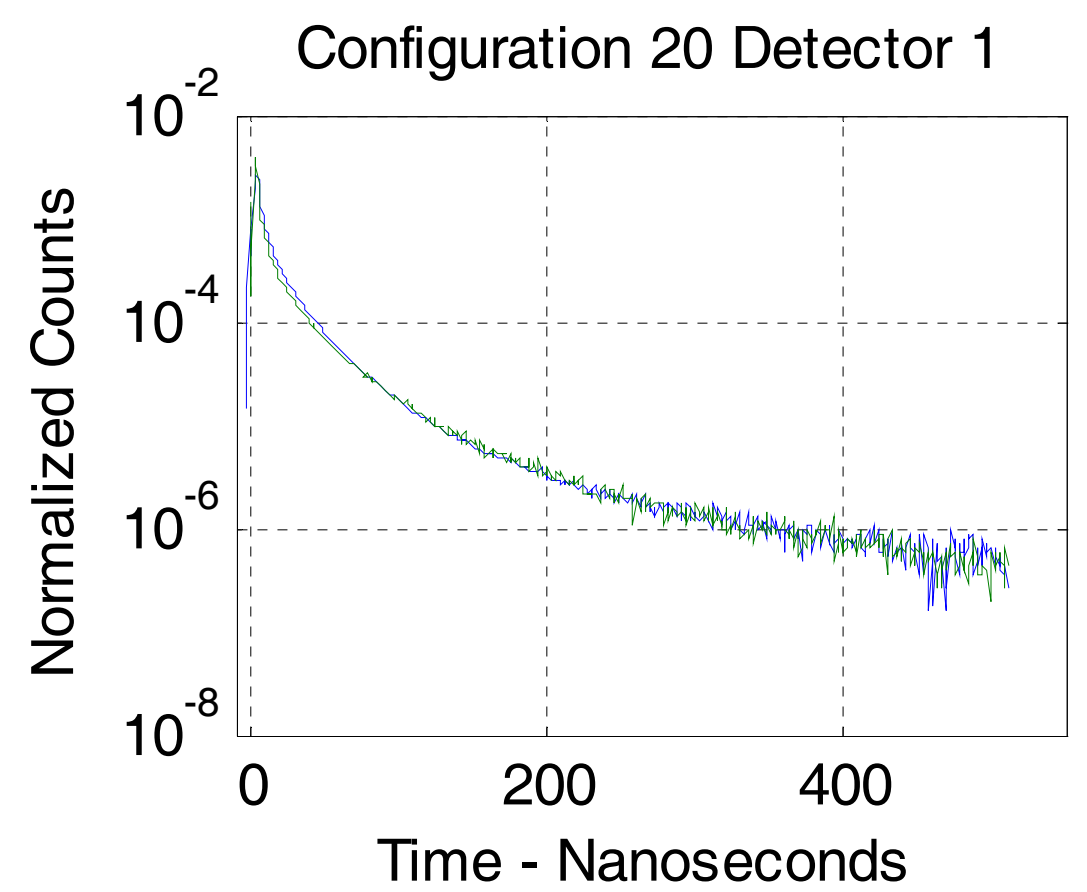

Fig. 7.12. Time distribution of counts in detector 1 after $\mathrm{Cf}$ fission in counts per Cf fission. The blue curve is measured and the other is calculated. 


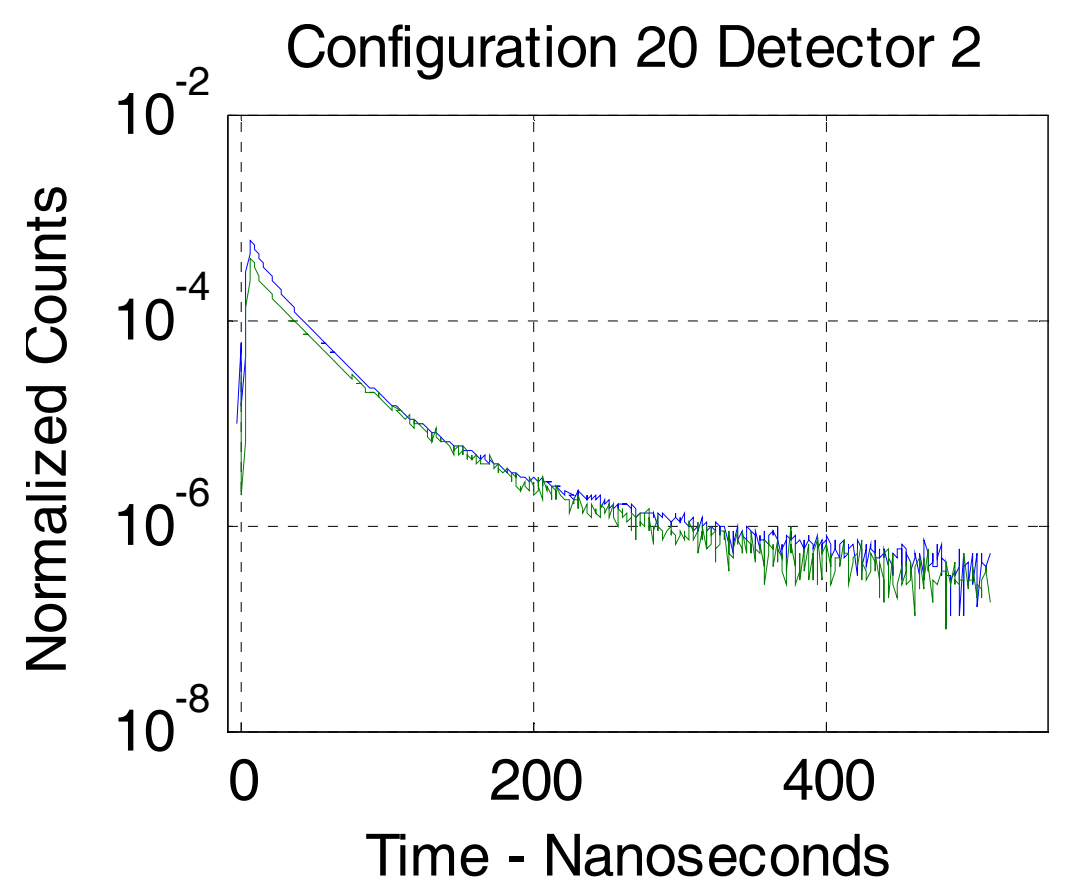

Fig. 7.13. Time distribution of counts in detector 2 after $\mathrm{Cf}$ fission in counts per $\mathbf{C f}$ fission. The blue curve is measured and the other is calculated.

\subsection{FIVE CASTINGS}

A sketch of the calculated configuration for five castings is shown in Fig. 7.14. The time distributions of coincidences between detectors 1 and 3 and the Cf source are compared to those measured in Figs. 7.15 and 7.16. The agreement between calculated and measured time distribution of coincidences is very good except for the early times up to $100 \mathrm{~ns}$. Similar data for detector 2 for the first $200 \mathrm{~ns}$ is given in Fig. 7.17, where the underestimation of the calculated result is the largest. 


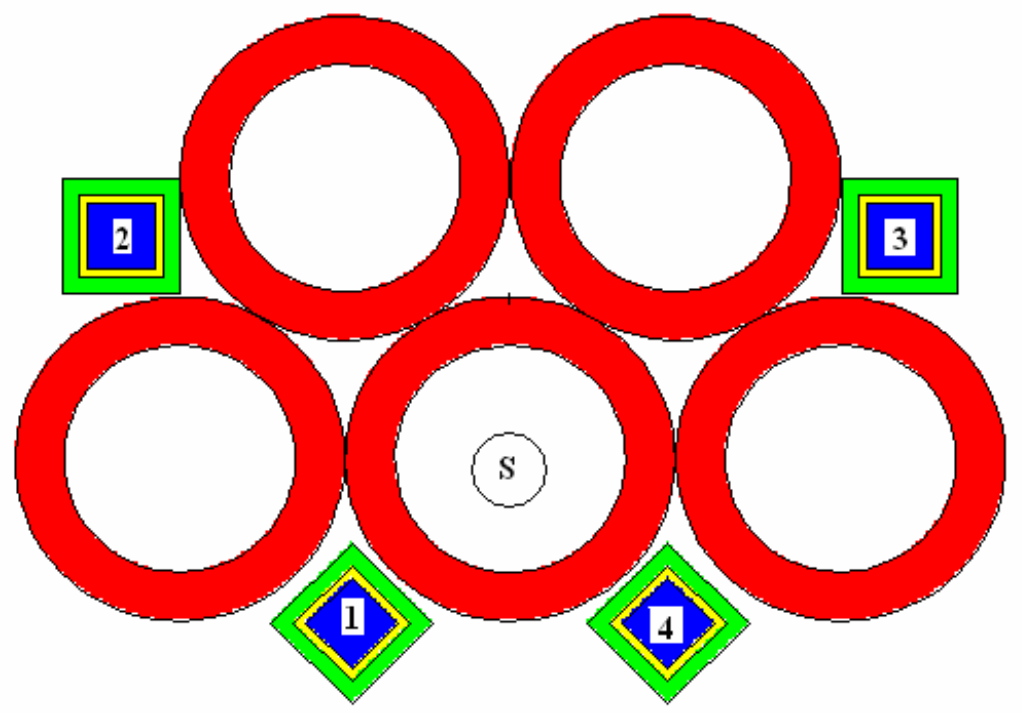

CONFIGURATION 26

Fig. 7.14. Top view Monte Carlo geometry for calculations of five castings with the $\mathrm{Cf}$ source internal.

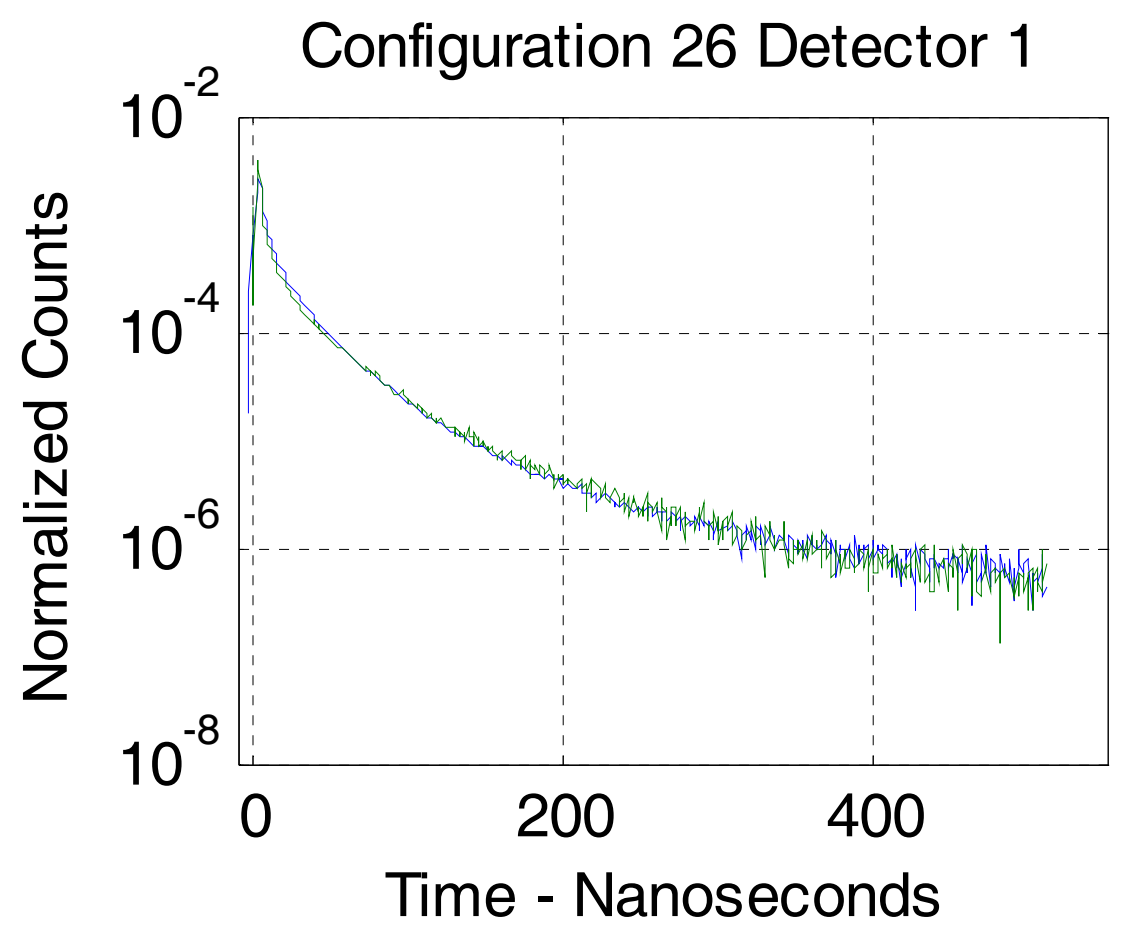

Fig. 7.15. Time distribution of counts in detector 1 after $\mathbf{C f}$ fission in counts per Cf fission. The blue curve is measured and the other is calculated. 


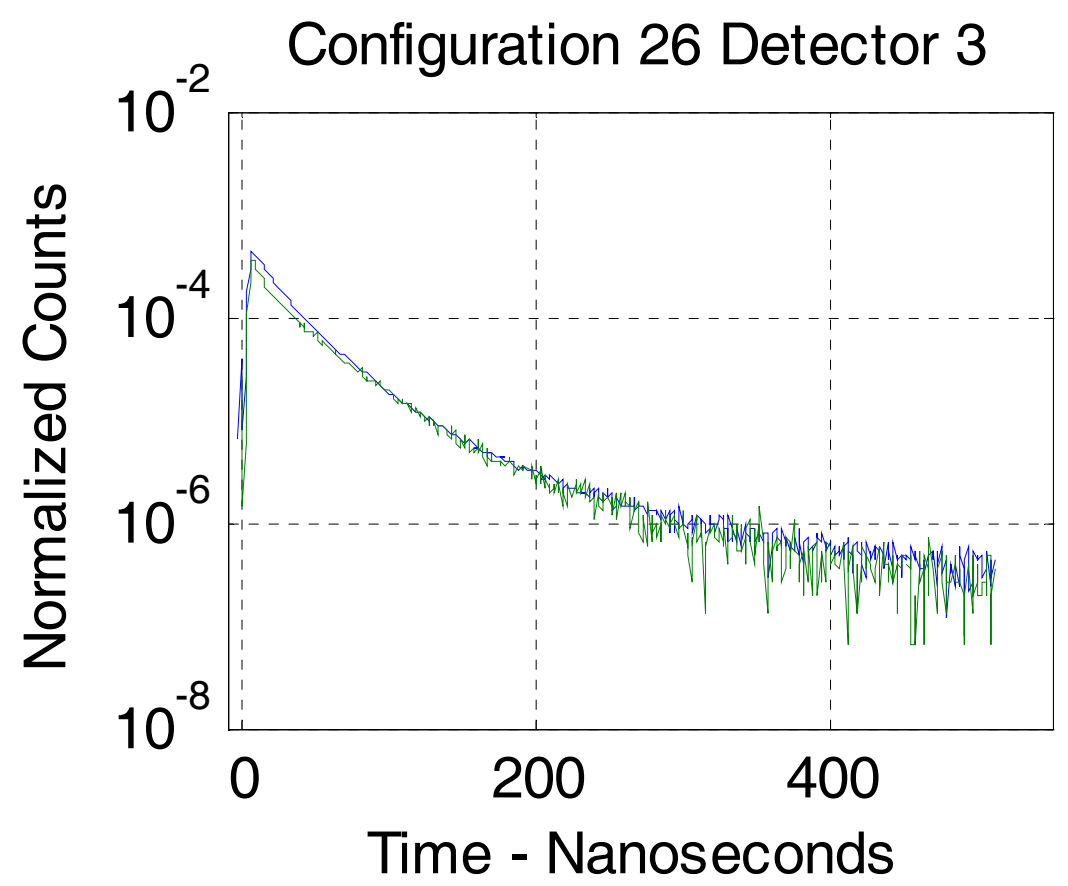

Fig. 7.16. Time distribution of counts in detector 3 after $\mathrm{Cf}$ fission in counts per $\mathrm{Cf}$ fission. The blue curve is measured and the other is calculated.

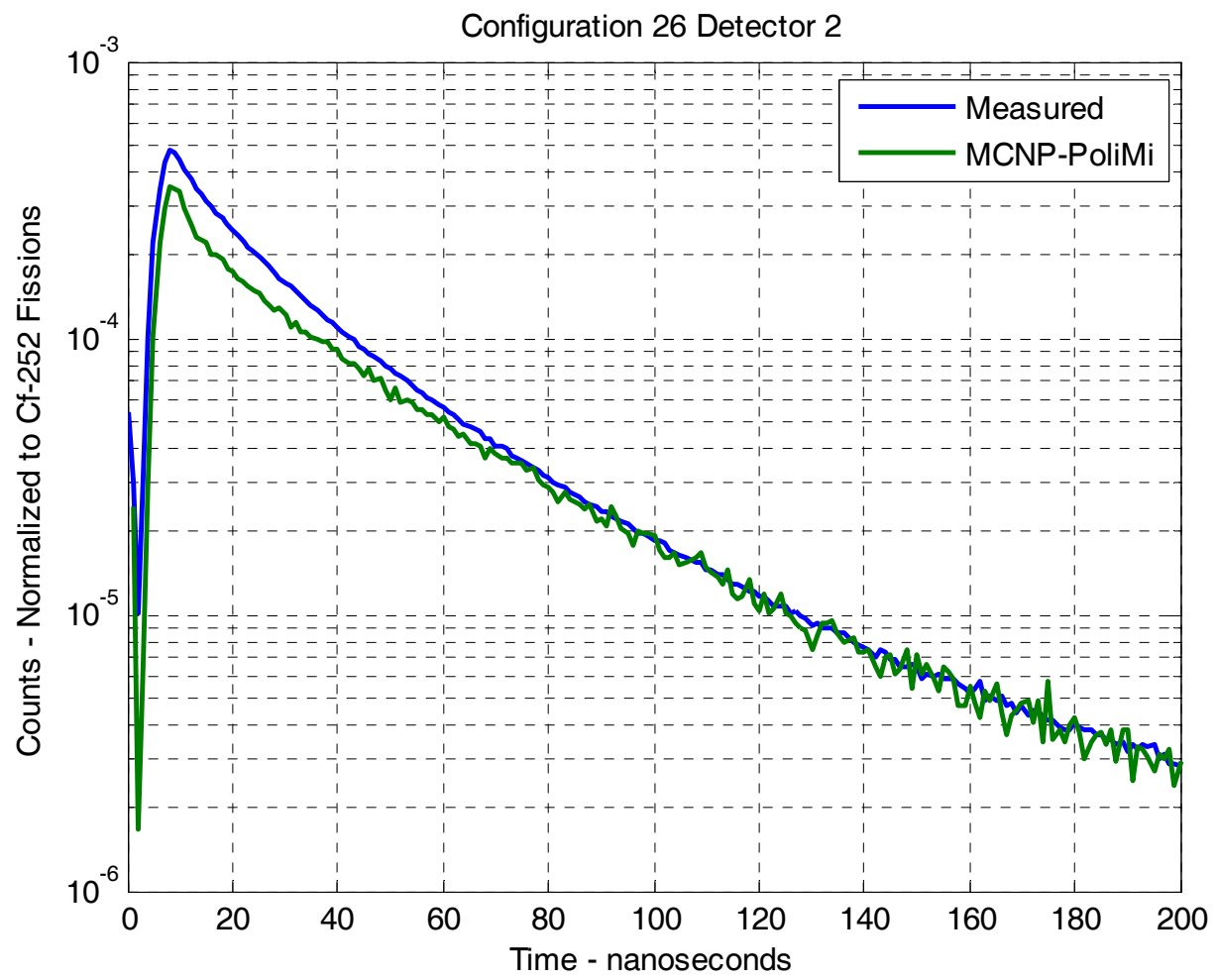

Fig. 7.17. Time distribution of counts in detector $\mathbf{2}$ for 200 ns after $\mathbf{C f}$ fission in counts per Cf fission. The blue curve is measured and the other is calculated. 


\subsection{POST EXPERIMENT ROOM RETURN CALCULATIONS}

The calculations in Fig 5.1 were repeated out to $1000 \mathrm{~ns}$, and the results of the time distributions of internal fission with respect to the time of the $\mathrm{Cf}$ spontaneous fission event are plotted in Fig. 7.18. Again, the effects of neutron room return appear at later times because the late time response is determined predominately by the room. These calculations included the steel support table and the wall, floor, and ceiling of the room. The calculation was repeated without external reflection effects, and the percentage of the signal that is associated with neutron room return effect is plotted as a function of time in Fig 7.19. Calculated room return effects appear as early as $100 \mathrm{~ns}$ but are significant for later times. The percentage of the fissions in the system associated with room return neutrons increases with time and with the number of castings. The increase associated with the number (size) of castings results from the reflected neutrons seeing a larger target to intercept. The solid angle subtended by the assembly for a reflected neutron is larger for the larger assemblies. At later times the percentages of fissions associated with room return neutrons are 20,30, and 50\% for the 1, 4, and 6 casting assemblies, respectively. The room return neutrons initially appear at $\sim 100 \mathrm{~ns}$, and their affect increases with time because the fission chain population is decreasing.

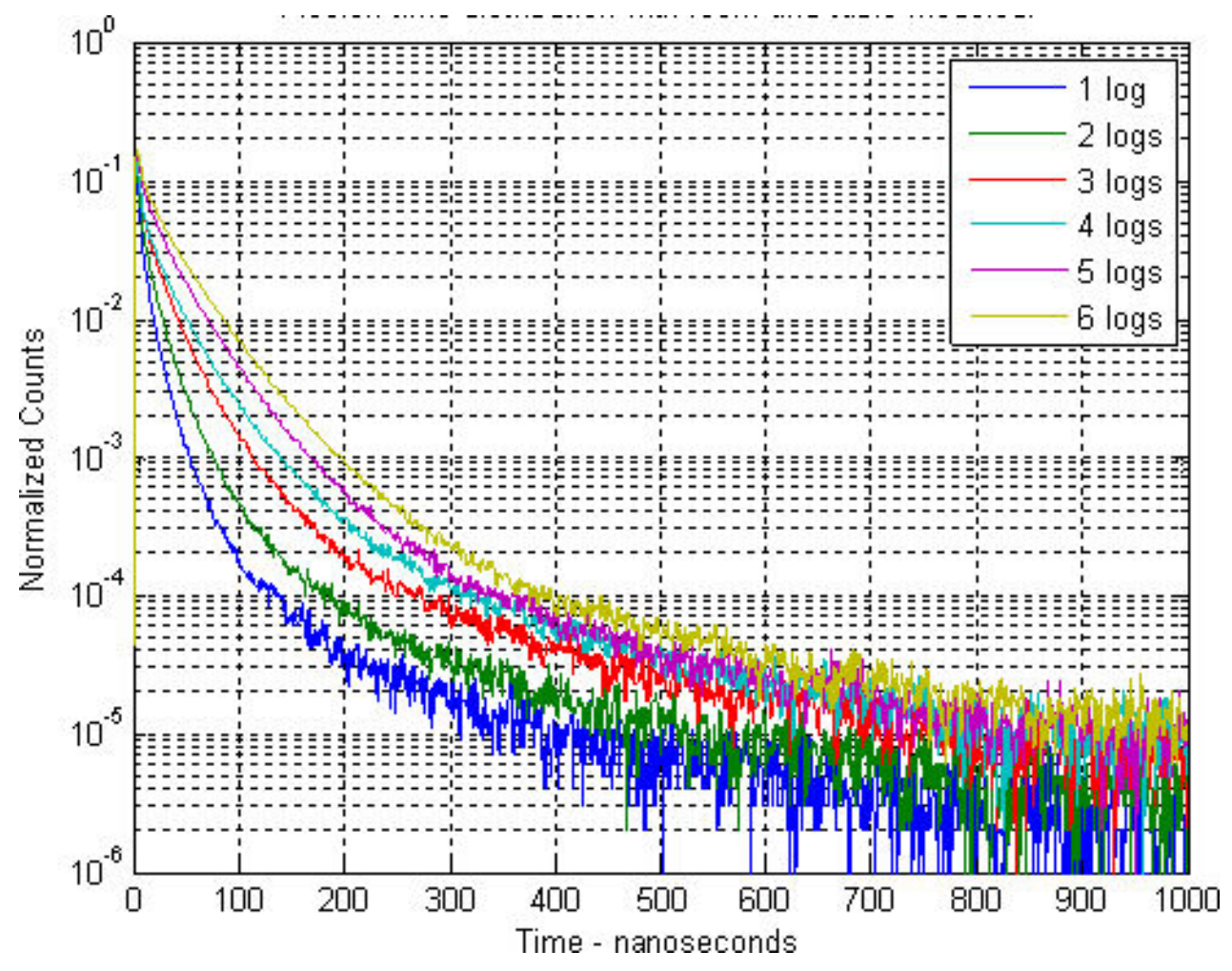

Fig. 7.18. Calculated fission time distribution after $\mathrm{Cf}$ fission with room and table modeled. 


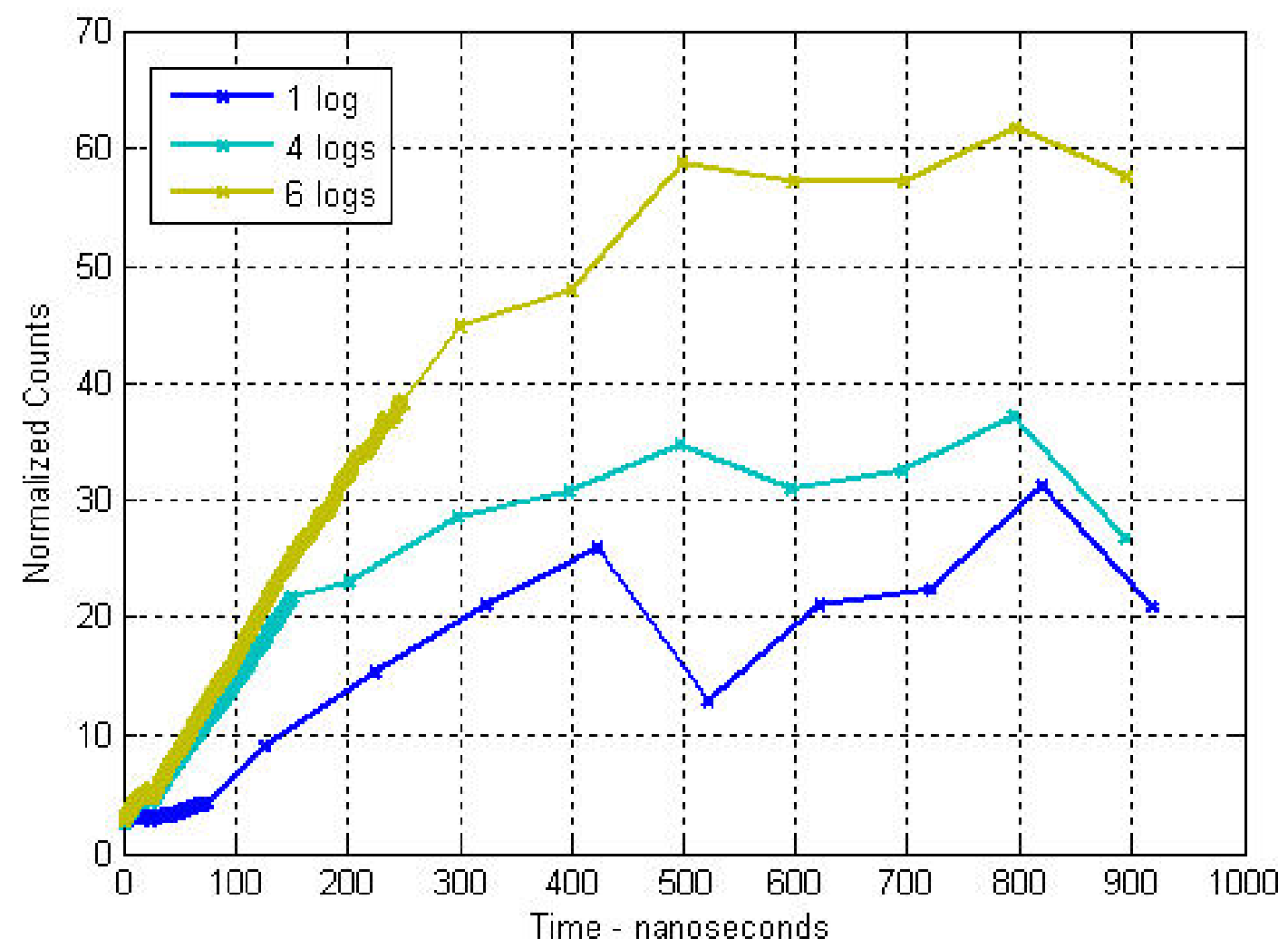

Fig. 7.19. Percent difference between model with room/table modeled and without room/table modeled. Later points averaged over $100 \mathrm{~ns}$.

\section{CONCLUSIONS}

These carefully performed and documented measurements with unreflected and unmoderated HEU castings can be used to benchmark calculational methods for the time decay of the fission chain multiplication process as measured with small (to minimize detector effects) detectors in close proximity to HEU metal castings. The use of a DT generator provided no additional information beyond that provided by a time-tagged $\mathrm{Cf}$ source inside one of the castings in the array of castings up to five. Neutron room return appears as early as $100 \mathrm{~ns}$ and significantly affects the time behavior after $400 \mathrm{~ns}$. The calculations with MCNP-PoliMi the measurements for the early time in the fission chain multiplication process but agreed extremely well for time after $100 \mathrm{~ns}$ after the initiation of the fission chain by a timetagged spontaneous fission in a $\mathrm{Cf}$ ionization chamber.

\section{REFERENCES}

1. J.T. Mihalczo, "The Use of ACalifornium-252 as a Randomly Pulsed Neutron Source for PromptNeutron Decay Measurements," Nucl. Sci. \& Engr. 53, 393-414 (1974).

2. J.T. Mihalczo, J.A. Mullens, J.K. Mattingly, T.E. Valentine, "Physical Description of Nuclear Materials Identification System (NMIS) Signatures," Nucl. Inst. and Meth. A 450, 531 (2000).

3. Thermo Fisher Scientific Electron Corp., "API120 D-T Neutron Generator User Manual,” Colorado Springs, CO 80919, www.thermo.com/neutrongenerators. 
4. S.A. Pozzi, E. Padovani, and M. Marseguerra, "MCNP-PoliMi: A Monte Carlo Code for Correlation Measurements," Nuclear Instruments and Methods in Physics Research Section A, $513 / 3$ pp. 550-558, 2003. 
APPENDIX A: URANIUM ISOTOPICS AND IMPURITY ANALYSES 


\section{APPENDIX A: URANIUM ISOTOPICS AND IMPURITY ANALYSES}

The spectroscopic impurity analyses and isotopic analyses for the five casting are presented in Table A.1. 
Table A.1. Summary of Isotopic and Chemical Data 5/31/2006

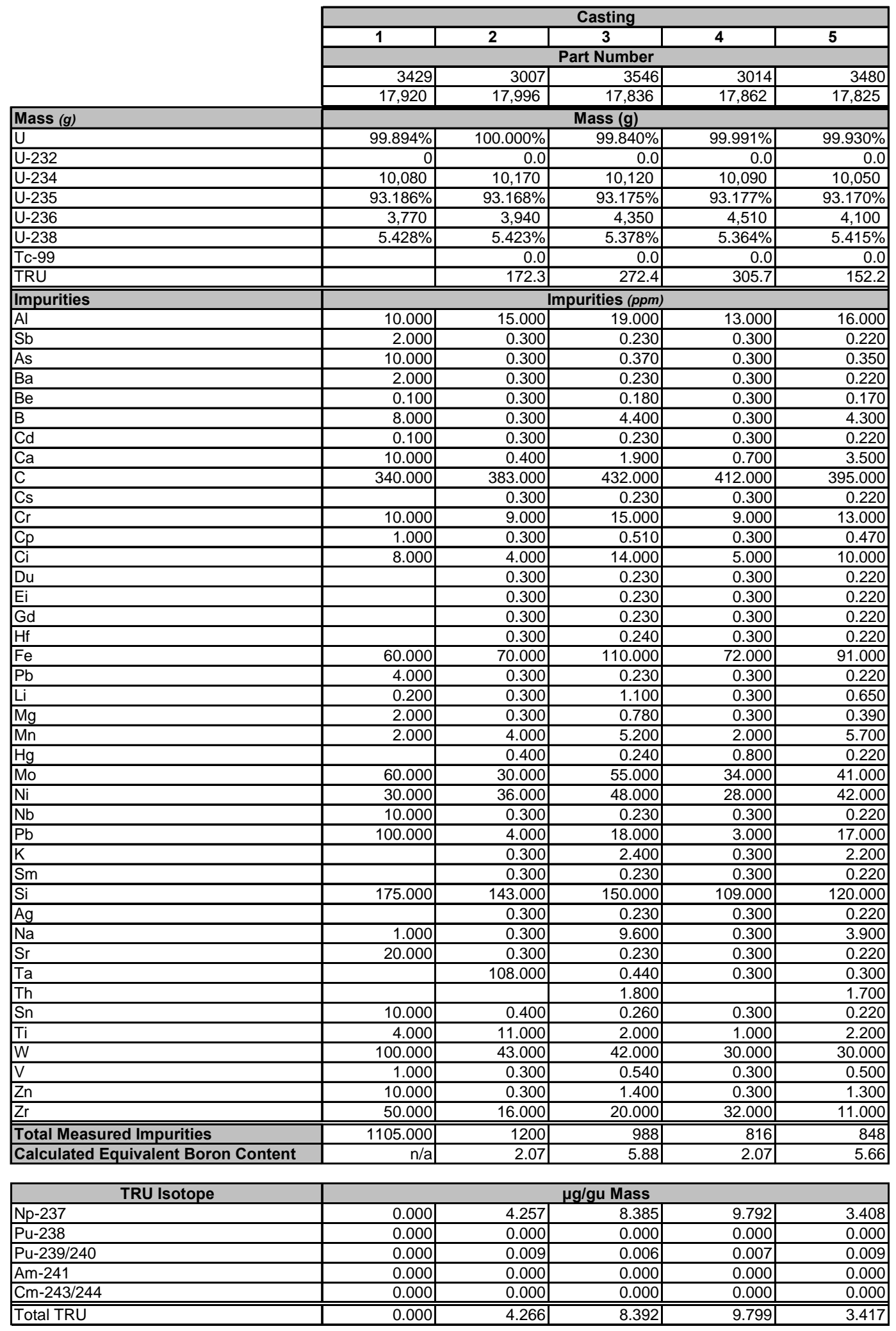


APPENDIX B: DESCRIPTIONS OF THE CANS FOR THE CASTINGS 


\section{APPENDIX B: DESCRIPTIONS OF THE CANS FOR THE CASTINGS}

The description of the stainless steel cans is given in the drawings in this appendix.

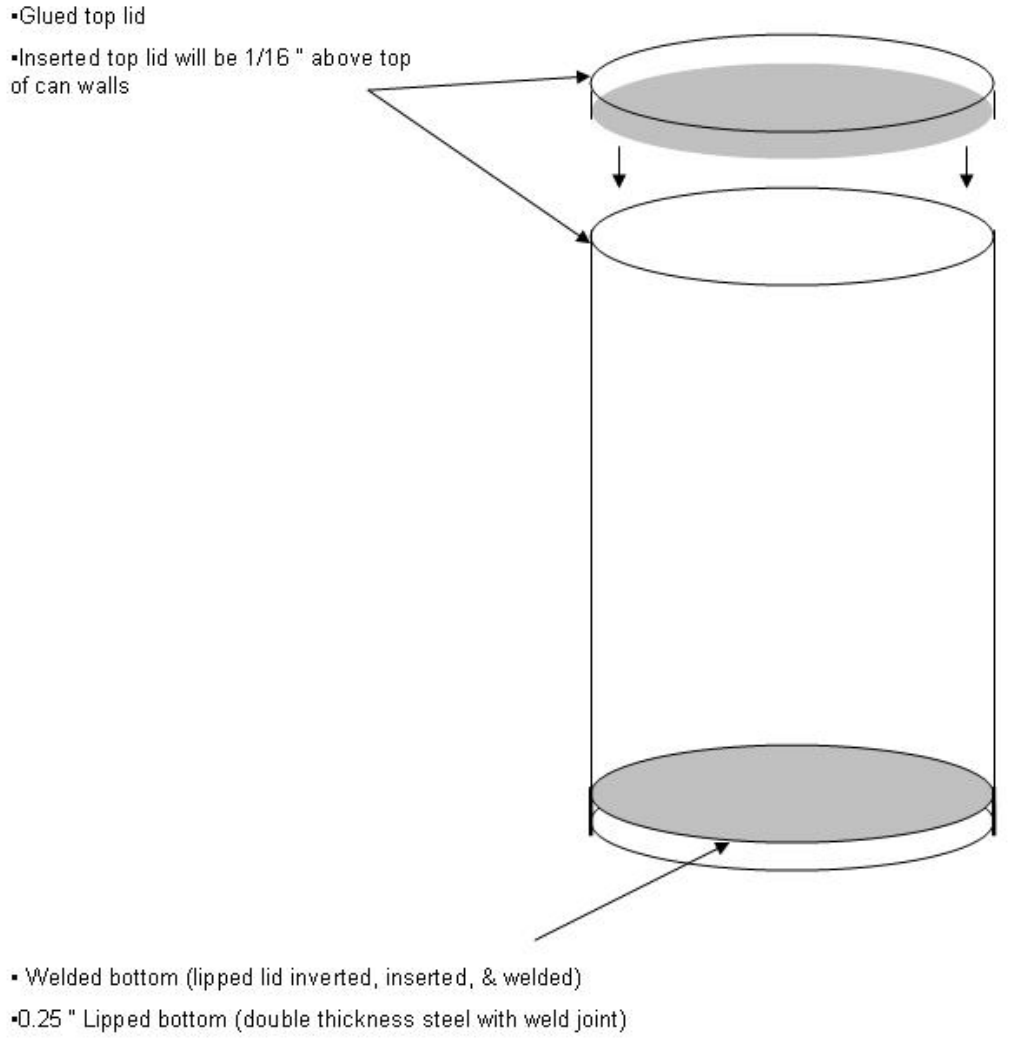

\section{Can type 1 - Cylinder}

Material: $304 \mathrm{~L}$ stainless steel

ID: $5.01 "+.005-.000$

Height: 7.5 " outer height; 7.00 " inner height

the bottom of the can has a welded, lipped

lid that has been inverted). The lid, when

Fig. B.1. Can type 1: Cylinder. 
- Glued top lid

-Inserted top lid will be 1/16" above top of can walls

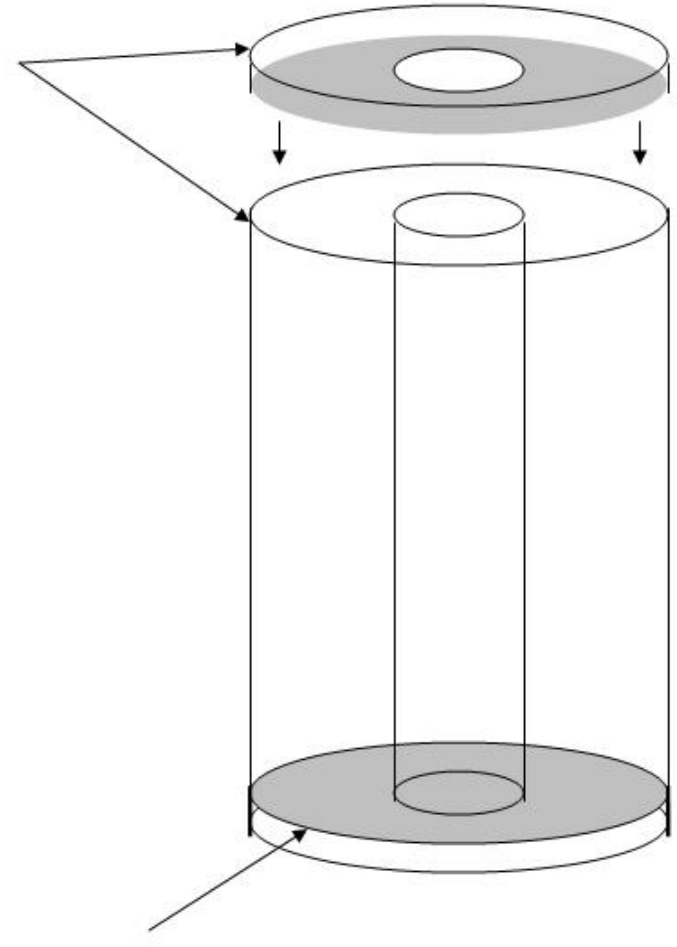

- Welded bottom (lipped lid inverted, inserted, \& welded)

-0.25 " Lipped bottom (double thickness steel with weld joint)
Can type 2 - Cylinder with 3 " inne cylinder (doughnut)

Material: 304L stainless steel

ID: $5.01 "+.005-.000$

Thickness: $025 "$

Height 7.5 " outer height $7.00 "$ " inner

Height 7.5 " outer height, 7.00 " inner

weld ed, lipped lid that has been

inverted). The lid, when pressed, also
inelded, lipped lid that has been

inverted). The lid, when pressed, also
consumes 25 "of the can.

Inner Cylinder: 3" diameter welded at

Inner Cylinder: 3" diameter welded at
bottom; height is flush with sides of outer

cylinder wall

Fig. B.2. Can type 2: Cylinder with 3-in. inner cylinder. 
- Glued top lid

-Inserted top lid will be 1/16"

above top of can walls

- Coated wire handl

\author{
(1)
}
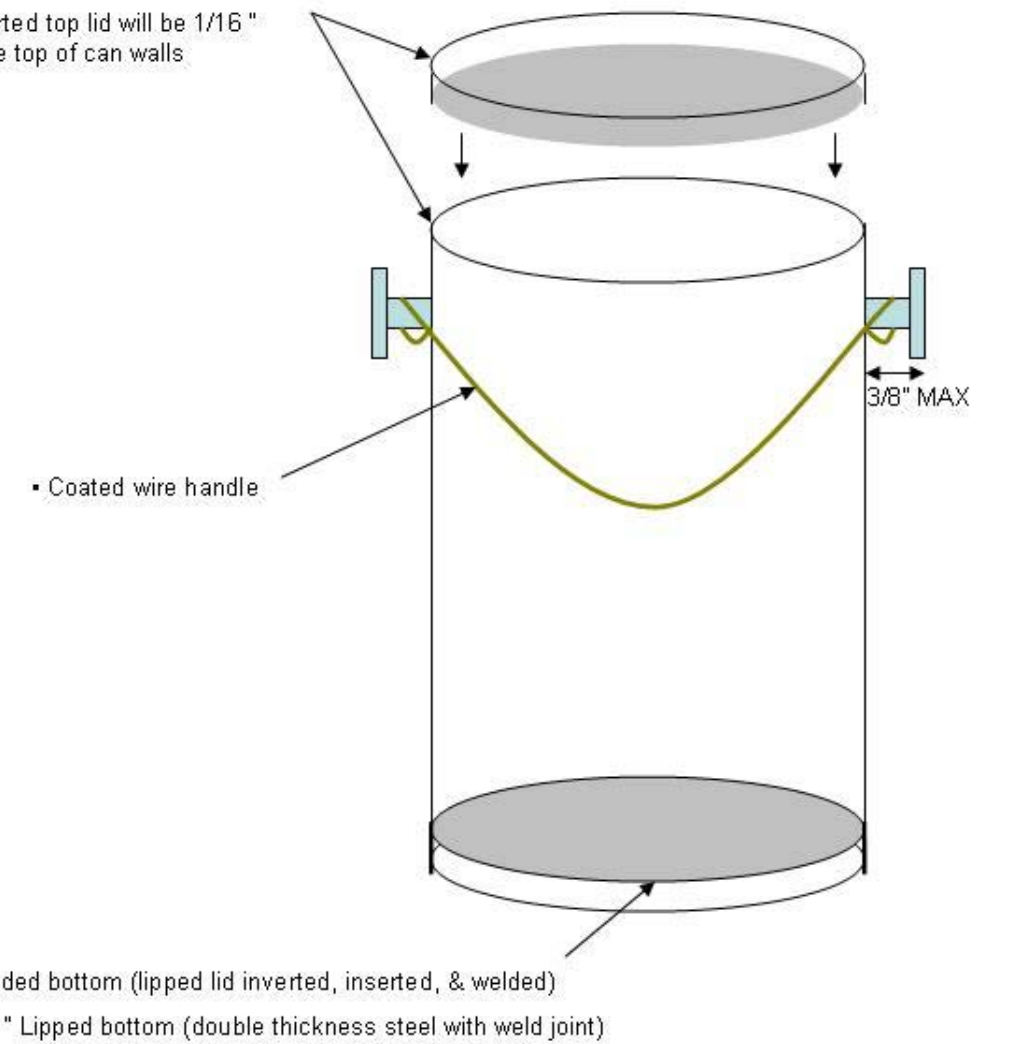

- Welded bottom (lipped lid inverted, inserted, \& welded)

-0.25 " Lipped bottom (double thickness steel with weld joint)
Can type 3- Cylinder with 2 welded trunnions and a cable wire for carrying can

Material: $304 \mathrm{~L}$ stainless stee

D: $5.01 "+.005-.000$

Thickness: $025^{\prime \prime}$

Height: 7.5 " outer height; 7.00 " inner height

(the bottom of the can has a welded, lipped lid

that has been inverted). The lid, when pressed,

also consumes 25 " of the can.

Fig. B.3. Can type 2: Cylinder with two welded trunnions and a cable wire for carrying can. 
APPENDIX C: SOURCE-DETECTOR-CASTING CONFIGURATIONS 


\section{APPENDIX C: SOURCE-DETECTOR-CASTING CONFIGURATIONS}

The source-detector-casting configurations are given in Table C.1. The casting configurations are numbered, but not consecutively. The missing numbers represent measurement configurations that were originally specified for measurement, but represent measurements that were not performed. The identification of the experiments consisted of the number of castings, the configuration number, the type of source, the location for the use of $\mathrm{Cf}$, and the date and a single letter for variations on the run such as a repeat measurement of the same previous identification. The next column in the table gives the number of blocks of data accumulated by the NMIS processor where a block consists of 512 consecutive 1-ns samples that accumulated in approximately a rate of $10^{8}$ in $52 \mathrm{~s}$. The last column of the table contains comment on the particular measurement, such as source correction factor, and other details. The number of blocks in units of $10^{8}$ times $52 \mathrm{~s}$ gives the measurement time in seconds. So if a 40 appears in the column for the number of blocks then the measurement time is $40 \times 52 \mathrm{~s}=34.6 \mathrm{~min}$. 
Table C.1. Measurement descriptions, including source-detector-casting configurations for the measurement

\begin{tabular}{|c|c|c|c|c|c|}
\hline \# & Diagram & Filename & $\begin{array}{l}\text { Blocks } \\
\mathrm{x} 10^{8}\end{array}$ & Page & Notes \\
\hline \multirow[t]{4}{*}{1} & & 1log_cfin_060807a & 10 & 31 & $\begin{array}{l}\text { Cf source } 3.25 \text { in above table. Parallel plates of Cf chamber } \\
\text { are horizontal. Boards linked }\end{array}$ \\
\hline & & 1log_cfin_060808b & 50 & 31 & $\begin{array}{l}\text { Pulse width changed. } 70 \mathrm{nsec} \text { on } \mathrm{Cf} ; 30 \mathrm{nsec} \text { on detectors } \\
\text { Boards linked }\end{array}$ \\
\hline & & $\begin{array}{l}\text { 1log_1_cfin_060825 } \\
\text { 1log_1_cfin_060825a }\end{array}$ & $\begin{array}{l}40 \\
5-1024\end{array}$ & $\begin{array}{l}69 \\
69\end{array}$ & \\
\hline & & 1log_1_cfin_060829 & $30-1024$ & 75 & \\
\hline \multirow[t]{3}{*}{2} & & $\begin{array}{l}1 \log _{\text {_cfout_0 } 060807 a} \\
1 \log _{\text {_cffout_060807b }}\end{array}$ & $\begin{array}{l}50 \\
50\end{array}$ & $\begin{array}{l}31 \\
31\end{array}$ & $\begin{array}{l}\text { Det } 4 \text { not near the casting but at the edge of the table } \\
\text { Det } 4 \text { in multiplicity by mistake in run a but fixed in run b. Cf } \\
\text { plates horizontal }\end{array}$ \\
\hline & & 1log_cfout_060608a & 50 & 33 & $\begin{array}{l}\text { Cf Pulse width changed from } 50 \text { to } 70 \text { nsec. Reset the } \mathrm{Cf} \\
\text { CFD to } 110 \text { after PHS curve }\end{array}$ \\
\hline & & 1log_cfout_060808b & 40 loops & 33 & New NMIS software \\
\hline 3 & & $\begin{array}{l}1 \log \_\mathrm{dt} \_060808 \\
1 \operatorname{logs} \_d t \_060825 \\
1 \log 33 \text { dt_060829 }\end{array}$ & $\begin{array}{l}40 \text { loops } \\
40 \text { loops } \\
30-1024\end{array}$ & $\begin{array}{l}35 \\
68 \\
75\end{array}$ & $\begin{array}{l}\text { Dt gen } 3 \text { in. above table } \\
\text { Source correction factor was } 1.01 \text { instead of } 1.03 \\
\text { Det } 4 \text { is } 15 \text { in.from beam spot against DT pipe on det } 1 \text { side. } \\
\text { Circular aperture } \\
\text { Det } 4 \text { is } 15 \text { in. from the beam spot against DT pipe on det } 1 \\
\text { side, circular aperture, circular aperture }\end{array}$ \\
\hline
\end{tabular}


Table C.1. Measurement descriptions, including source-detector-casting configurations for the measurement

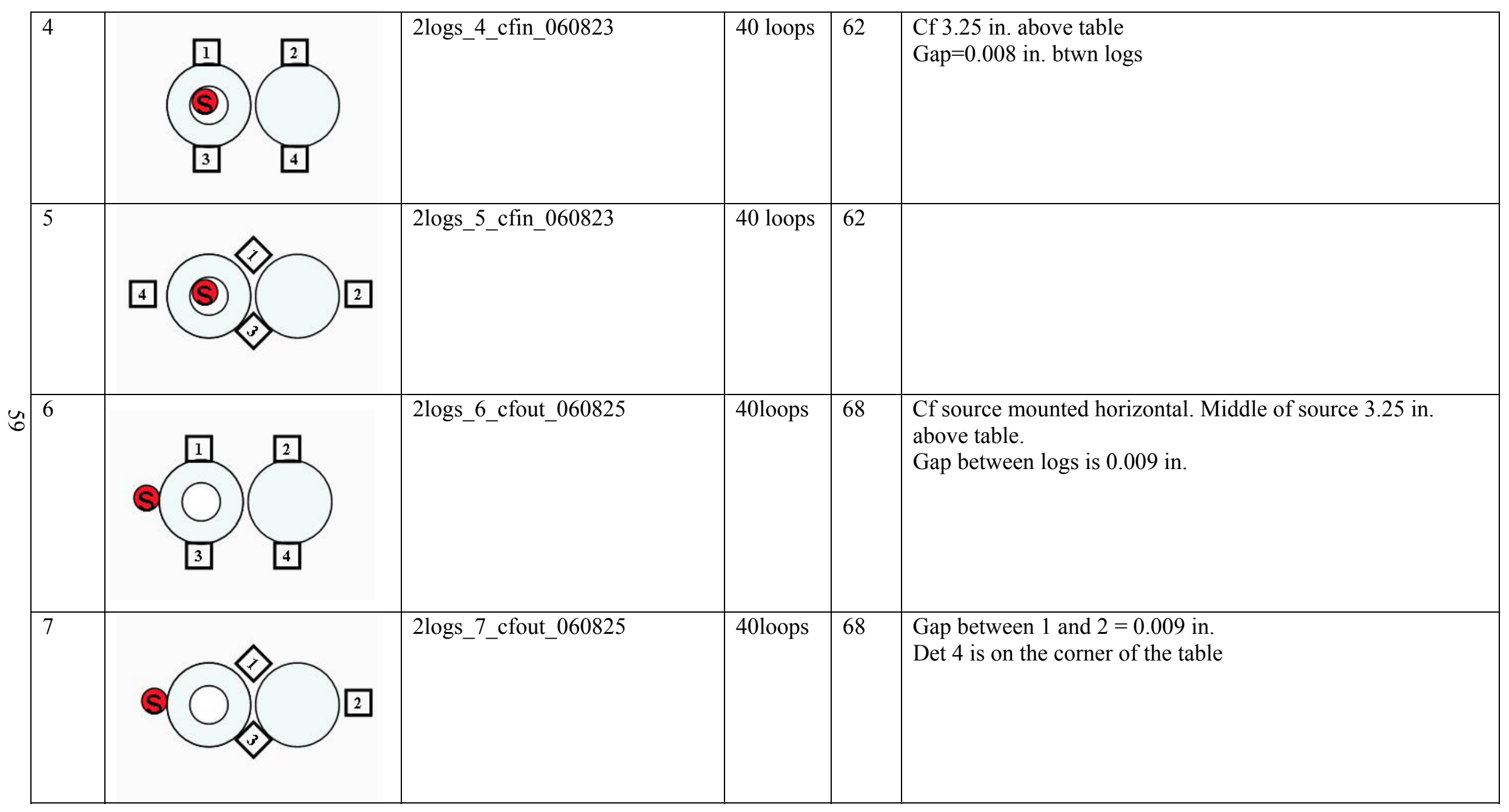


Table C.1. Measurement descriptions, including source-detector-casting configurations for the measurement

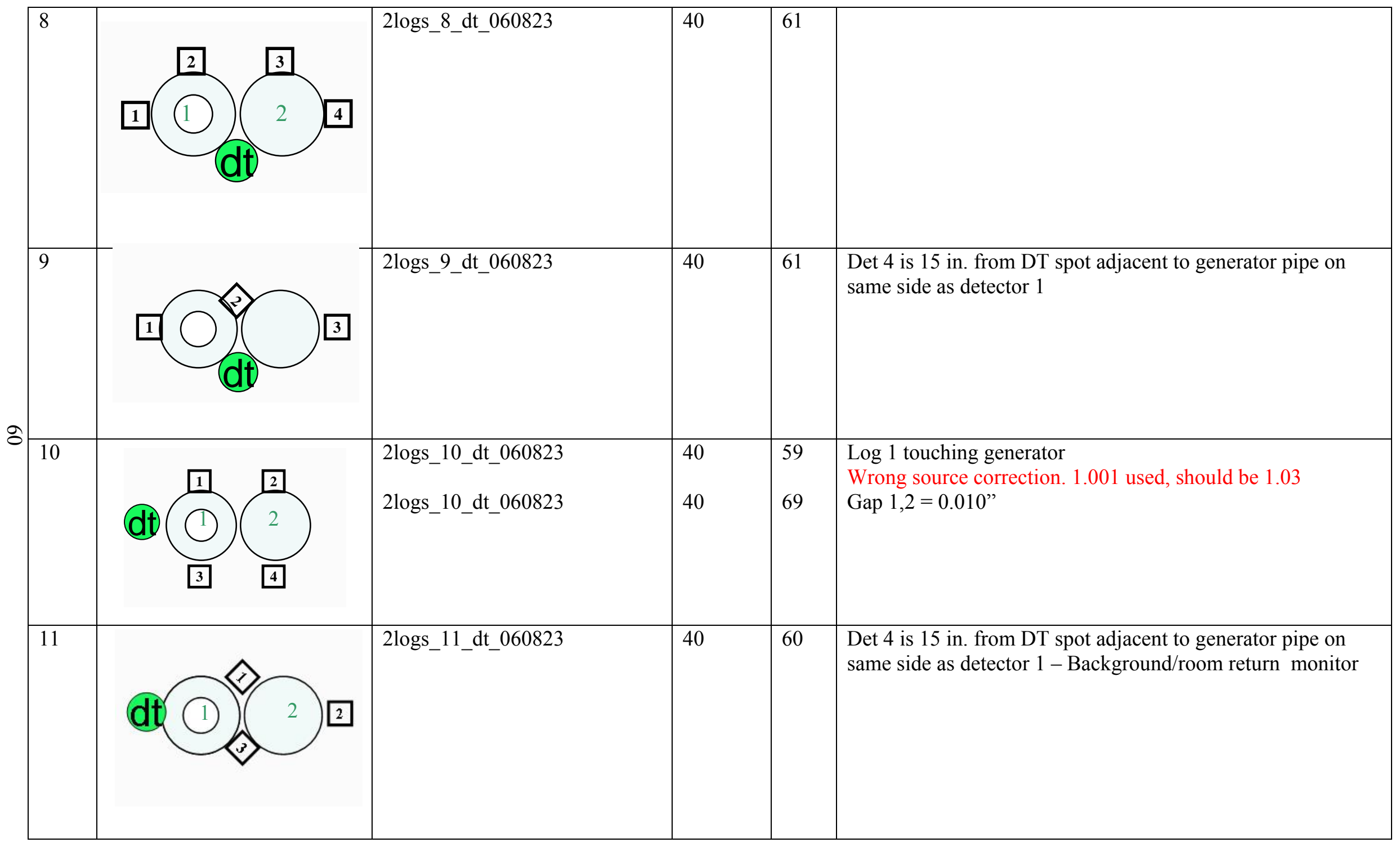


Table C.1. Measurement descriptions, including source-detector-casting configurations for the measurement

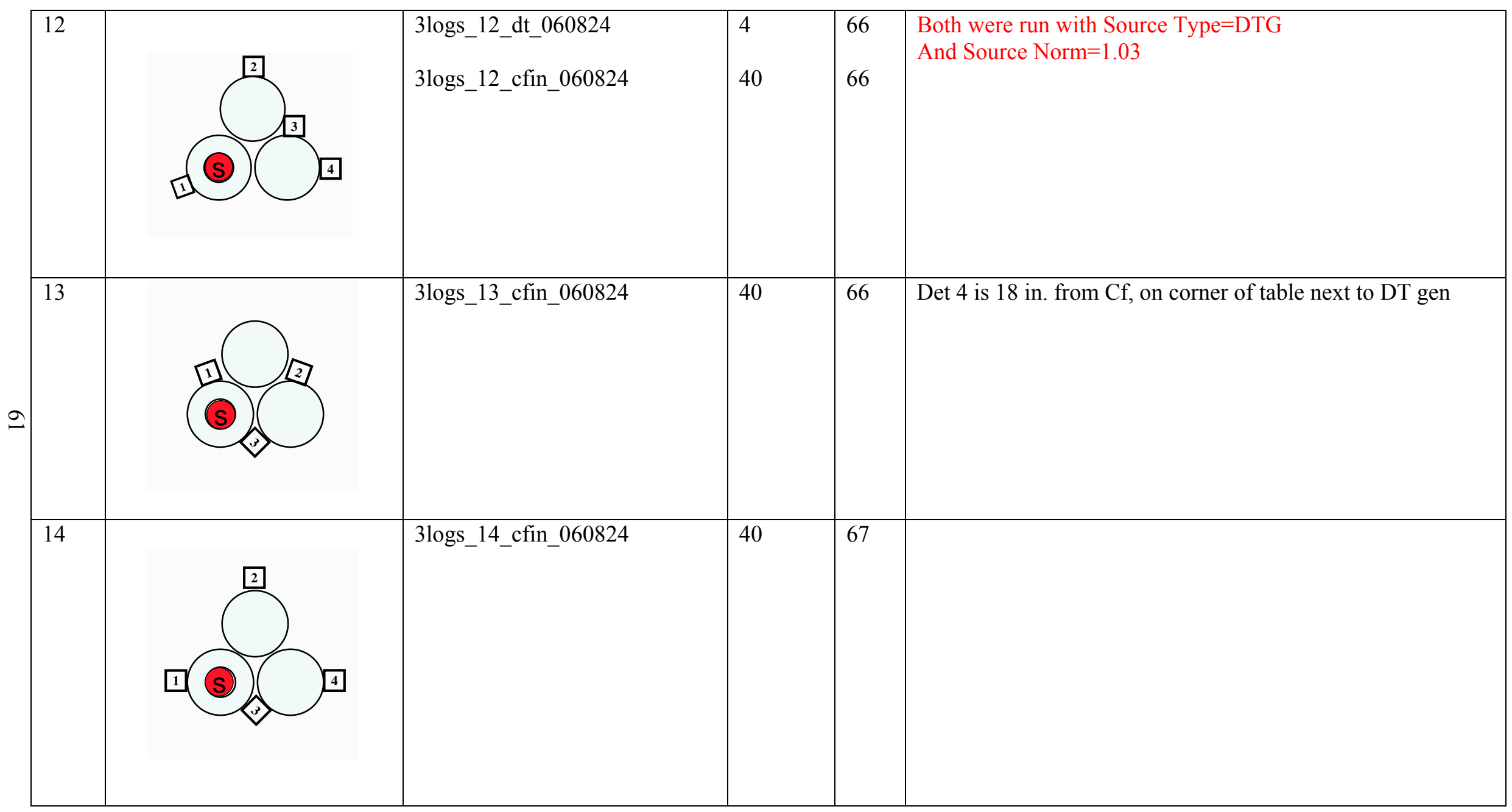


Table C.1. Measurement descriptions, including source-detector-casting configurations for the measurement

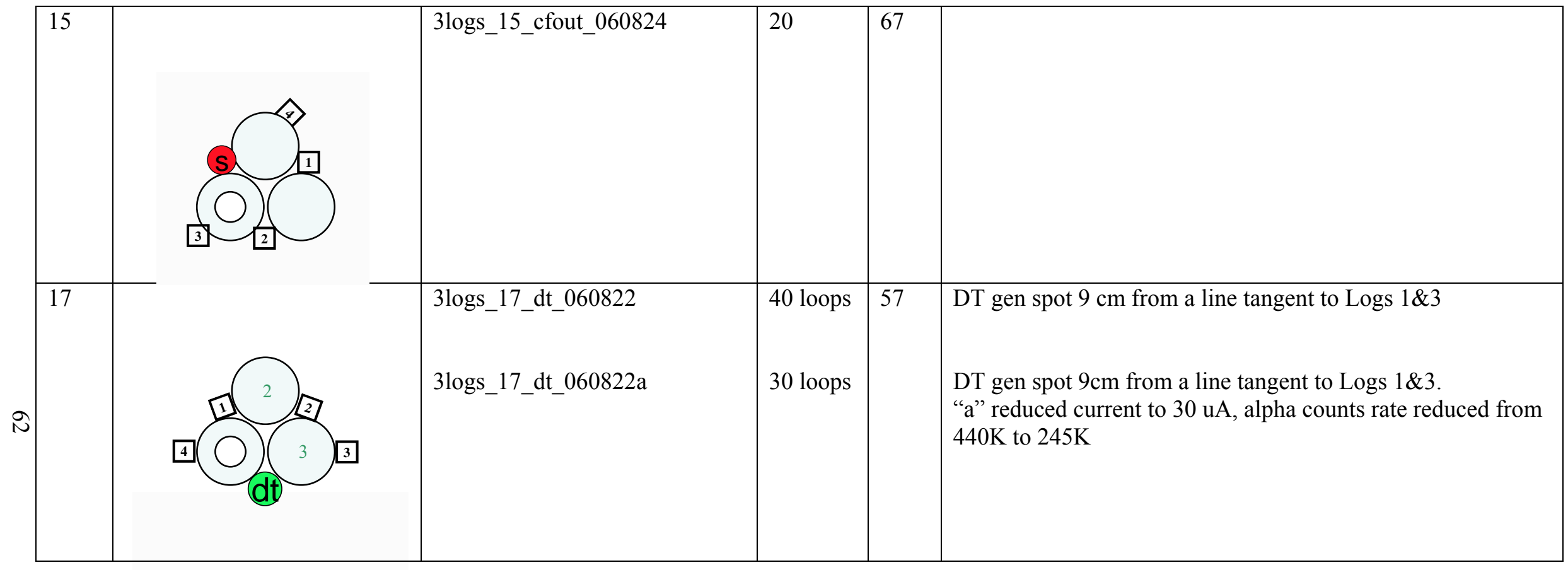


Table C.1. Measurement descriptions, including source-detector-casting configurations for the measurement

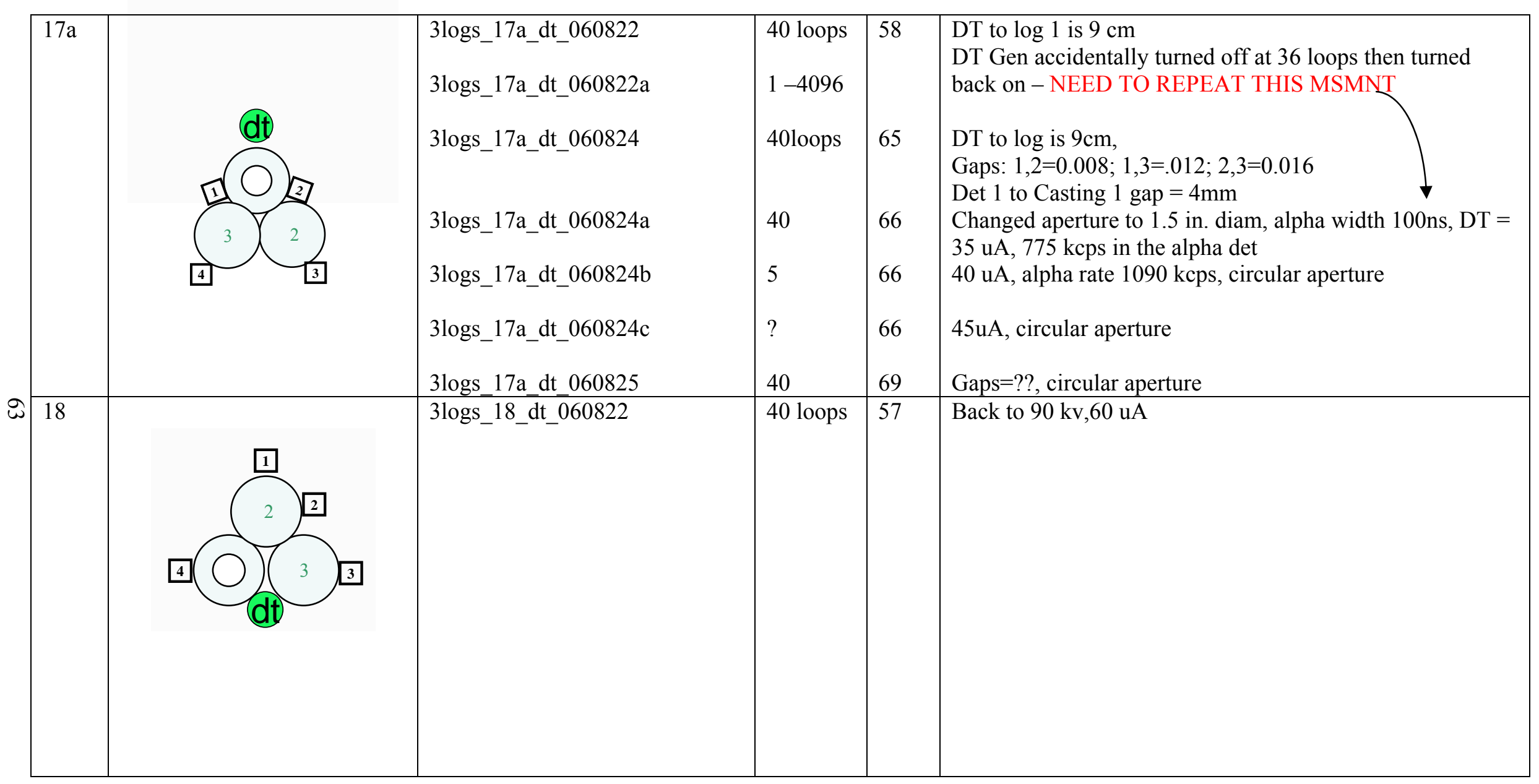


Table C.1. Measurement descriptions, including source-detector-casting configurations for the measurement

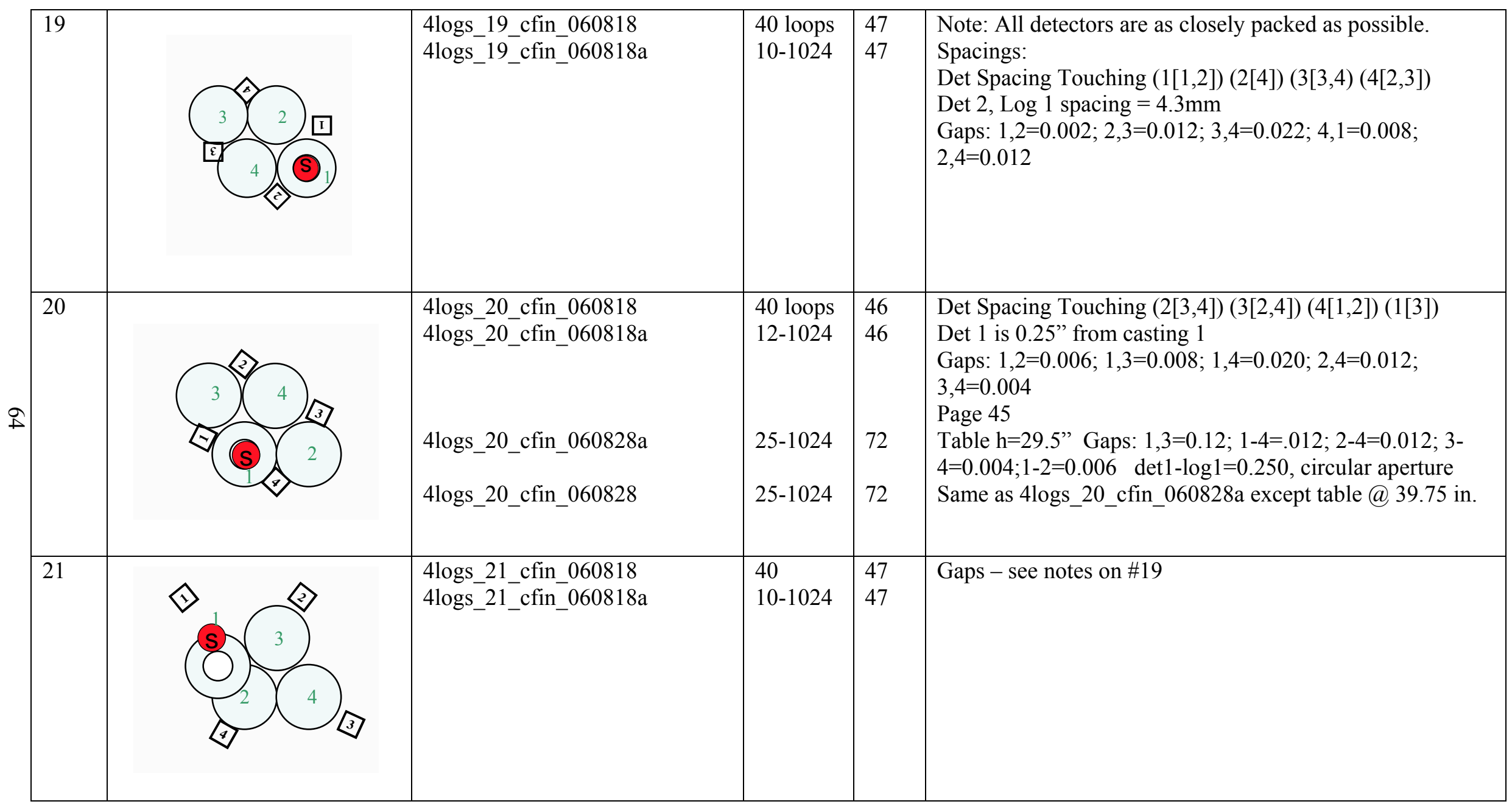


Table C.1. Measurement descriptions, including source-detector-casting configurations for the measurement

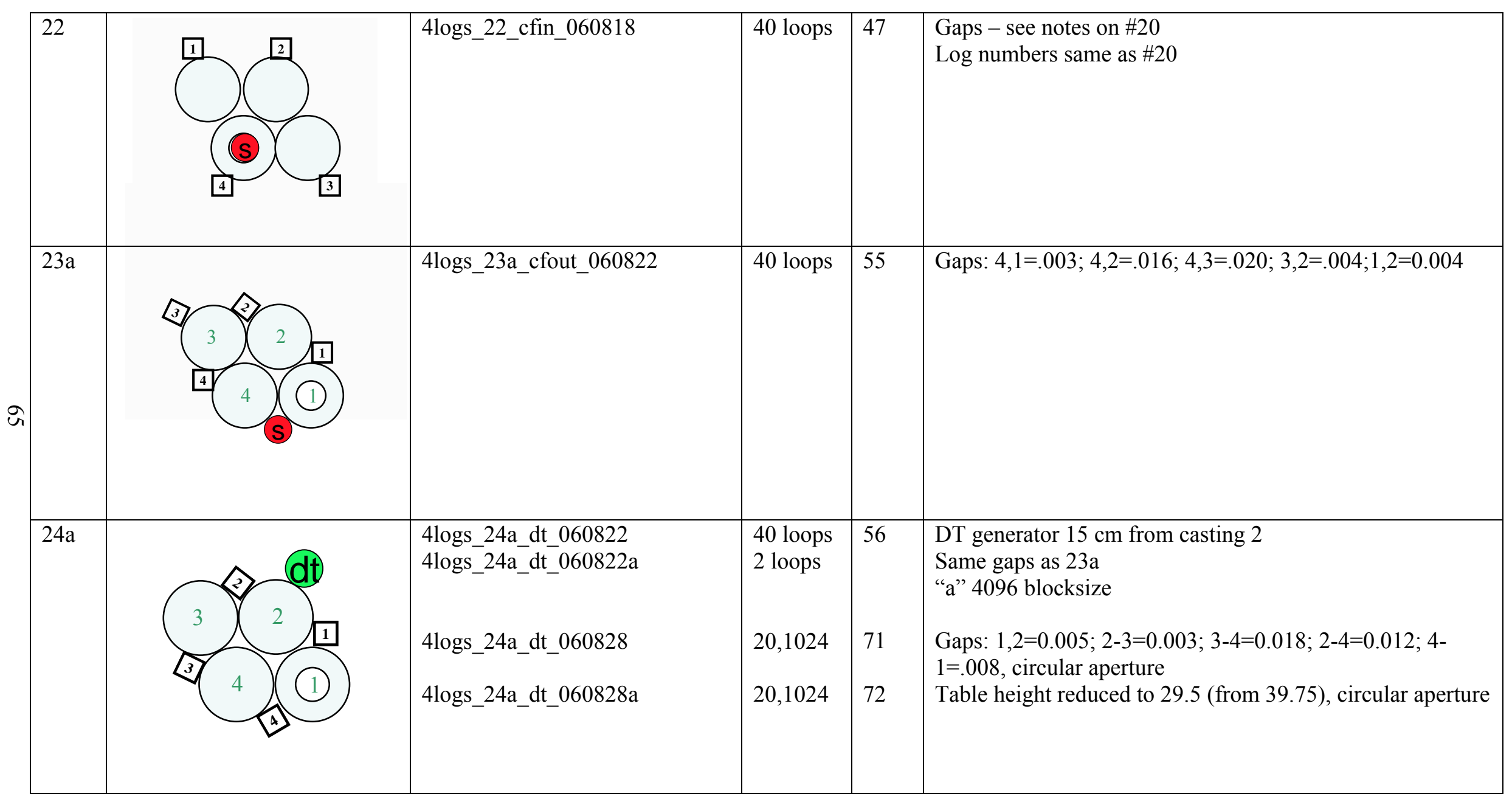


Table C.1. Measurement descriptions, including source-detector-casting configurations for the measurement

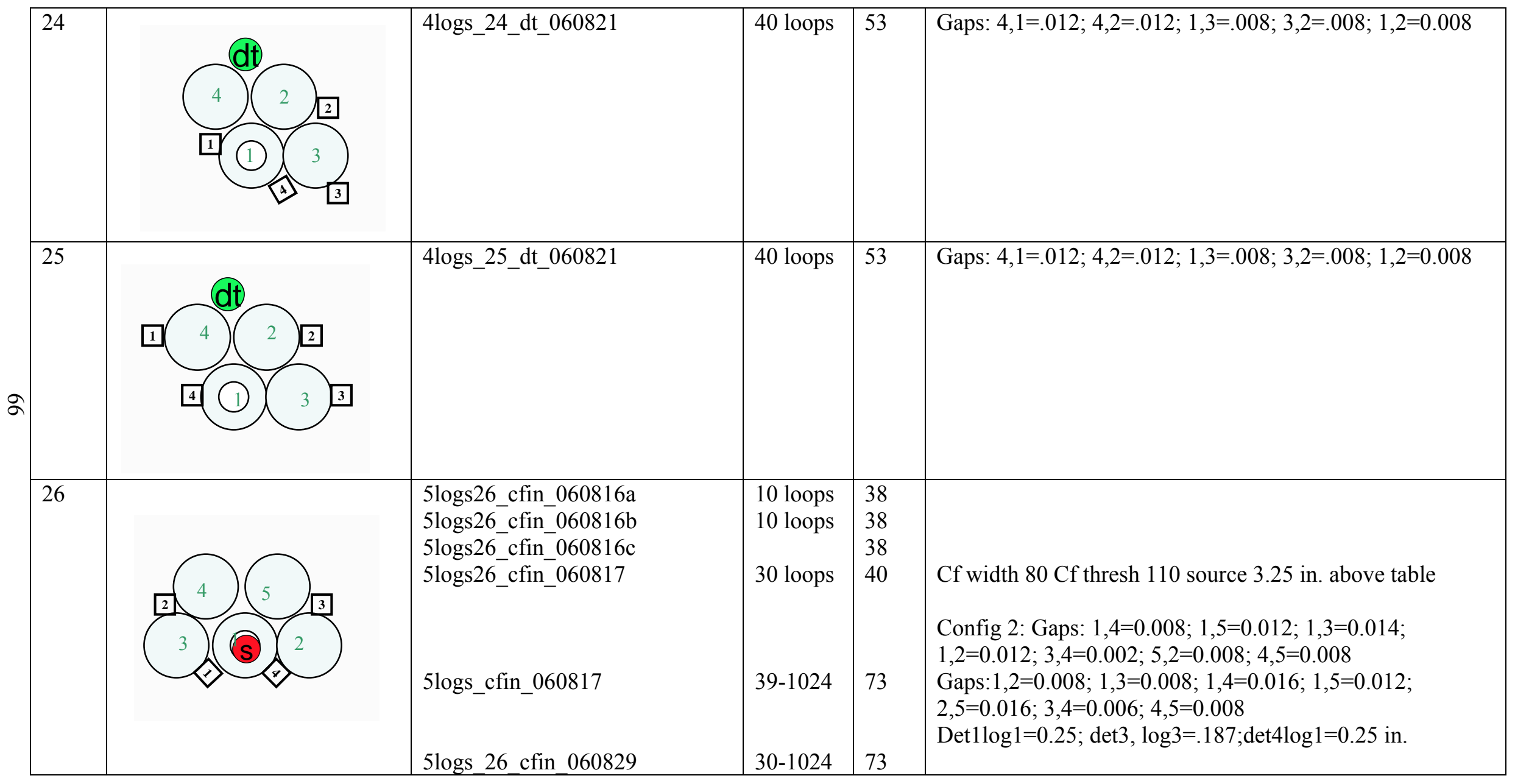


Table C.1. Measurement descriptions, including source-detector-casting configurations for the measurement

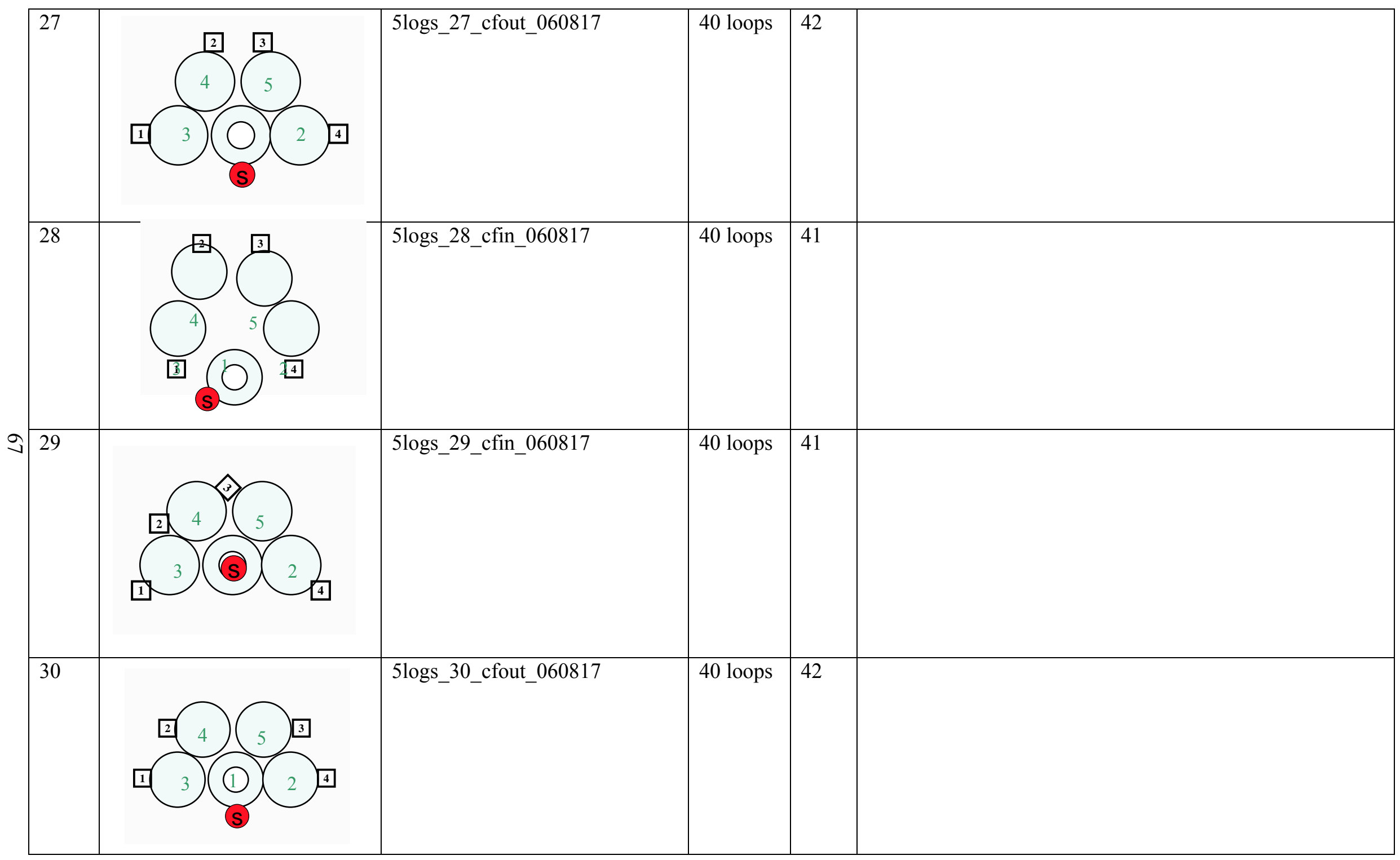


Table C.1. Measurement descriptions, including source-detector-casting configurations for the measurement

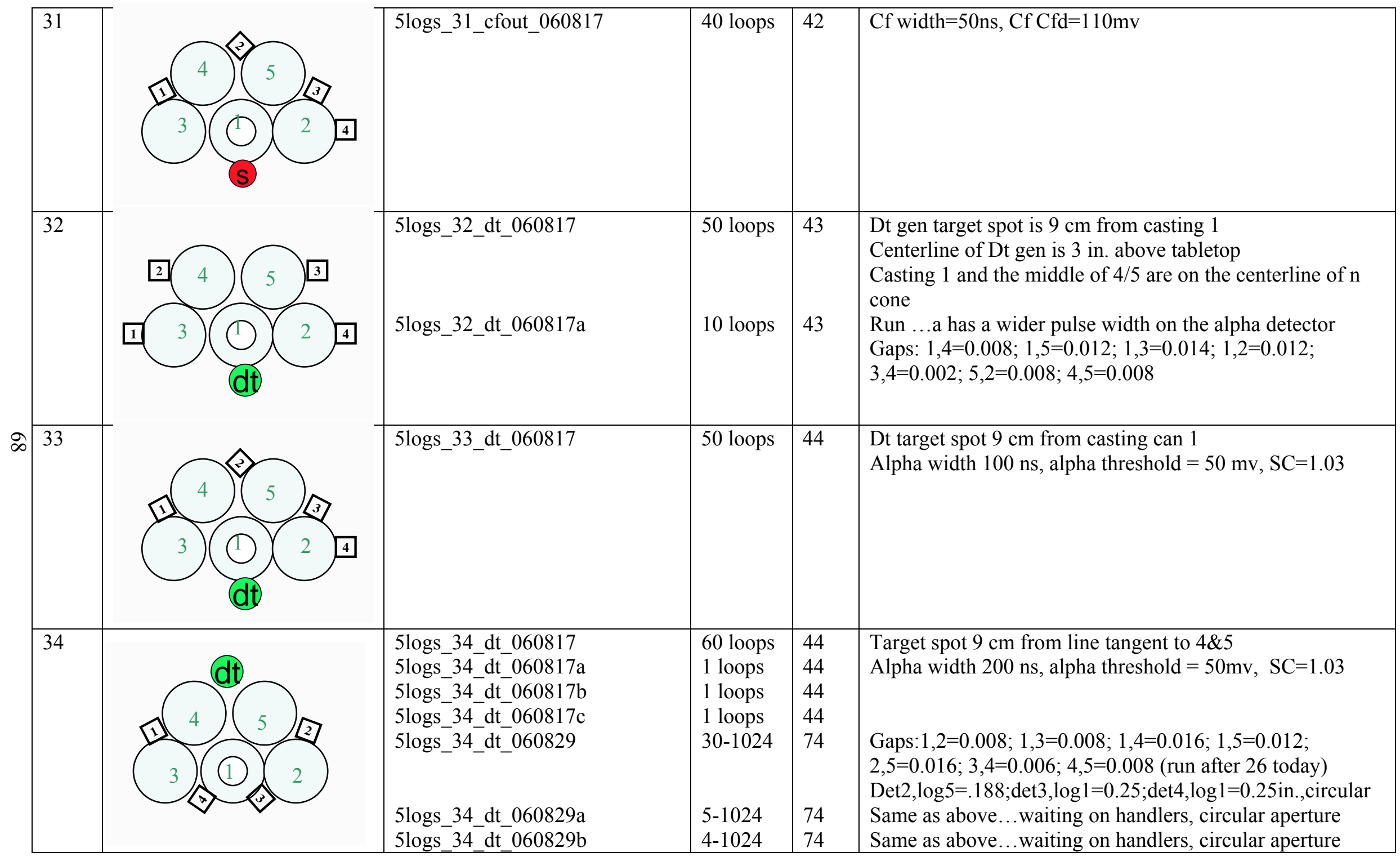


Table C.1. Measurement descriptions, including source-detector-casting configurations for the measurement

\begin{tabular}{|c|c|c|c|c|c|}
\hline 0 & $\begin{array}{l}\text { No logs on table, dets with Cf } \\
\text { only }\end{array}$ & $\begin{array}{l}\text { 0logs_0_cf_060821 } \\
\text { 0logs_0_cf_060831 } \\
\text { 0logs_0_cf_060831a }\end{array}$ & $\begin{array}{l}40 \\
60,1024 \\
60,1024 \\
\end{array}$ & $\begin{array}{l}52 \\
81 \\
81\end{array}$ & $\begin{array}{l}\text { See diagram for placement of detectors } \\
\text { Table@ } 39.75 \text { in. } \\
\text { Table@ } 29.5 \text { in. }\end{array}$ \\
\hline 0 & $\begin{array}{l}\text { No logs on table, dets with Dt } \\
\text { only }\end{array}$ & $\begin{array}{l}0 \log s \_0 \_d t \_060821 \\
010 g s \_0 \_d t \_060829\end{array}$ & $\begin{array}{l}40 \\
60,1024\end{array}$ & $\begin{array}{l}52 \\
79\end{array}$ & $\begin{array}{l}\text { See diagram for placement of detectors } \\
\text { Power glitch?? DT failed, finally back up, but alpha rate } \\
645 \mathrm{~K} \text { at } 90 \mathrm{kV}, 35 \mathrm{uA} \text {. Was } \sim 725 \mathrm{~K} \text {. }\end{array}$ \\
\hline
\end{tabular}

Notes:

Convention on spacing for detector to casting: (detector[casting, casting])

Convention for casting gaps: casting\#,casting\#=gap ; casting\#,casting\#=gap (where gap is in inches unless noted otherwise)

Convention block size is 512 unless otherwise noted

Casting Numbers (copy and paste):

Other notes:

oे $\quad 0824061050 \mathrm{am}$

o Change aperture in DT generator from $11 \mathrm{~mm} \times 2.5 \mathrm{in}$. slit to $1.5 \mathrm{in}$. diam. slit

o Changed alpha pulse width to $100 \mathrm{~ns}$

o Decreased DT generator current to $35 \mathrm{uA}$

- Alpha count rate now $775 \mathrm{kcps}$

o Now run 17a again -> 3logs_17a_dt_060824a

- 082506

o Staring with the $\mathrm{Cf}$ out run number 6

o Changed width on the alpha pulse form the DT generator to $200 \mathrm{~ns}$ (start of the day)

0 We do notice that the table moves a little each time material is placed on the table, which means that the cone of the beam may not always be perfectly aligned. Today, we noticed it in the data, made corrections and restarted the run.

- 082806

o Table height is 39.75 unless otherwise noted

- 082906

o Did MCA runs to look at alpha detector 
APPENDIX D: GAPS BETWEEN CASTINGS CANS FOR VARIOUS SOURCE-DETECTOR-CASTING CONFIGURATIONS 


\section{APPENDIX D: GAPS BETWEEN CASTINGS CANS FOR VARIOUS SOURCE-DETECTOR-CASTING CONFIGURATIONS}

The gaps between castings for various configurations were measure with a convention feeler gauge and are given in Table D.1. These gaps can be included in Monte Carlo calculations for these measurements. These gaps were measured after the excess glue had been removed from the lids of the cans. The relative orientation of the casting in an array was maintained for all configurations. That is, the rotational orientation of cans 1 and 2 were the same whether these two cans were adjacent for a two casting can configuration or a four casting can configuration.

Table D.1. Gaps between castings cans for various source-detector-casting configurations (Gap between casting is $10^{-3}$ in.)

\begin{tabular}{ccccccccccc}
\hline $\begin{array}{c}\text { Number of } \\
\text { cans and } \\
\text { configuration }\end{array}$ & $\begin{array}{c}\text { Page } \\
\text { number in } \\
\text { log book }\end{array}$ & $\mathbf{1 - 2}$ & $\mathbf{1 - 3}$ & $\mathbf{1 - 4}$ & $\mathbf{1 - 5}$ & $\mathbf{2 - 3}$ & $\mathbf{2 - 4}$ & $\mathbf{2 - 5}$ & $\mathbf{3 - 4}$ & $\mathbf{4 - 5}$ \\
\hline $3-17$ & 58 & 10 & 6 & - & - & 18 & - & - & - & - \\
$3-17$ & 64 & 12 & 1 & - & - & 12 & - & - & - & - \\
$3-17$ & 70 & 11 & 27 & - & - & 27 & - & - & - & - \\
$4-19$ & 47 & 2 & - & 8 & - & 12 & 12 & - & 22 & - \\
$4-20$ & 72 & 6 & 12 & 12 & - & - & 12 & - & 4 & - \\
$4-23$ & 55 & 4 & - & 3 & - & 4 & 16 & - & 20 & - \\
$4-24$ & 71 & 5 & - & 8 & - & - & 12 & - & 18 & - \\
$4-25$ & 53 & 8 & 8 & 12 & - & 8 & 12 & - & - & - \\
$5-26$ & 73 & 8 & 8 & 16 & 12 & - & - & 16 & 6 & 8 \\
\hline
\end{tabular}




\section{APPENDIX E: ADDITIONAL COMPARISONS OF MEASUREMENT AND MONTE CARLO CALCULATIONS}




\section{APPENDIX E: ADDITIONAL COMPARISONS OF MEASUREMENT AND MONTE CARLO CALCULATIONS}

This appendix provides supplement plots of comparisons of the MCNP-PoliMi calculations with measured data. Each configuration $(1,3,4,12,20$, and 26) has a plot for each detector showing the full time scale (511 s), a 200-ns time scale, and a $30 \mathrm{~ns}$ time scale. Three plots are provided per detector to highlight the overall fit, the decay slope, and the initial transients, respectively. Also, the cross-correlation graph between a set of detectors is provided for each configuration.

\section{Configuration 1:}

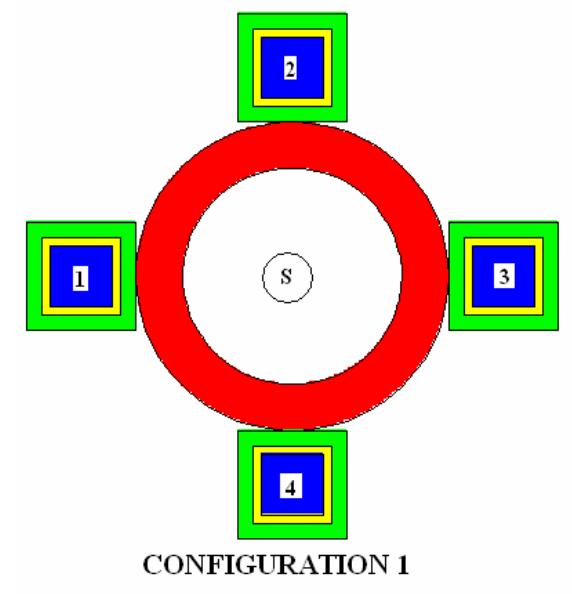

Fig. E.1. Sketch of the calculational model. 


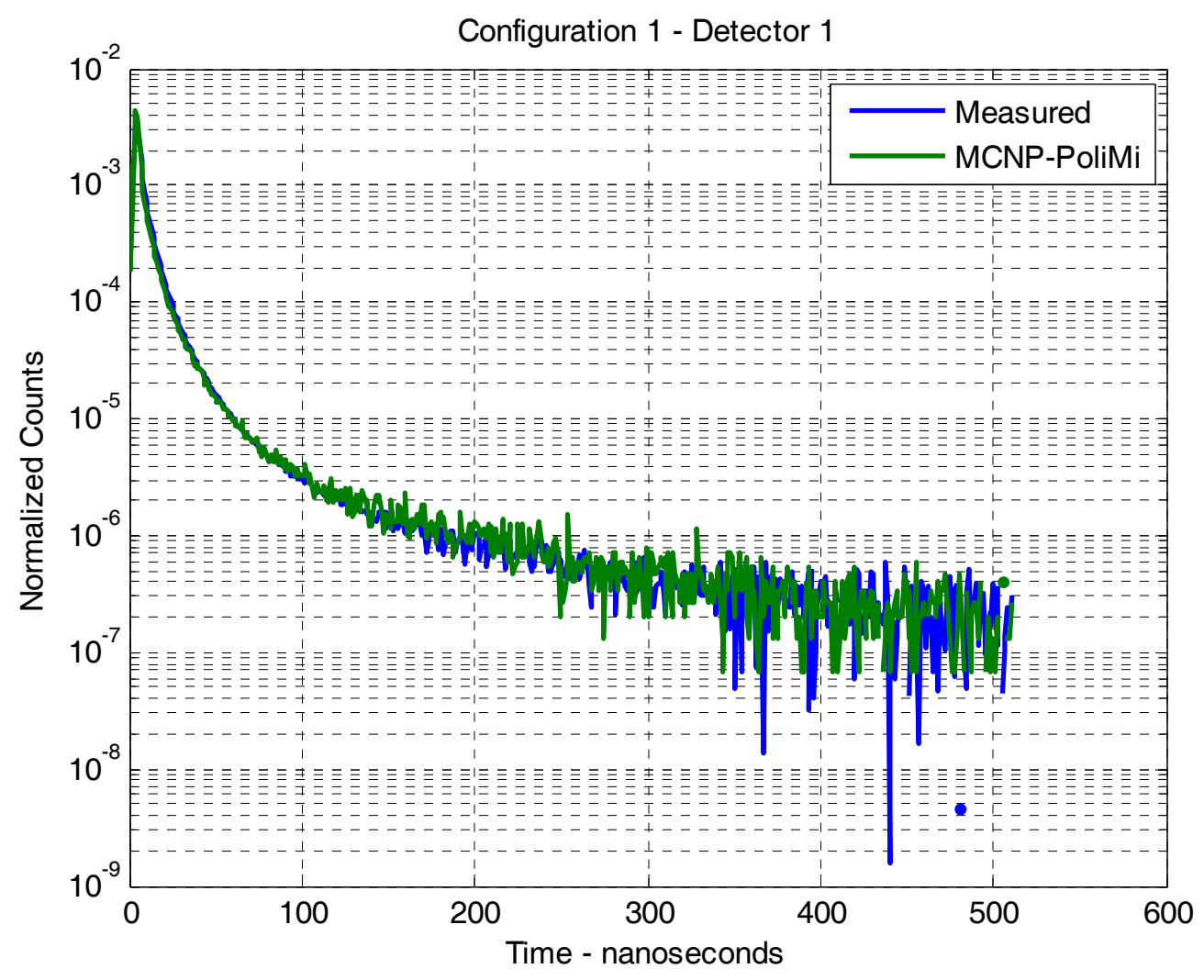

Fig. E.2. Time distribution of counts in detector 1 after $\mathbf{C f}$ fission for 512 ns for configuration 1. 


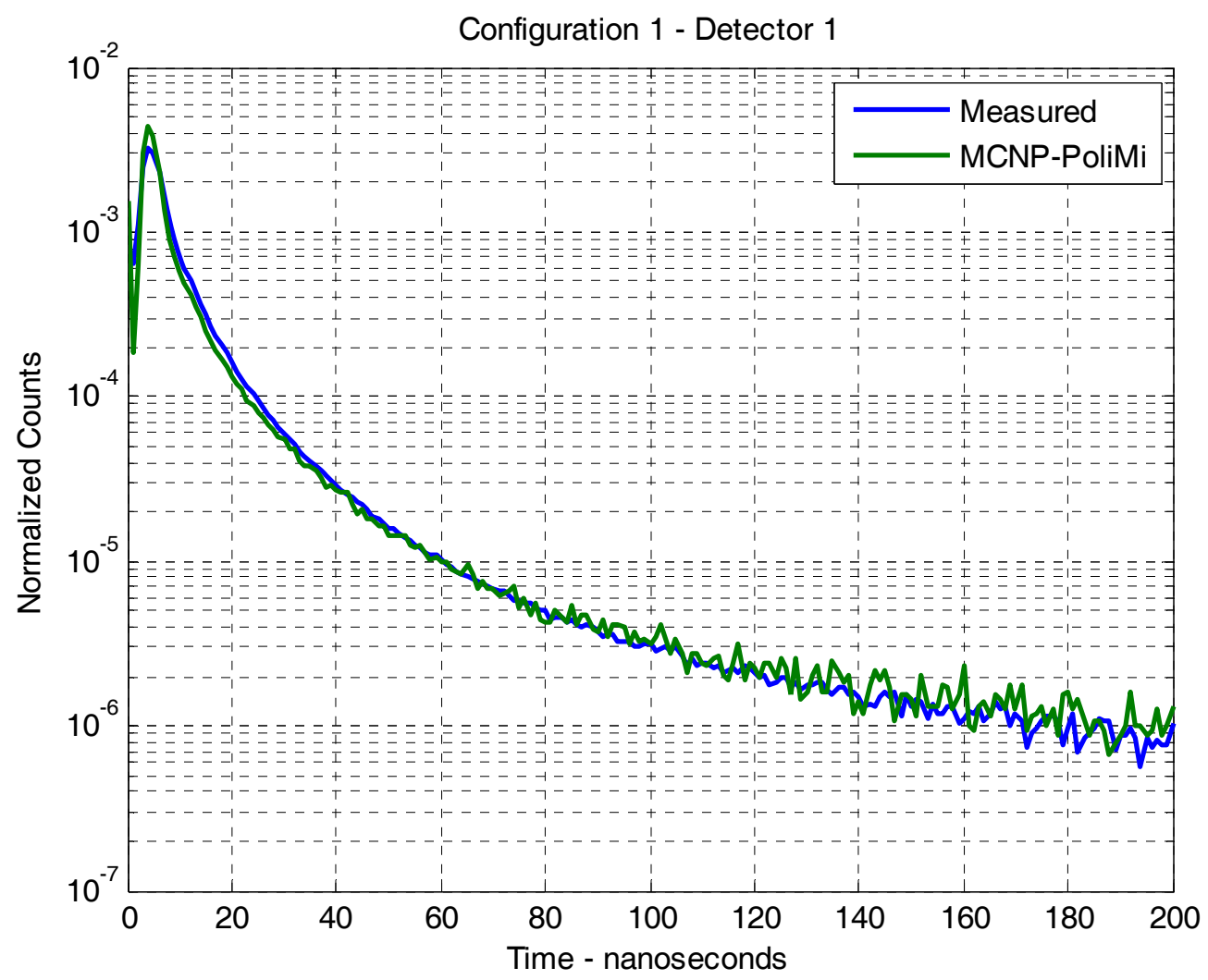

Fig. E.3. Time distribution of counts in detector $\mathbf{1}$ for after $\mathbf{C f}$ fission for $200 \mathrm{~ns}$ for configuration 1. 


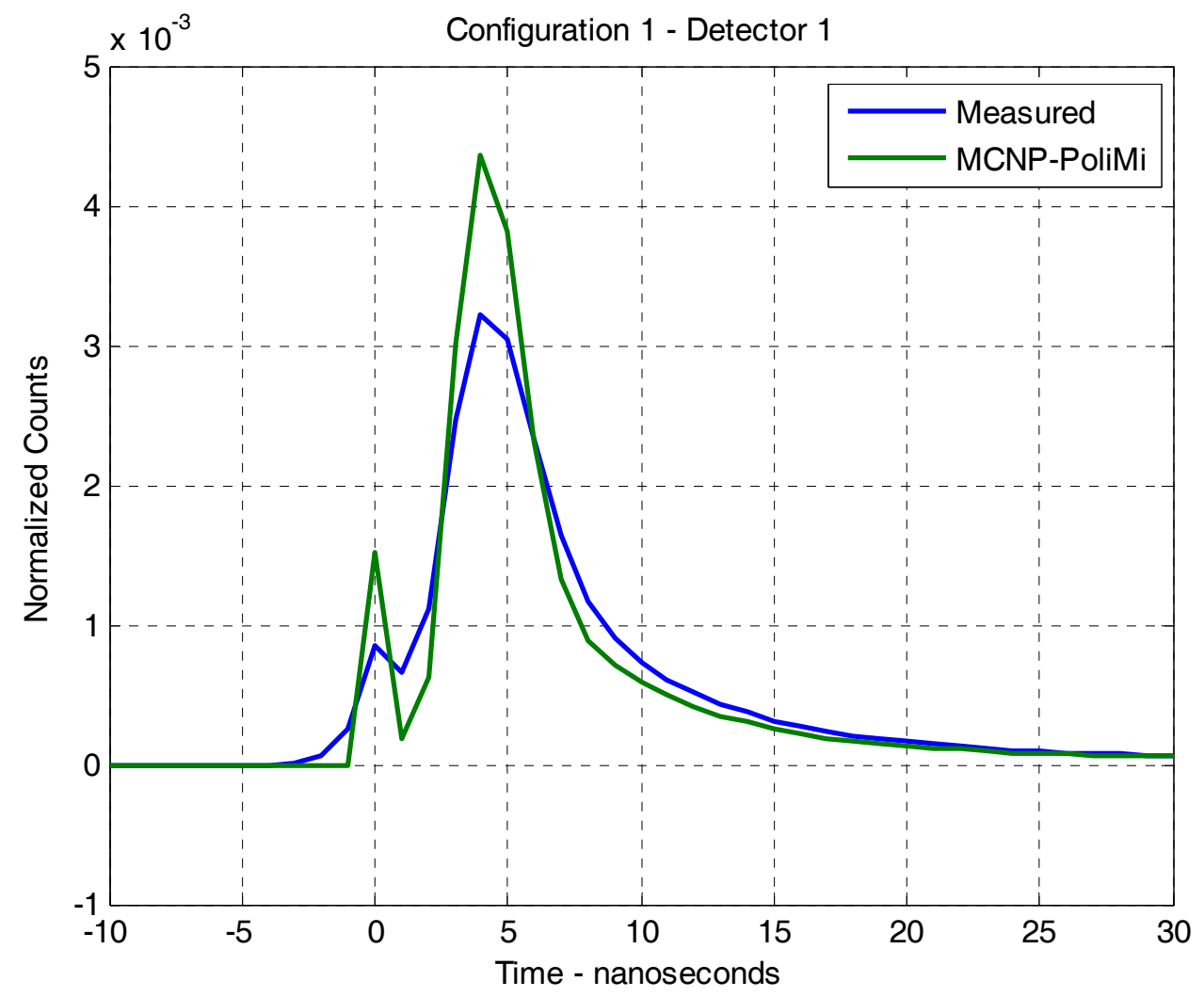

Fig. E.4. Time distribution of counts in detector 1 after $\mathrm{Cf}$ fission for $\mathbf{3 0}$ ns for configuration 1. 


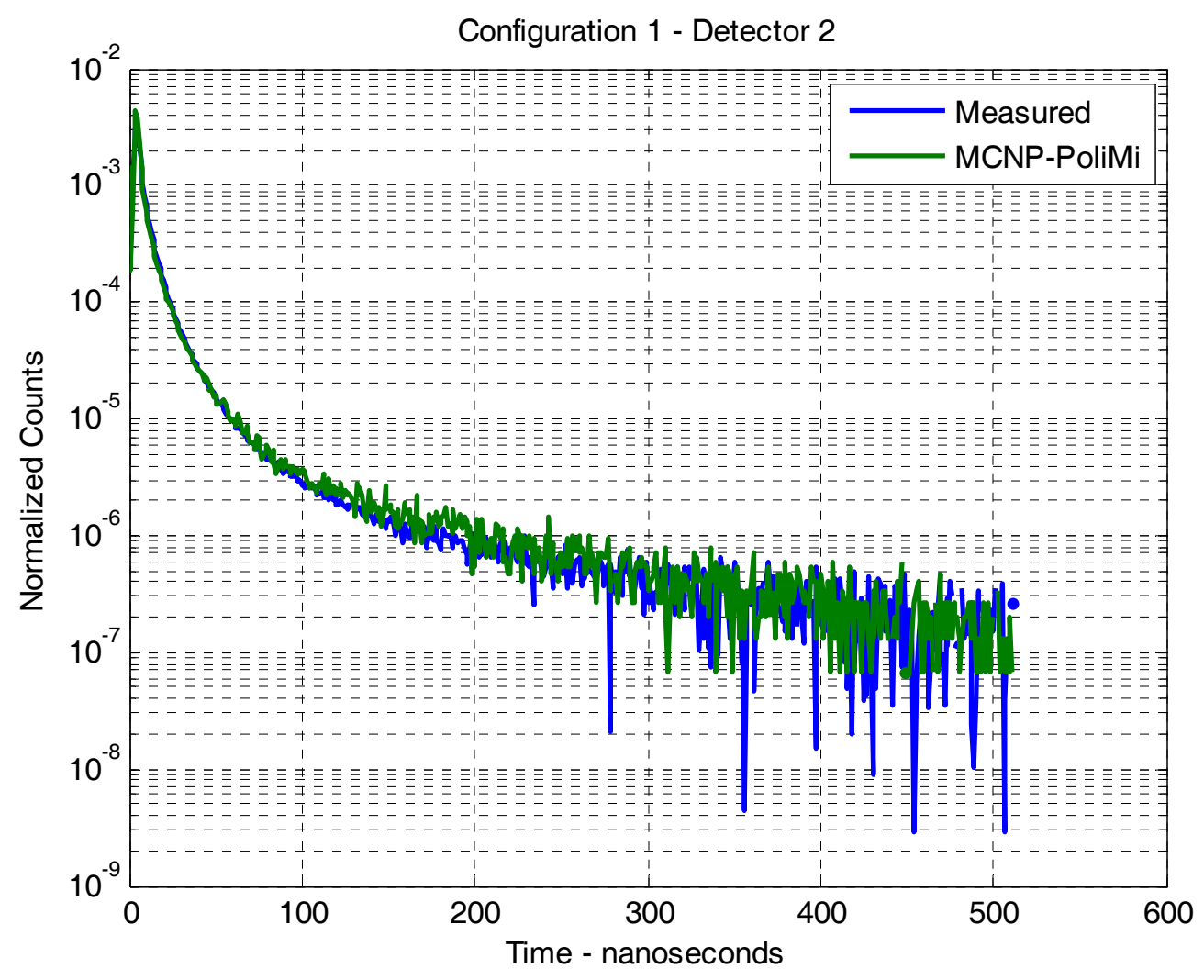

Fig. E.5. Time distribution of counts in detector 2 after $\mathbf{C f}$ fission for 512 ns for configuration 1. 


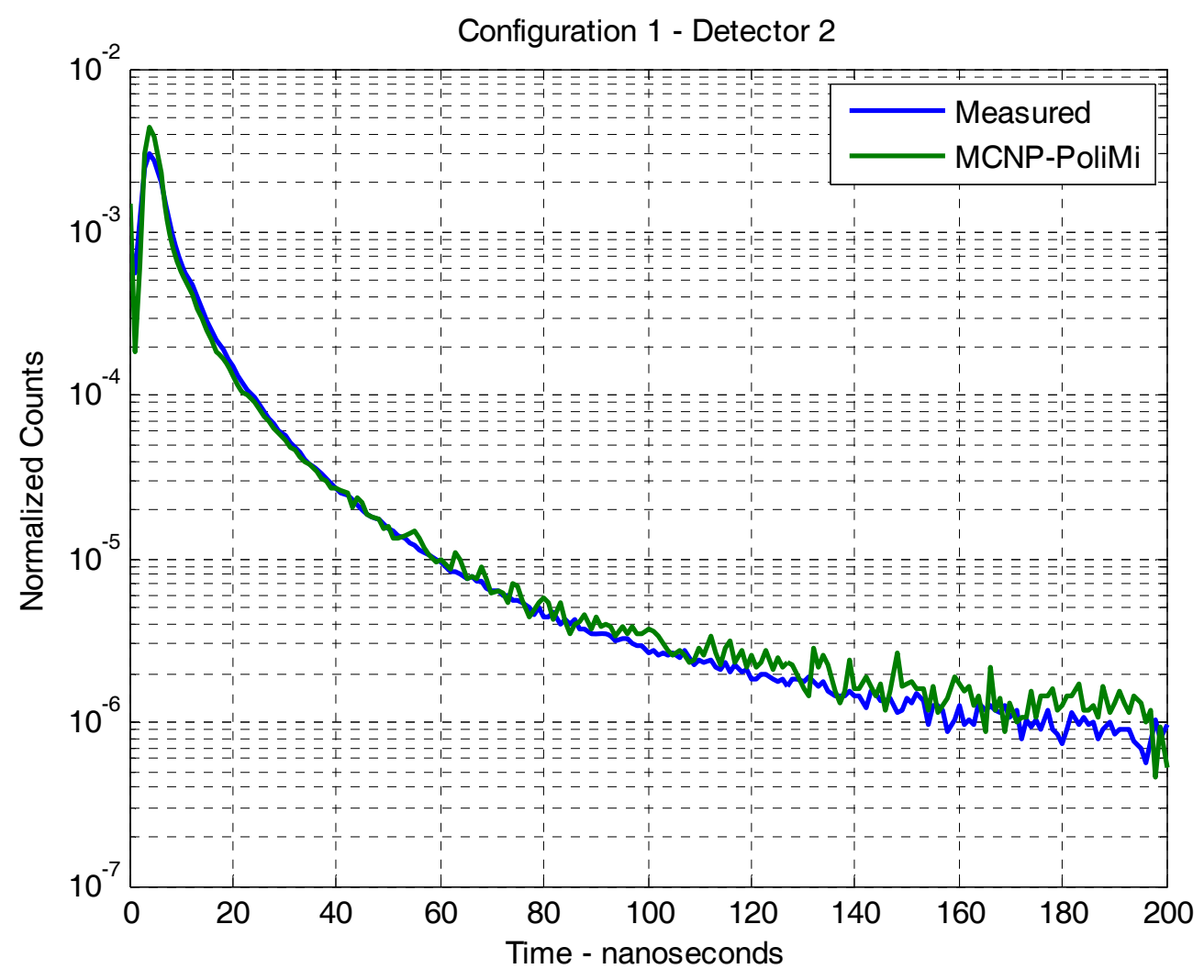

Fig. E.6. Time distribution of counts in detector 2 after $\mathbf{C f}$ fission for 200 ns for configuration 1. 


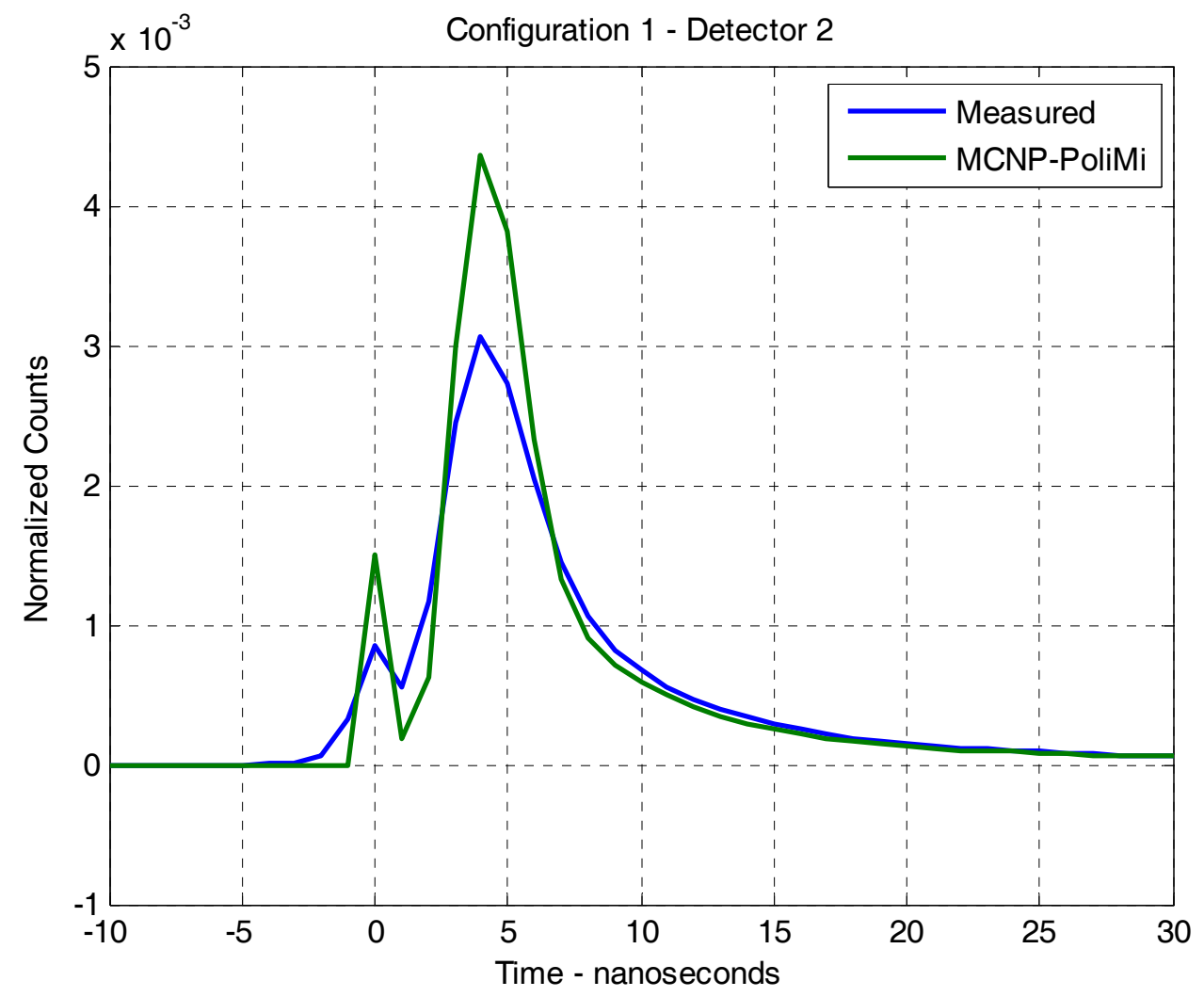

Fig. E.7. Time distribution of counts in detector 2 after $\mathbf{C f}$ fission for $\mathbf{3 0}$ ns for configuration 1. 


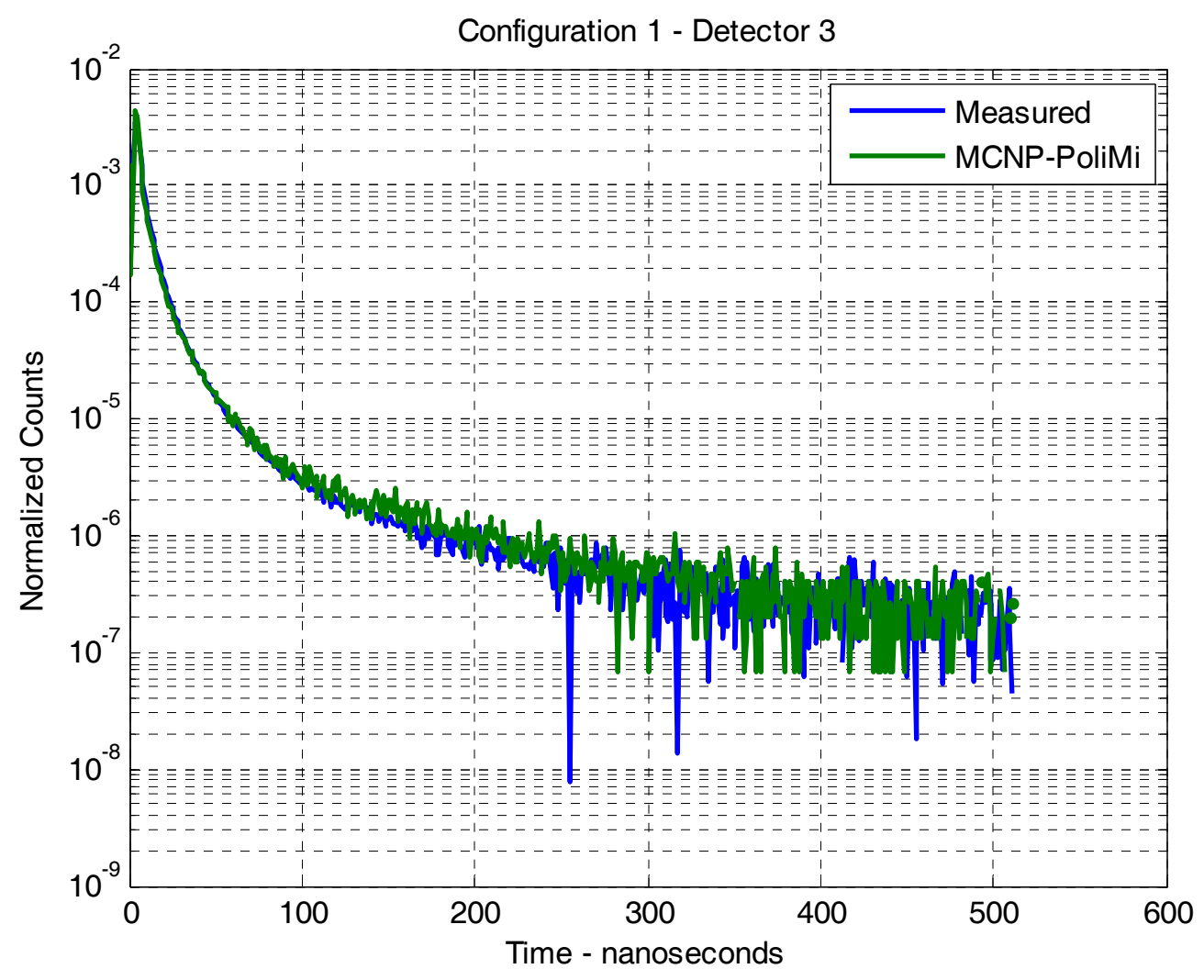

Fig. E.8. Time distribution of counts in detector 3 after $\mathbf{C f}$ fission for 512 ns for configuration 1. 


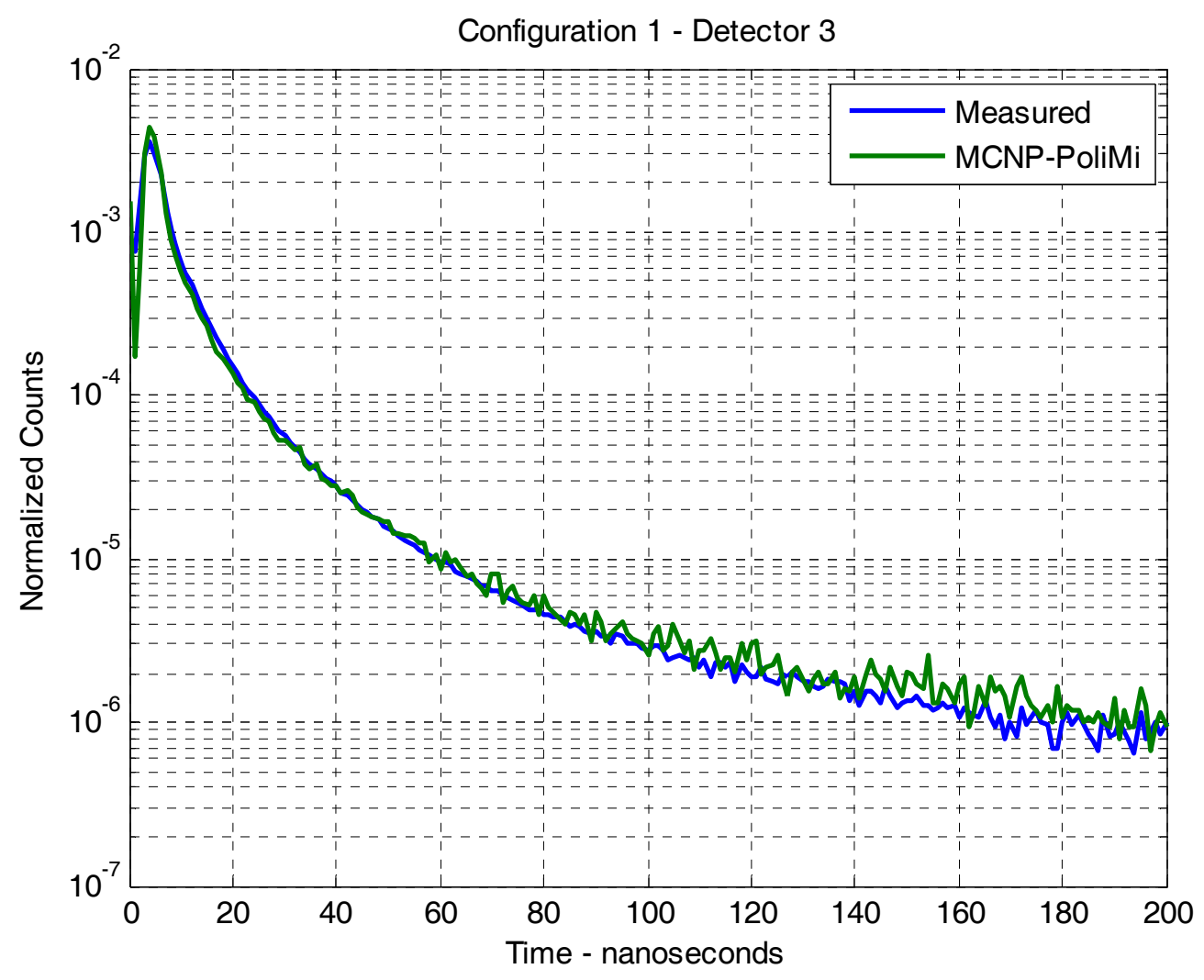

Fig. E.9. Time distribution of counts in detector 3 after $\mathbf{C f}$ fission for 200 ns for configuration 1. 


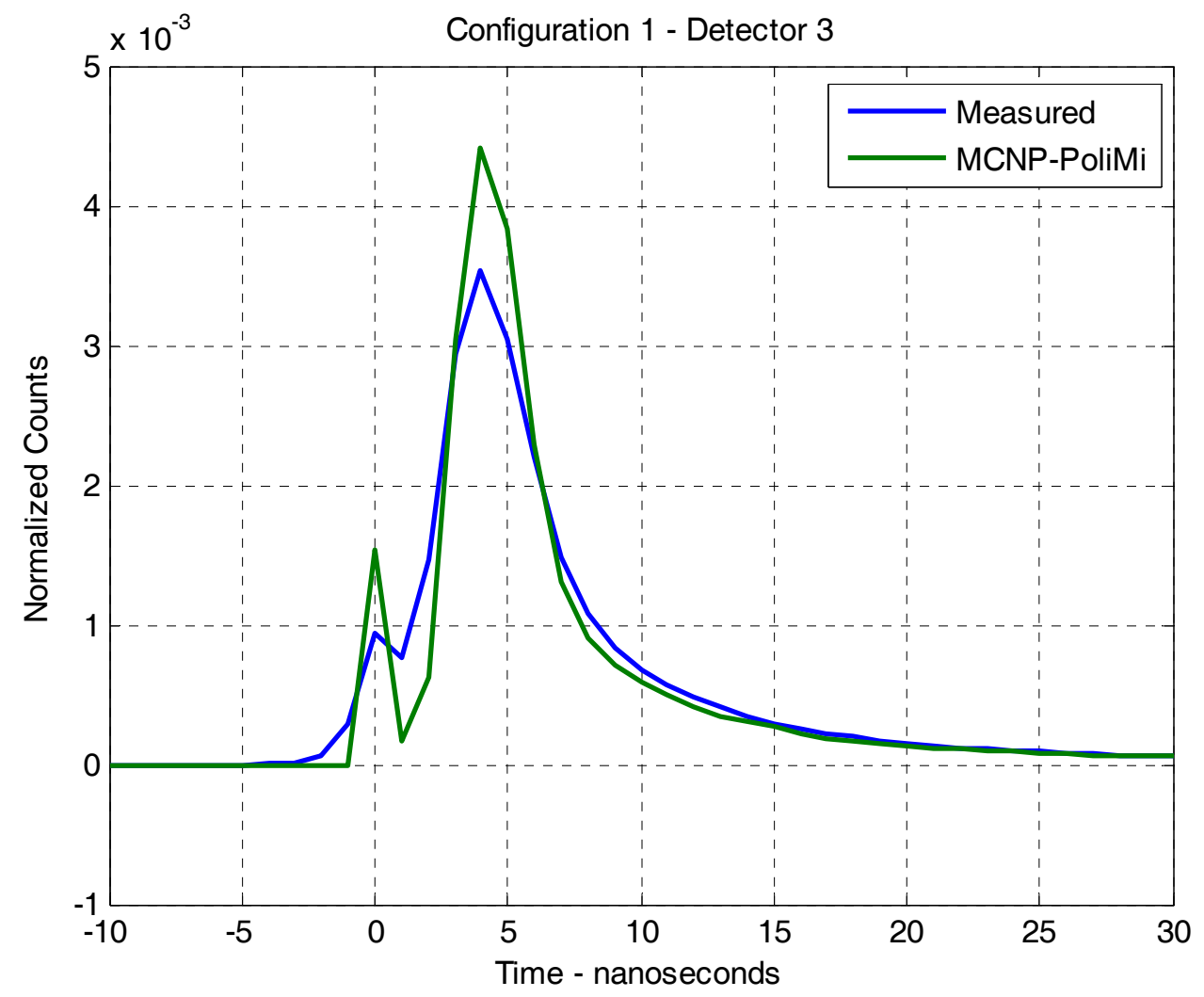

Fig. E.10. Time distribution of counts in detector 3 after $\mathbf{C f}$ fission for $\mathbf{3 0}$ ns for configuration 1. 


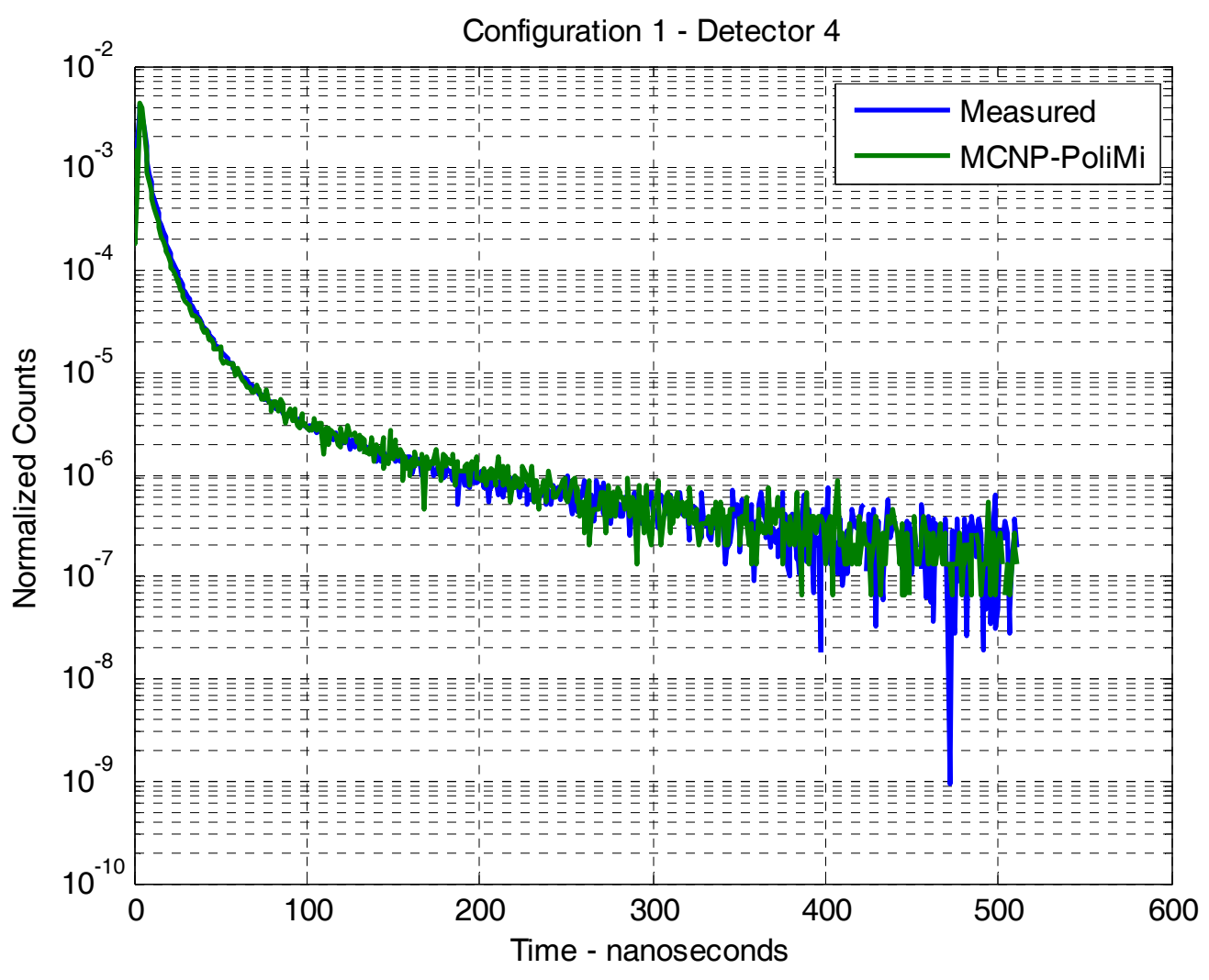

Fig. E.11. Time distribution of counts in detector 4 after $\mathbf{C f}$ fission for 512 ns for configuration 1. 


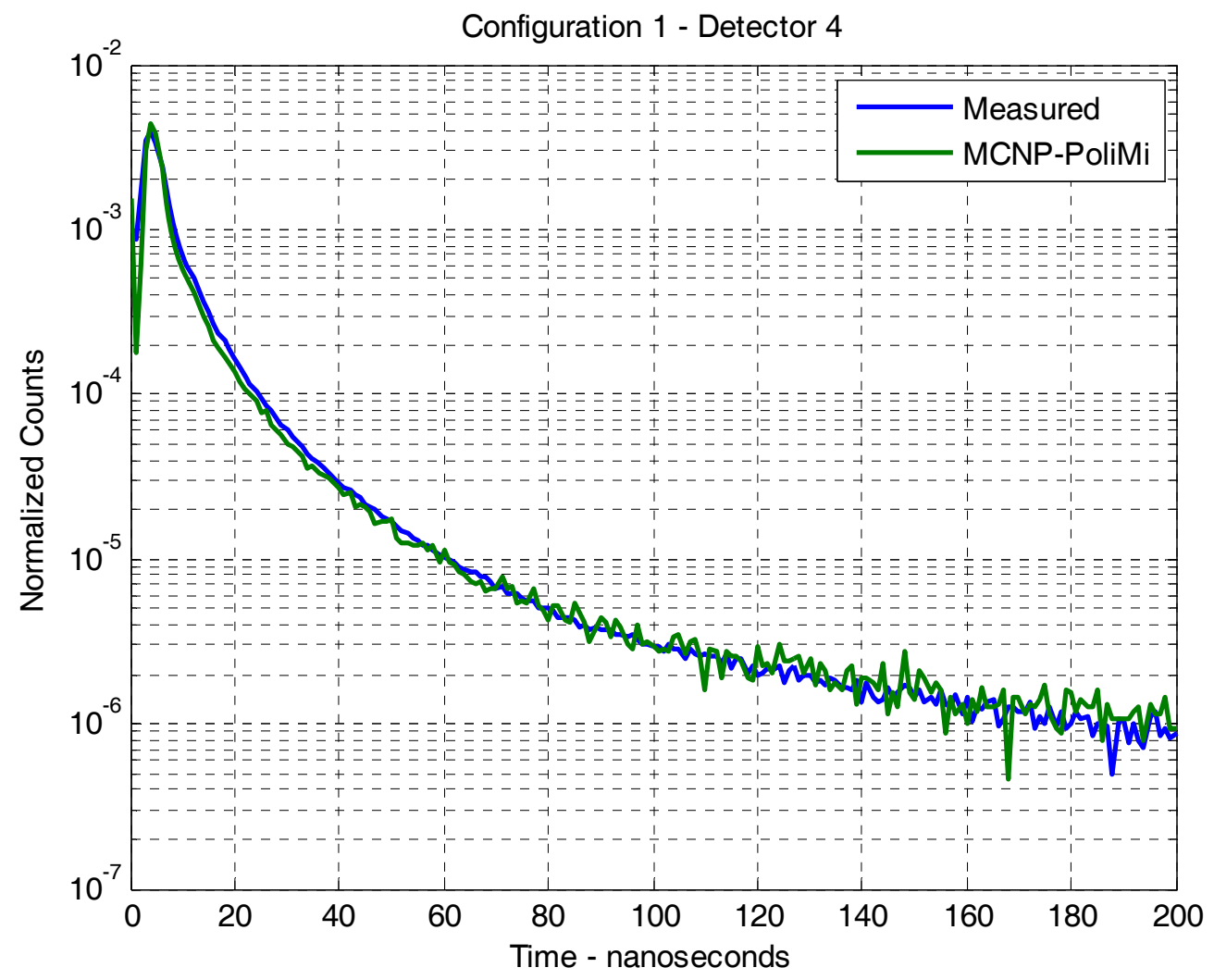

Fig. E.12. Time distribution of counts in detector1 after Cf fission for $200 \mathrm{~ns}$ for configuration 1. 


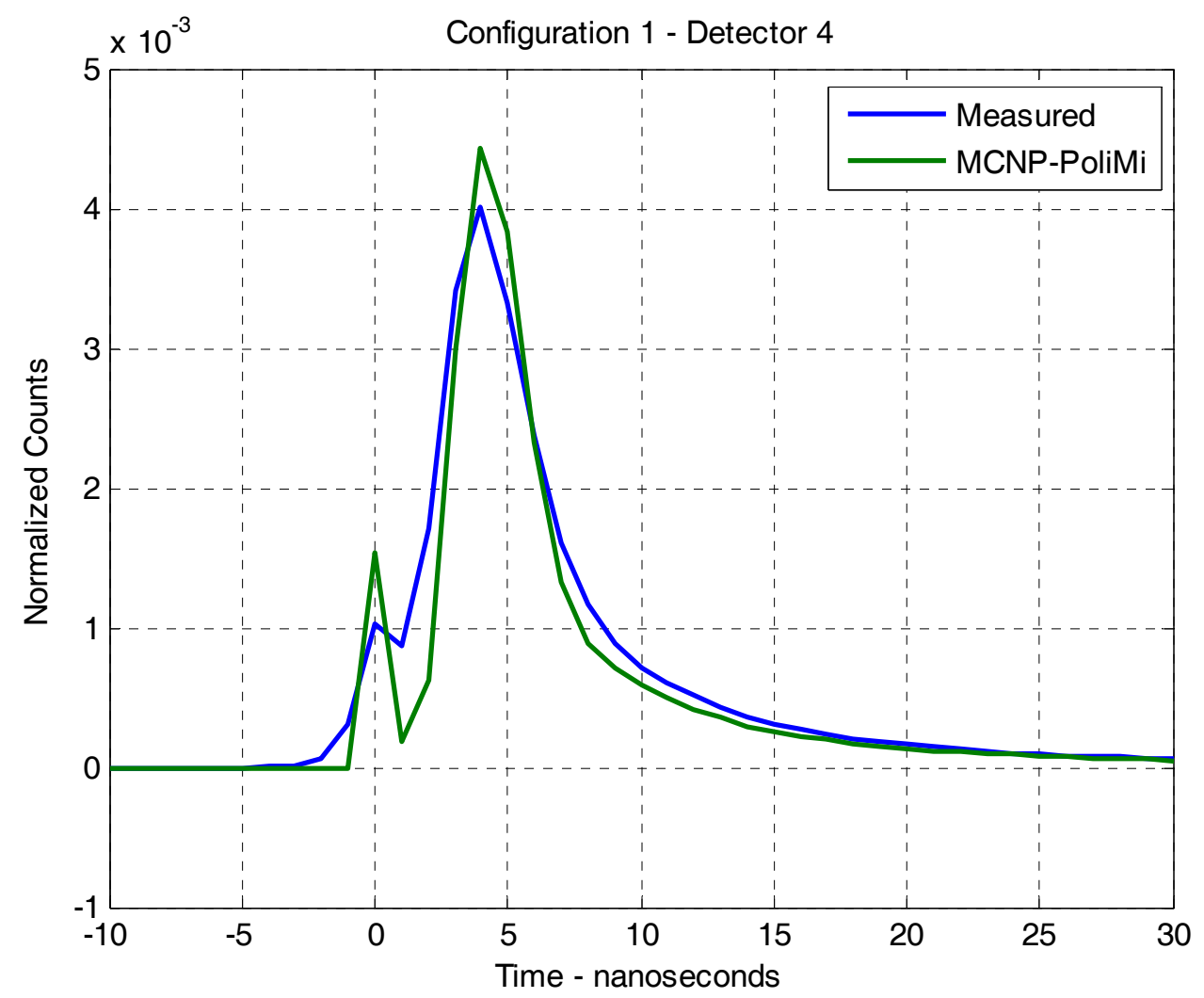

Fig. E.13. Time distribution of counts in detector 1 after $\mathbf{C f}$ fission for $\mathbf{3 0}$ ns for configuration 1. 


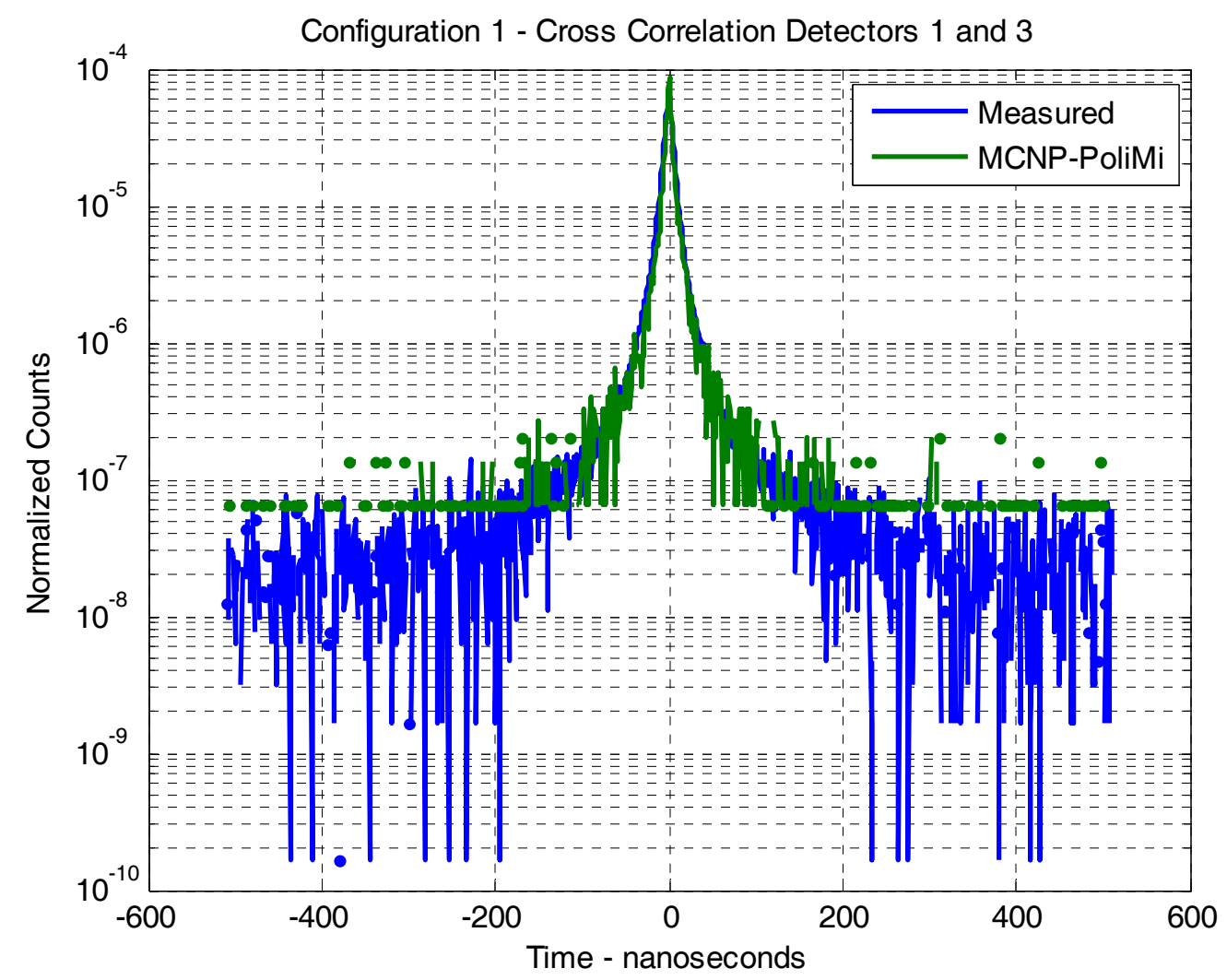

Fig. E.14. Time distribution of counts in detectors 3 after a count in detector 1 for 512 ns for configuration 1. 


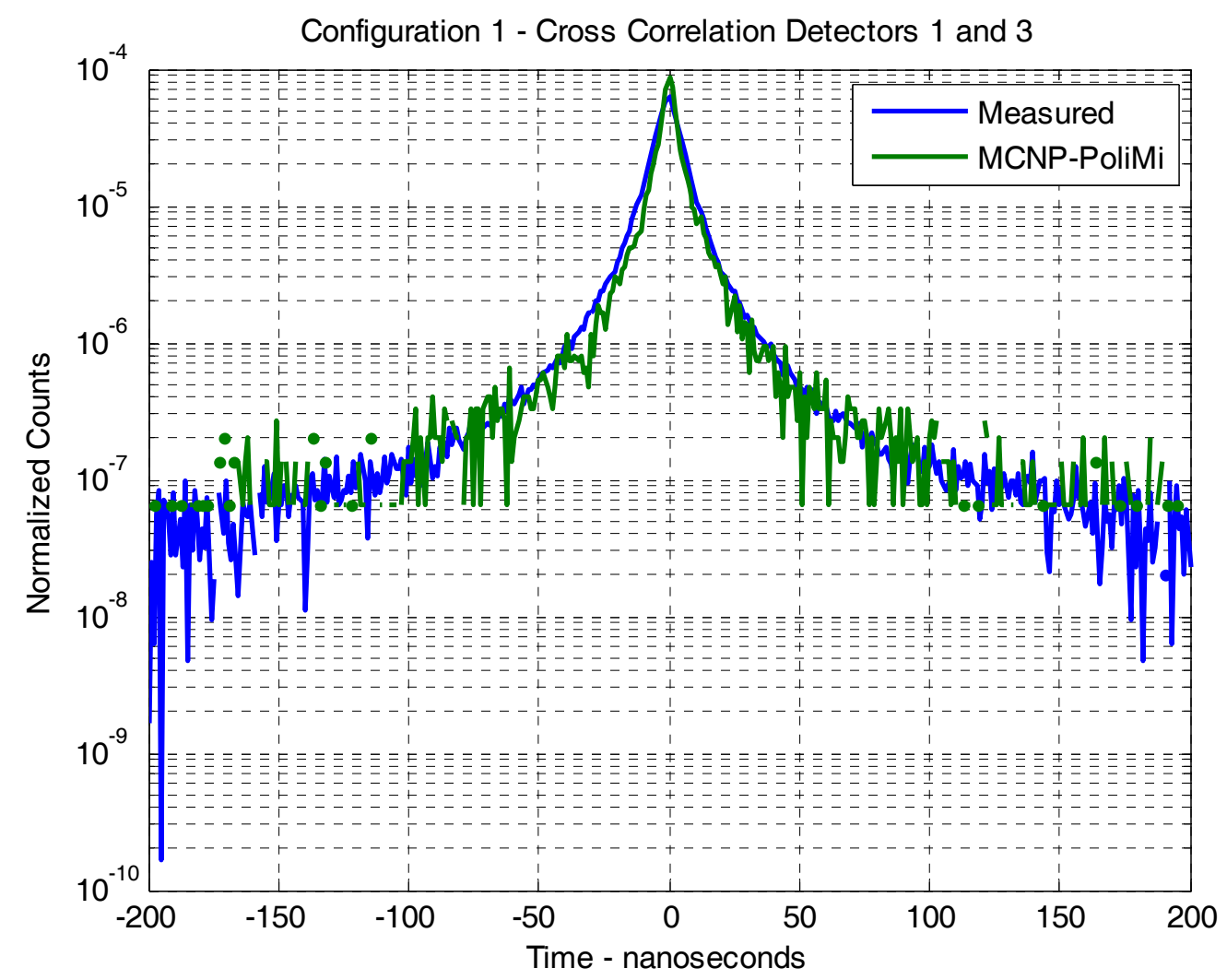

Fig. E.15. Time distribution of counts in detectors 3 after a count in detector 1 for $200 \mathrm{~ns}$ for configuration 1. 


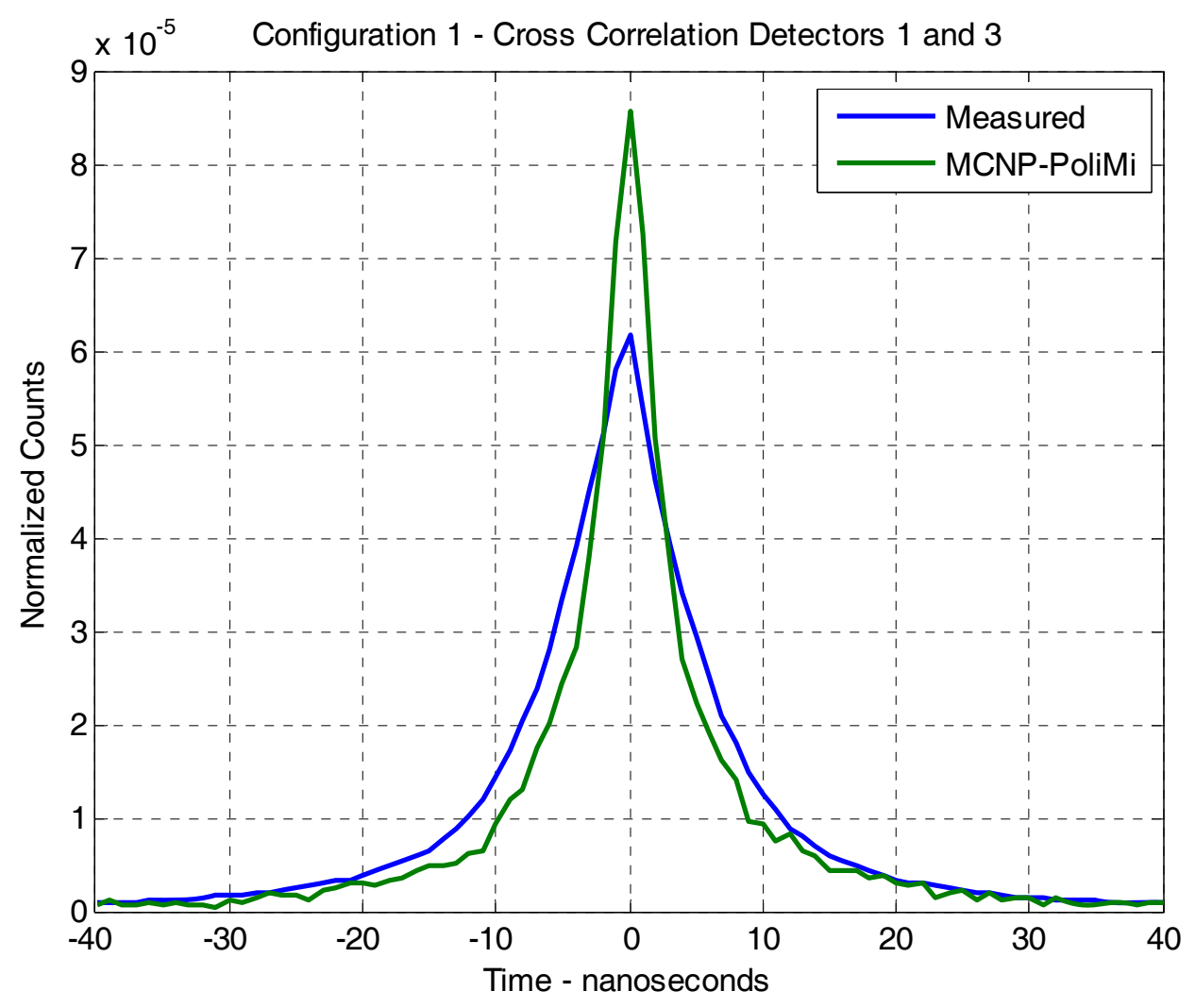

Fig. E.16. Time distribution of counts in detectors 3 after a count in detector 1 for 30 ns for configuration 1. 
Configuration 3:

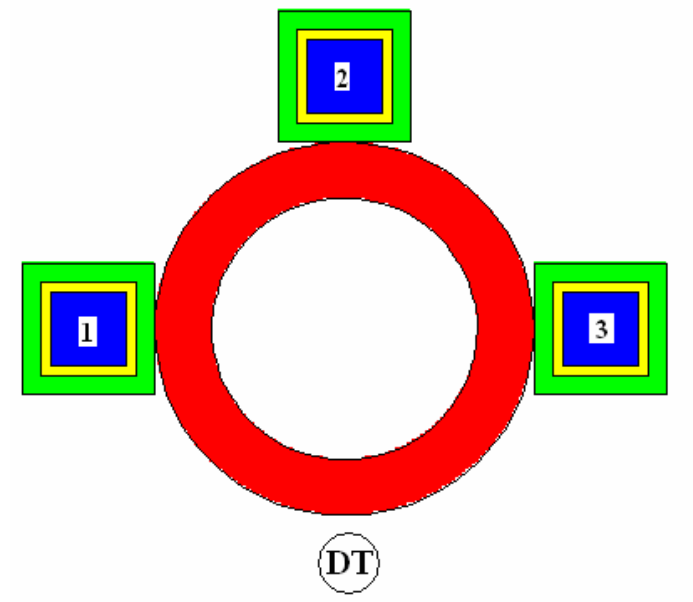

CONFIGURATION 3

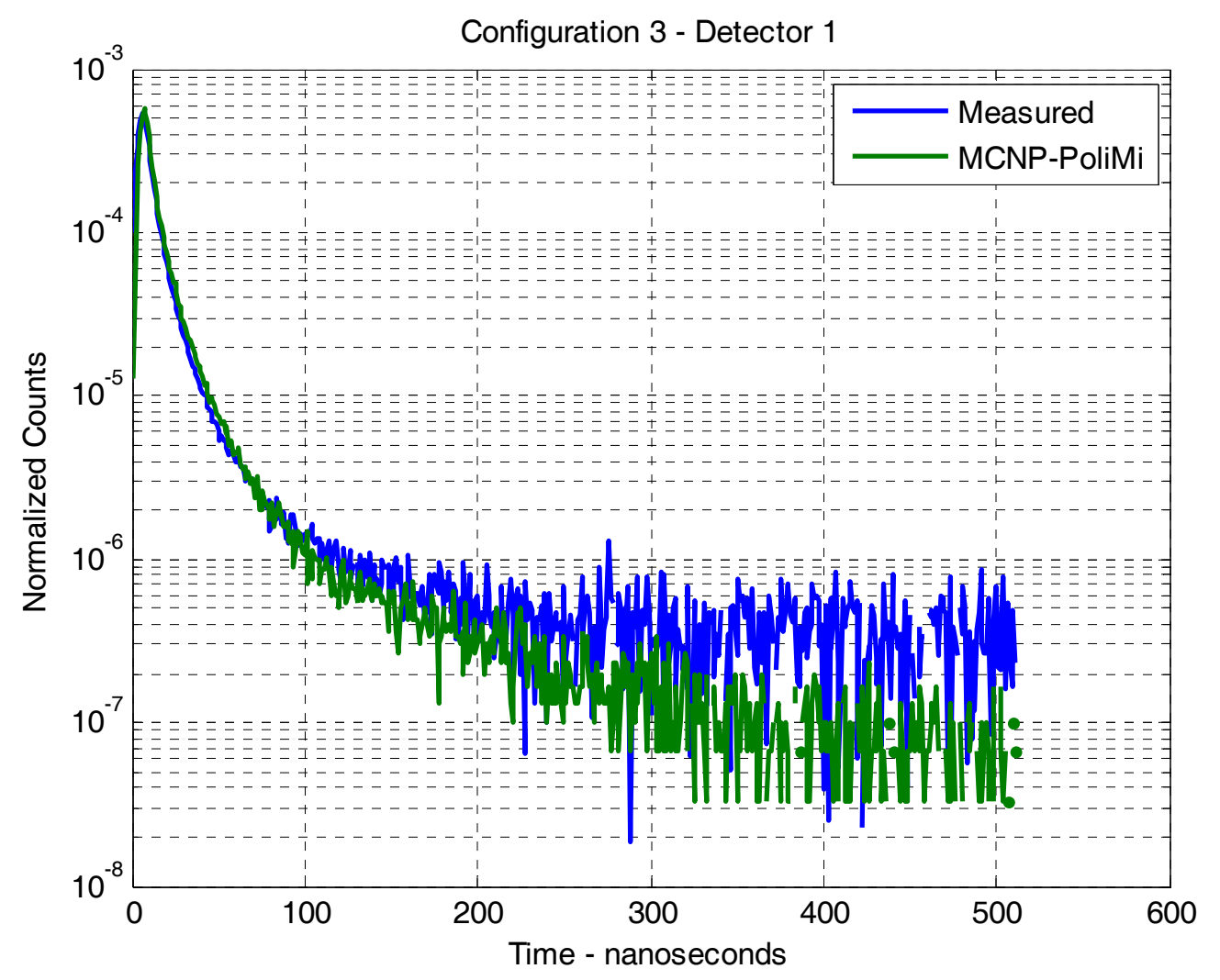

Fig. E.17. Time distribution of counts in detector 1 after $\mathbf{C f}$ fission for 512 ns for configuration 3. 


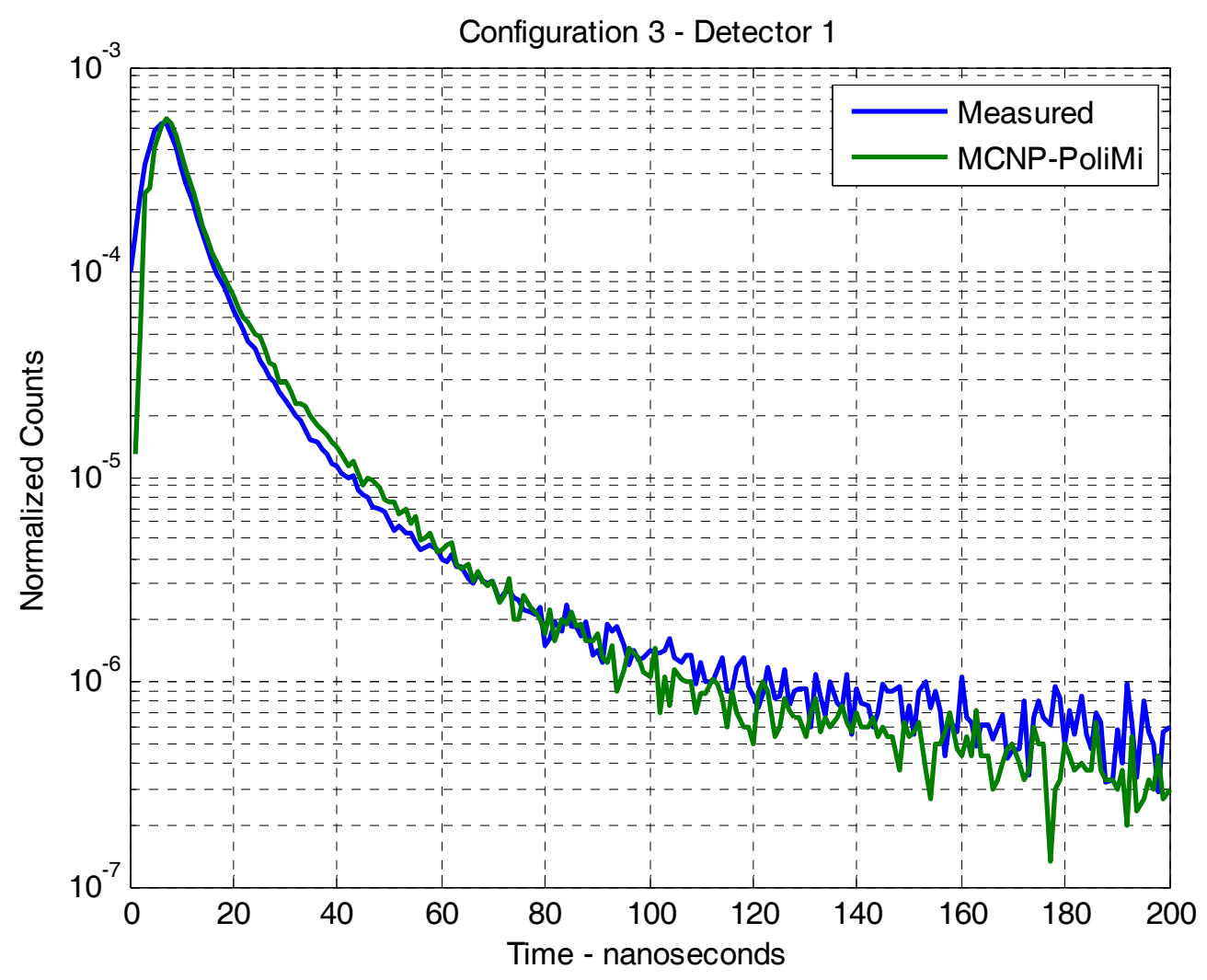

Fig. E.18. Time distribution of counts in detector1 after $\mathbf{C f}$ fission for $200 \mathrm{~ns}$ for configuration 3. 


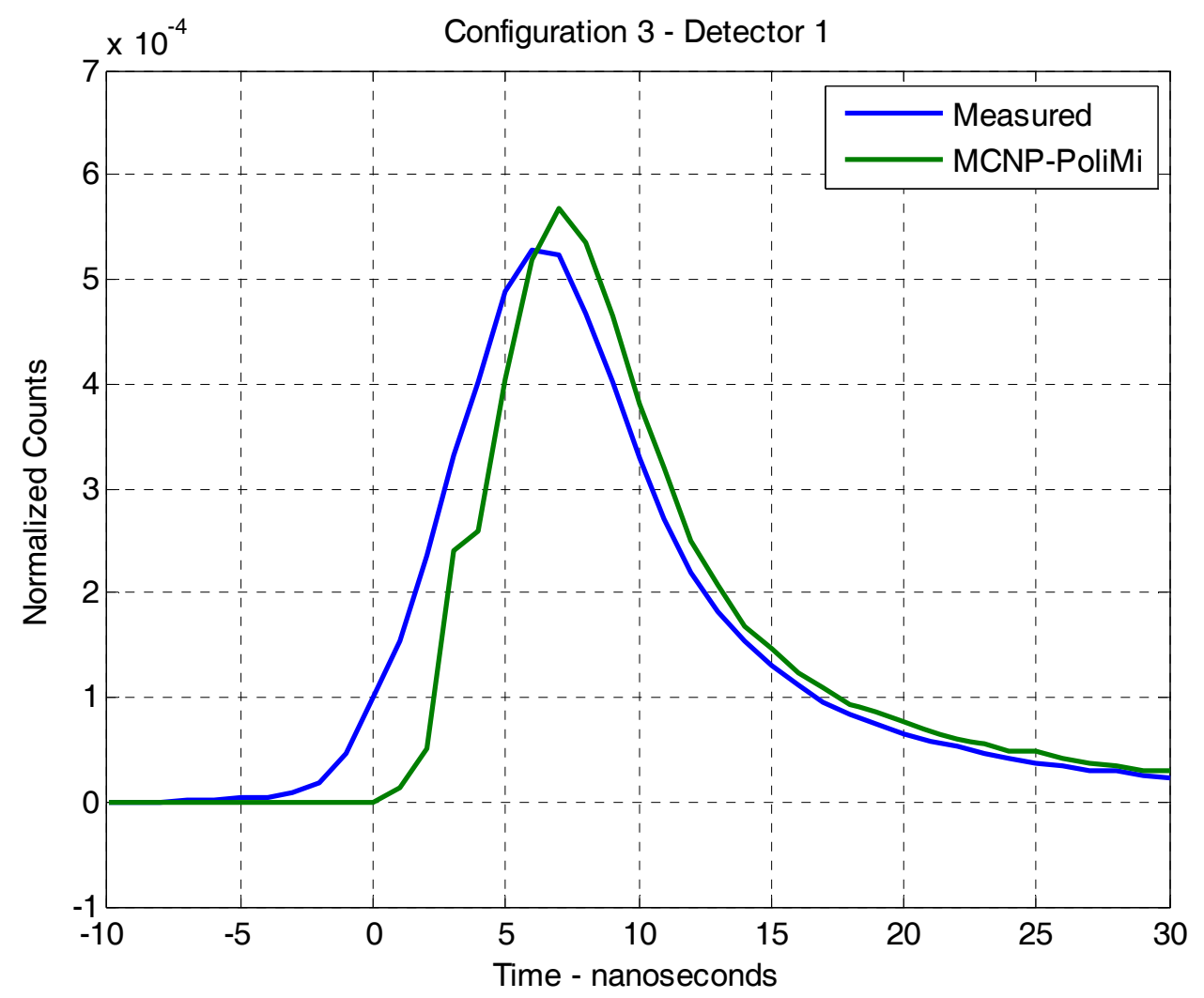

Fig. E.19. Time distribution of counts in detector 3 after $\mathbf{C f}$ fission for $\mathbf{3 0}$ ns for configuration 3. 


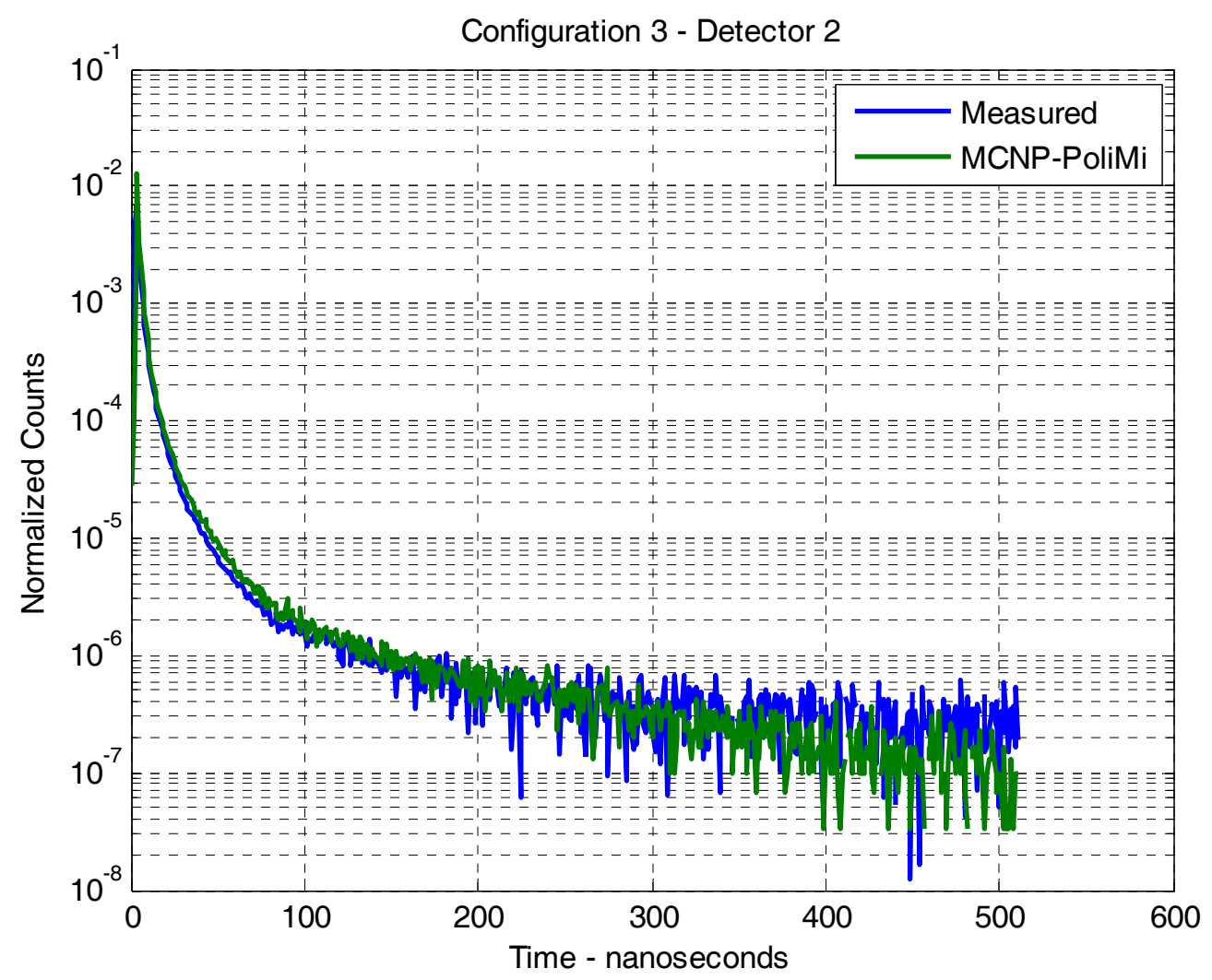

Fig. E.20. Time distribution of counts in detector 2 after Cf fission for 512 ns for configuration 3. 


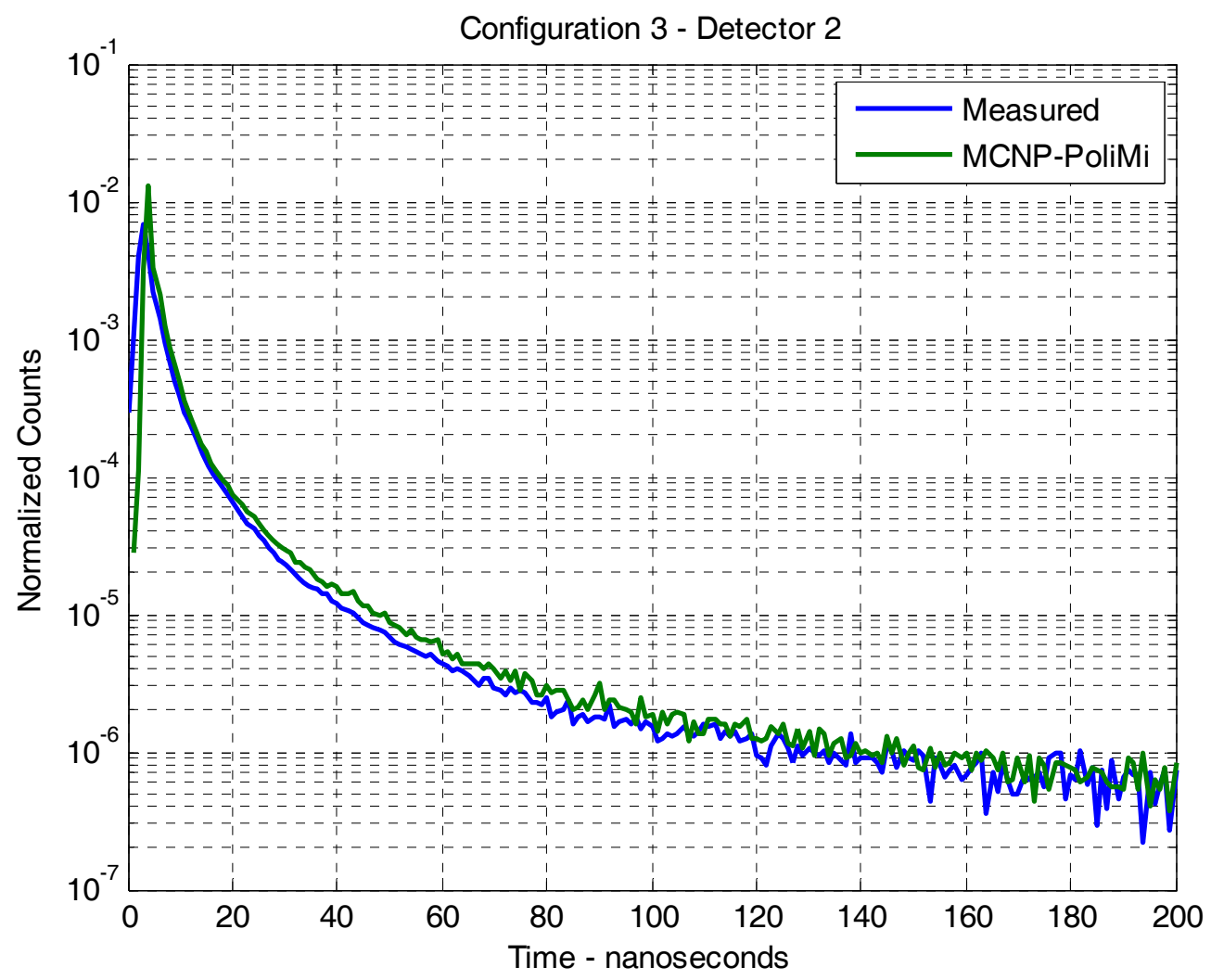

Fig. E.21. Time distribution of counts in detector 2 after $\mathbf{C f}$ fission for $200 \mathrm{~ns}$ for configuration 3. 


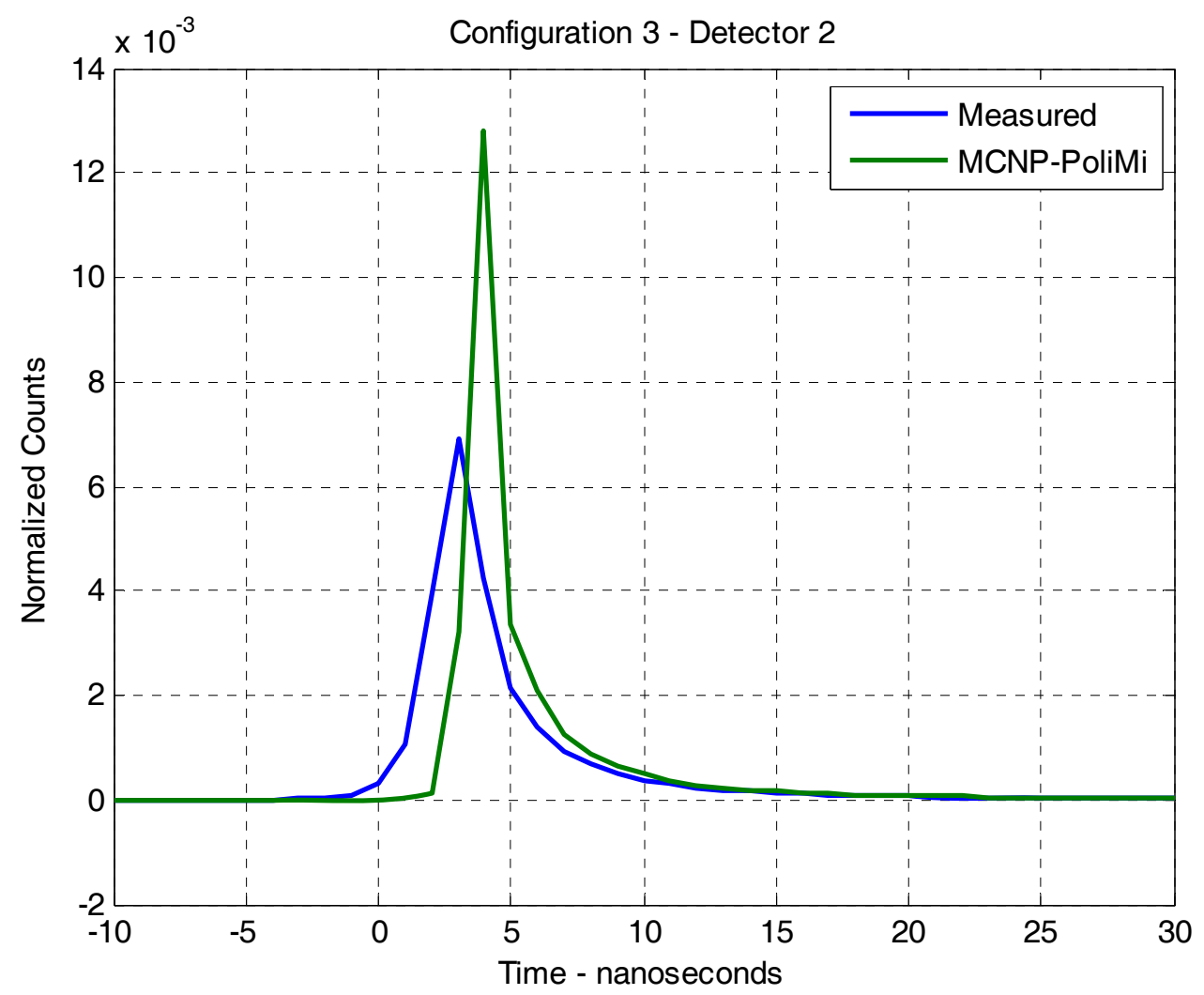

Fig. E.22. Time distribution of counts in detector $\mathbf{2}$ after $\mathbf{C f}$ fission for $\mathbf{3 0}$ ns for configuration 3. 


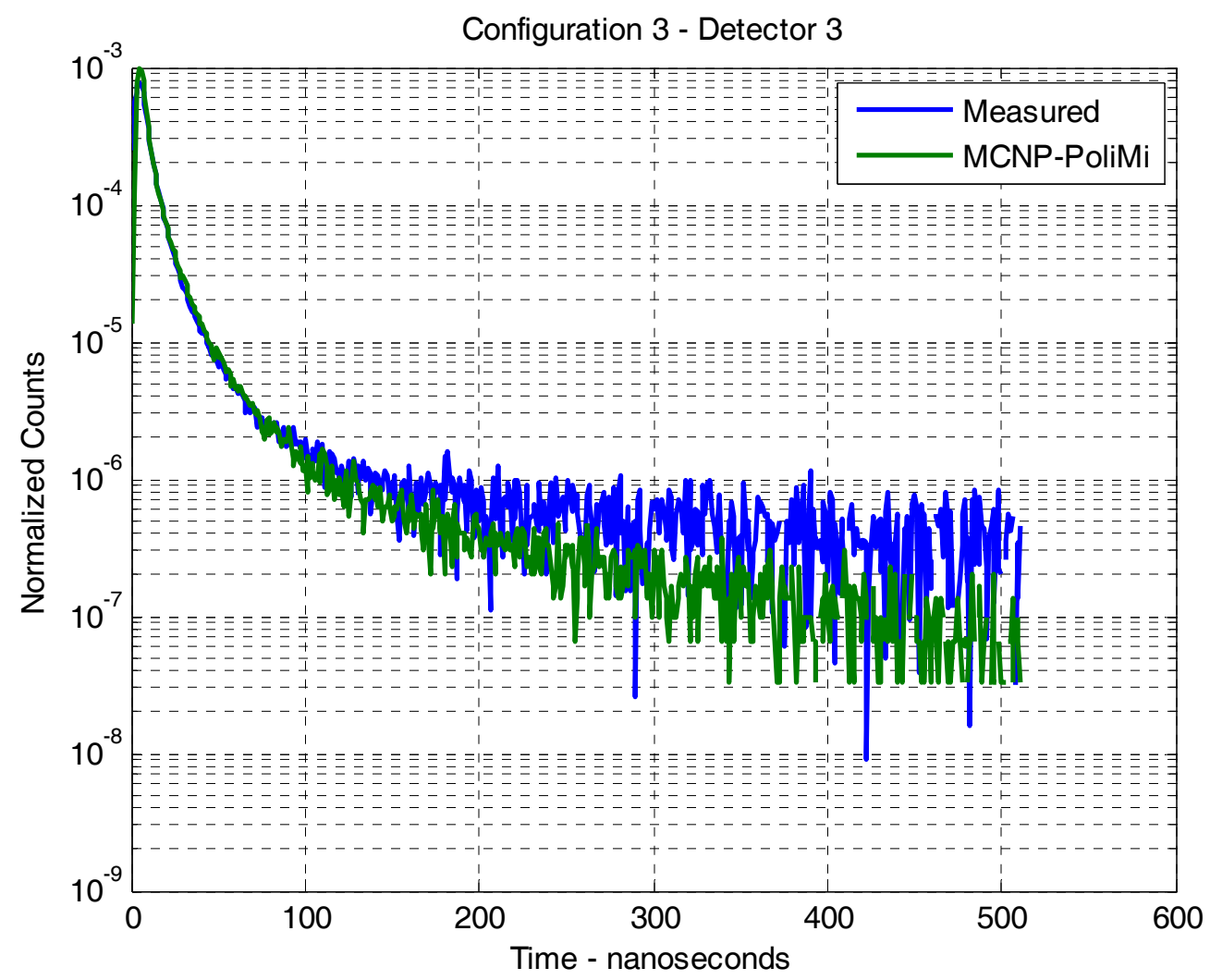

Fig. E.23. Time distribution of counts in detector 3 after $\mathbf{C f}$ fission for 512 ns for configuration 3. 


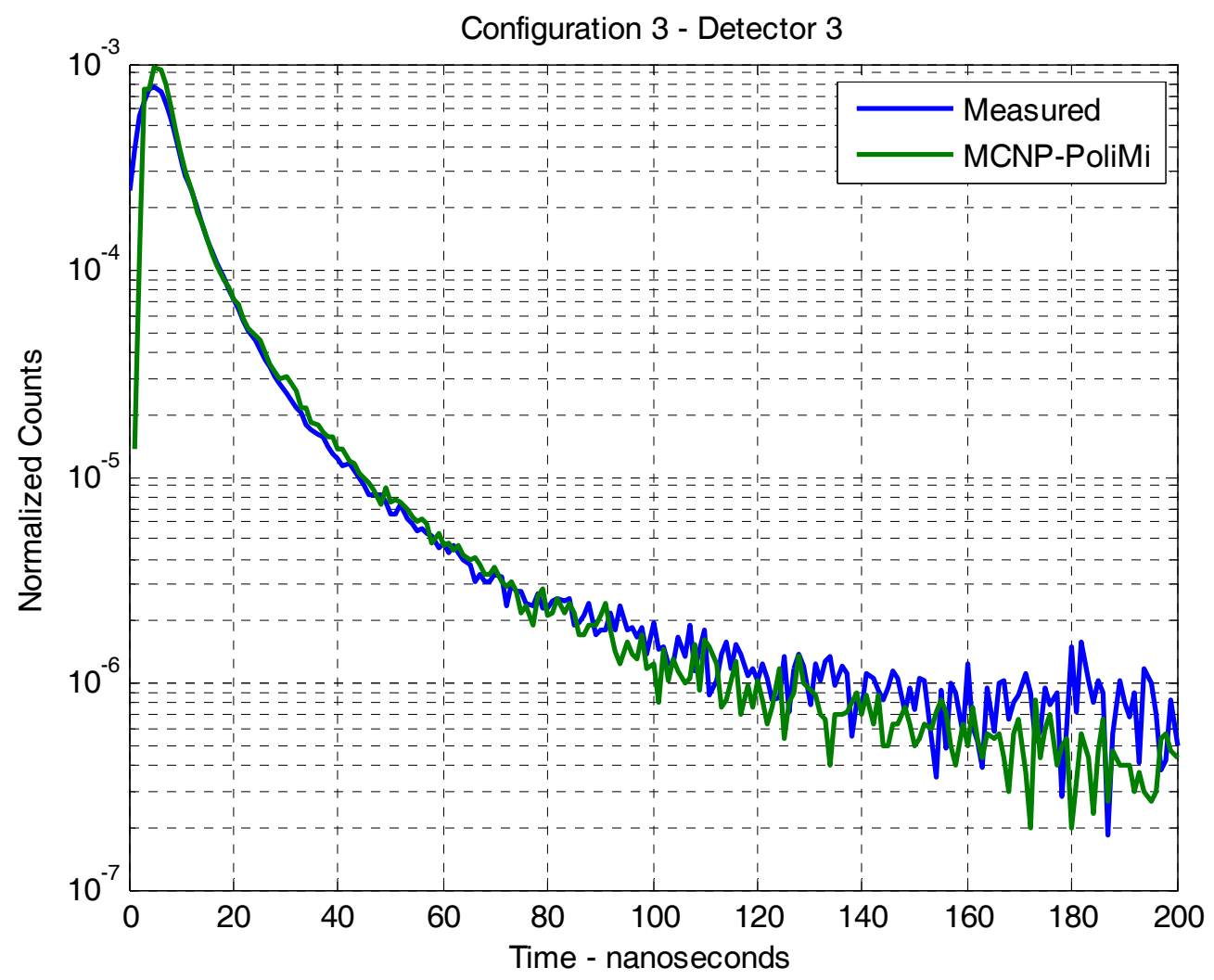

Fig. E.24. Time distribution of counts in detector 3 after $\mathbf{C f}$ fission for $200 \mathrm{~ns}$ for configuration 3. 


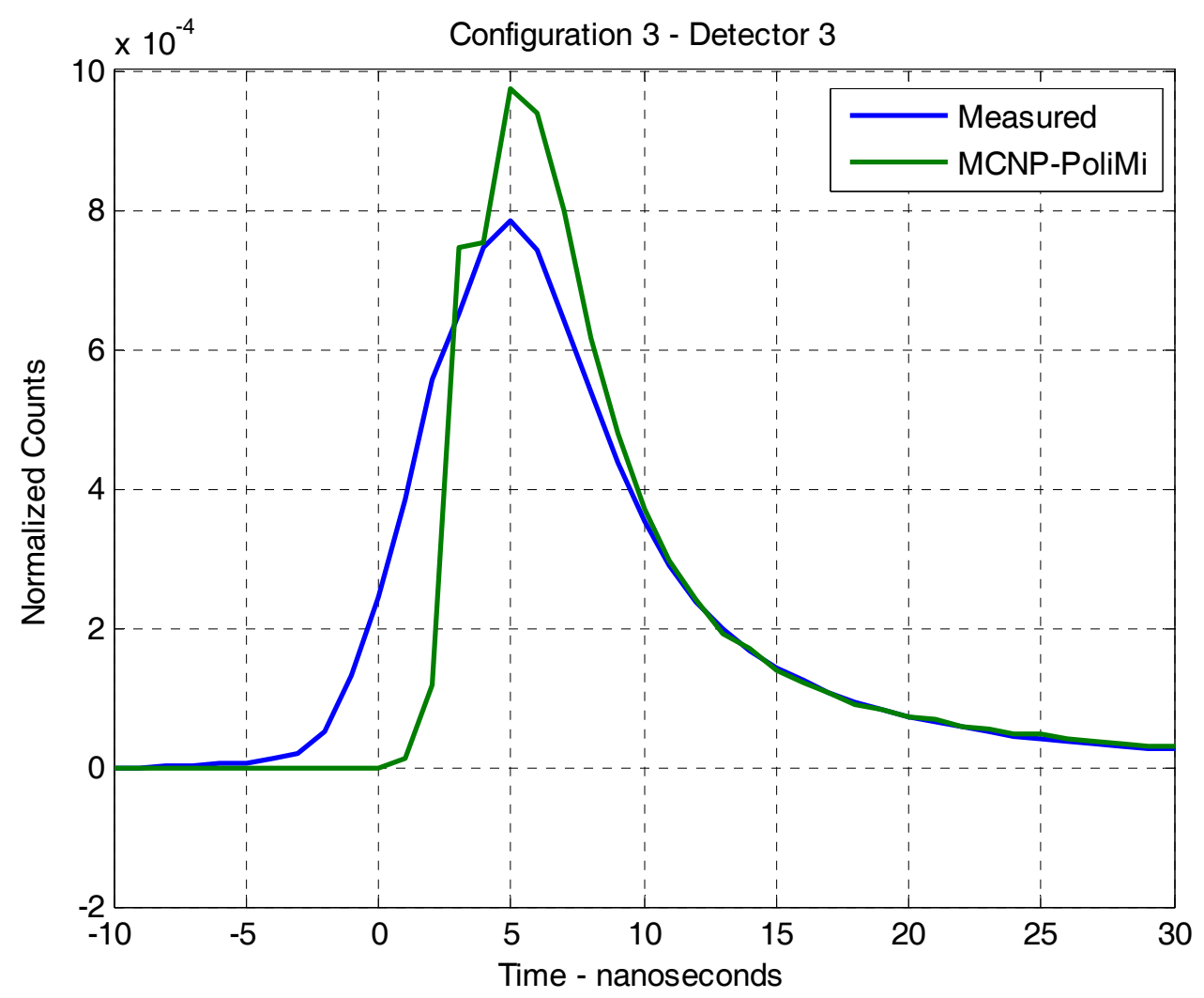

Fig. E.25. Time distribution of counts in detector 3 after $\mathbf{C f}$ fission for $\mathbf{3 0}$ ns for configuration 3. 
Configuration 4:

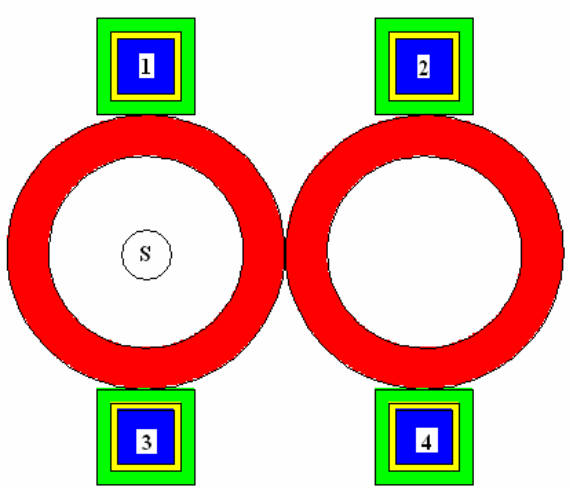

CONFIGURATION 4

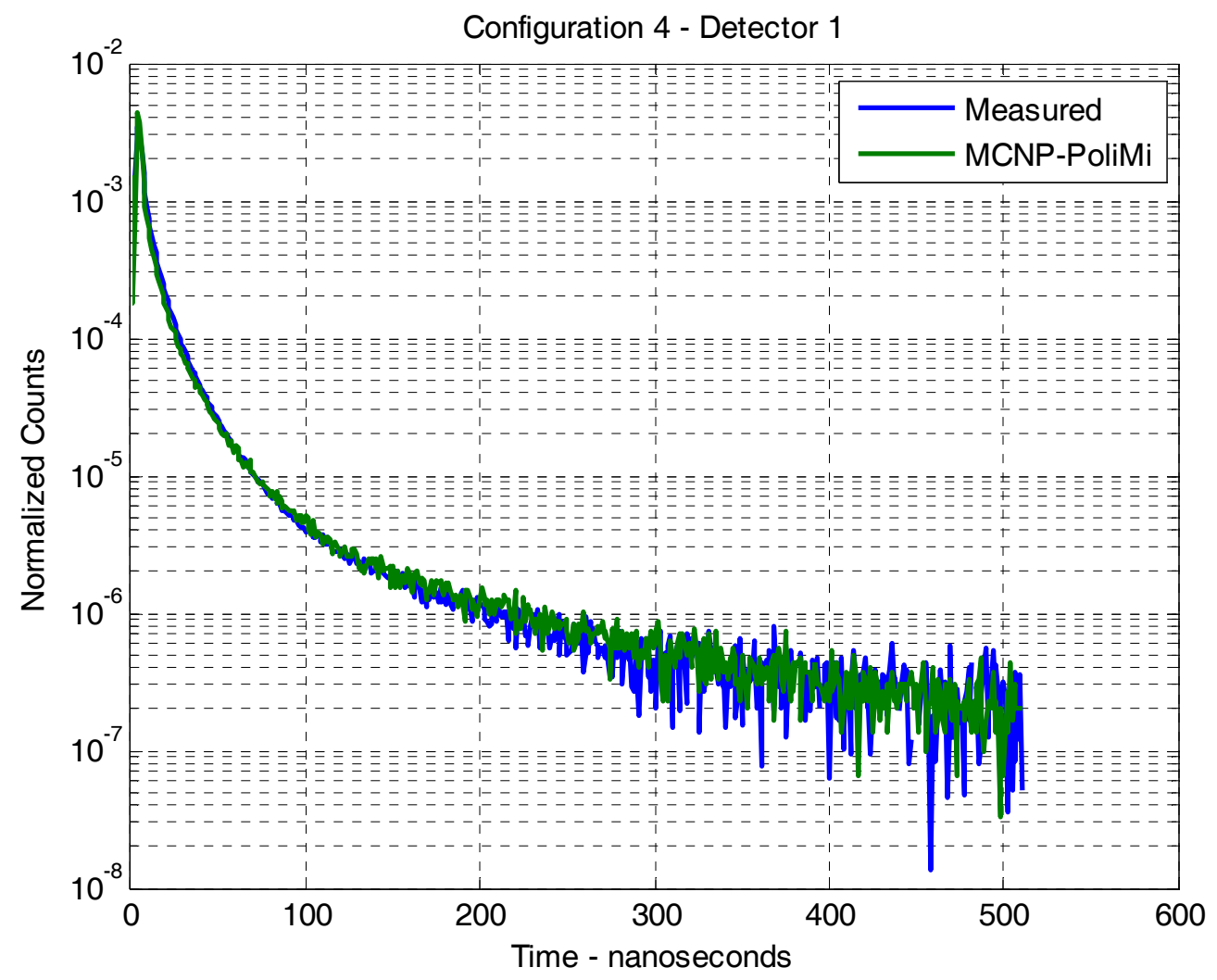

Fig. E.26. Time distribution of counts in detector 1 after Cf fission for 512 ns for configuration 4. 


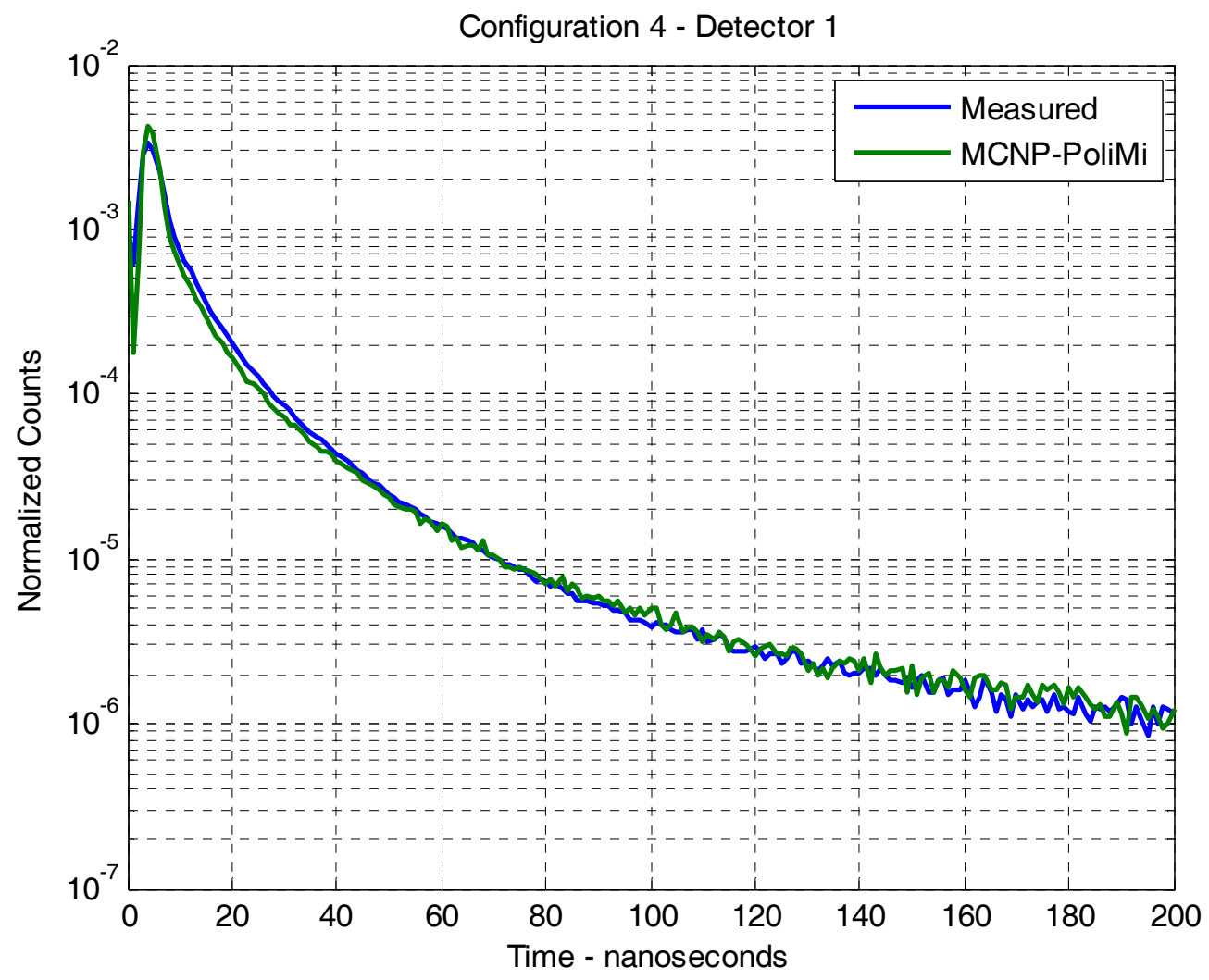

Fig. E.27. Time distribution of counts in detector 1 after $\mathbf{C f}$ fission for $200 \mathrm{~ns}$ for configuration 4. 


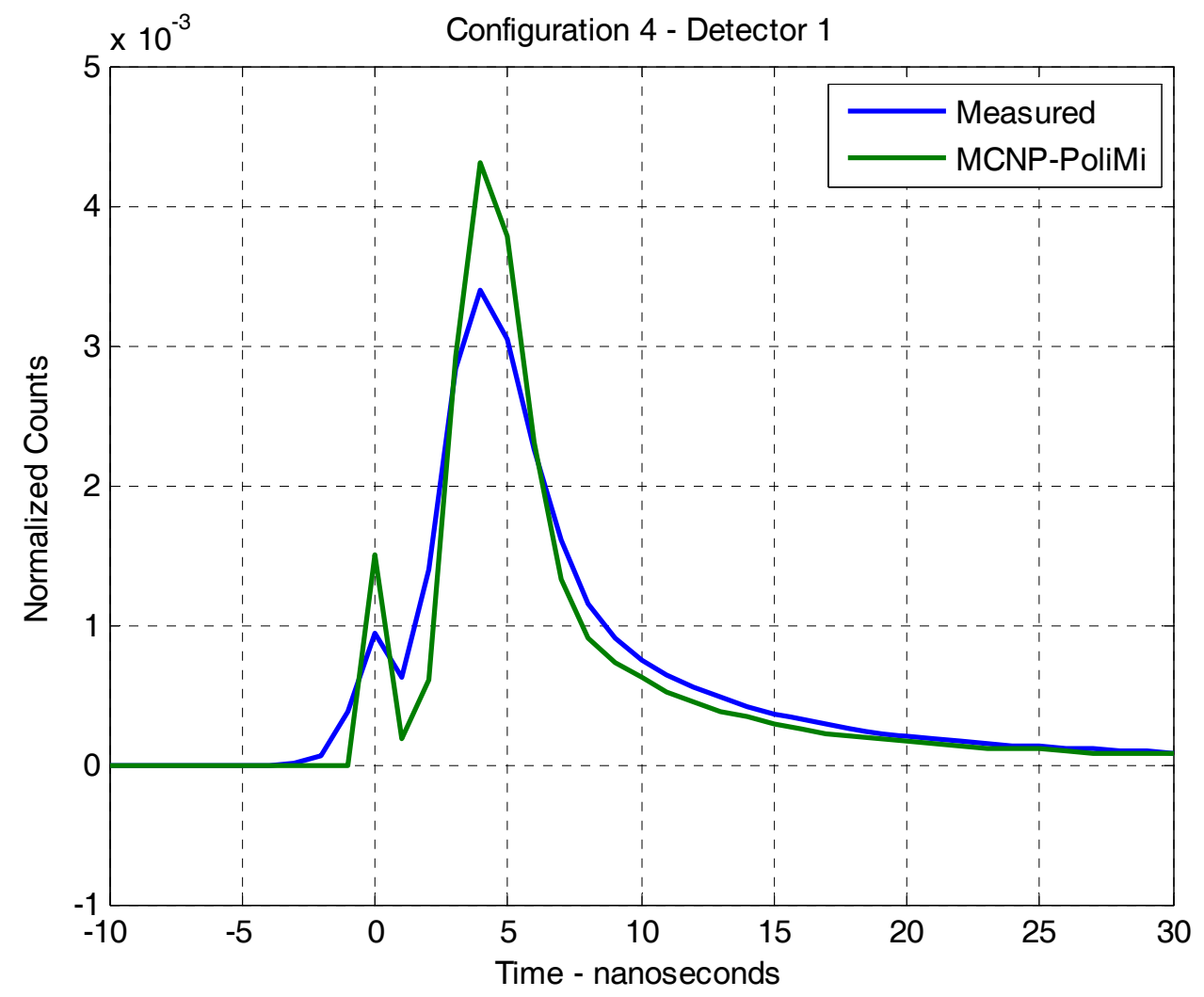

Fig. E.28. Time distribution of counts in detector 1 after $\mathbf{C f}$ fission for $\mathbf{3 0}$ ns for configuration 4. 


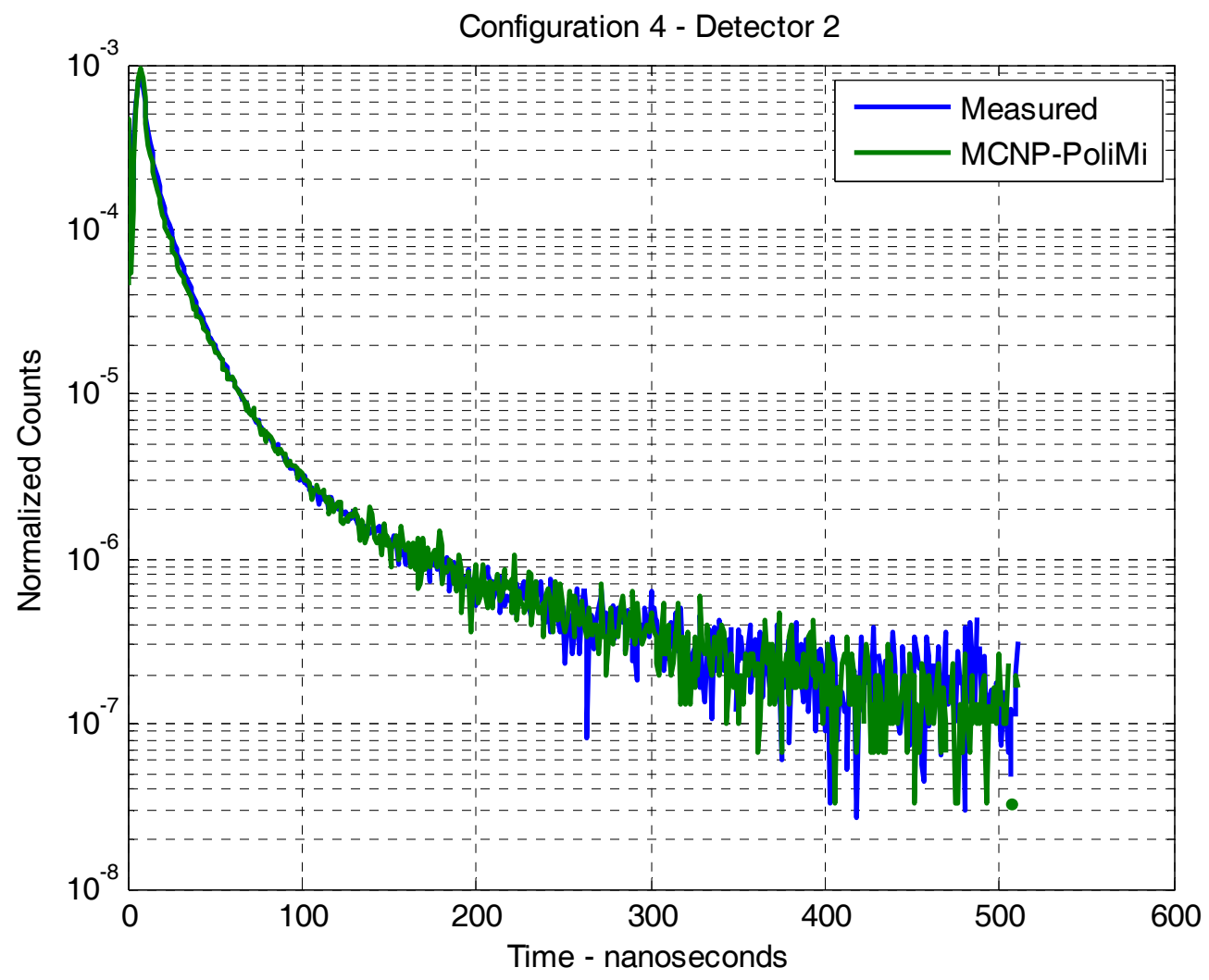

Fig. E.29. Time distribution of counts in detector 2 after Cf fission for 512 ns for configuration 4. 


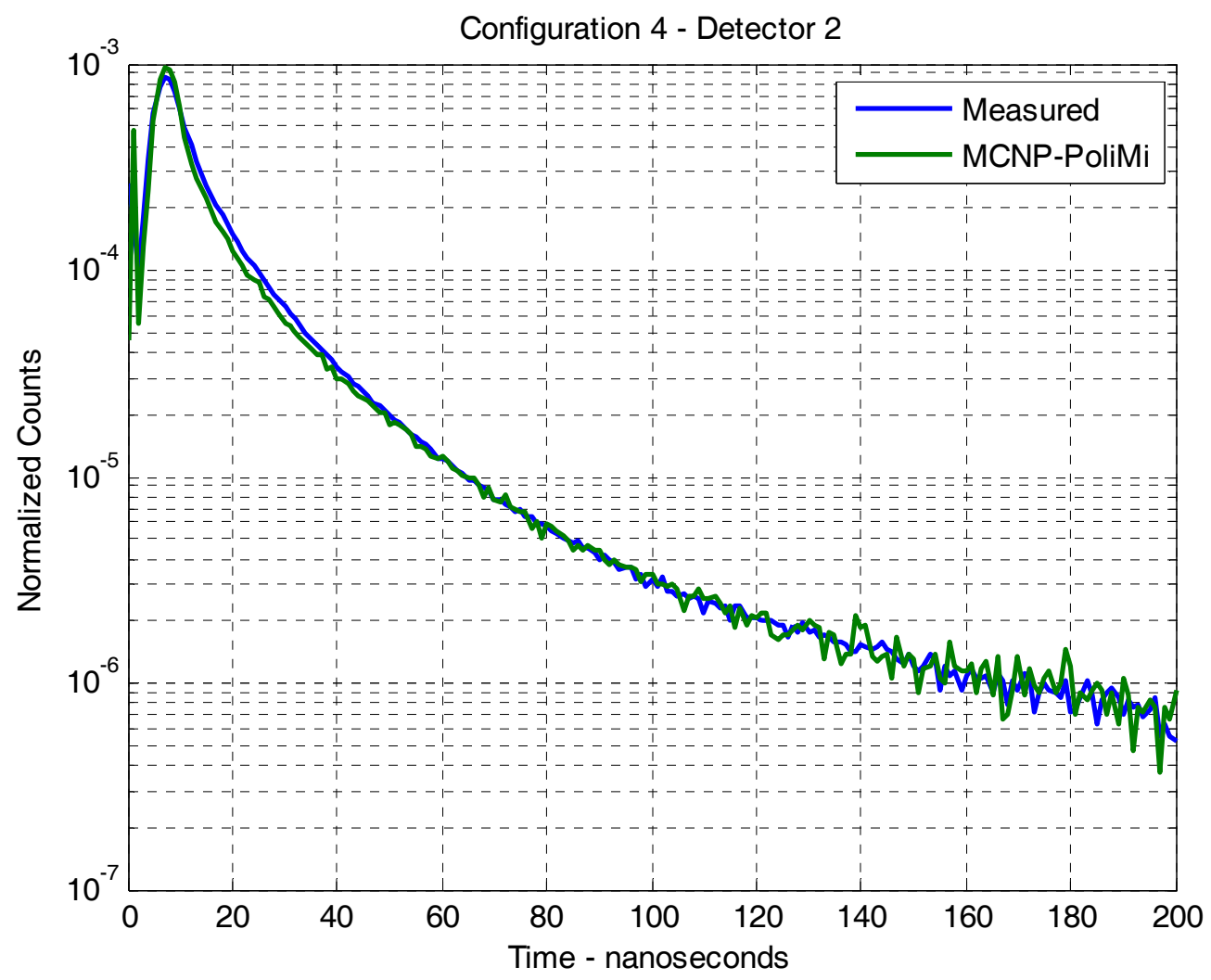

Fig. E.30. Time distribution of counts in detector 2 after Cf fission for 200 ns for configuration 4. 


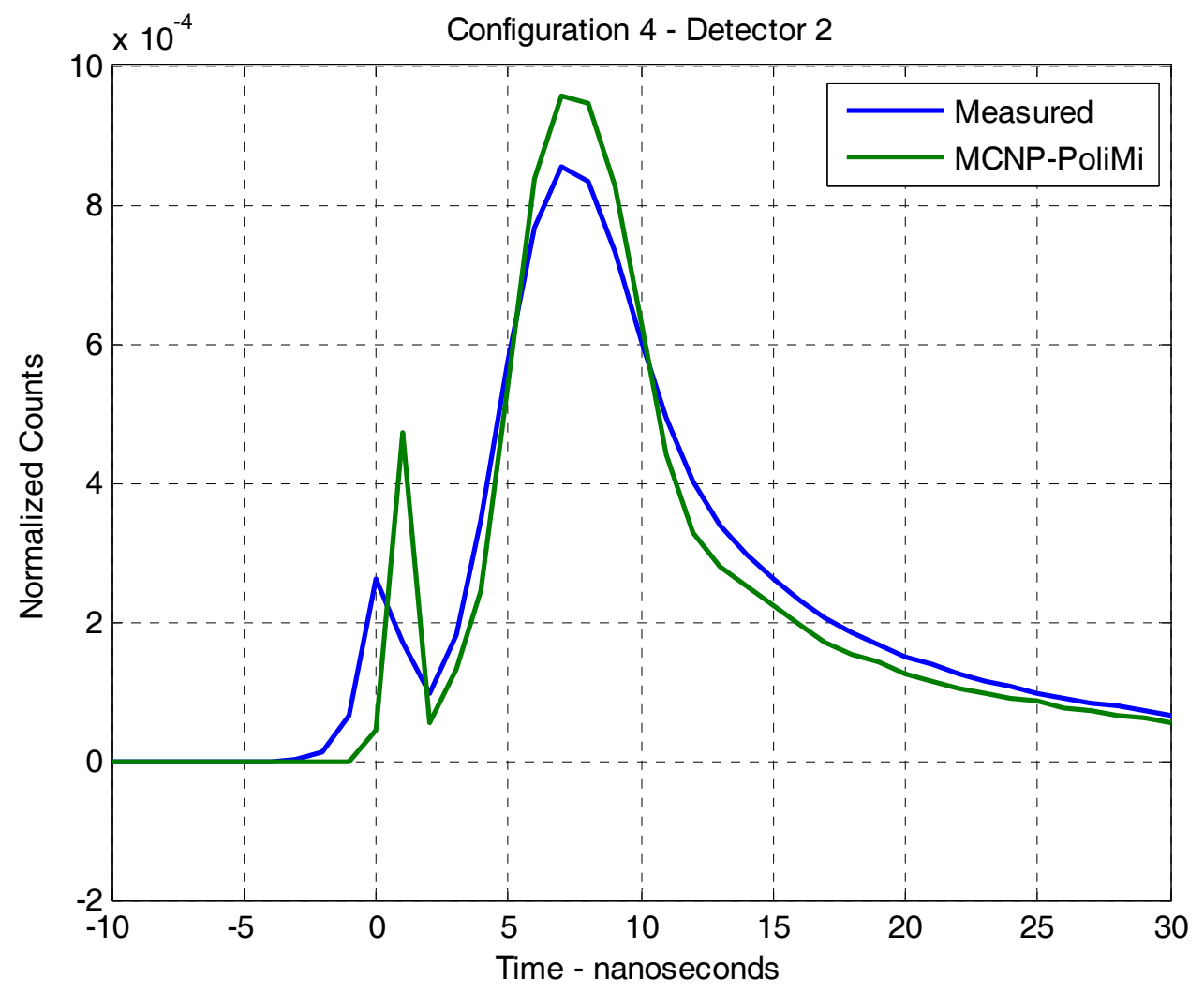

Fig. E.31. Time distribution of counts in detector 2 after $\mathbf{C f}$ fission for 30 ns for configuration 4. 


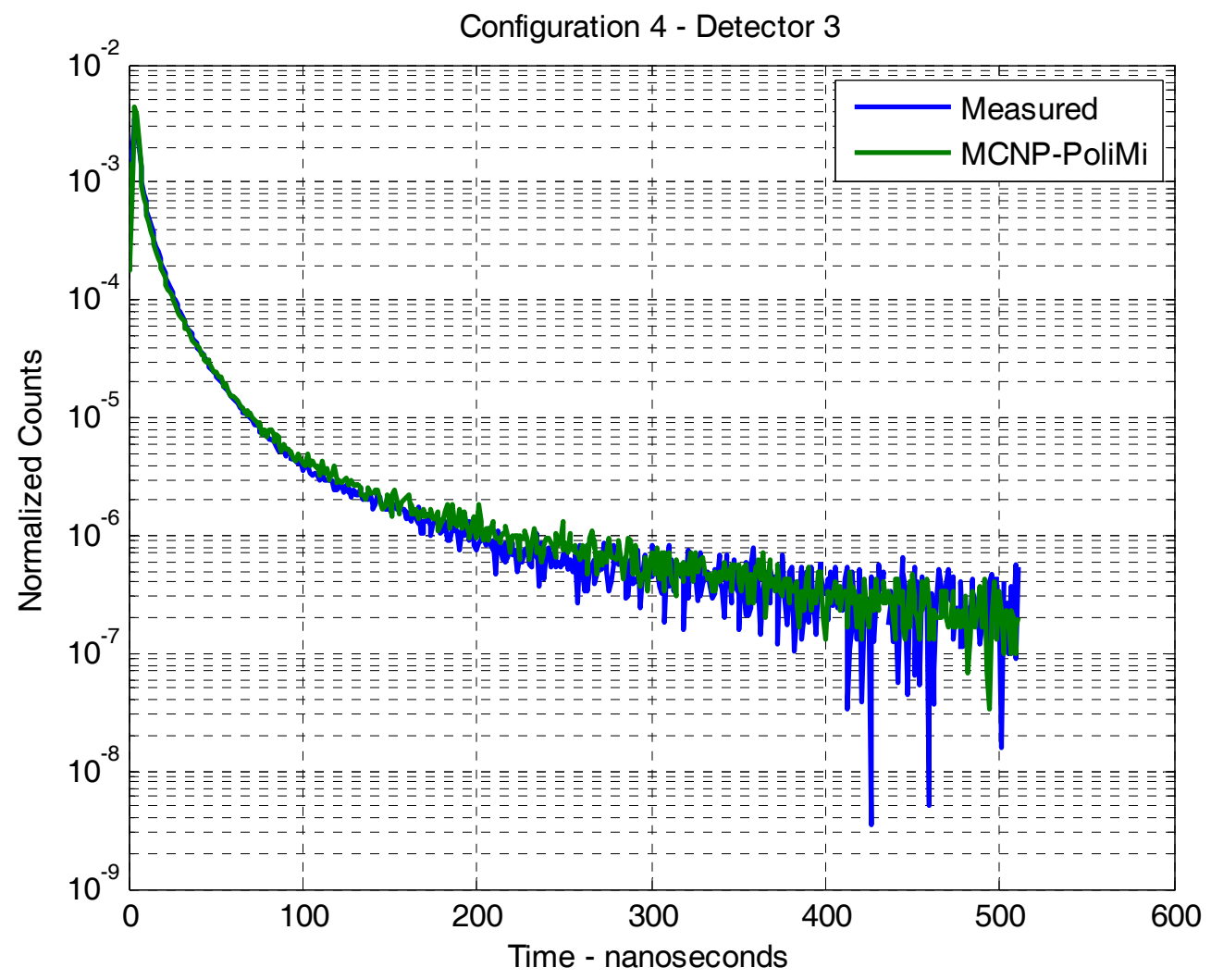

Fig. E.32. Time distribution of counts in detector 3 after Cf fission for 512 ns for configuration 3. 


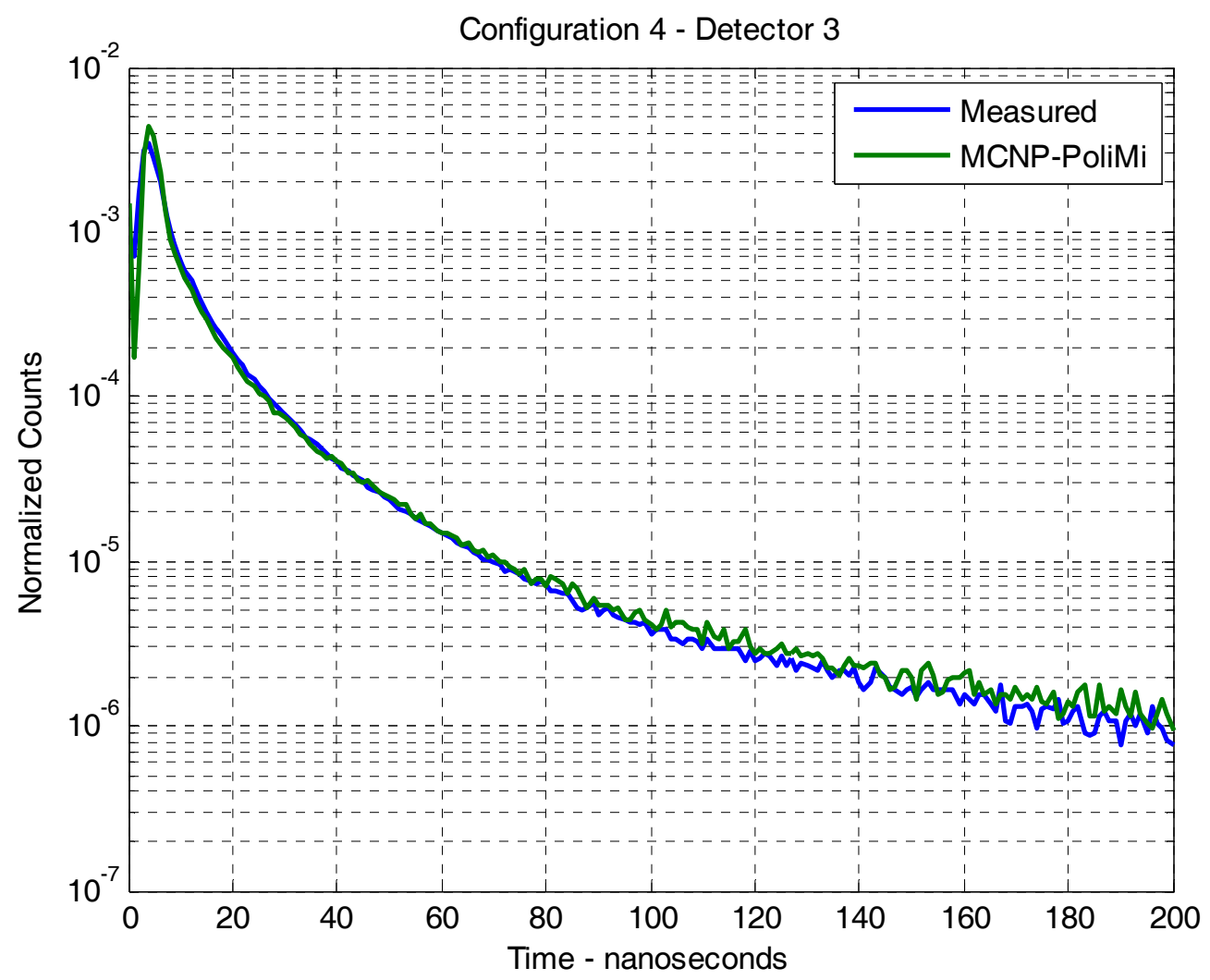

Fig. E.33. Time distribution of counts in detector 3 after $\mathbf{C f}$ fission for $200 \mathrm{~ns}$ for configuration 4. 


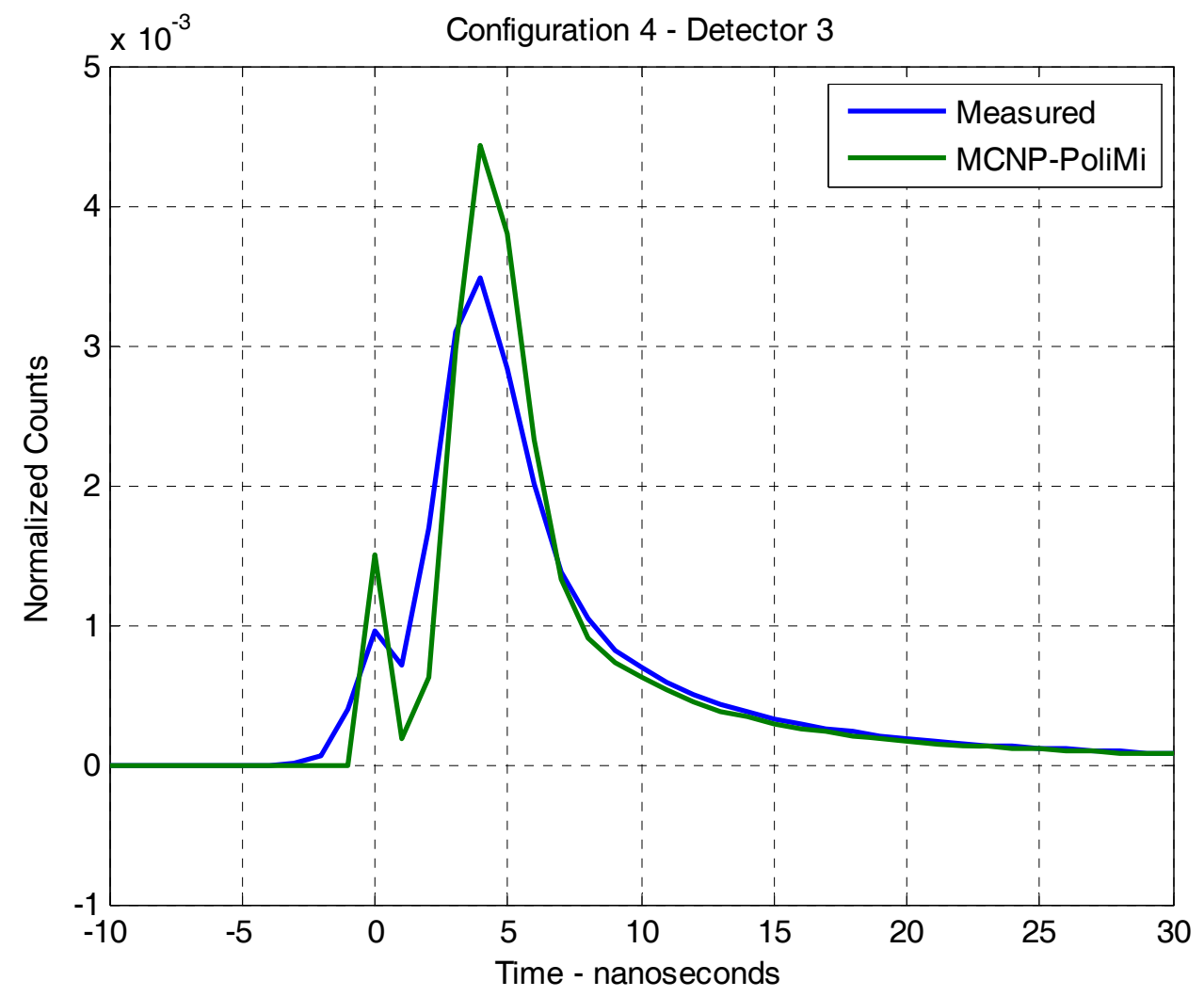

Fig. E.34. Time distribution of counts in detector 3 after $\mathbf{C f}$ fission for $\mathbf{3 0}$ ns for configuration 4. 


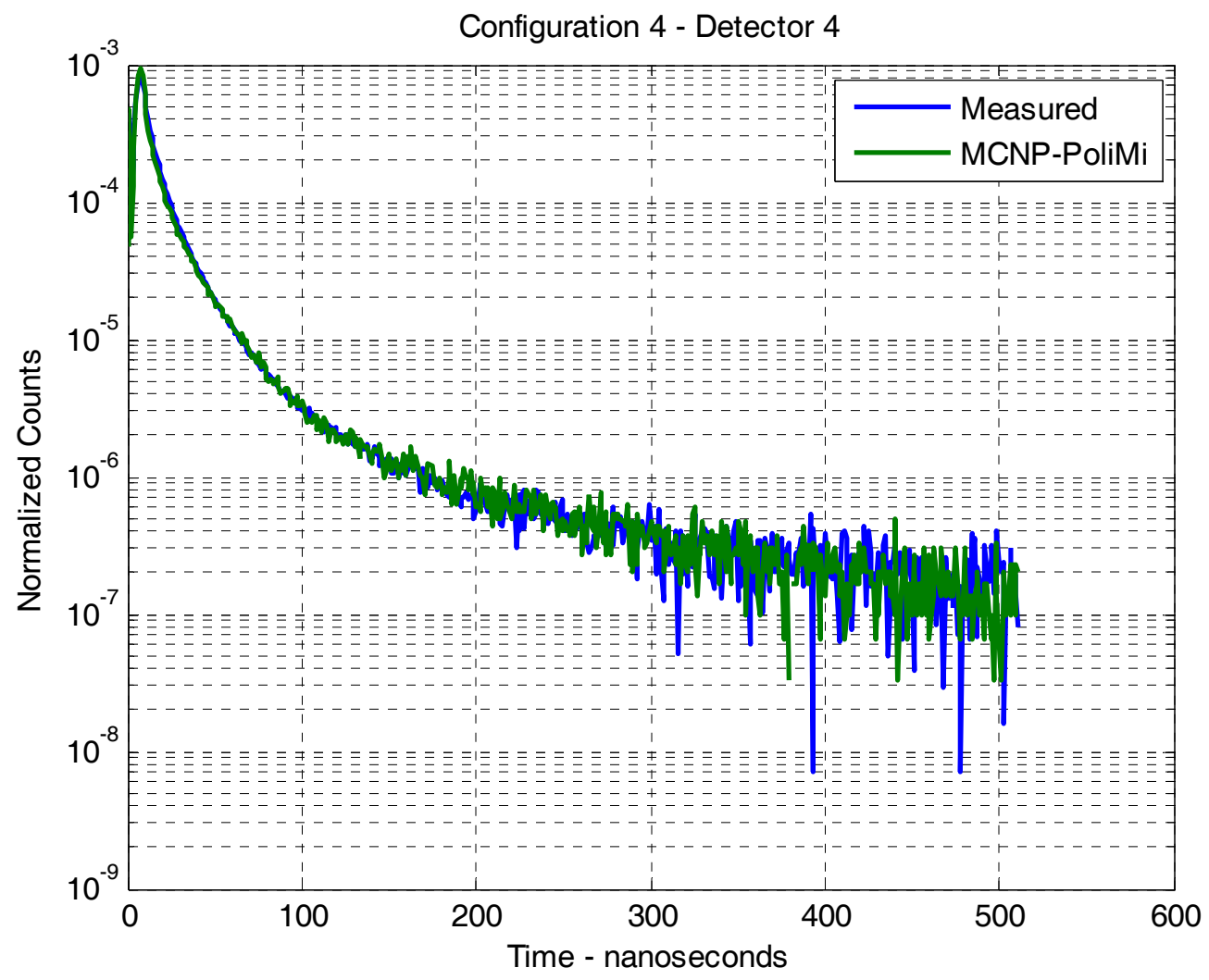

Fig. E.35. Time distribution of counts in detector 4 after Cf fission for 512 ns for configuration 4. 


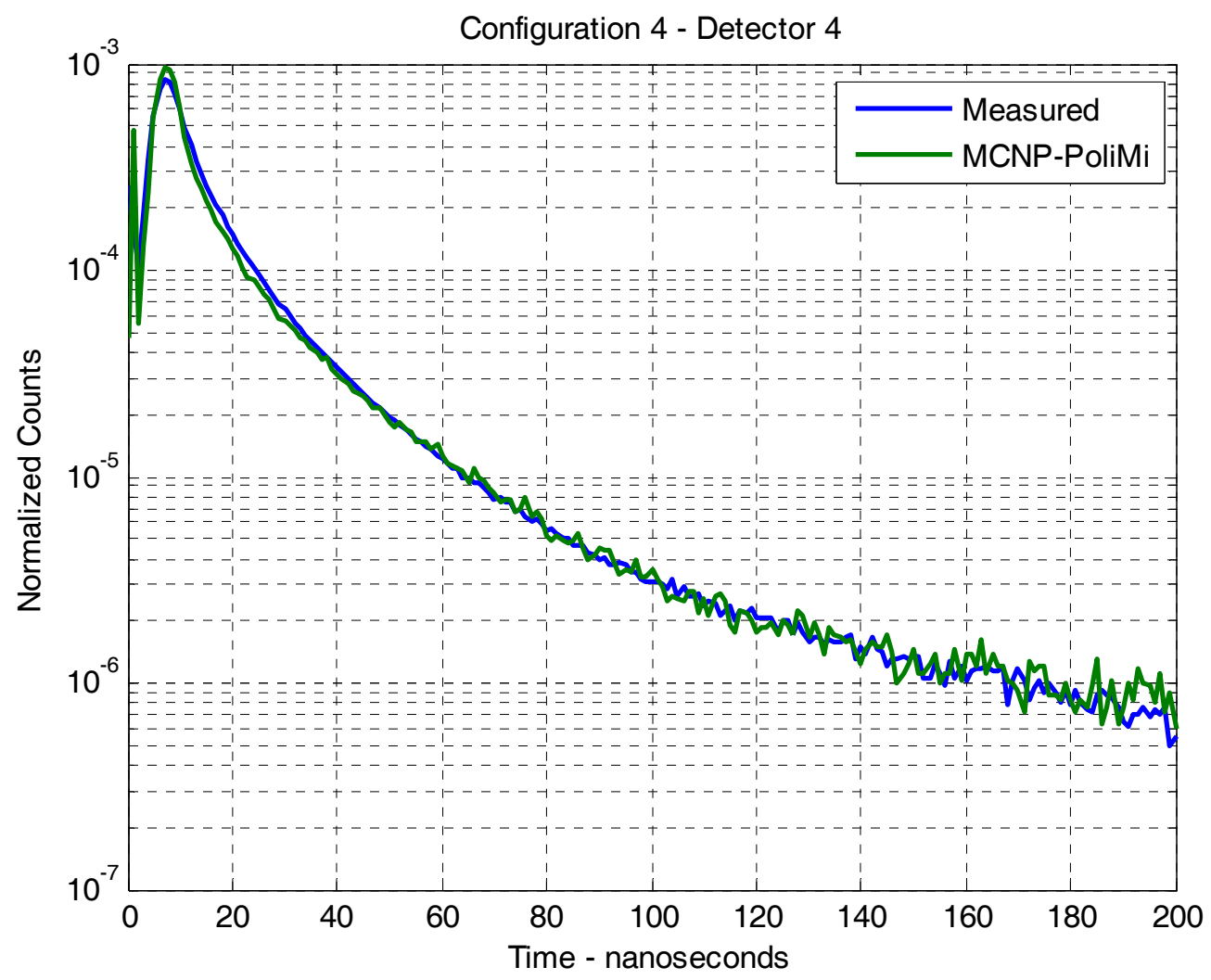

Fig. E.36. Time distribution of counts in detector 4 after $\mathbf{C f}$ fission for $200 \mathrm{~ns}$ for configuration 4. 


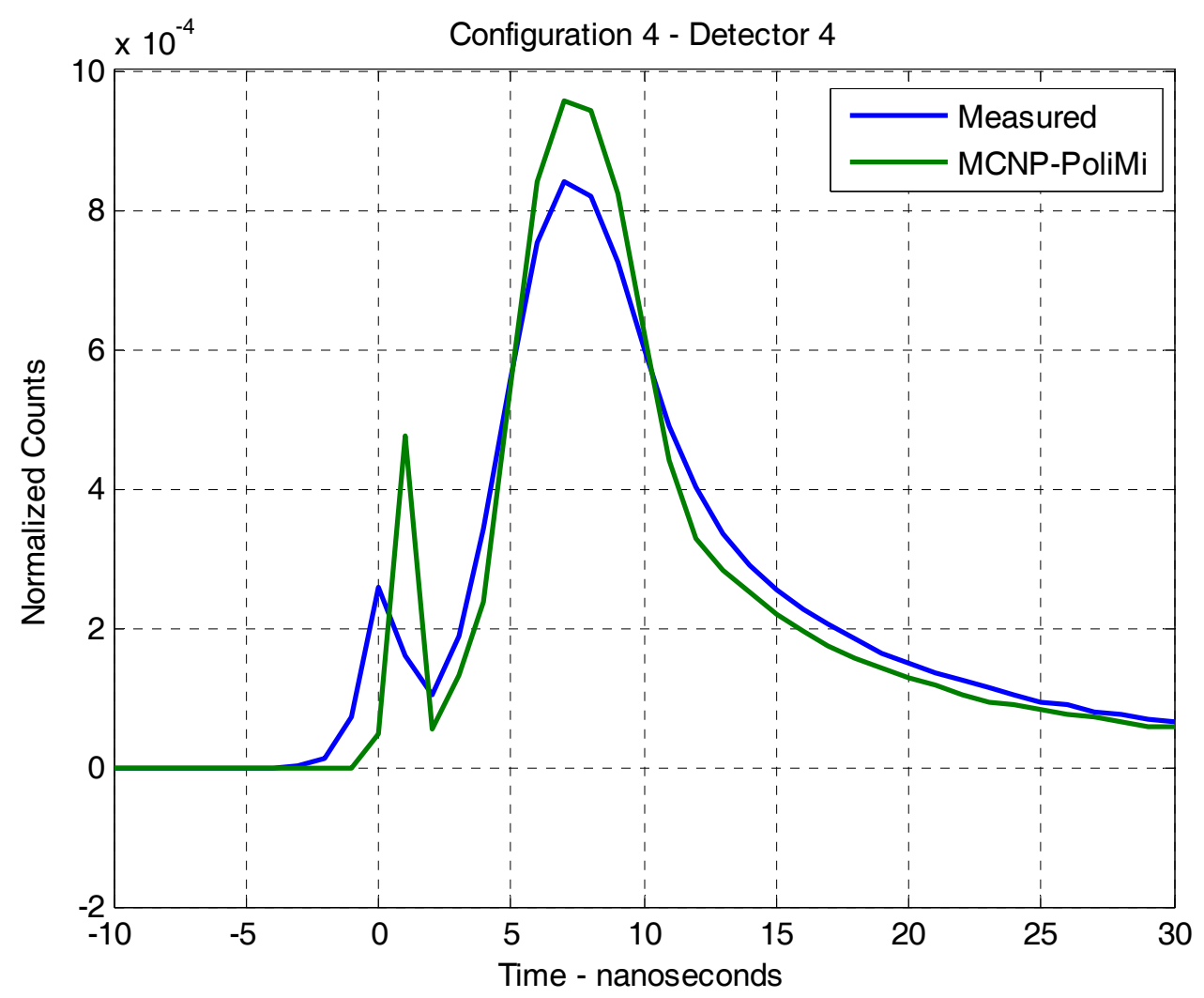

Fig. E.37. Time distribution of counts in detector 4 after $\mathbf{C f}$ fission for $\mathbf{3 0}$ ns for configuration 4. 


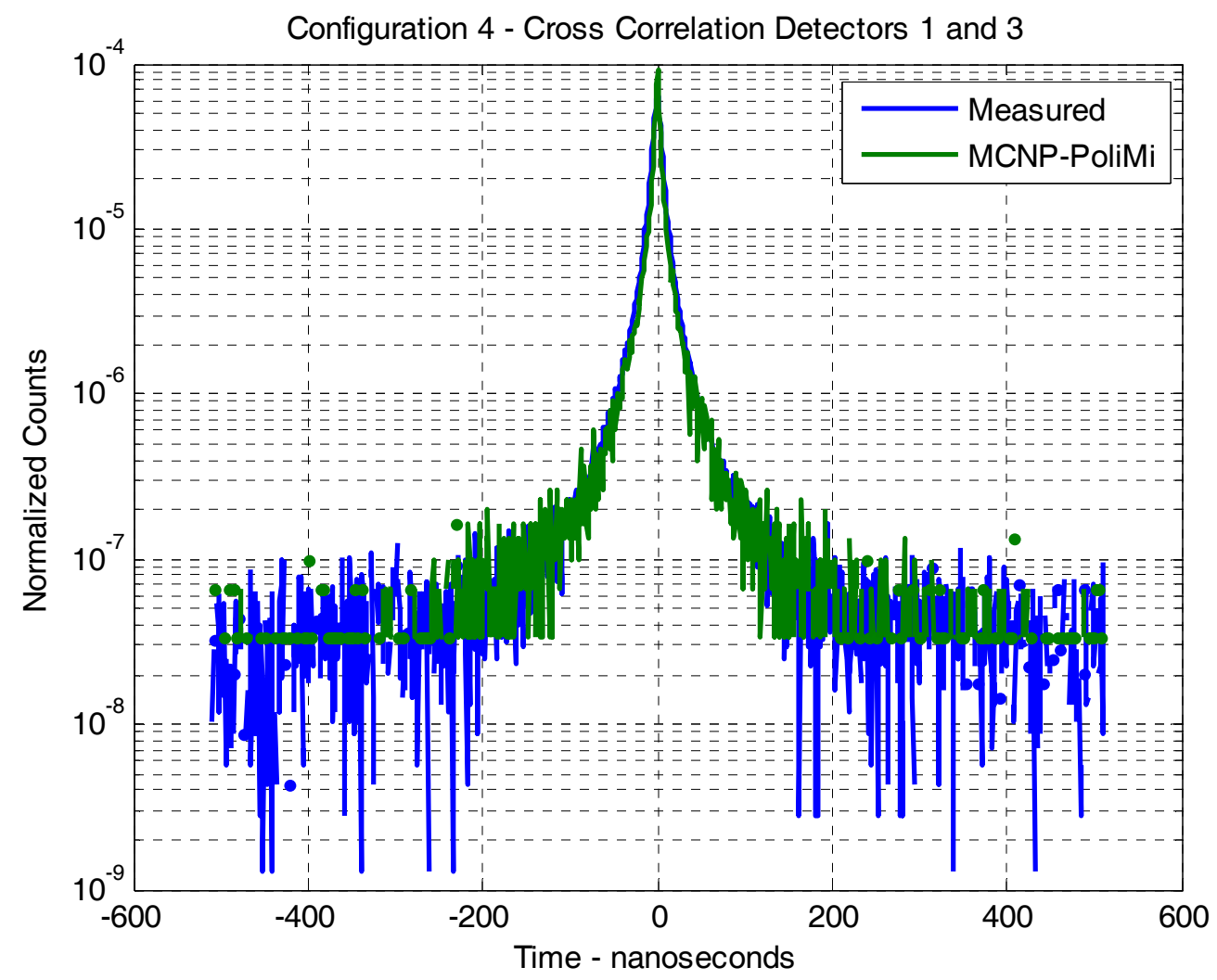

Fig. E.38. Time distribution of counts in detector 3 after a count in detector 1 for 512 ns for configuration 4. 


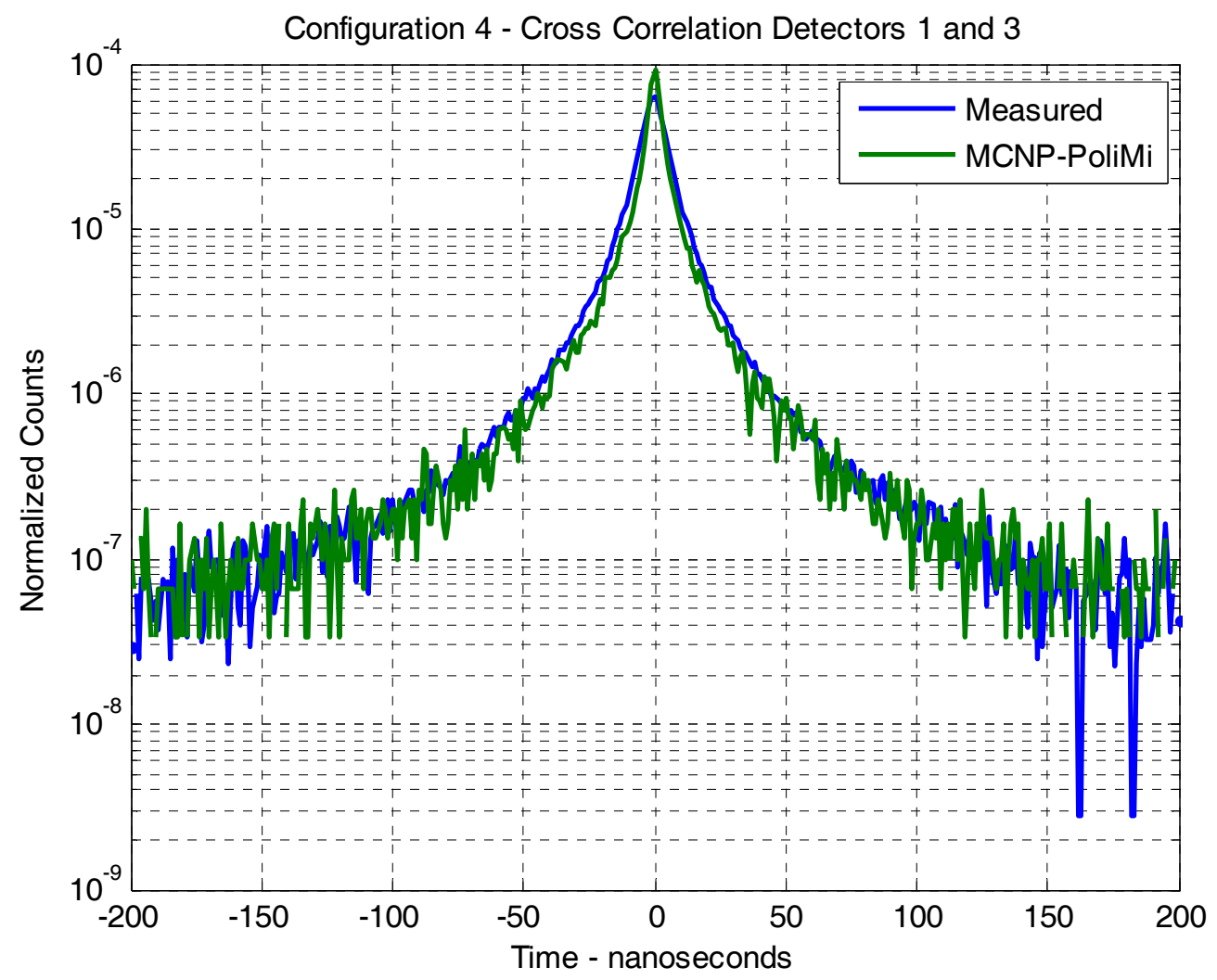

Fig. E.39. Time distribution of counts in detector 3 after a count in detector 1 for 200 ns for configuration 4. 


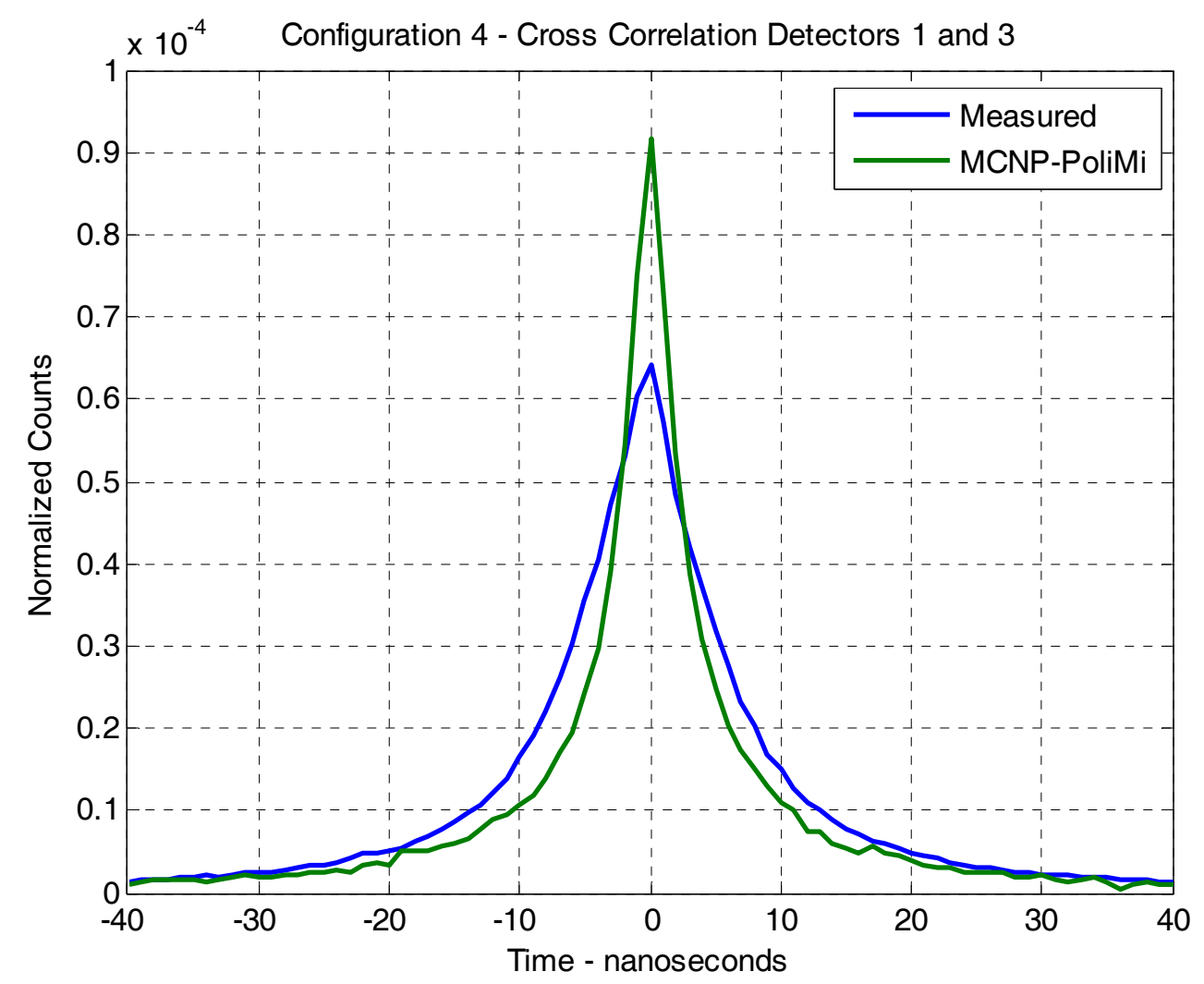

Fig. E.40. Time distribution of counts in detector 3 after a count in detector 1 for $\mathbf{4 0}$ ns for configuration 4. 
Configuration 12:
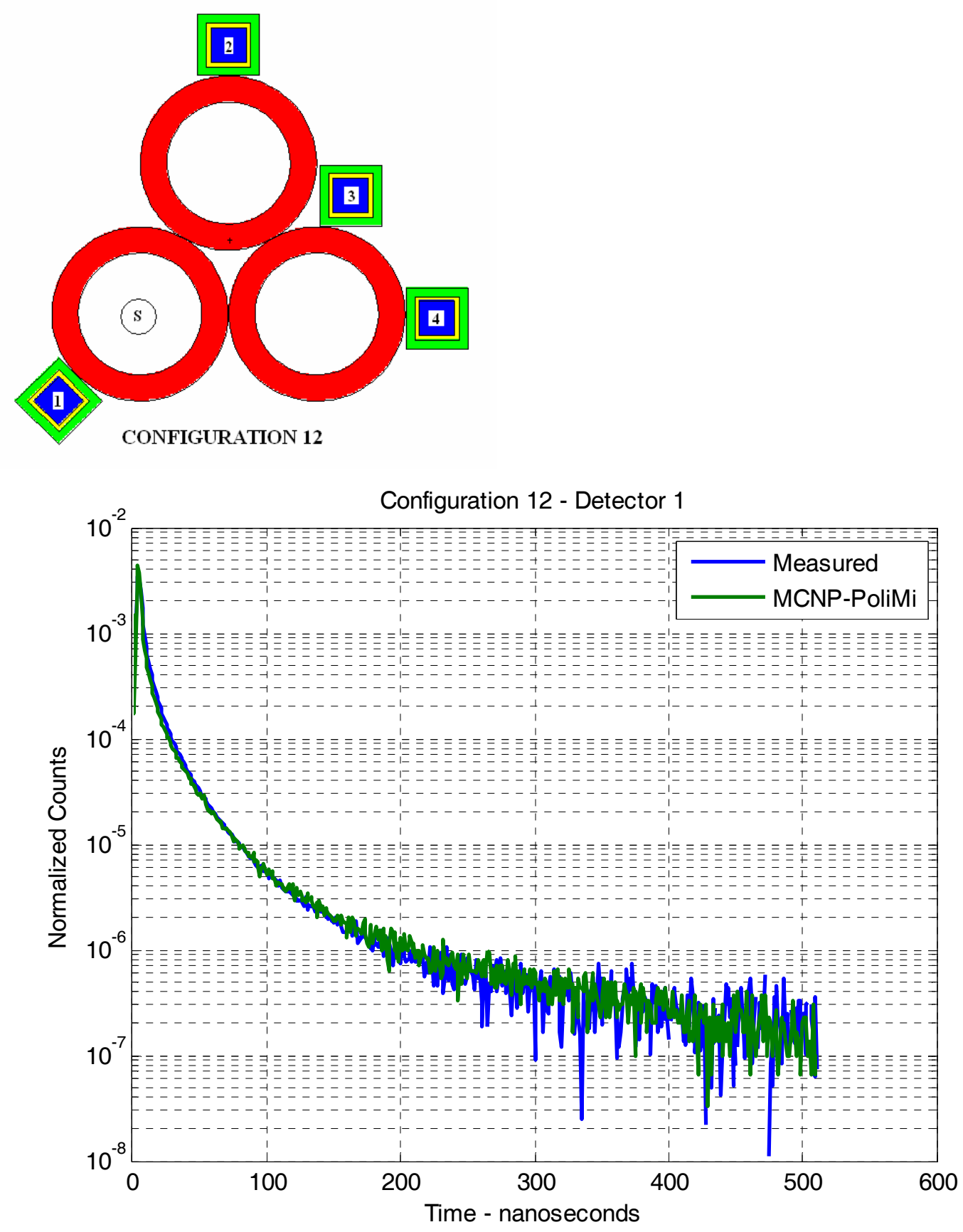

Fig. E.41. Time distribution of counts in detector 1 after $\mathbf{C f}$ fission for $\mathbf{5 1 2} \mathrm{ns}$ for configuration 12. 


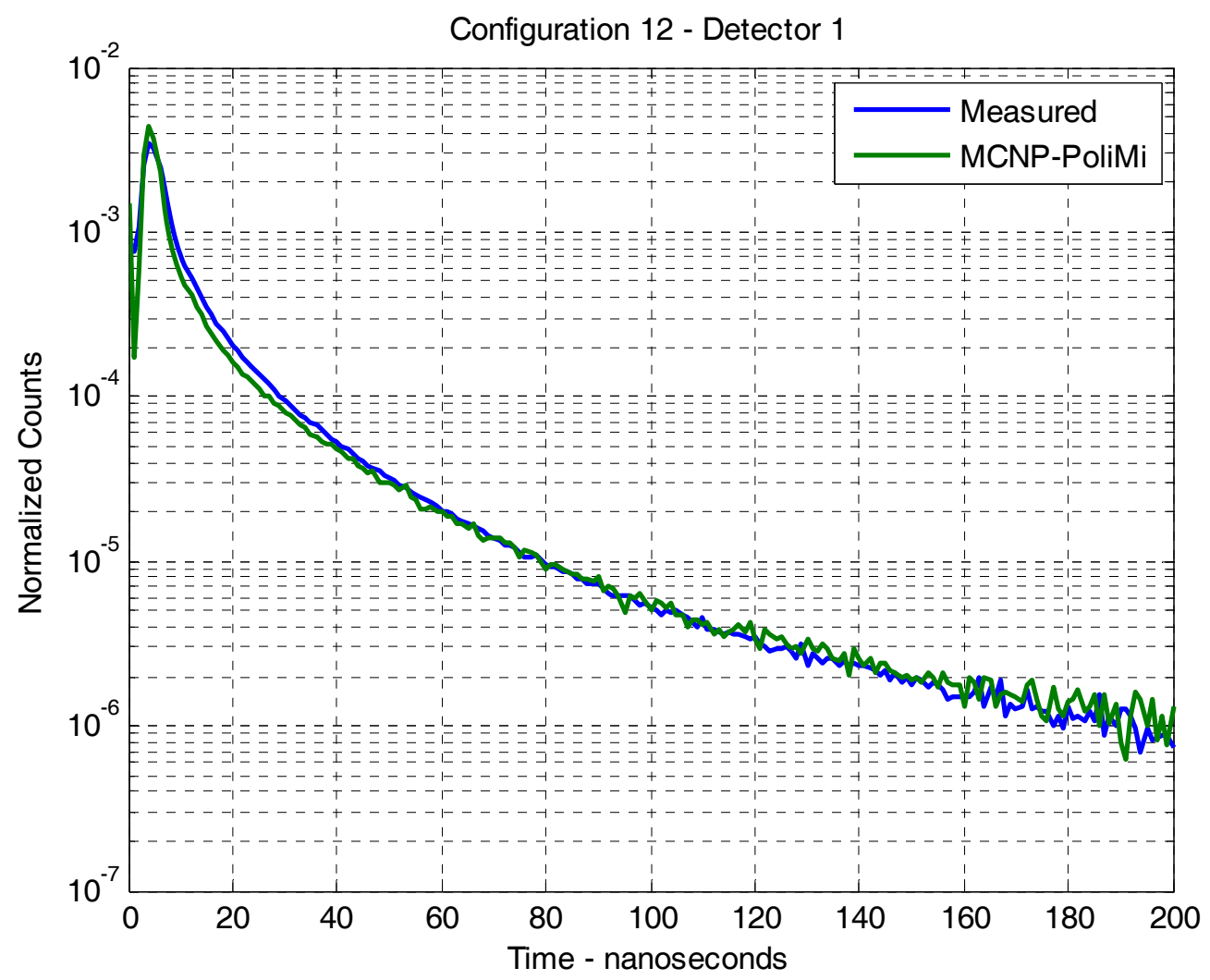

Fig. E.42. Time distribution of counts in detector $\mathbf{1}$ after $\mathbf{C f}$ fission for $200 \mathrm{~ns}$ for configuration 12. 


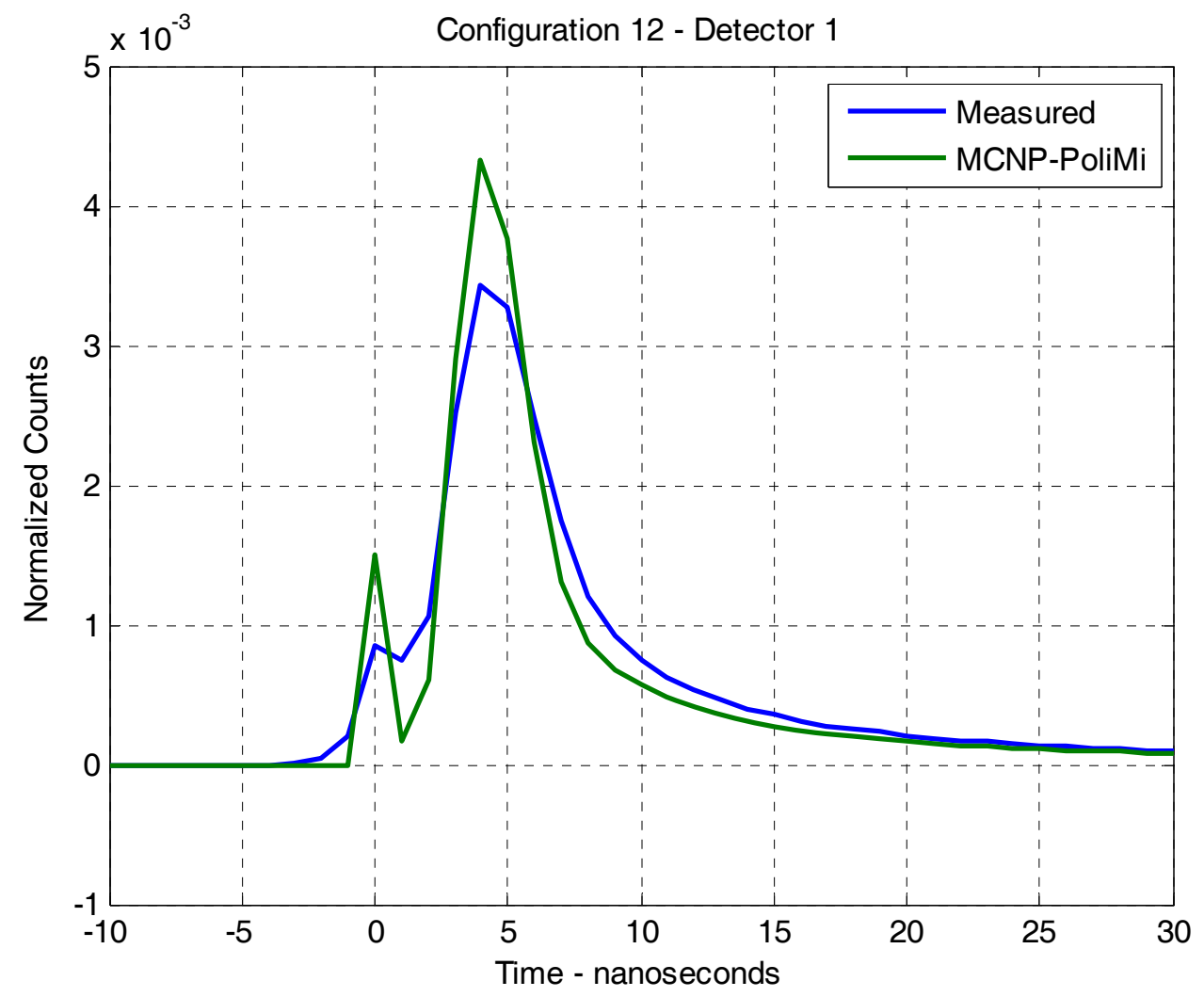

Fig. E.43. Time distribution of counts in detector 1 after $\mathbf{C f}$ fission for $30 \mathrm{~ns}$ for configuration 12. 


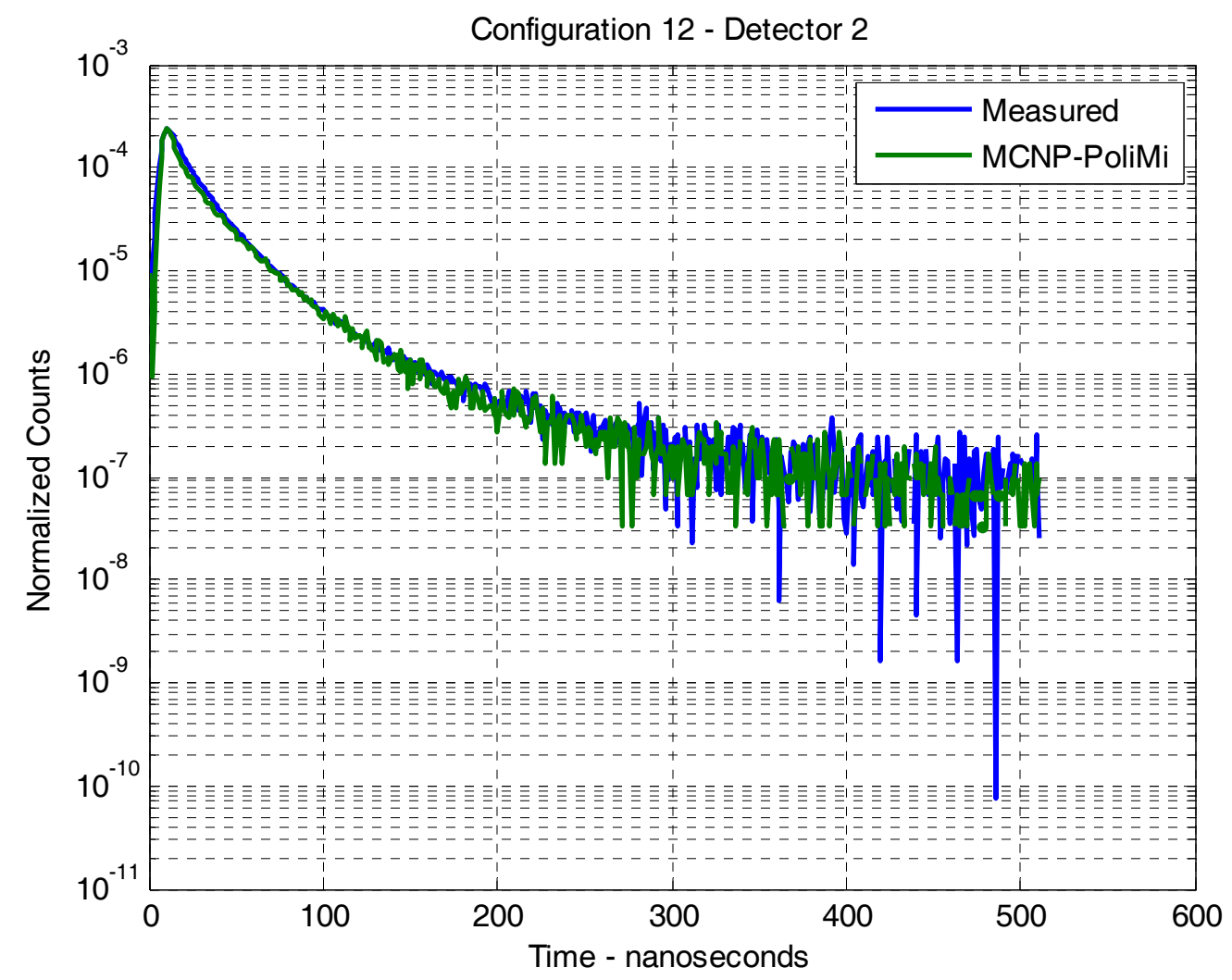

Fig. E.44. Time distribution of counts in detector $\mathbf{2}$ after $\mathbf{C f}$ fission for $\mathbf{5 1 2}$ ns for configuration 12. 


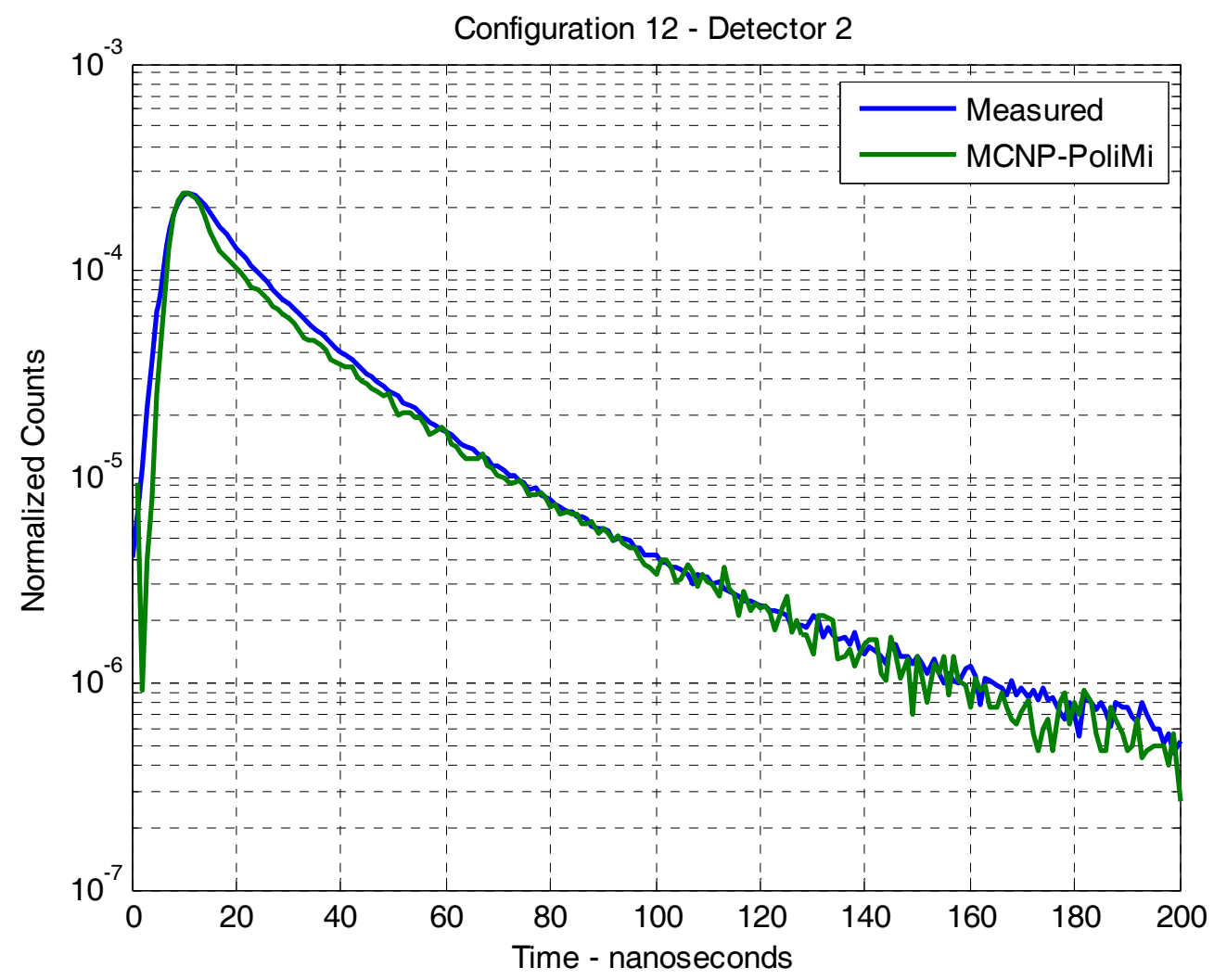

Fig. E.45. Time distribution of counts in detector 2 after $\mathbf{C f}$ fission for $200 \mathrm{~ns}$ for configuration 12. 


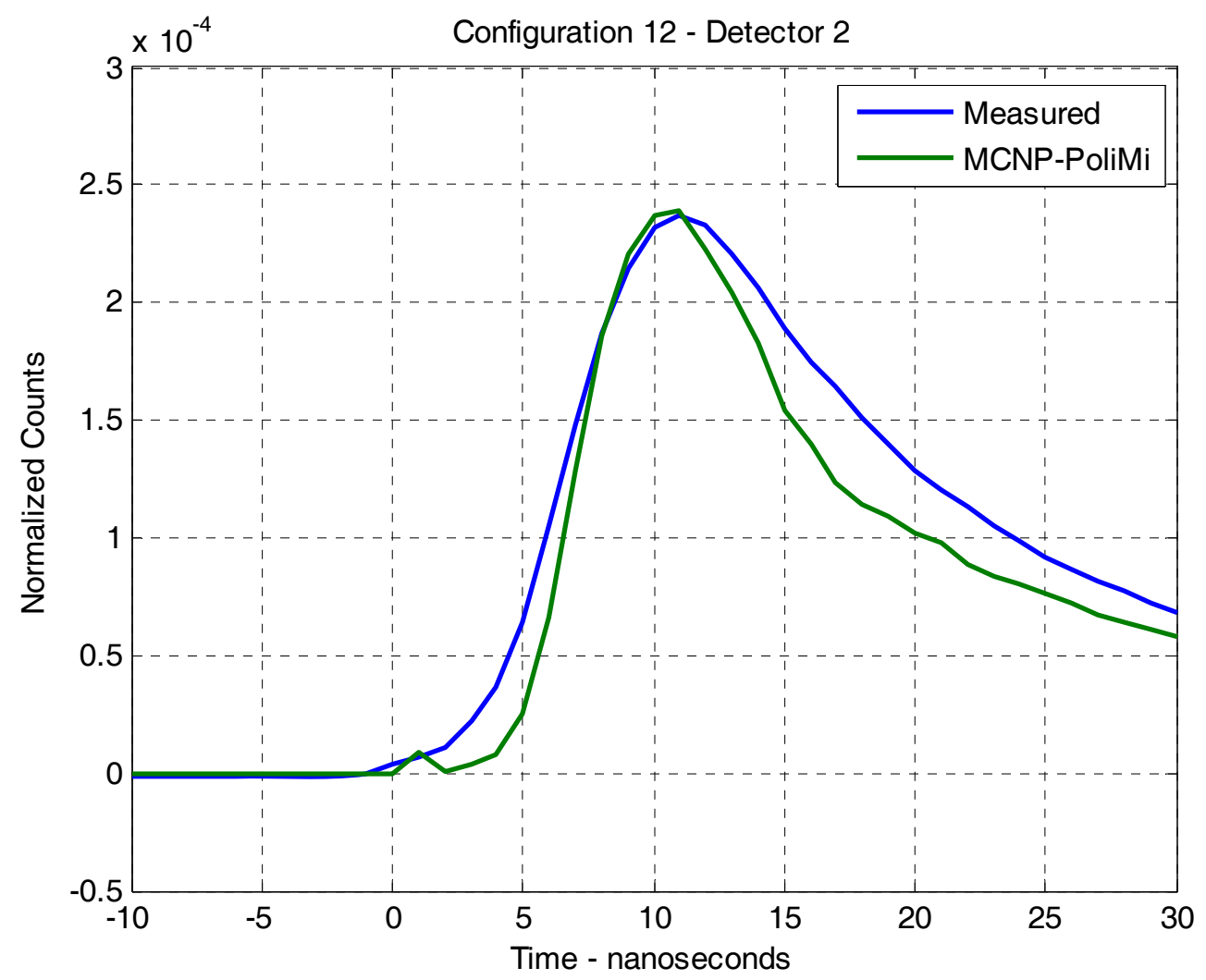

Fig. E.46. Time distribution of counts in detector 2 after $\mathbf{C f}$ fission for $30 \mathrm{~ns}$ for configuration 12. 


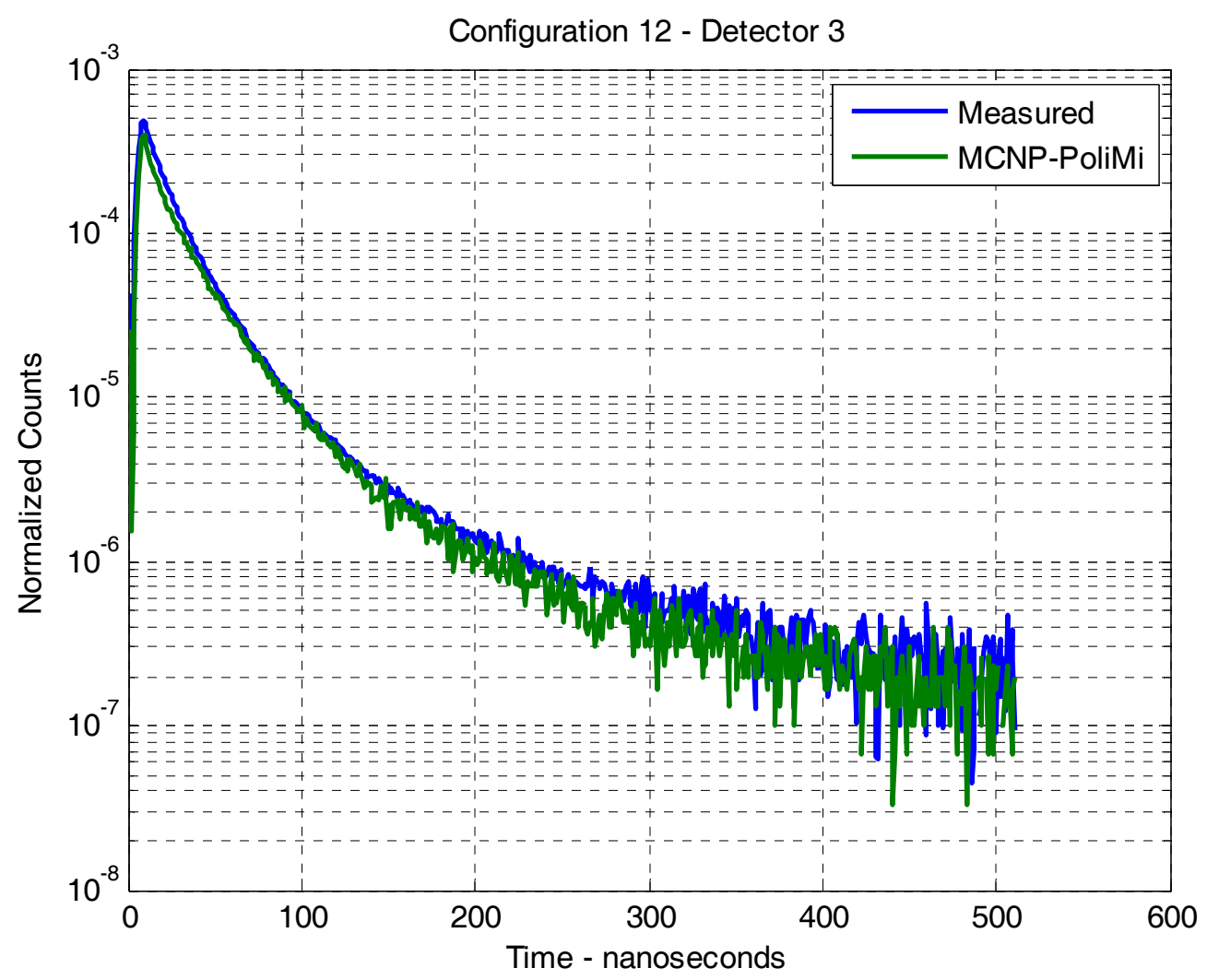

Fig. E.47. Time distribution of counts in detector 3 after $\mathbf{C f}$ fission for $\mathbf{5 1 2}$ ns for configuration 12. 


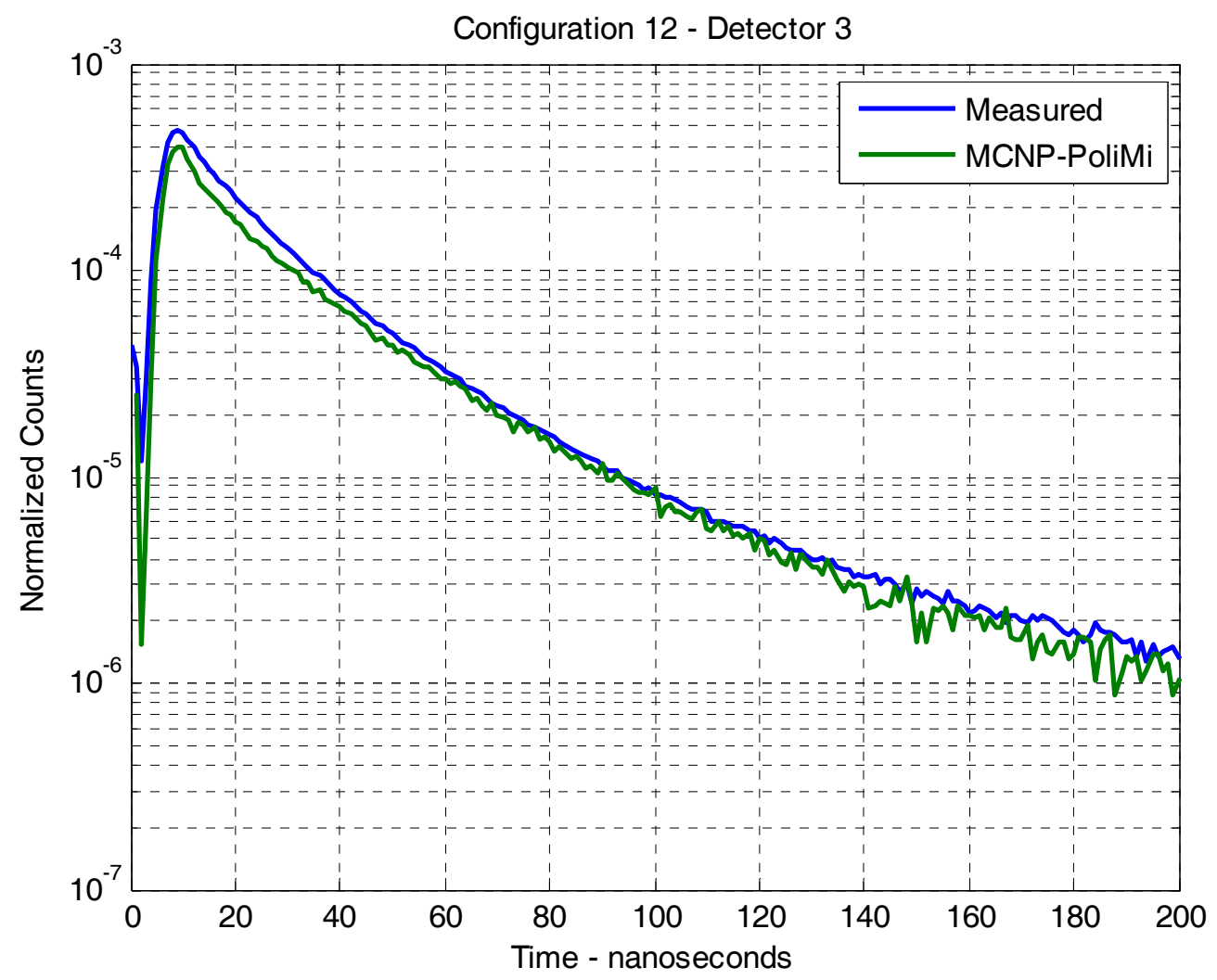

Fig. E.48. Time distribution of counts in detector 3 after $\mathbf{C f}$ fission for $200 \mathrm{~ns}$ for configuration 12. 


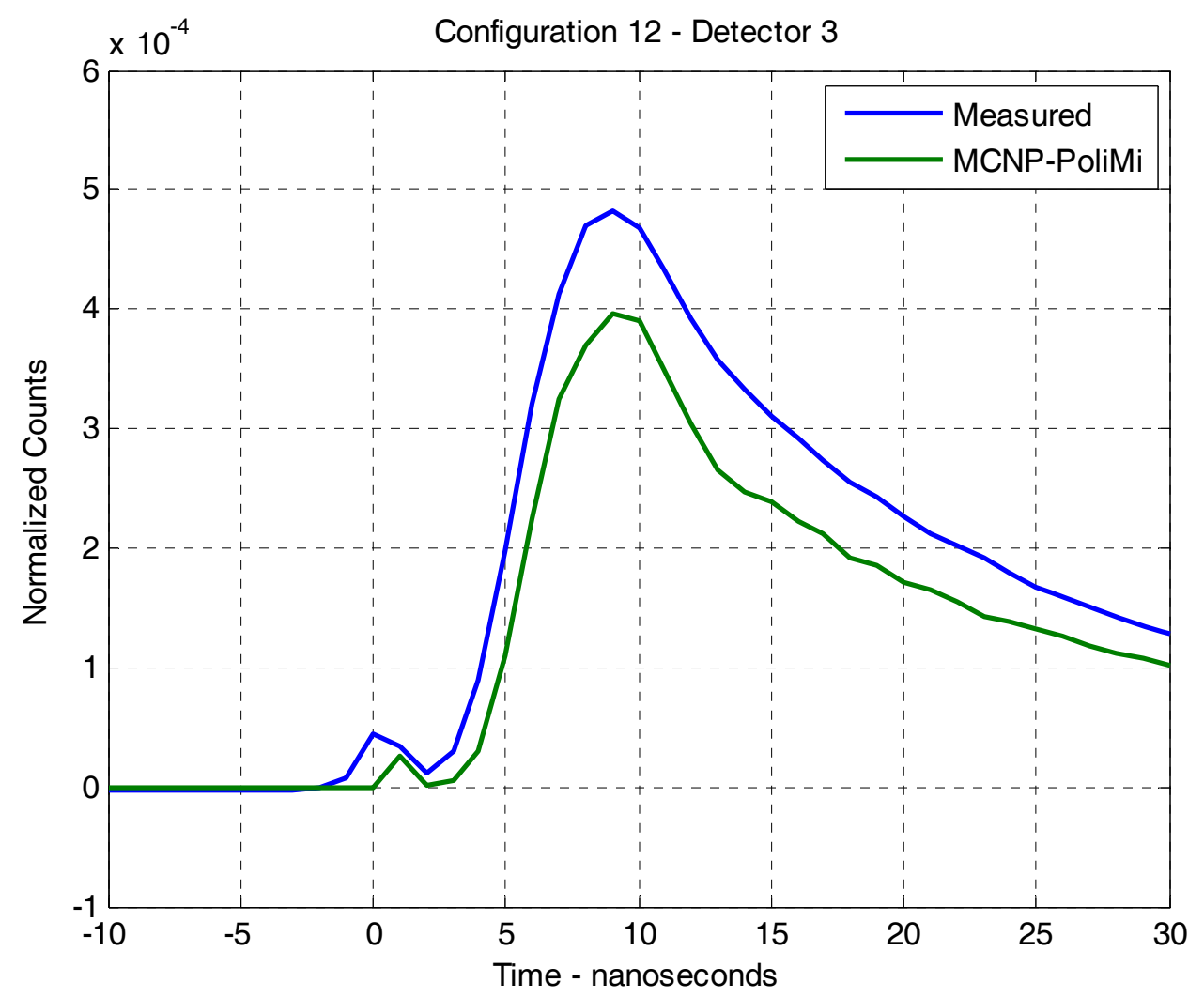

Fig. E.49. Time distribution of counts in detector 3 after $\mathbf{C f}$ fission for $30 \mathrm{~ns}$ for configuration 12. 


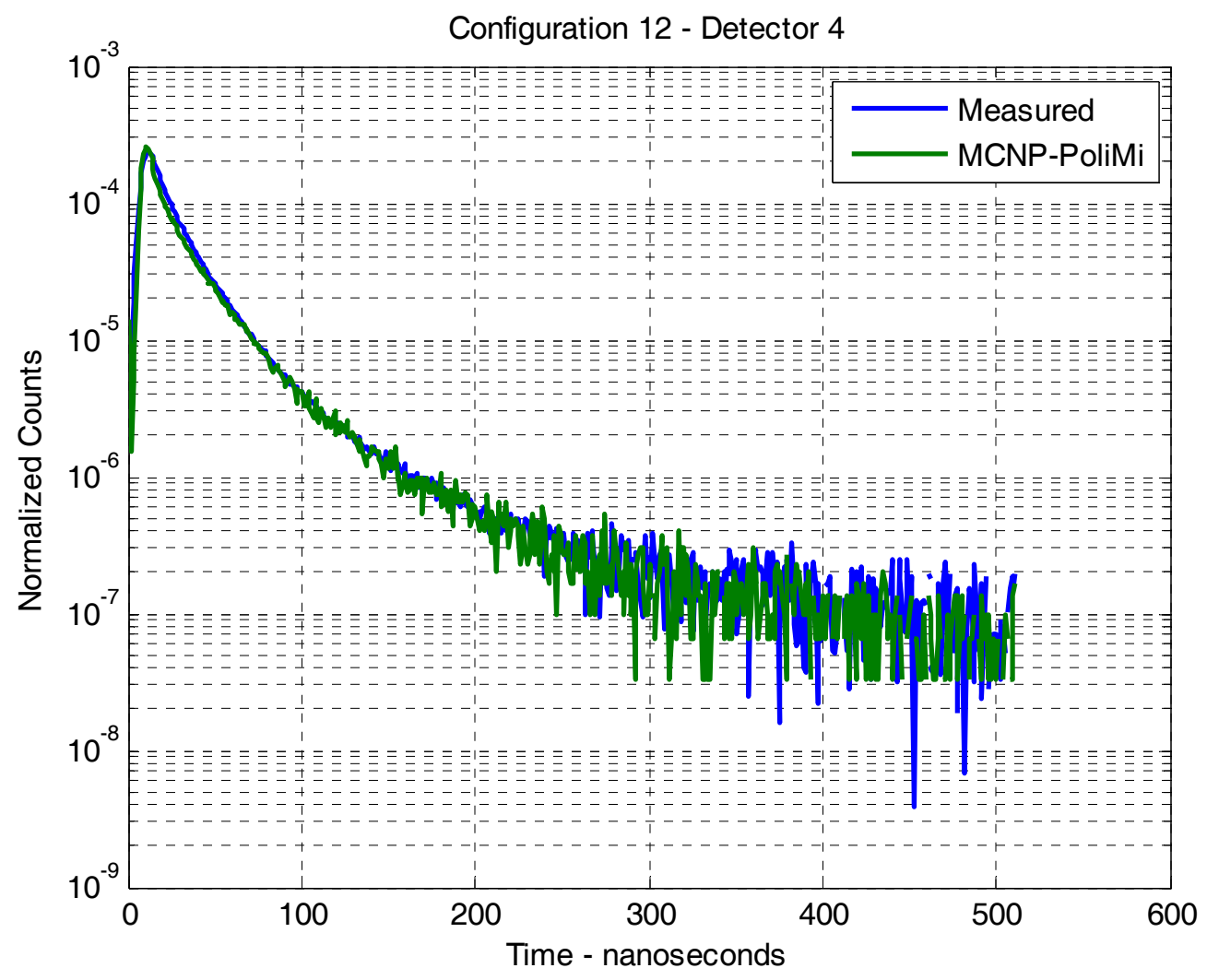

Fig. E.50. Time distribution of counts in detector $\mathbf{4}$ after $\mathbf{C f}$ fission for $\mathbf{5 1 2}$ ns for configuration 12. 


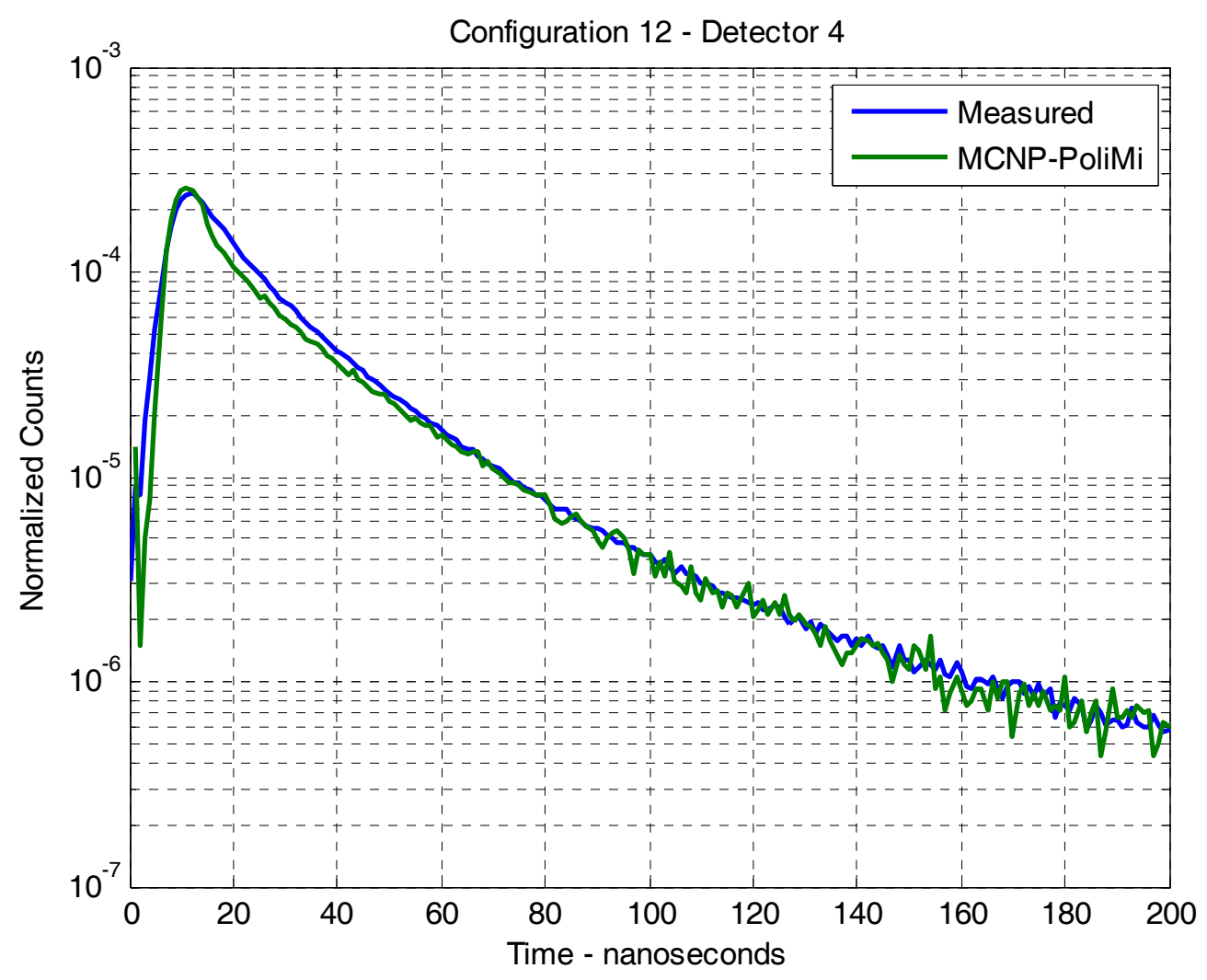

Fig. E.51. Time distribution of counts in detector 4 after $\mathbf{C f}$ fission for $200 \mathrm{~ns}$ for configuration 12. 


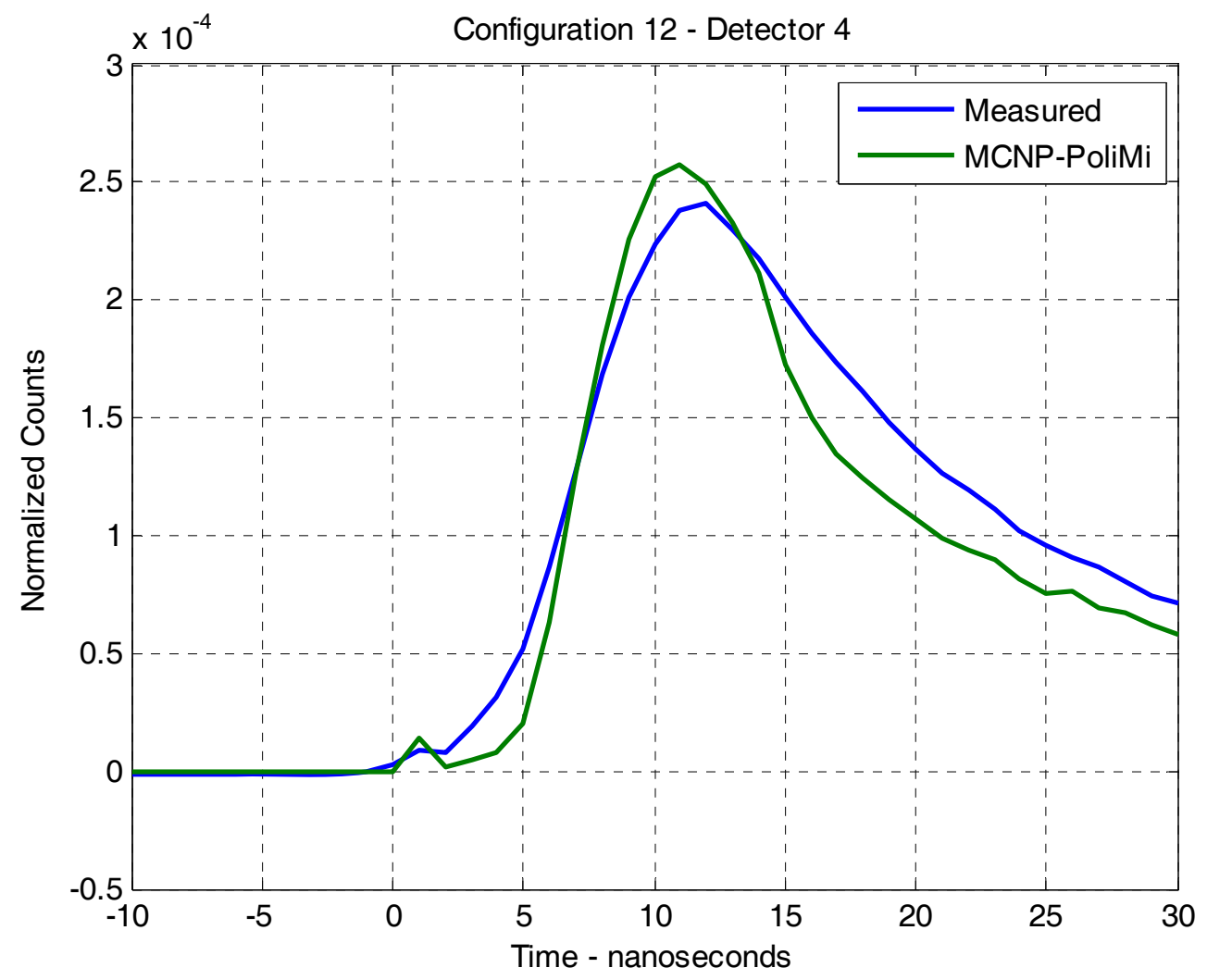

Fig. E.52. Time distribution of counts in detector 4 after $\mathbf{C f}$ fission for $\mathbf{3 0}$ ns for configuration 12. 


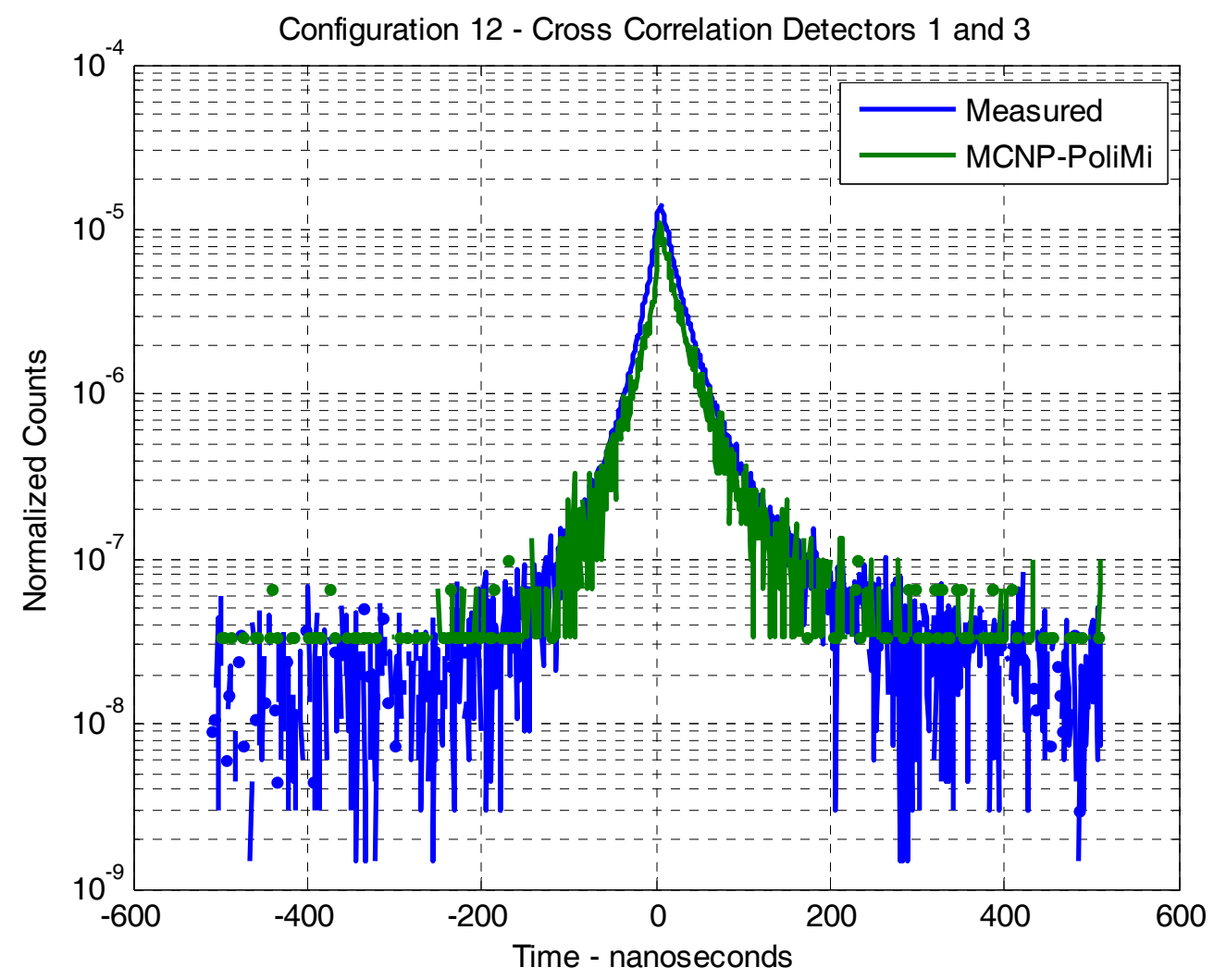

Fig. E.53. Time distribution of counts in detector 3 after a count in detector 1 for 512 ns for configuration 12. 


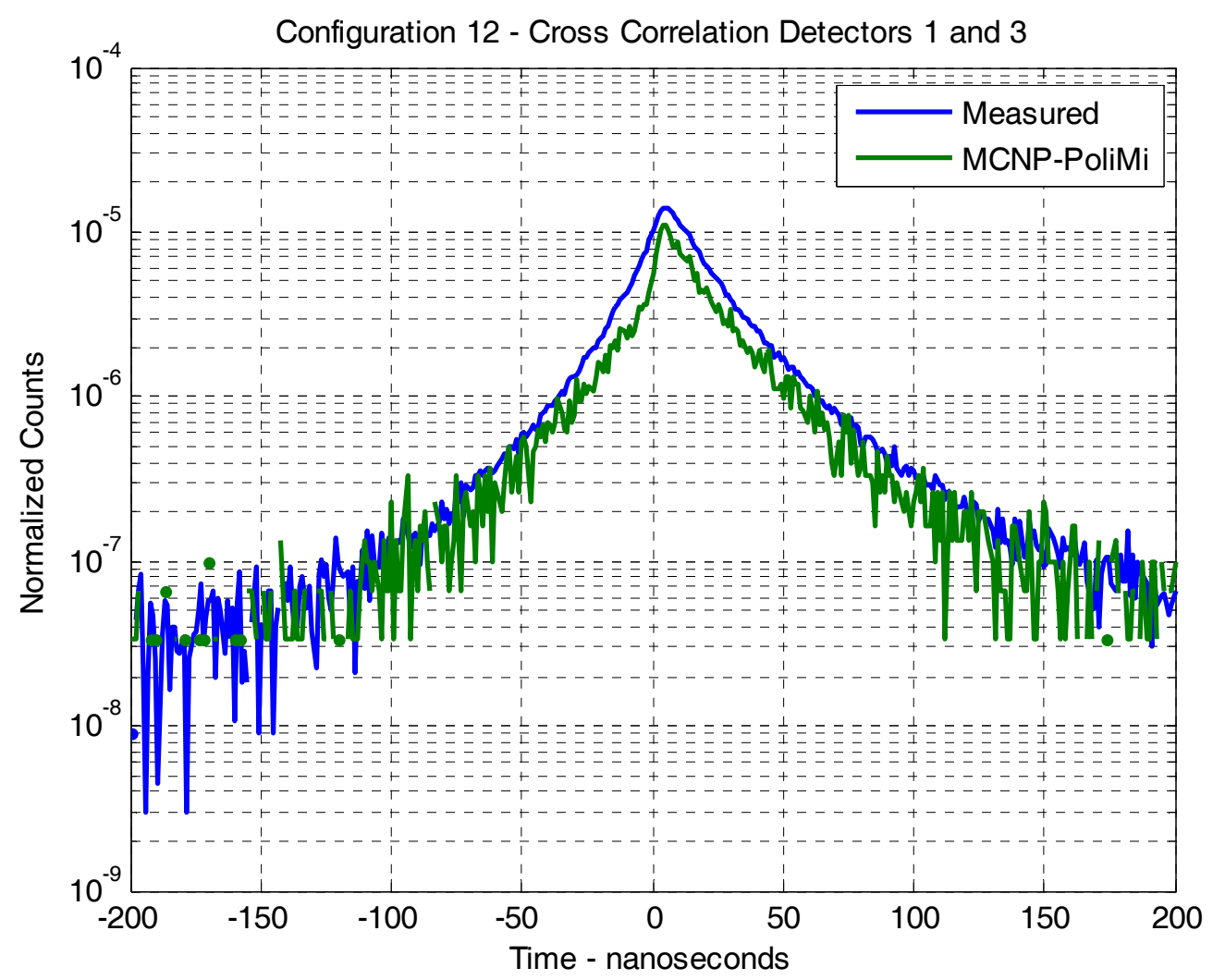

Fig. E.54. Time distribution of counts in detector 3 after a count in detector 1 for 200 ns for configuration 12. 


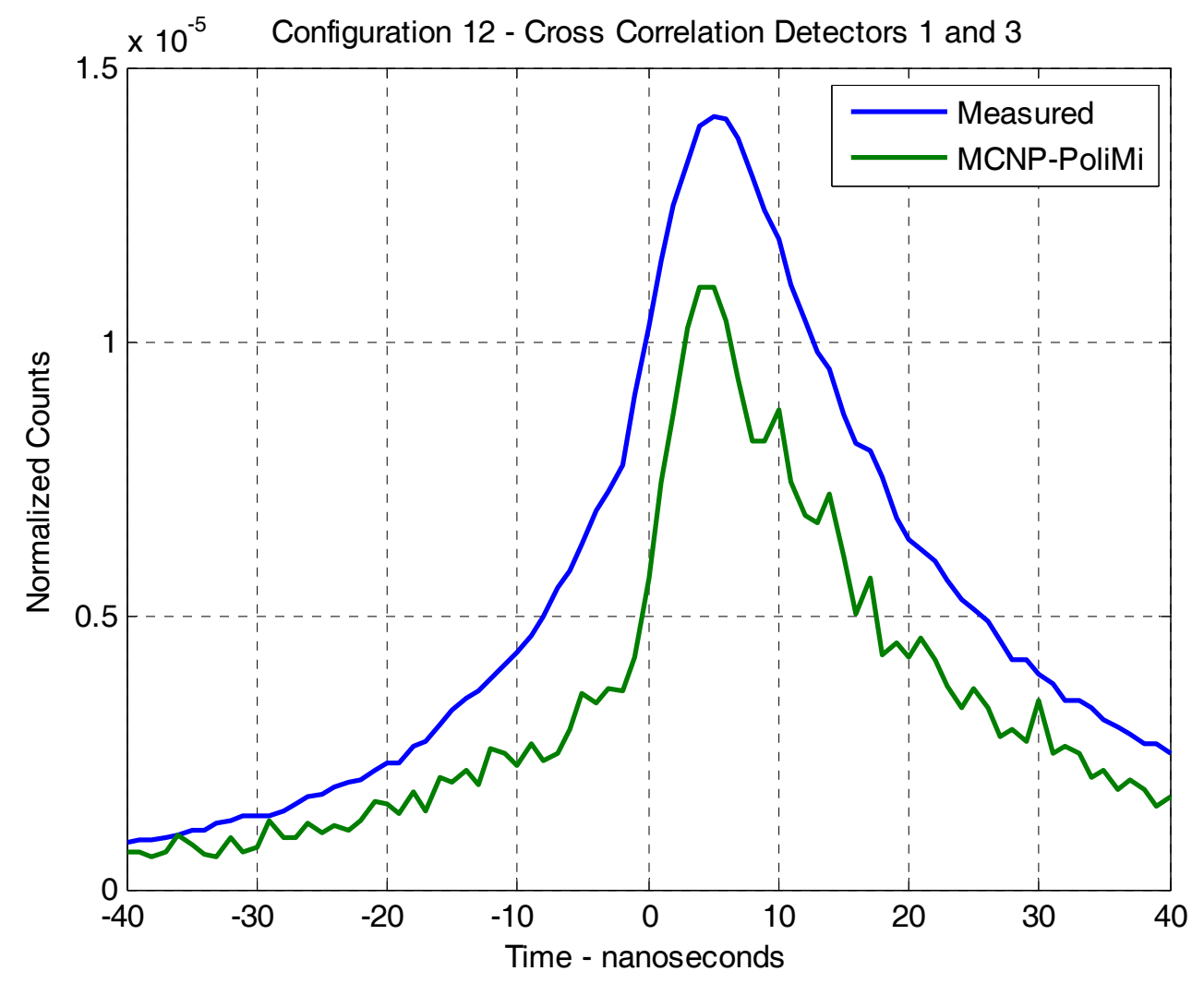

Fig. E.55. Time distribution of counts in detector 3 after a count in detector 1 for $\mathbf{4 0}$ ns for configuration 12. 
Configuration 20:
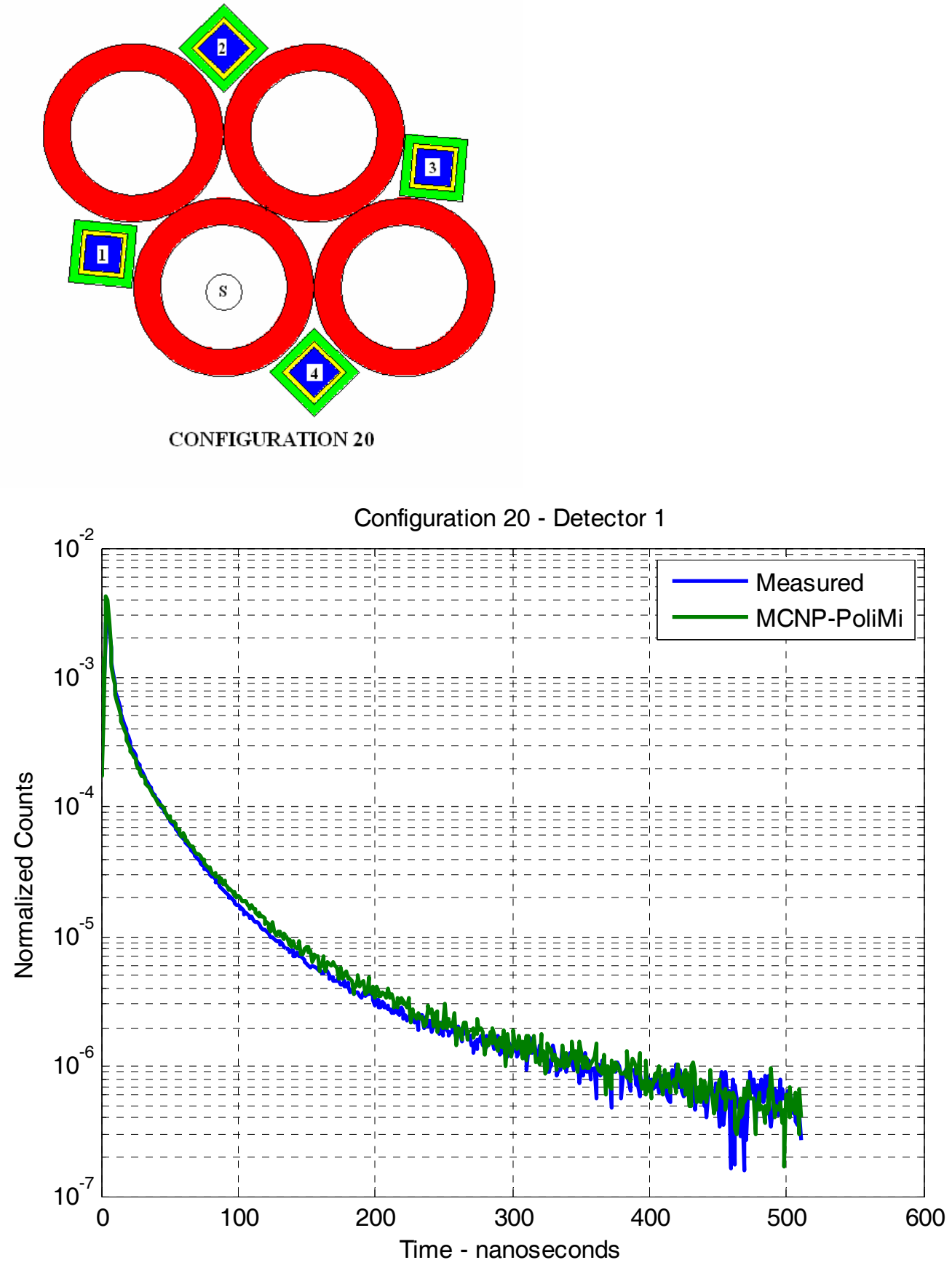

Fig. E.56. Time distribution of counts in detector 1 after $\mathbf{C f}$ fission for $\mathbf{5 1 2} \mathbf{n s}$ for configuration 20. 


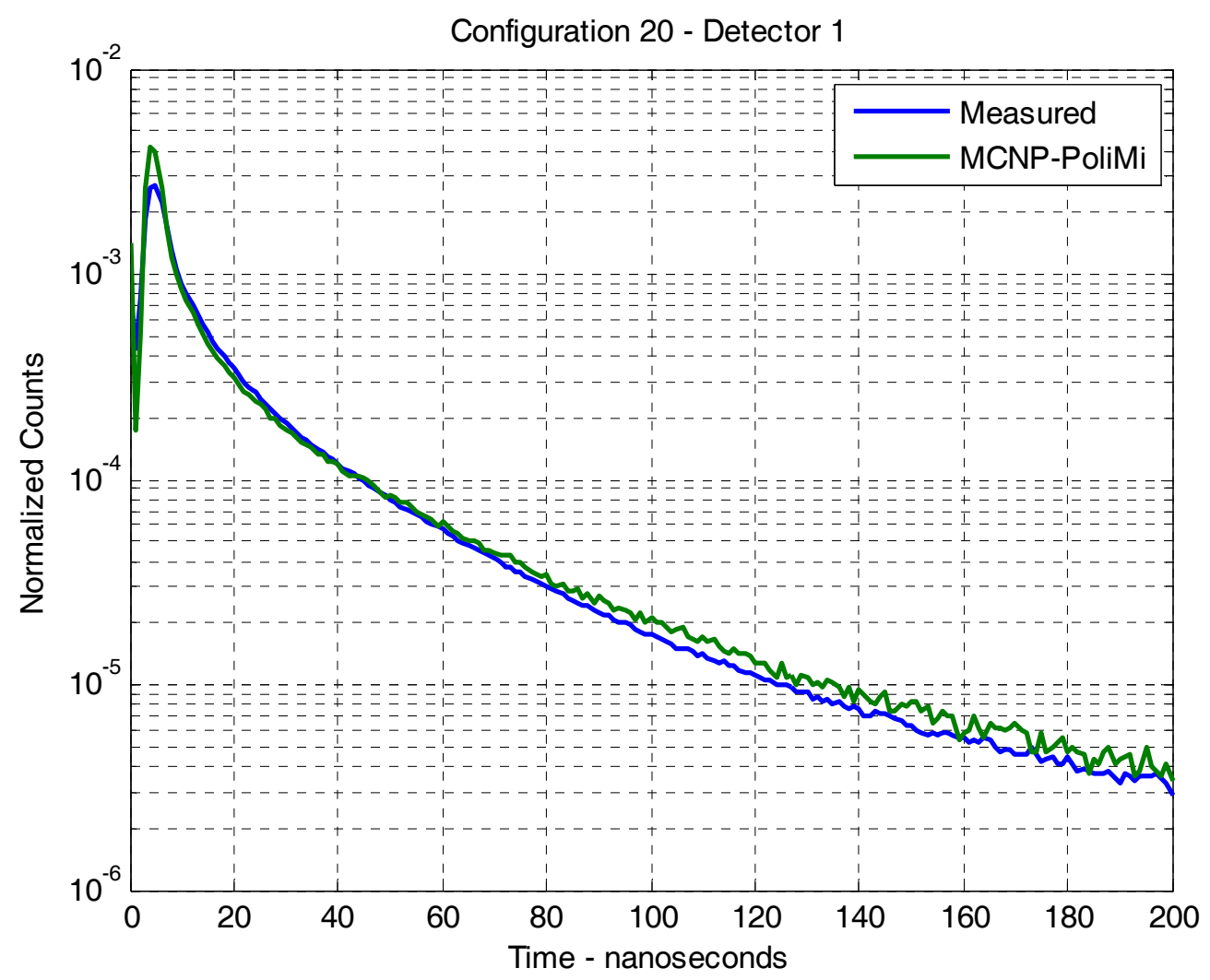

Fig. E.57. Time distribution of counts in detector $\mathbf{1}$ after $\mathbf{C f}$ fission for $200 \mathrm{~ns}$ for configuration 20. 


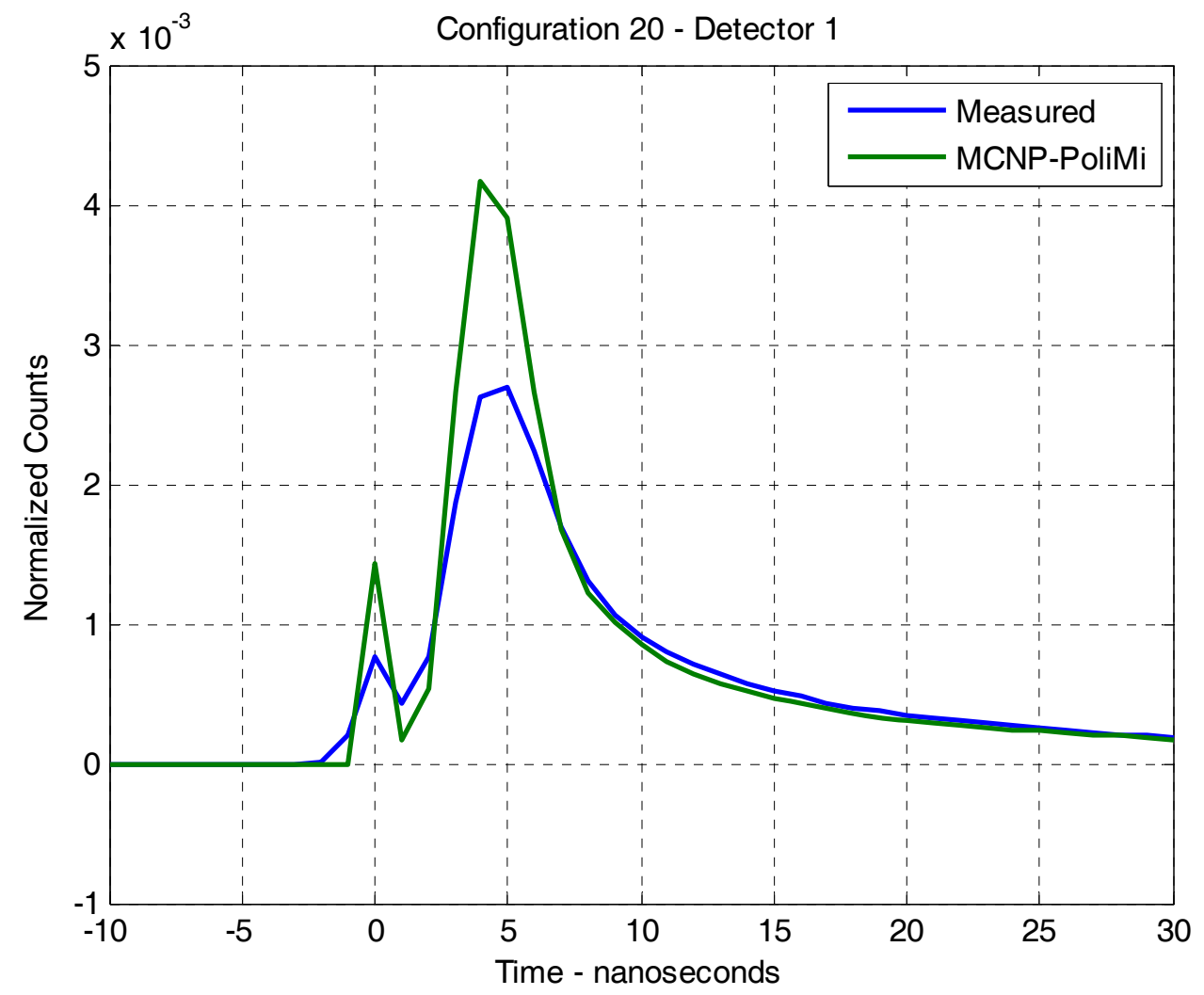

Fig. E.58. Time distribution of counts in detector 1 after $\mathbf{C f}$ fission for $30 \mathrm{~ns}$ for configuration 20. 


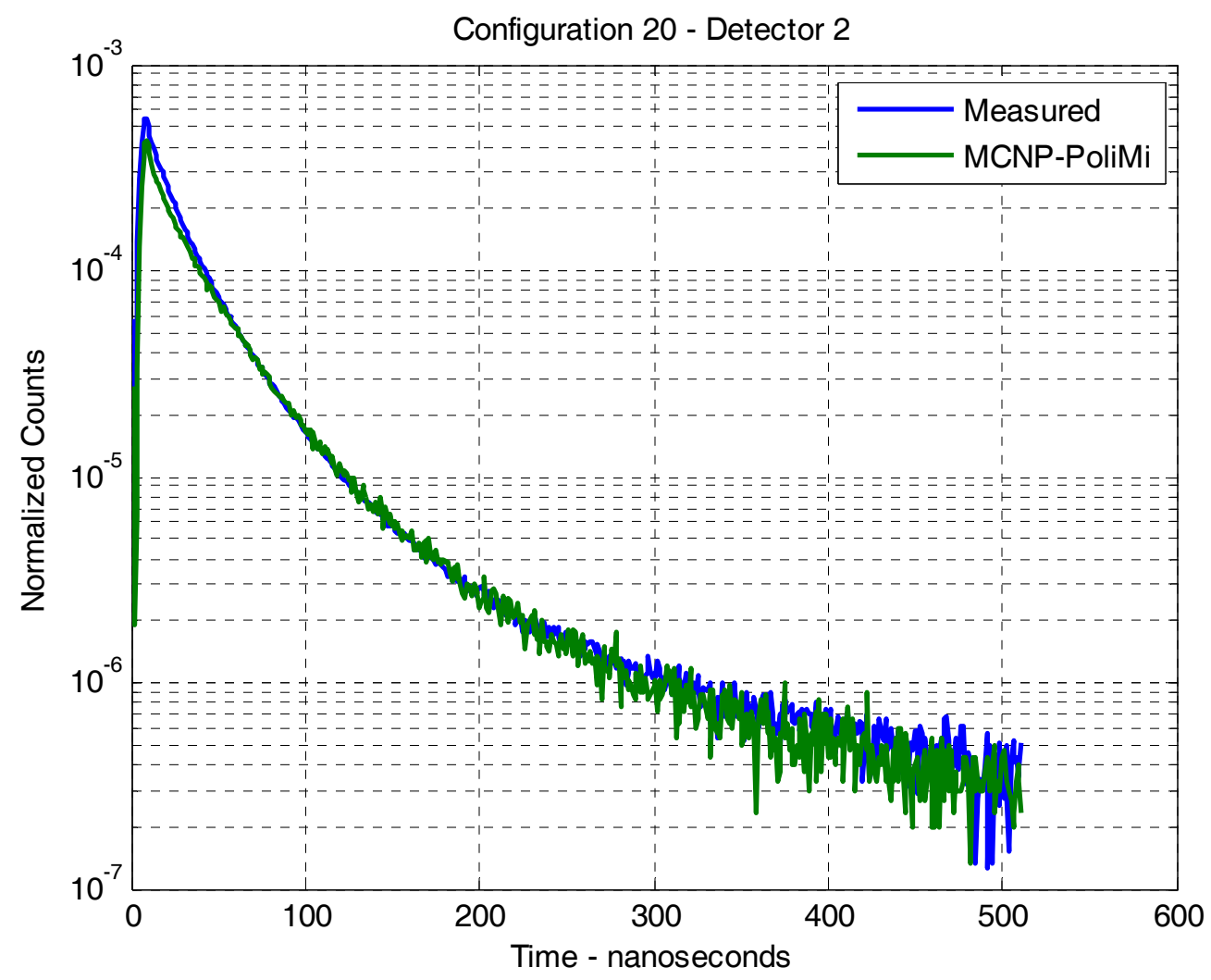

Fig. E.59. Time distribution of counts in detector $\mathbf{2}$ after $\mathbf{C f}$ fission for $\mathbf{5 1 2}$ ns for configuration 20. 


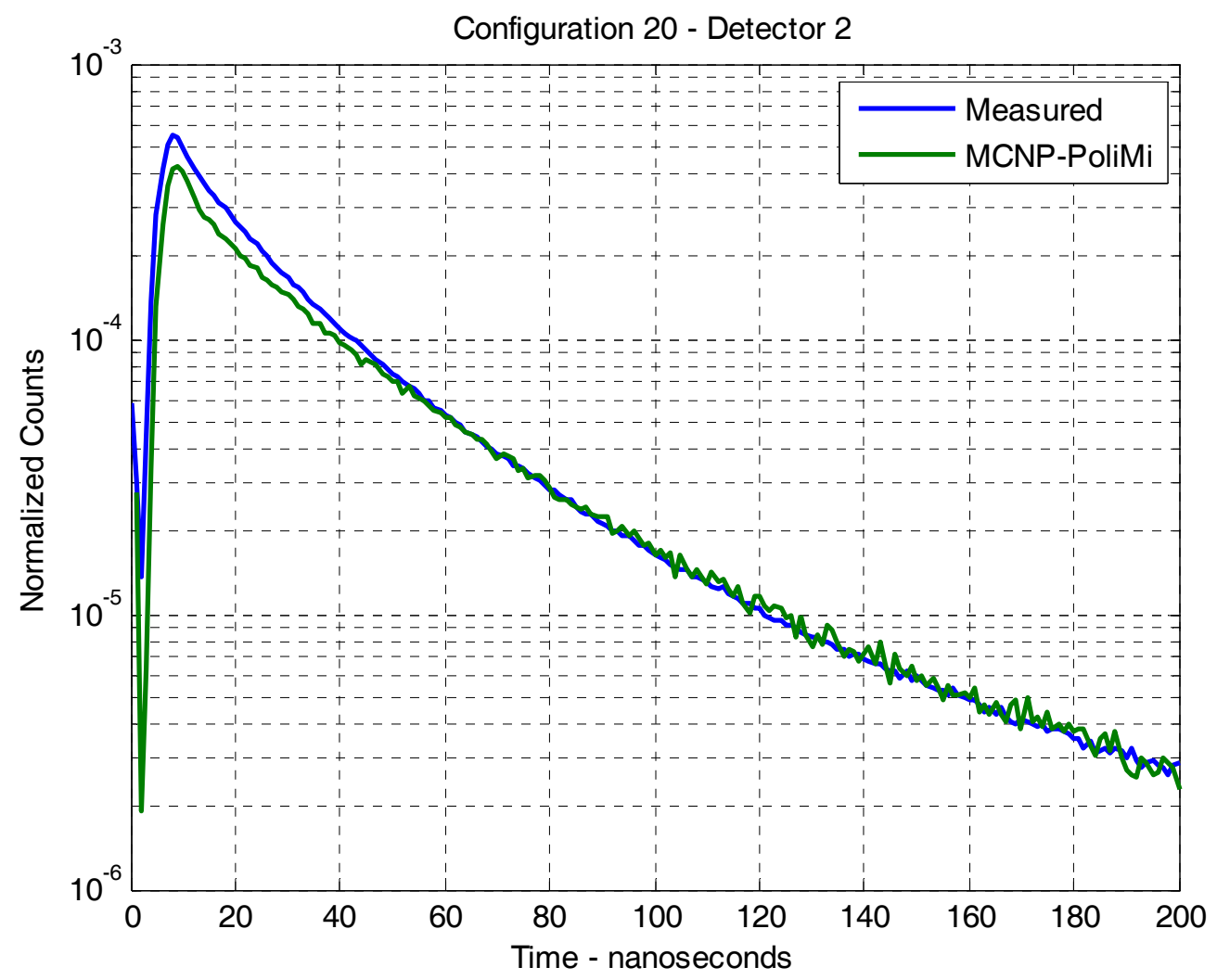

Fig. E.60. Time distribution of counts in detector $\mathbf{2}$ after $\mathbf{C f}$ fission for $200 \mathrm{~ns}$ for configuration 20. 


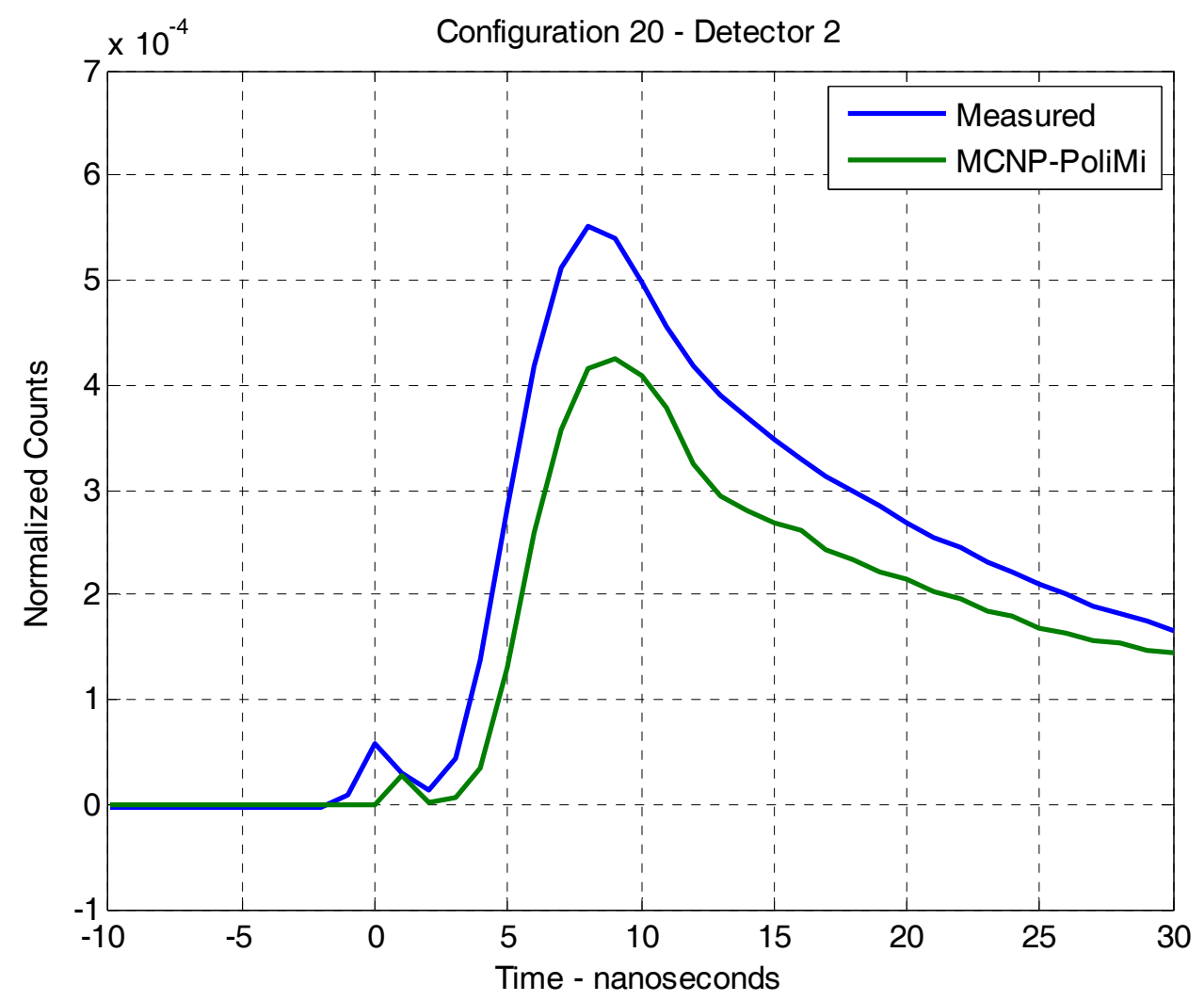

Fig. E.61. Time distribution of counts in detector $\mathbf{2}$ after $\mathbf{C f}$ fission for $\mathbf{5 1 2}$ ns for configuration 30 . 


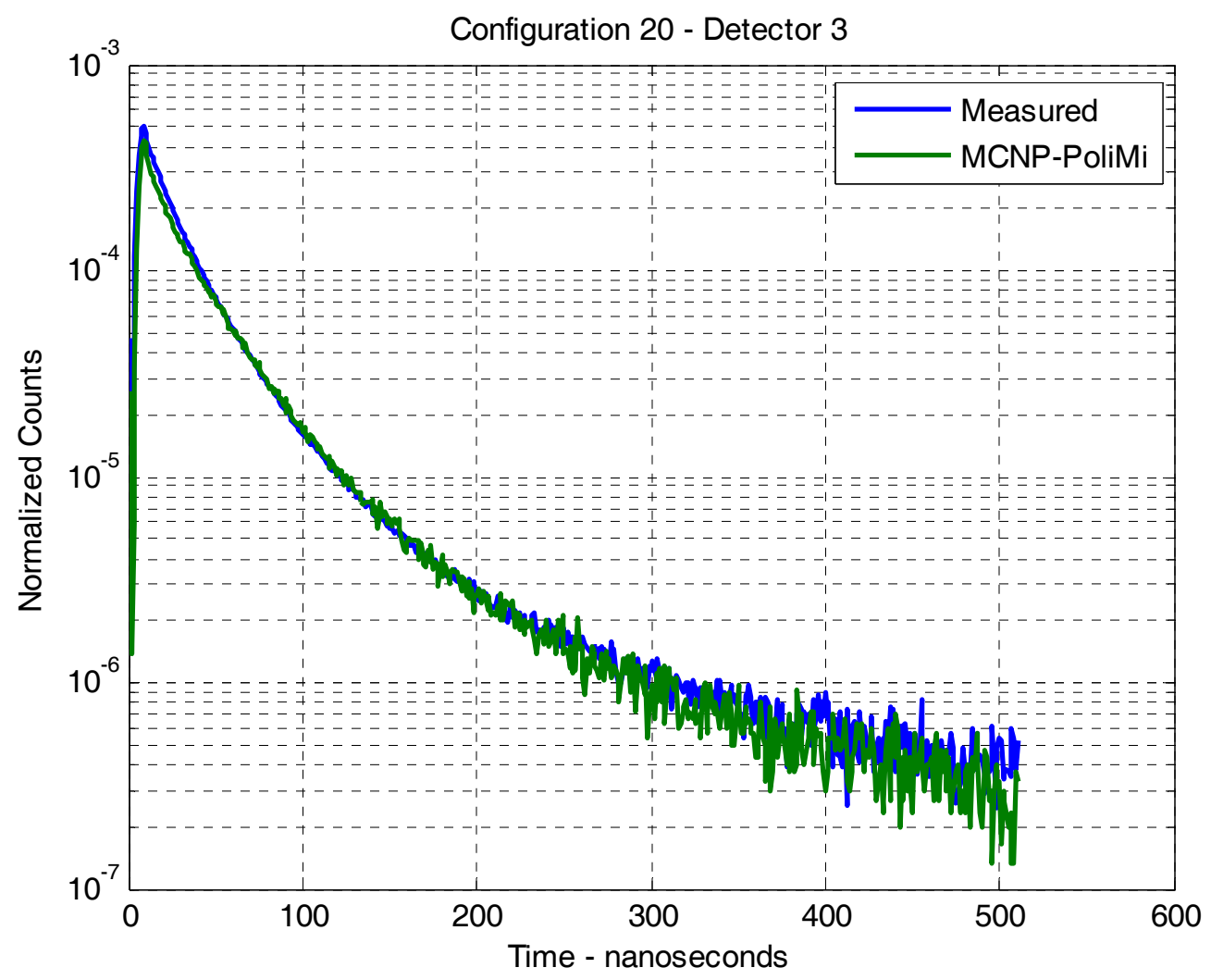

Fig. E.62. Time distribution of counts in detector 3 after $\mathbf{C f}$ fission for $\mathbf{5 1 2}$ ns for configuration 20 . 


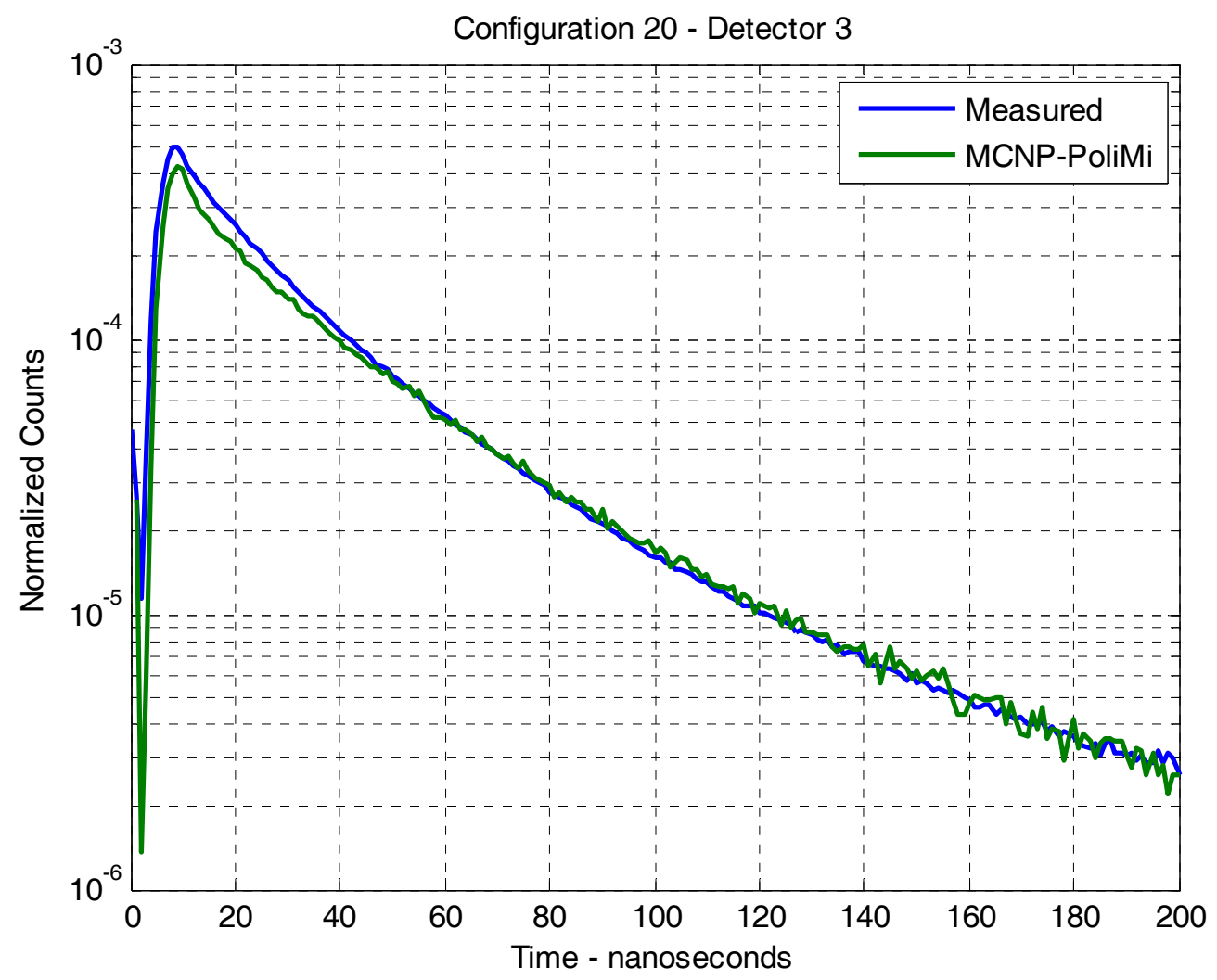

Fig. E.63. Time distribution of counts in detector 3 after $\mathbf{C f}$ fission for $200 \mathrm{~ns}$ for configuration 20. 


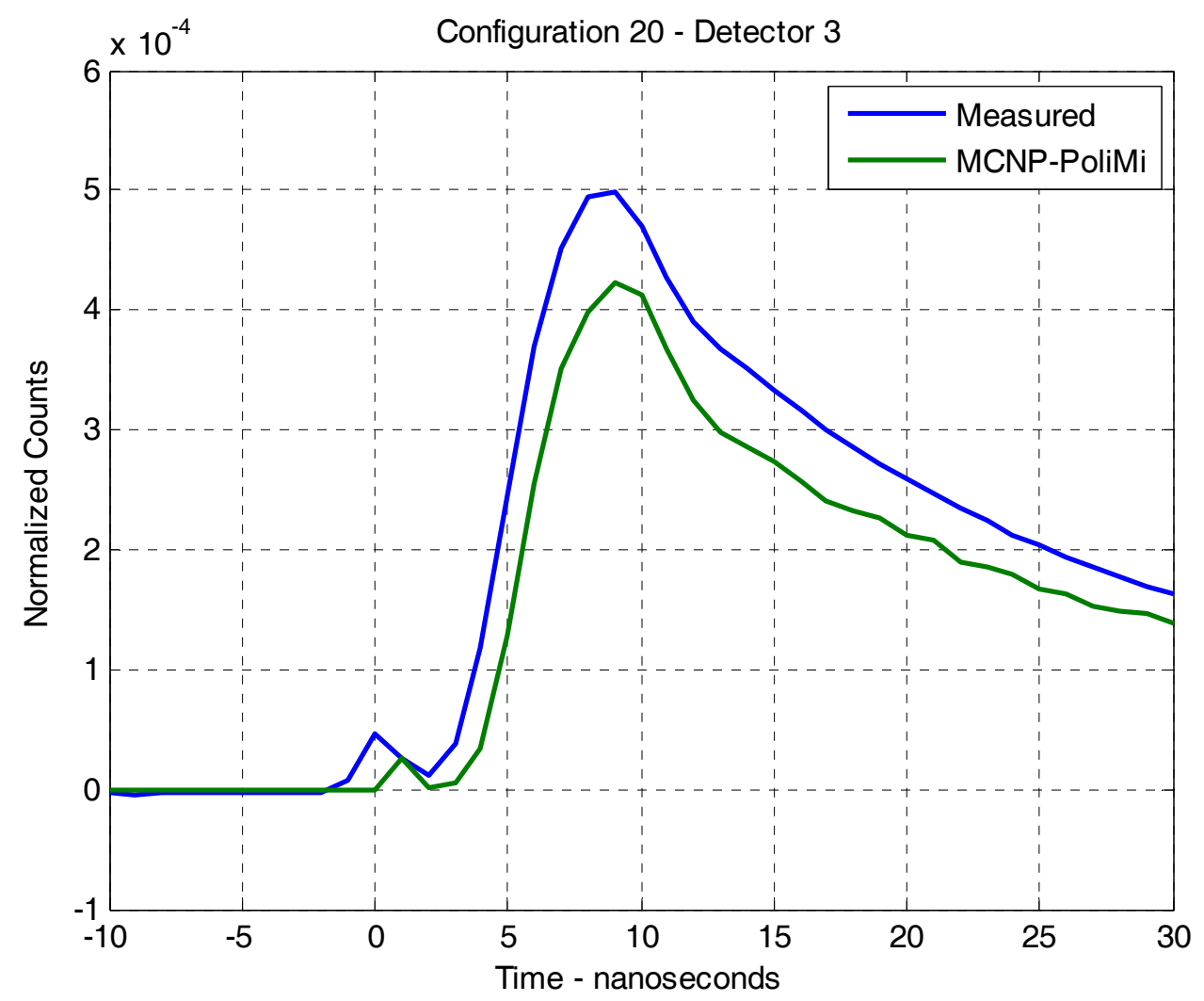

Fig. E.64. Time distribution of counts in detector $\mathbf{3}$ after $\mathbf{C f}$ fission for $\mathbf{3 0}$ ns for configuration 3. 


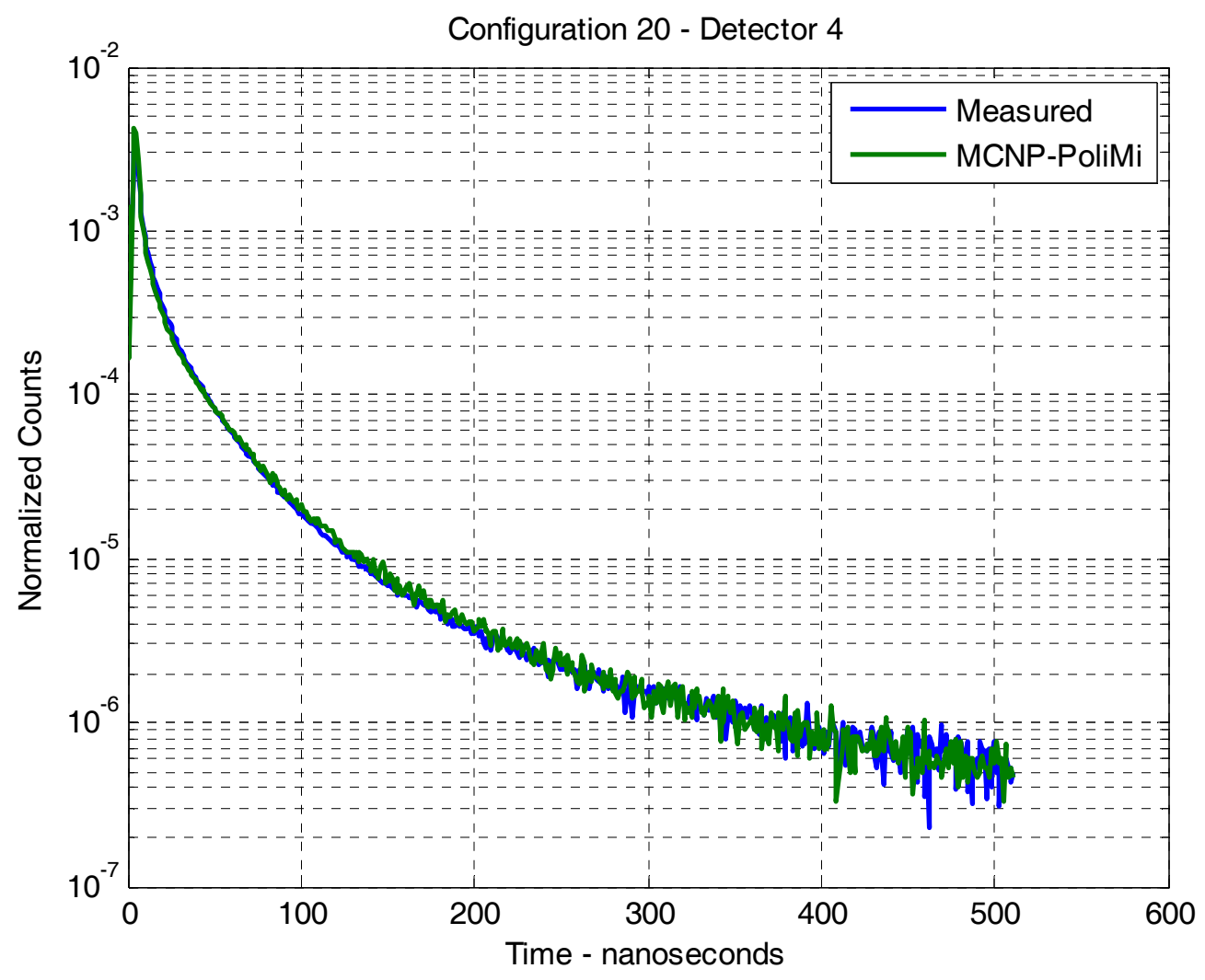

Fig. E.65. Time distribution of counts in detector $\mathbf{4}$ after $\mathbf{C f}$ fission for $\mathbf{5 1 2}$ ns for configuration 20 . 


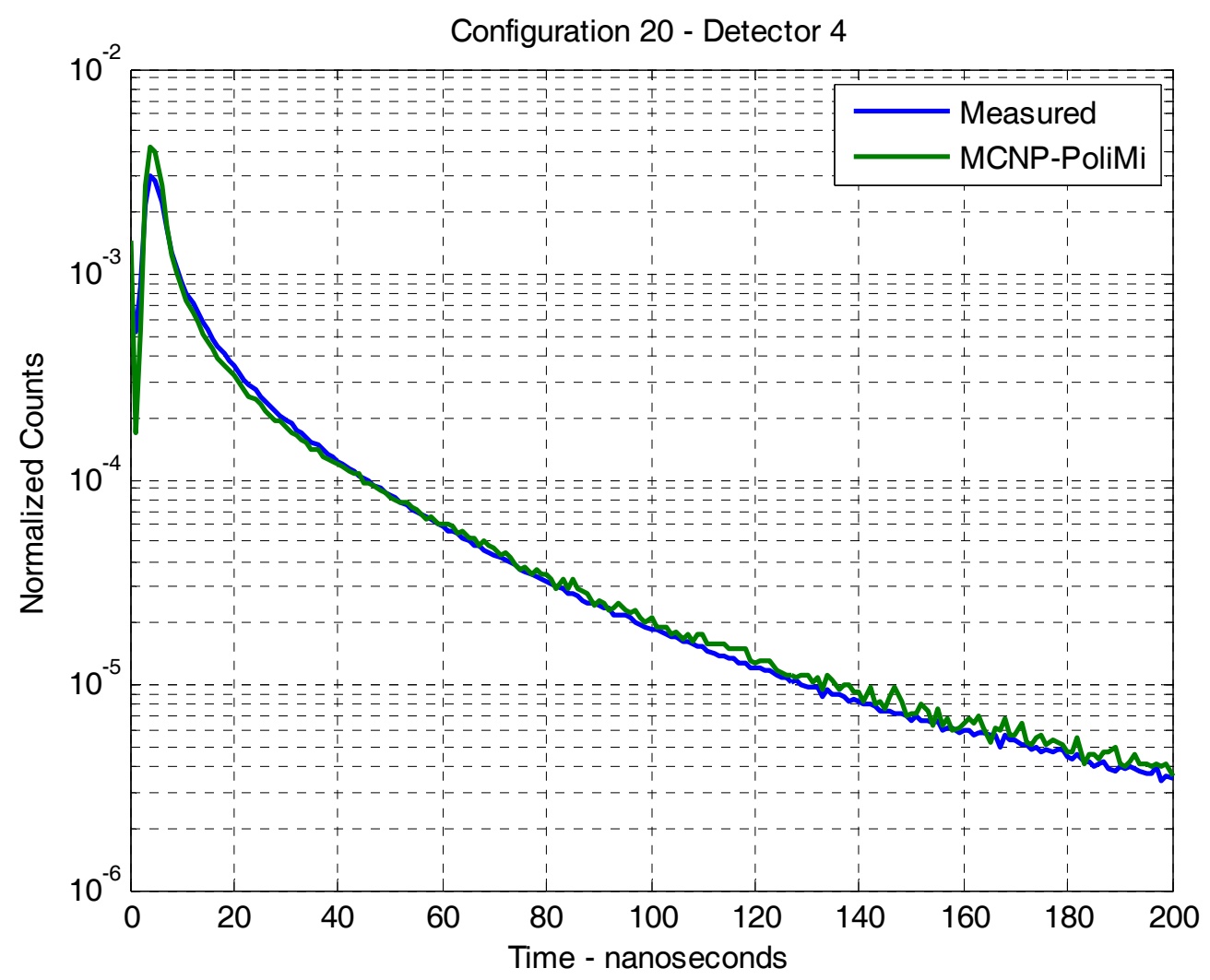

Fig. E.66. Time distribution of counts in detector $\mathbf{4}$ after $\mathbf{C f}$ fission for $200 \mathrm{~ns}$ for configuration 20 . 


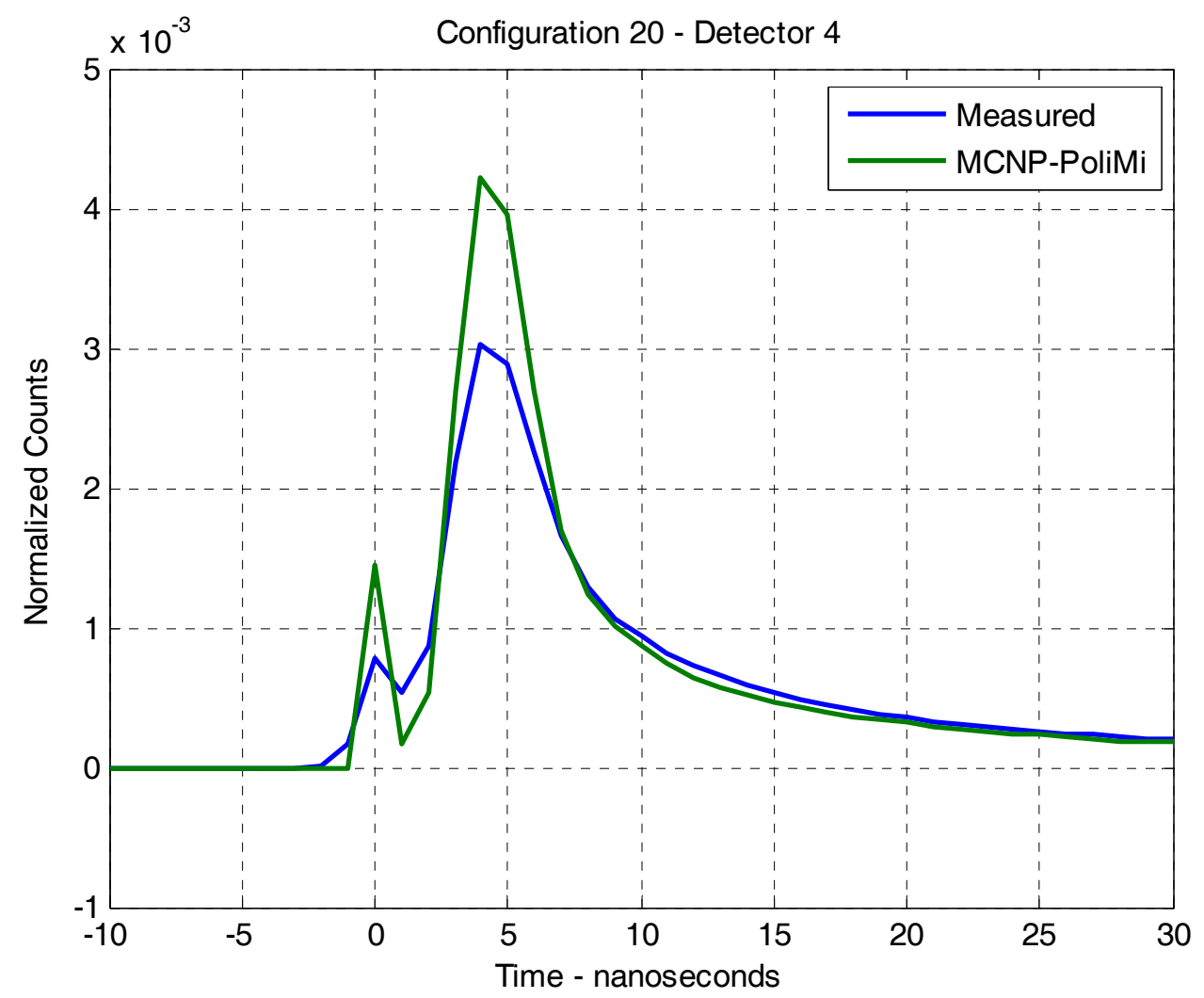

Fig. E.67. Time distribution of counts in detector $\mathbf{4}$ after $\mathbf{C f}$ fission for $\mathbf{3 0}$ ns for configuration 20. 


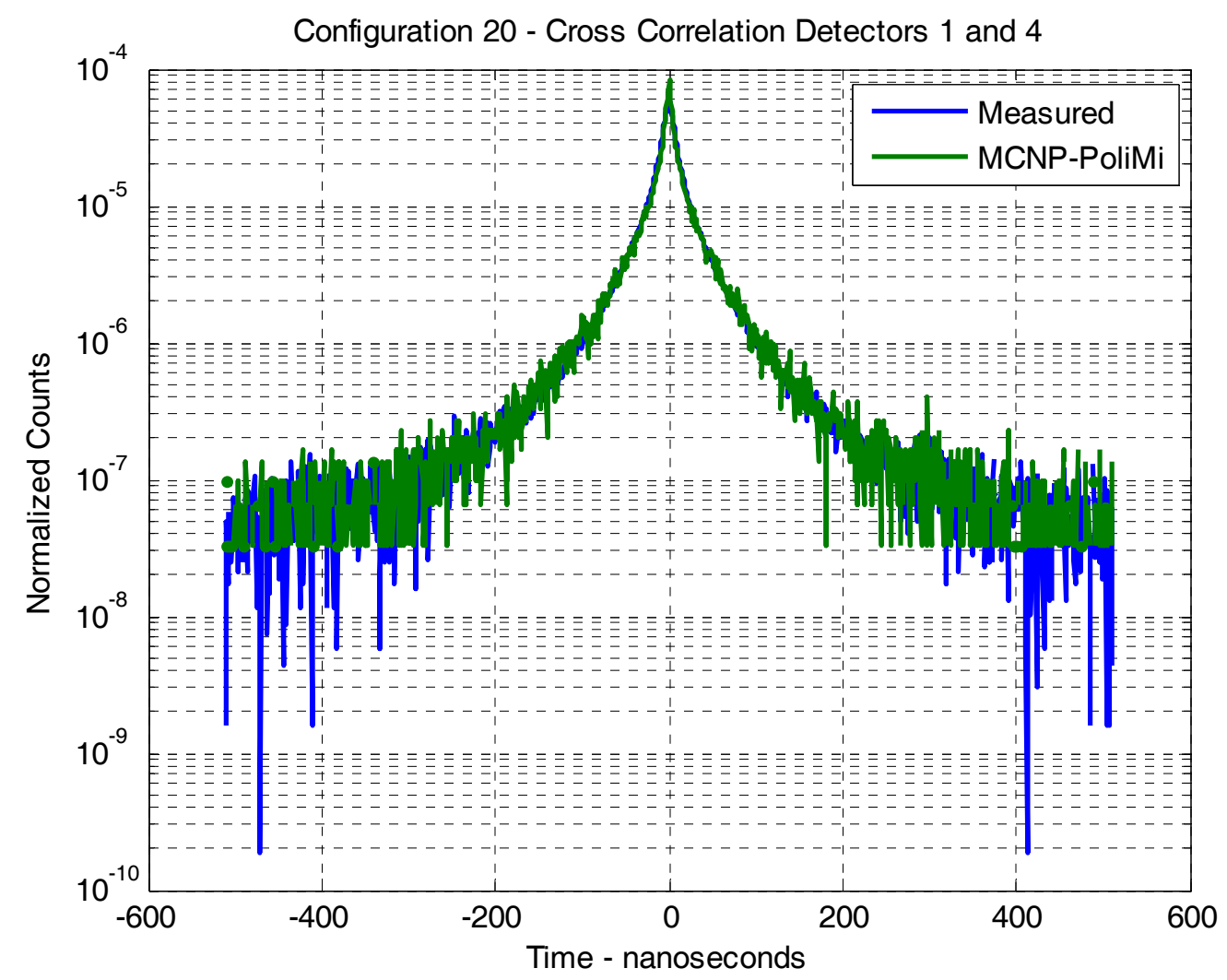

Fig. E.68. Time distribution of counts in detector 4 after a count in detector 1 for 512 ns for configuration 20. 


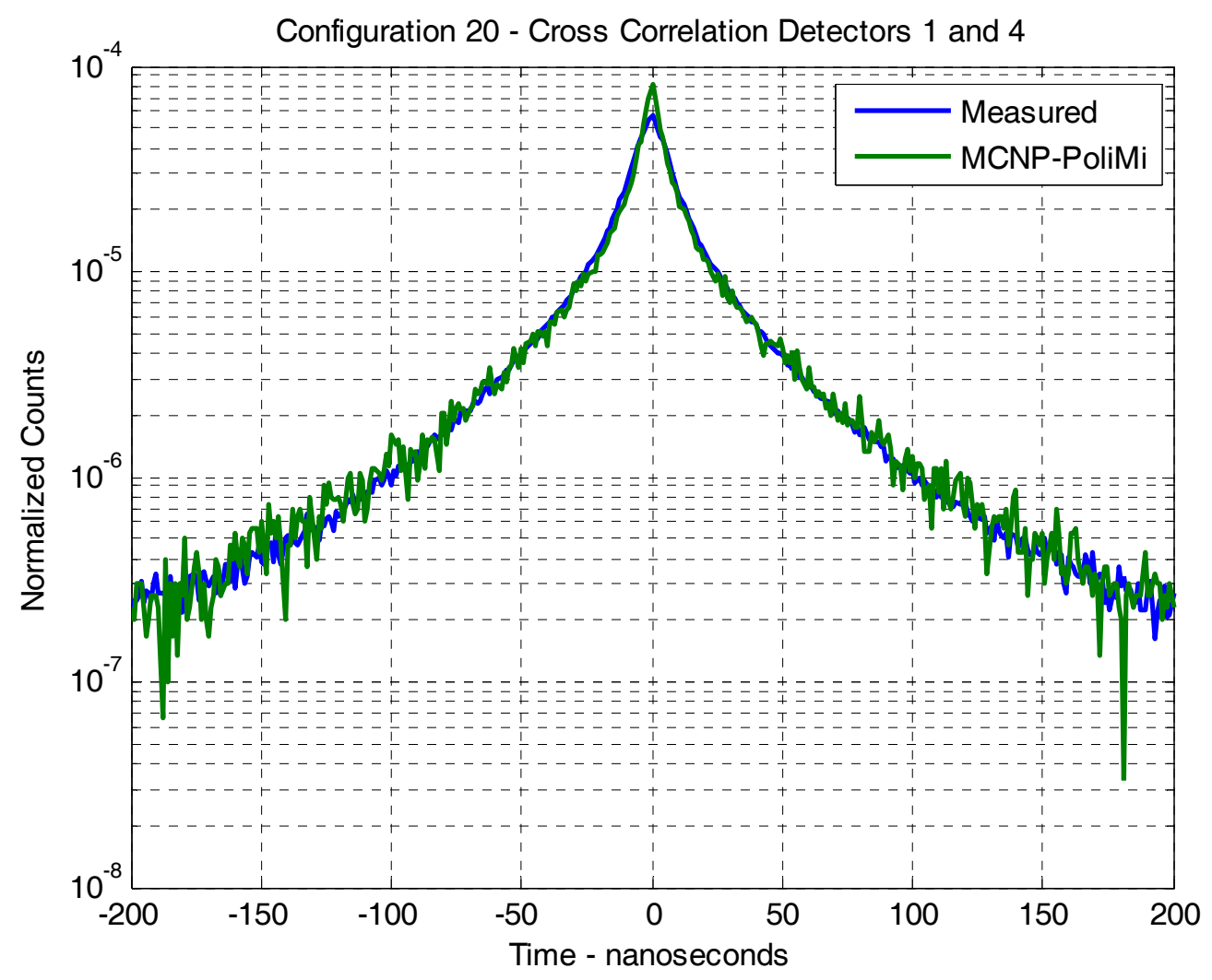

Fig. E.69. Time distribution of counts in detector 4 after a count in detector 1 for 200 ns for configuration 20. 


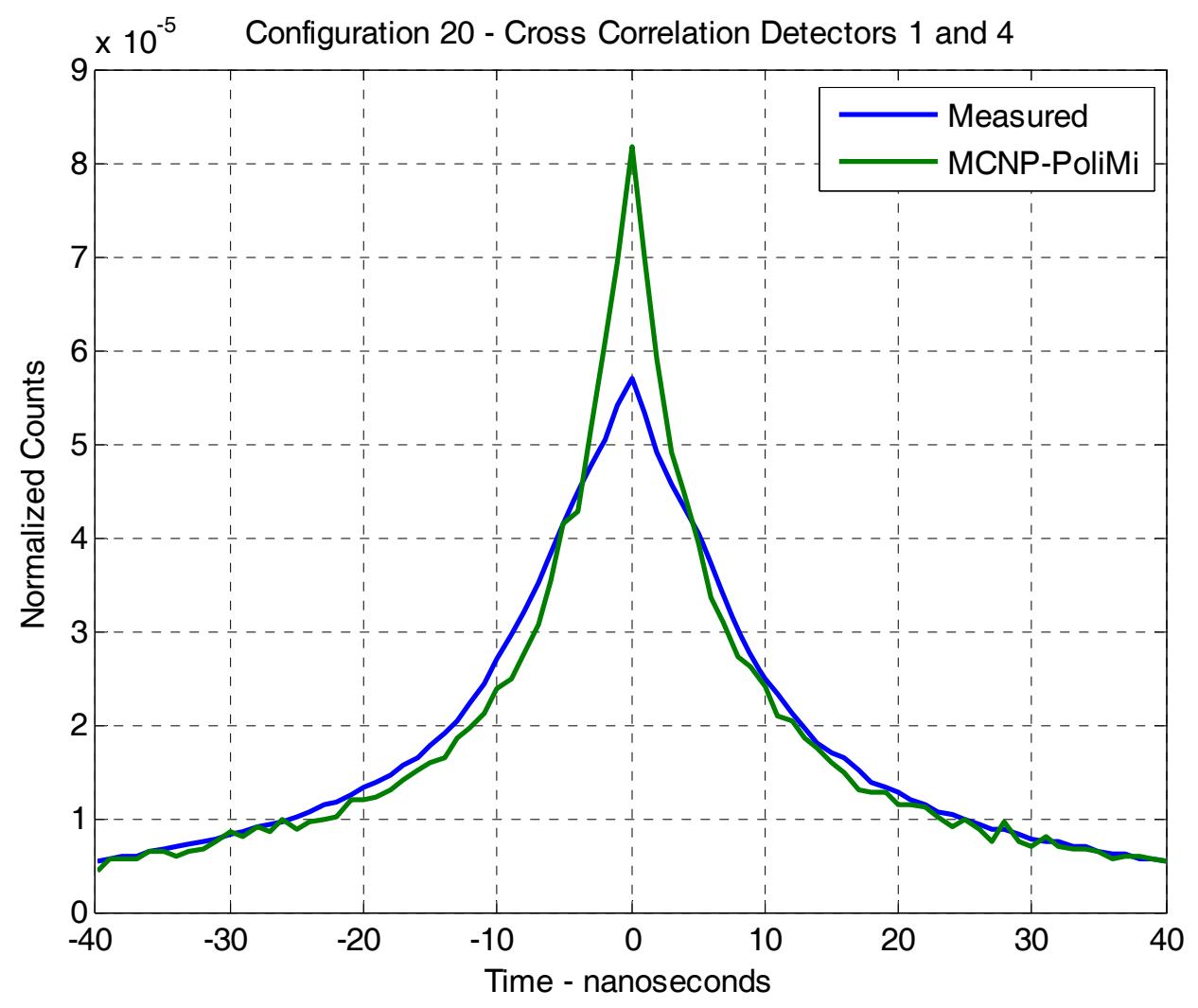

Fig. E.70. Time distribution of counts in detector 4 after a count in detector 1 for $\mathbf{4 0}$ ns for configuration 20. 
Configuration 26:

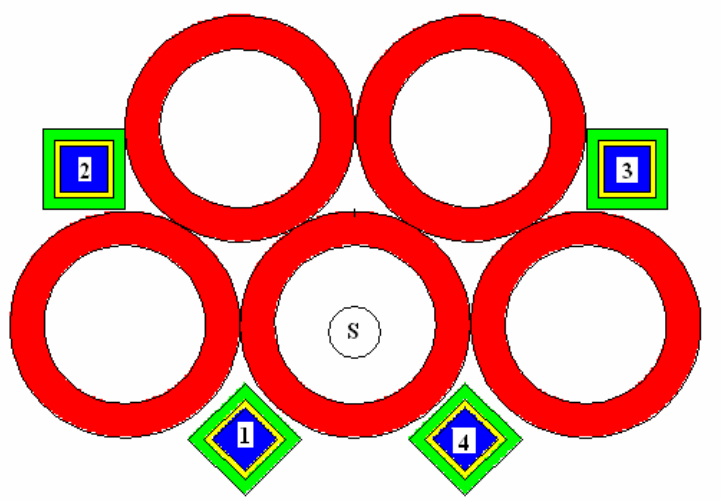

CONFIGURATION 26

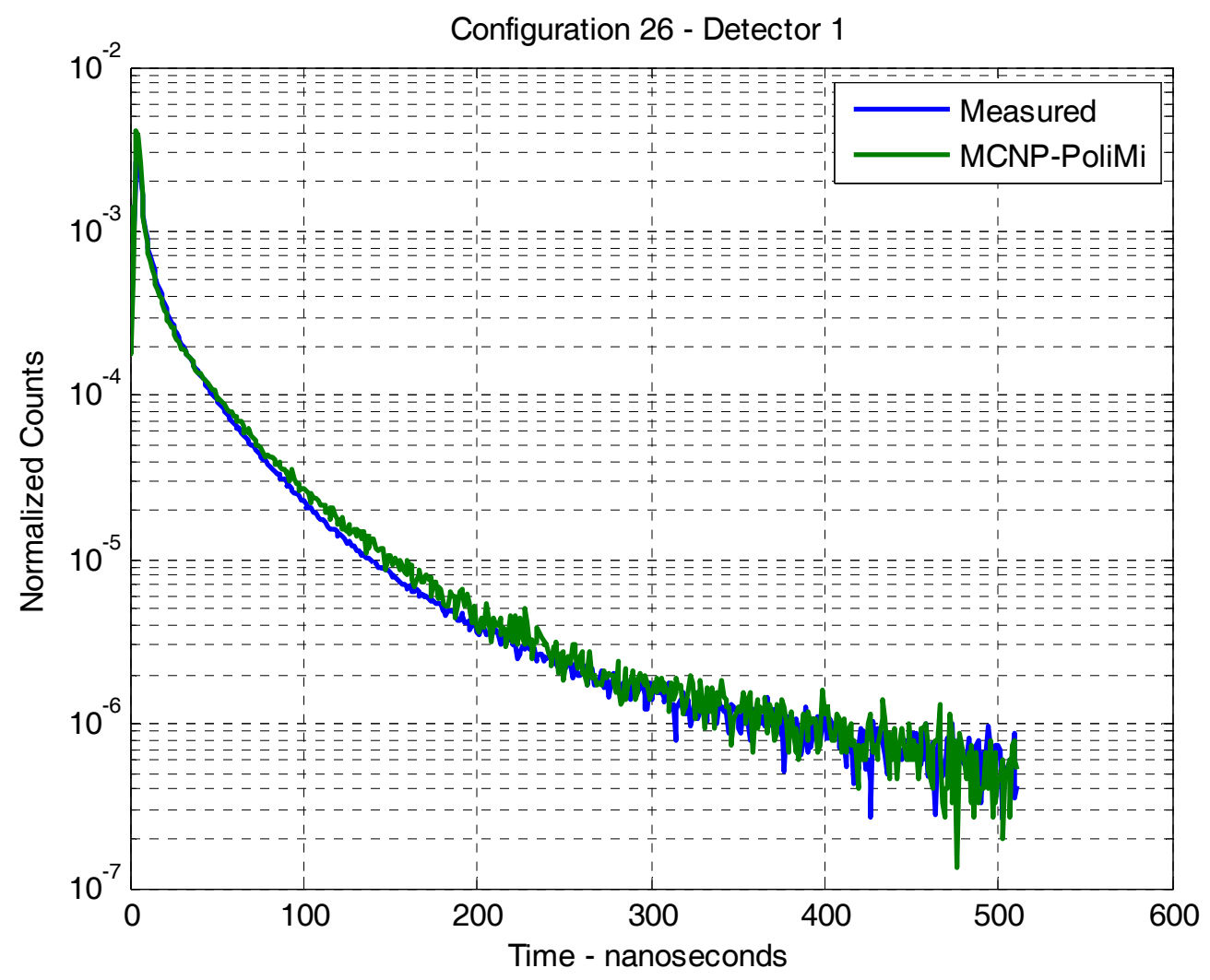

Fig. E.71. Time distribution of counts in detector 1 after $\mathbf{C f}$ fission for $\mathbf{5 1 2}$ ns for configuration 26. 


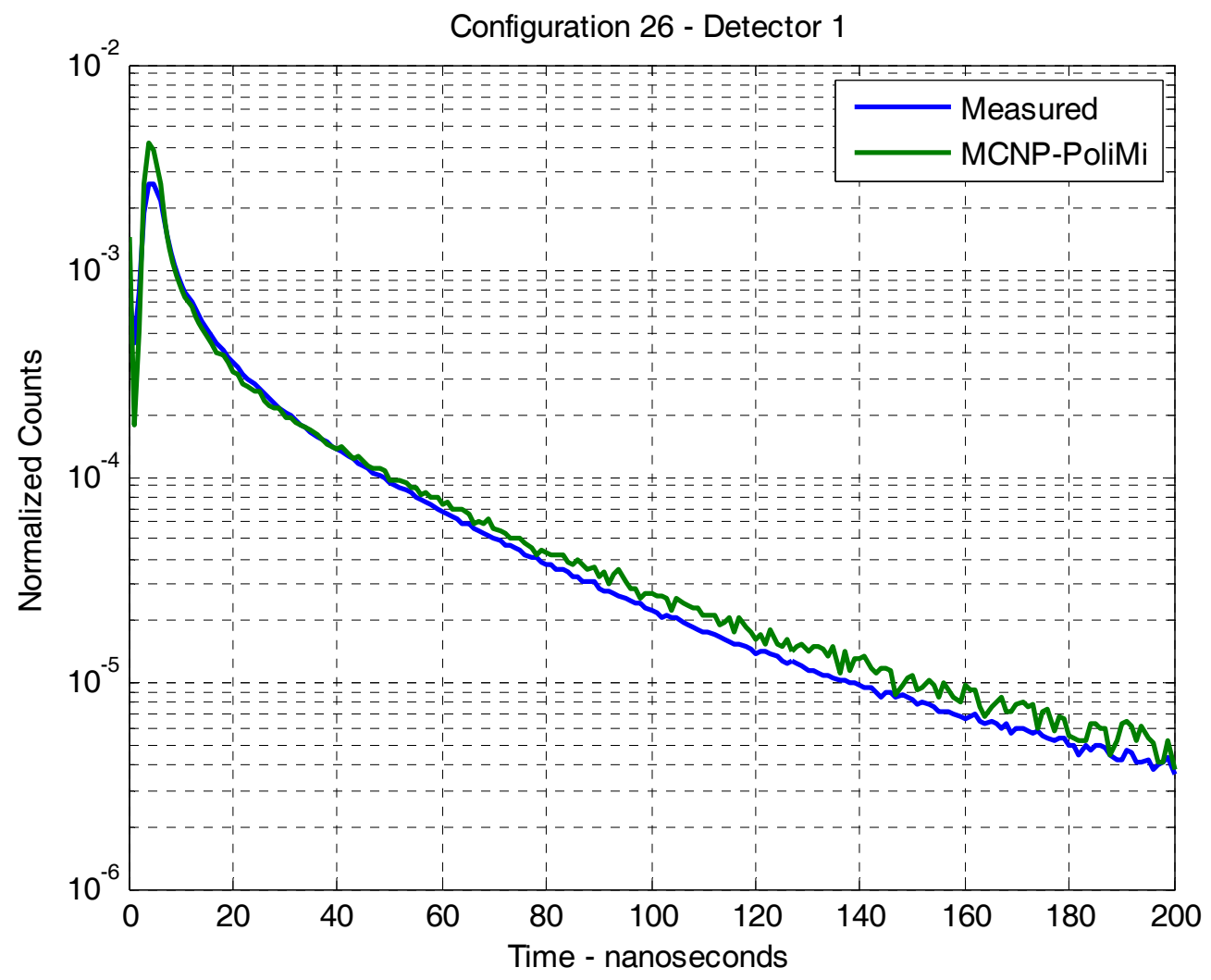

Fig. E.72. Time distribution of counts in detector $\mathbf{1}$ after $\mathbf{C f}$ fission for $200 \mathrm{~ns}$ for configuration 26. 


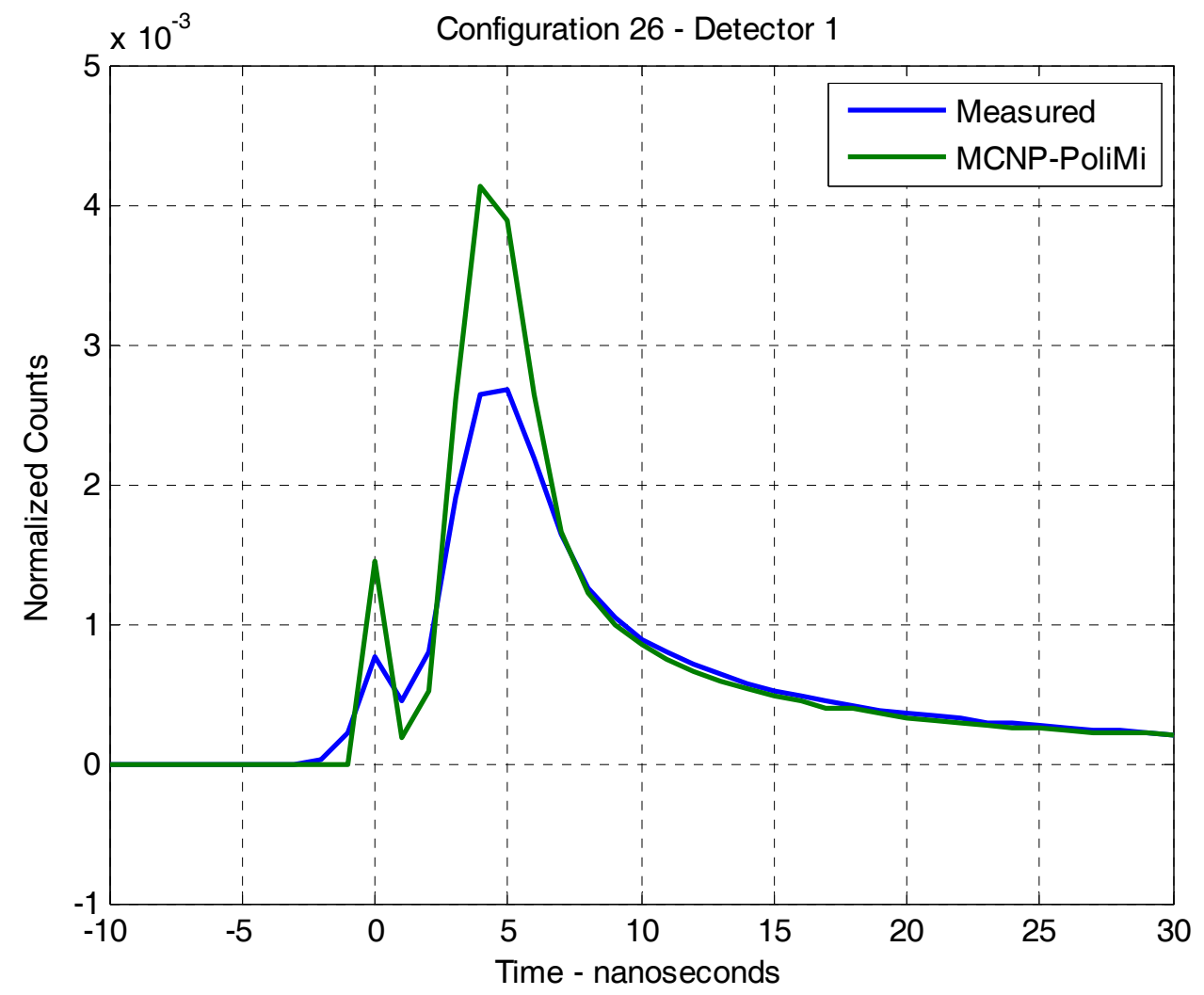

Fig. E.73. Time distribution of counts in detector $\mathbf{1}$ after $\mathbf{C f}$ fission for $\mathbf{3 0}$ ns for configuration 26. 


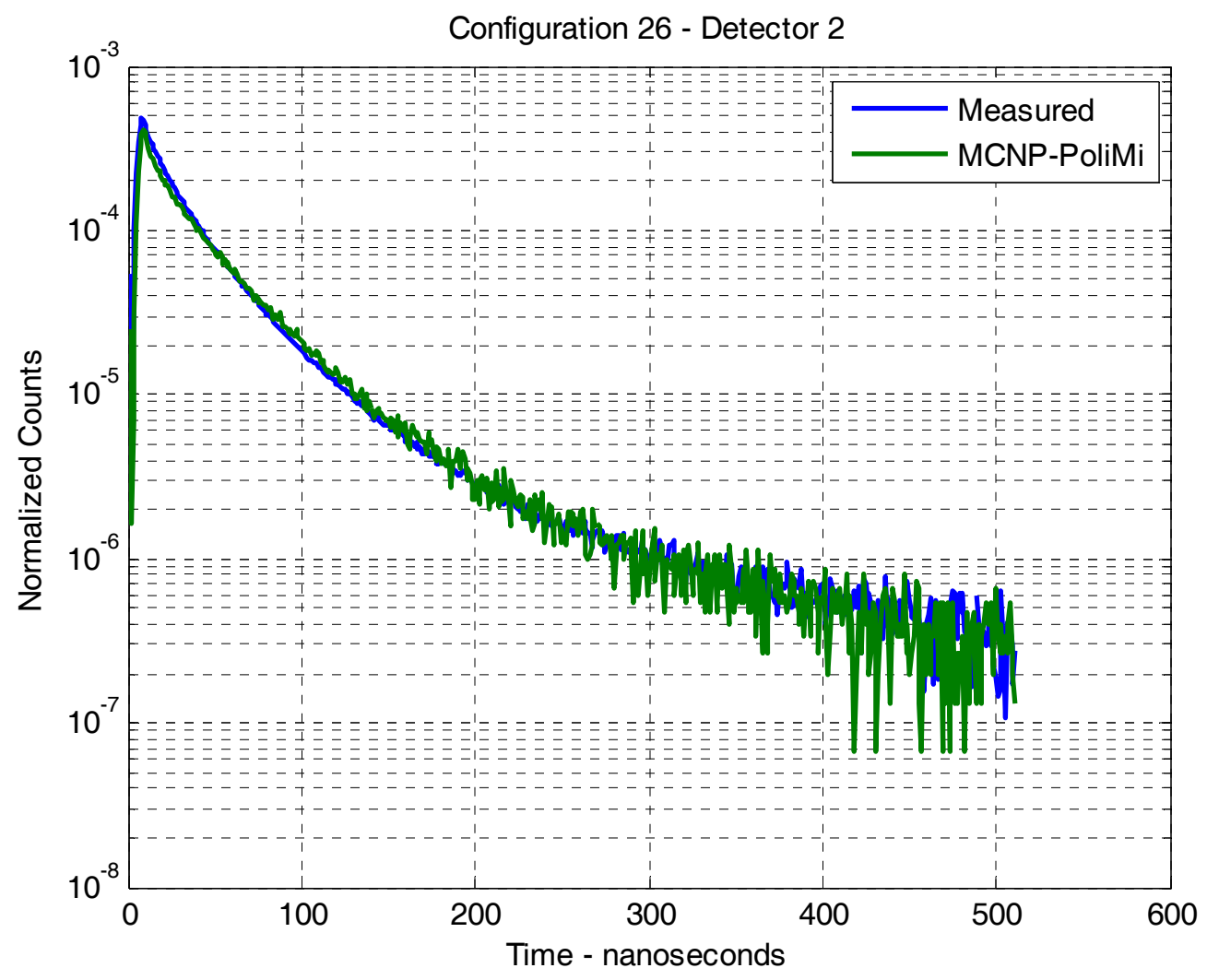

Fig. E.74. Time distribution of counts in detector $\mathbf{2}$ after $\mathbf{C f}$ fission for $\mathbf{5 1 2}$ ns for configuration 26. 


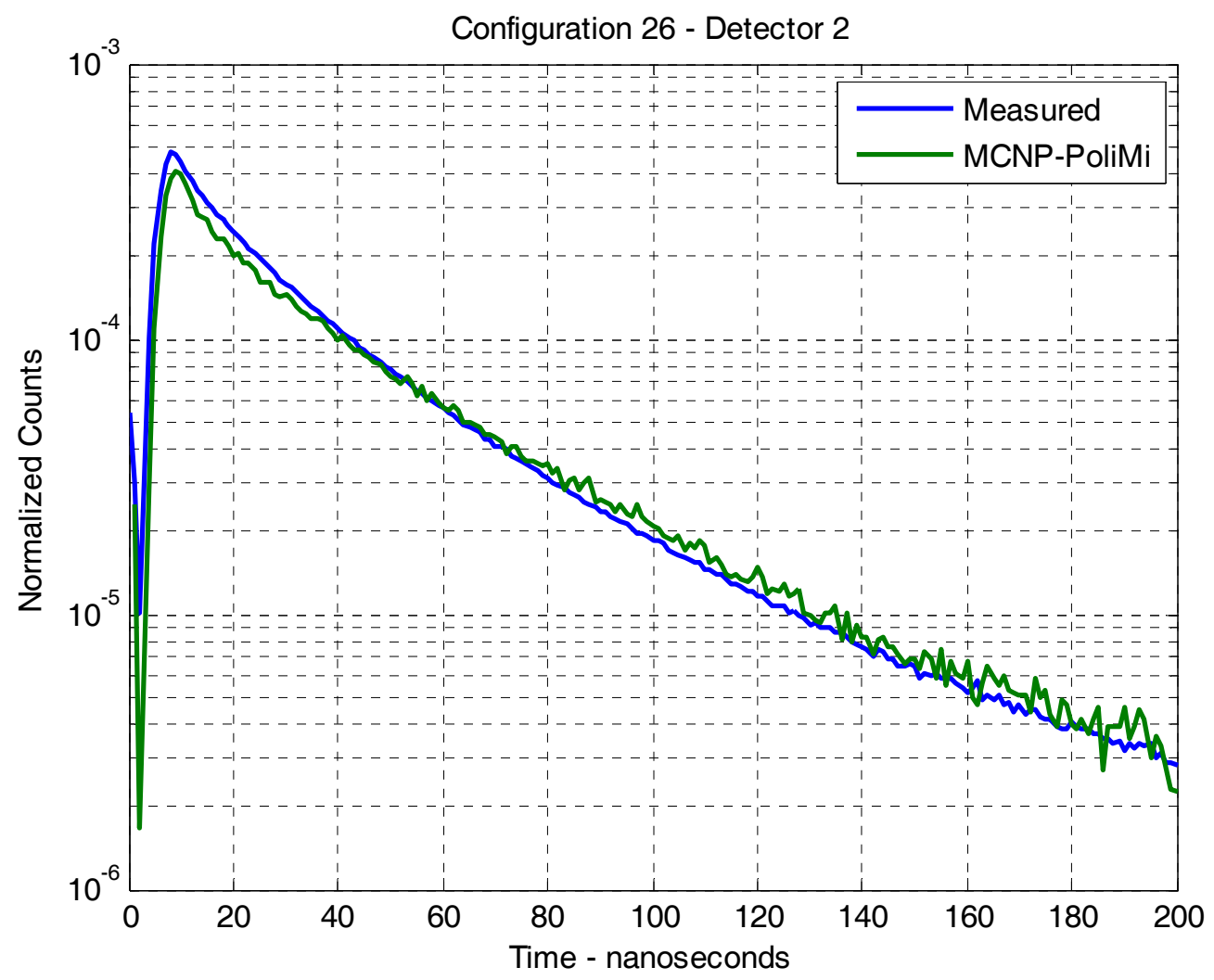

Fig. E.75. Time distribution of counts in detector 2 after $\mathbf{C f}$ fission for $200 \mathrm{~ns}$ for configuration 26. 


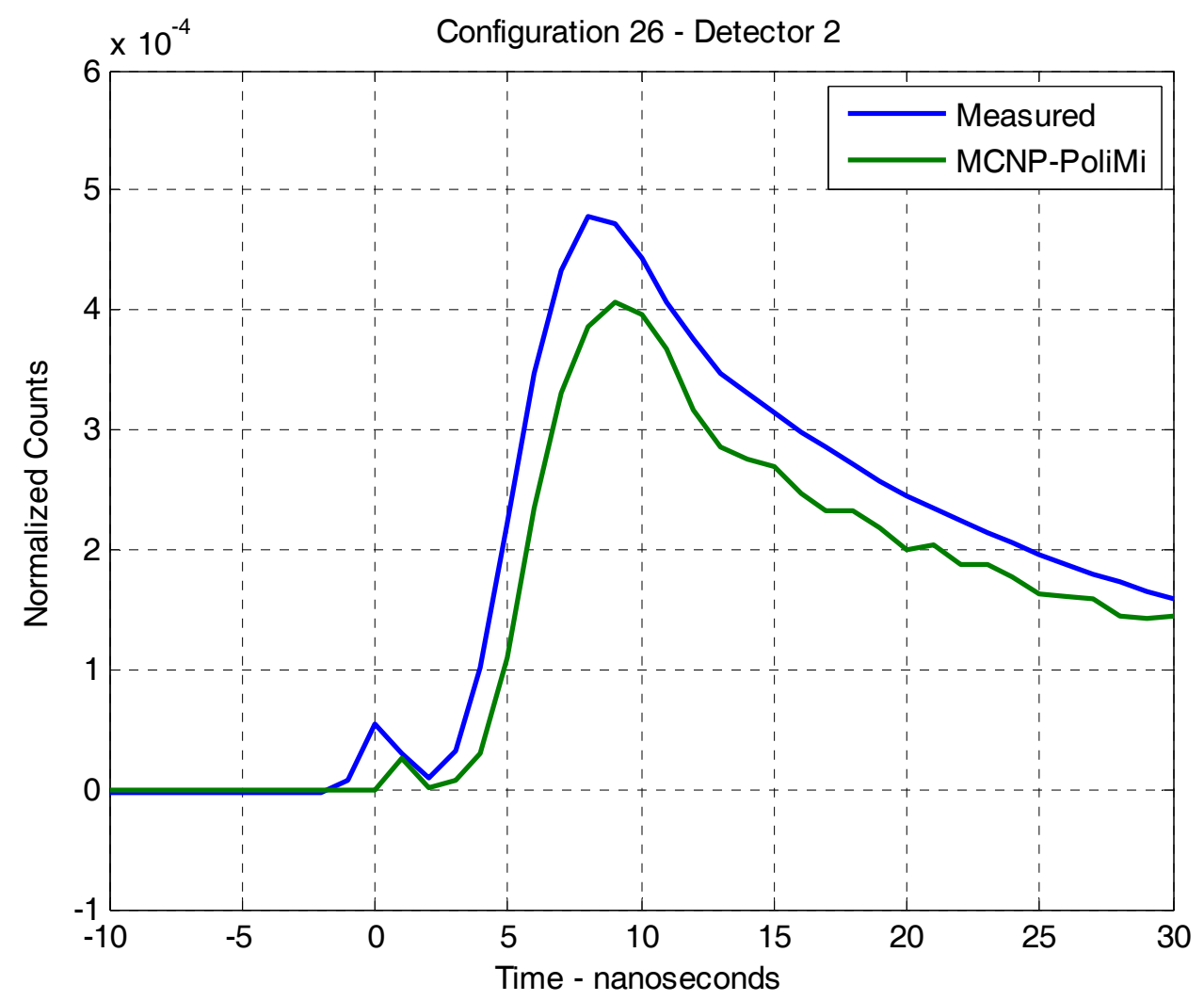

Fig. E.76. Time distribution of counts in detector 2 after $\mathbf{C f}$ fission for $30 \mathrm{~ns}$ for configuration 26. 


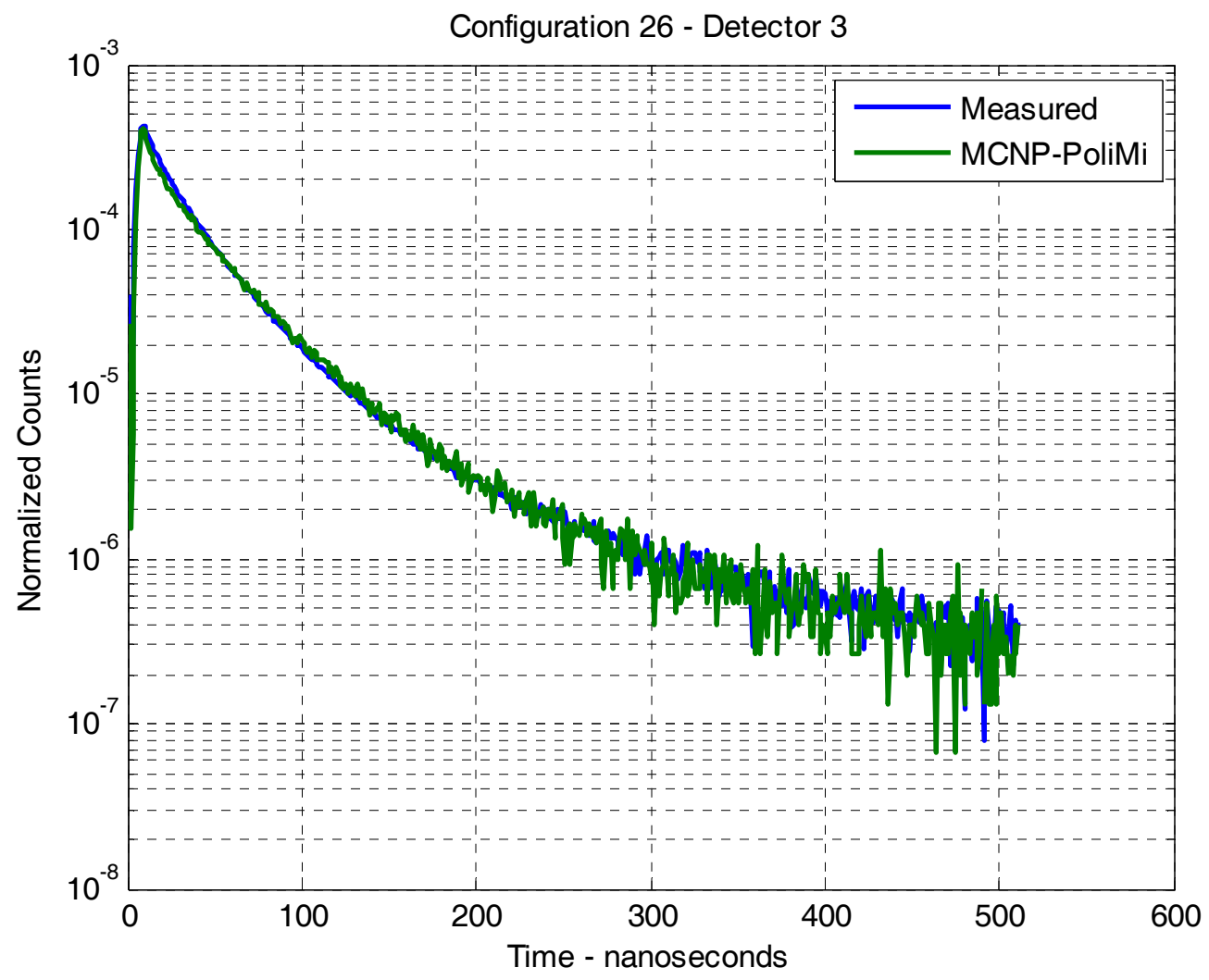

Fig. E.77. Time distribution of counts in detector 3 after $\mathbf{C f}$ fission for $\mathbf{5 1 2}$ ns for configuration 26. 


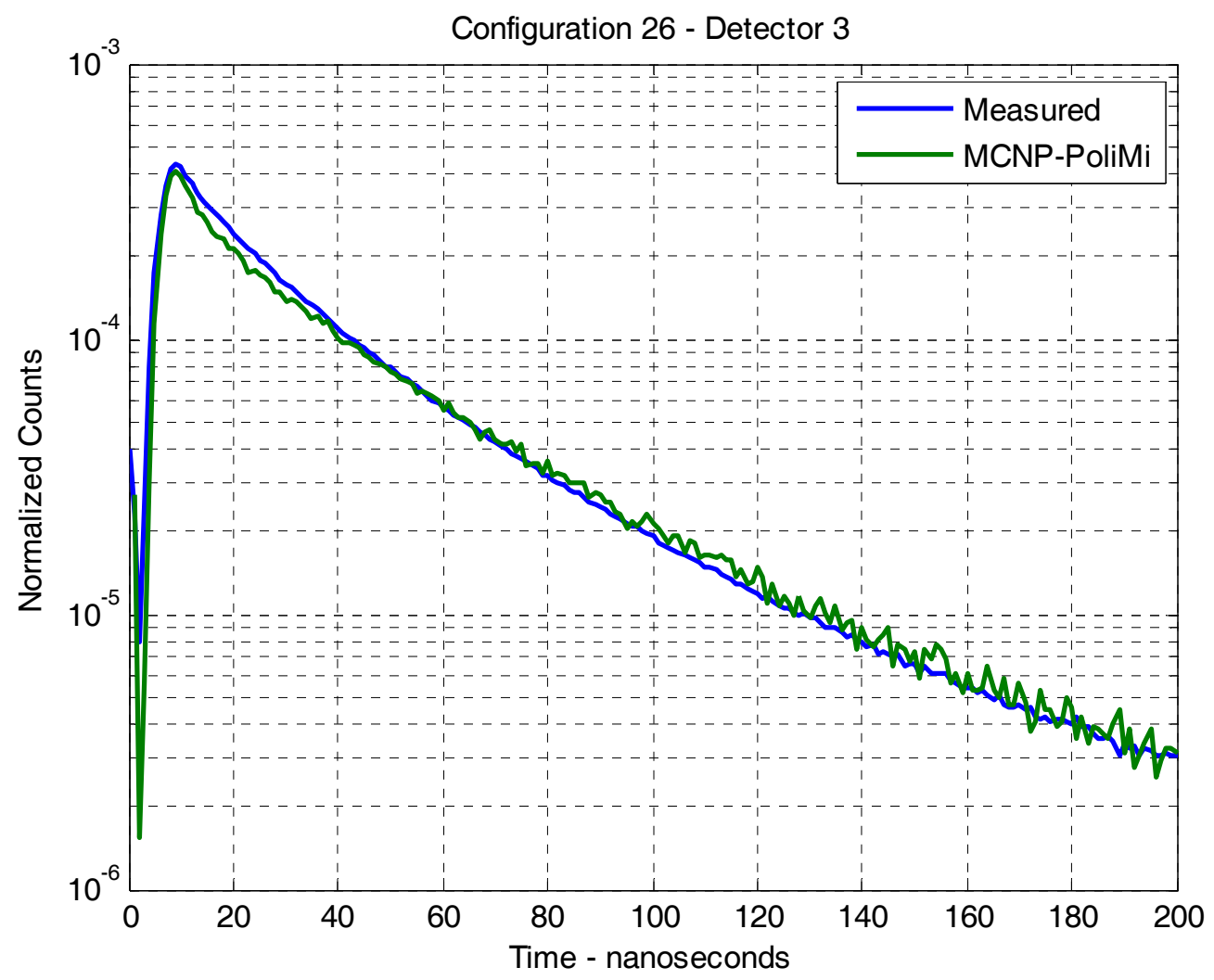

Fig. E.78. Time distribution of counts in detector 3 after $\mathbf{C f}$ fission for $200 \mathrm{~ns}$ for configuration 26. 


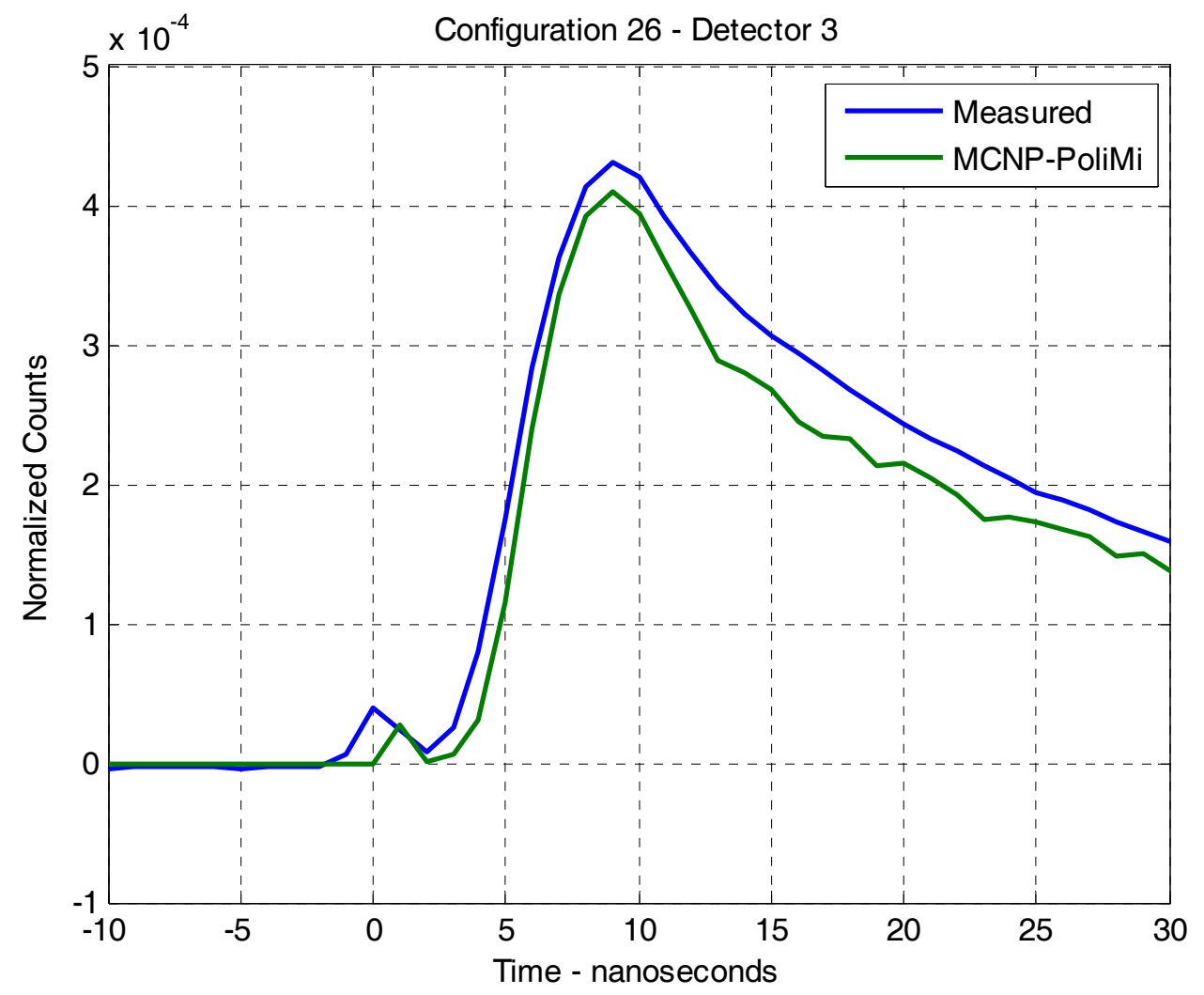

Fig. E.79. Time distribution of counts in detector $\mathbf{3}$ after $\mathbf{C f}$ fission for $\mathbf{3 0}$ ns for configuration 26. 


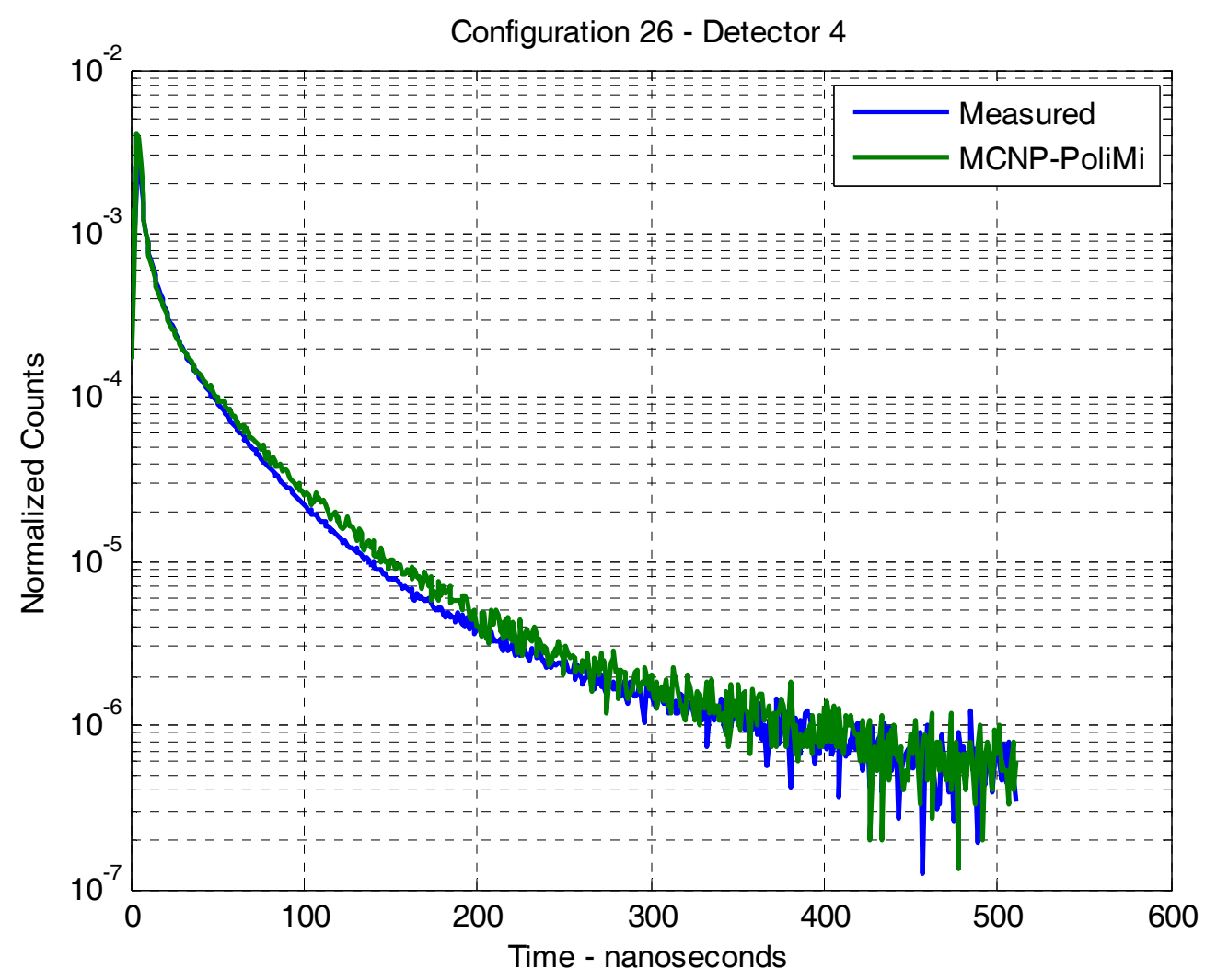

Fig. E.80. Time distribution of counts in detector $\mathbf{4}$ after $\mathbf{C f}$ fission for $\mathbf{5 1 2}$ ns for configuration 26 . 


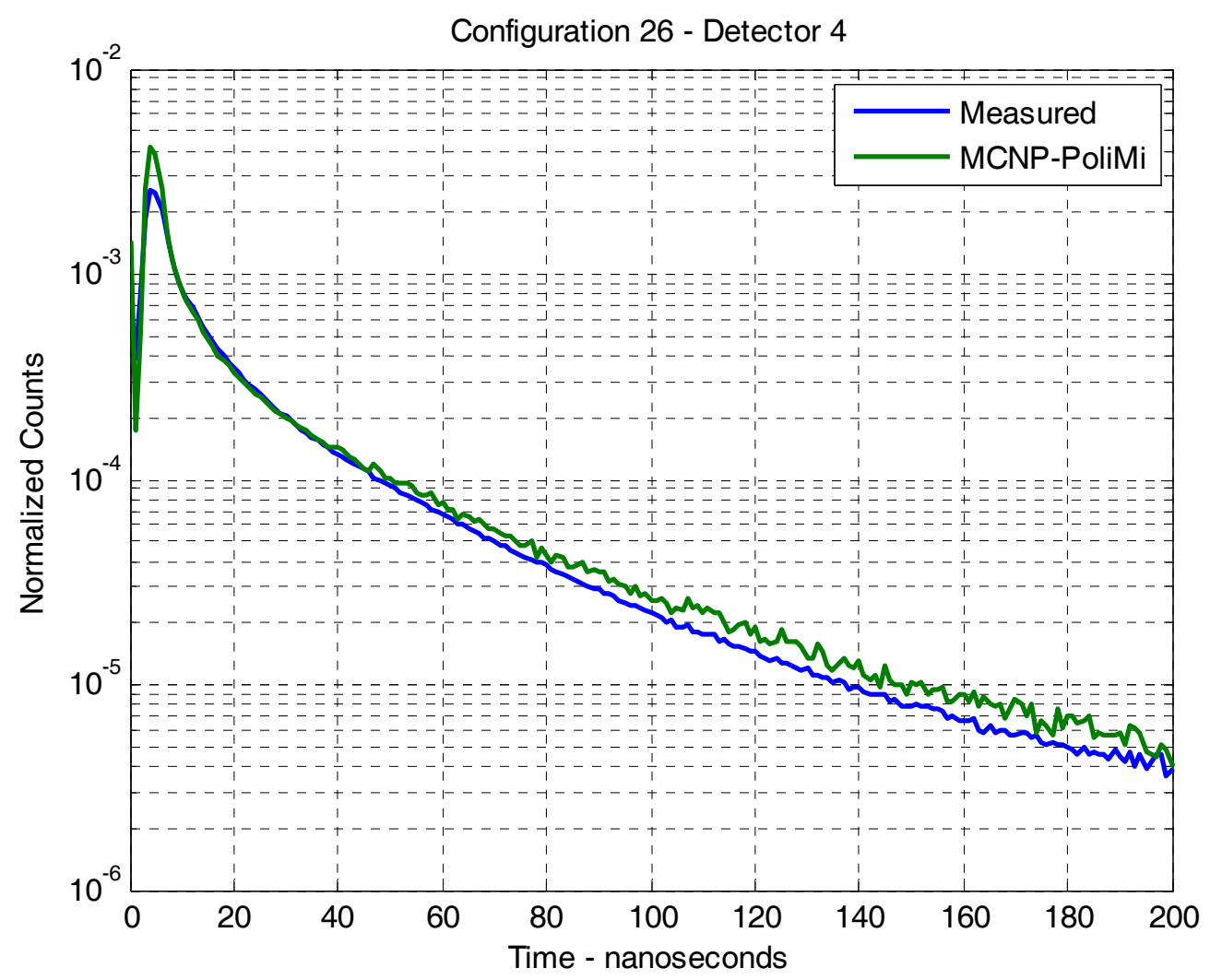

Fig. E.81. Time distribution of counts in detector $\mathbf{4}$ after $\mathbf{C f}$ fission for $200 \mathrm{~ns}$ for configuration 26. 


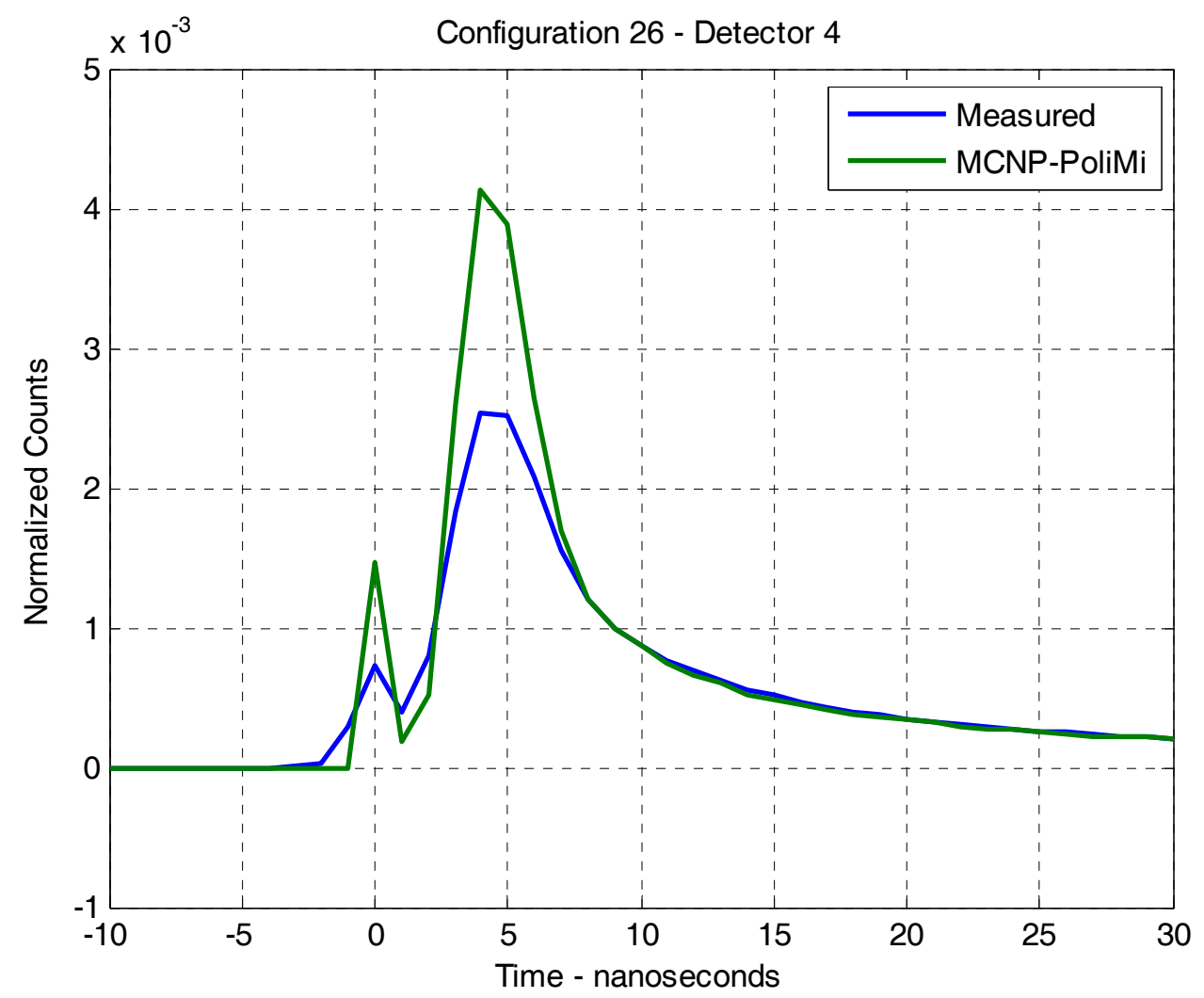

Fig. E.82. Time distribution of counts in detector $\mathbf{4}$ after $\mathbf{C f}$ fission for $\mathbf{3 0} \mathbf{n s}$ for configuration 26. 


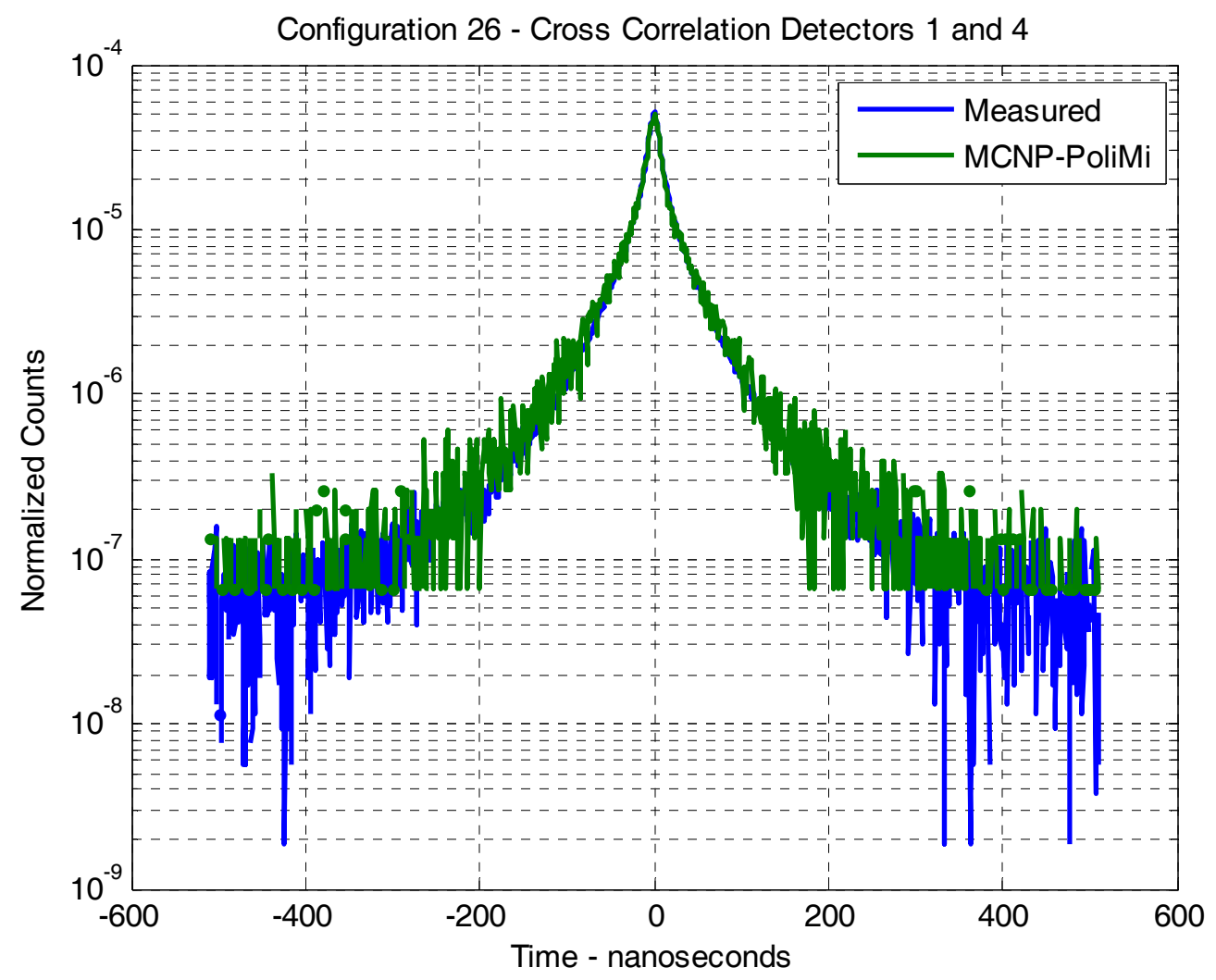

Fig. E.83. Time distribution of counts in detector 4 after a count in detector 1 for 512 ns for configuration 26. 


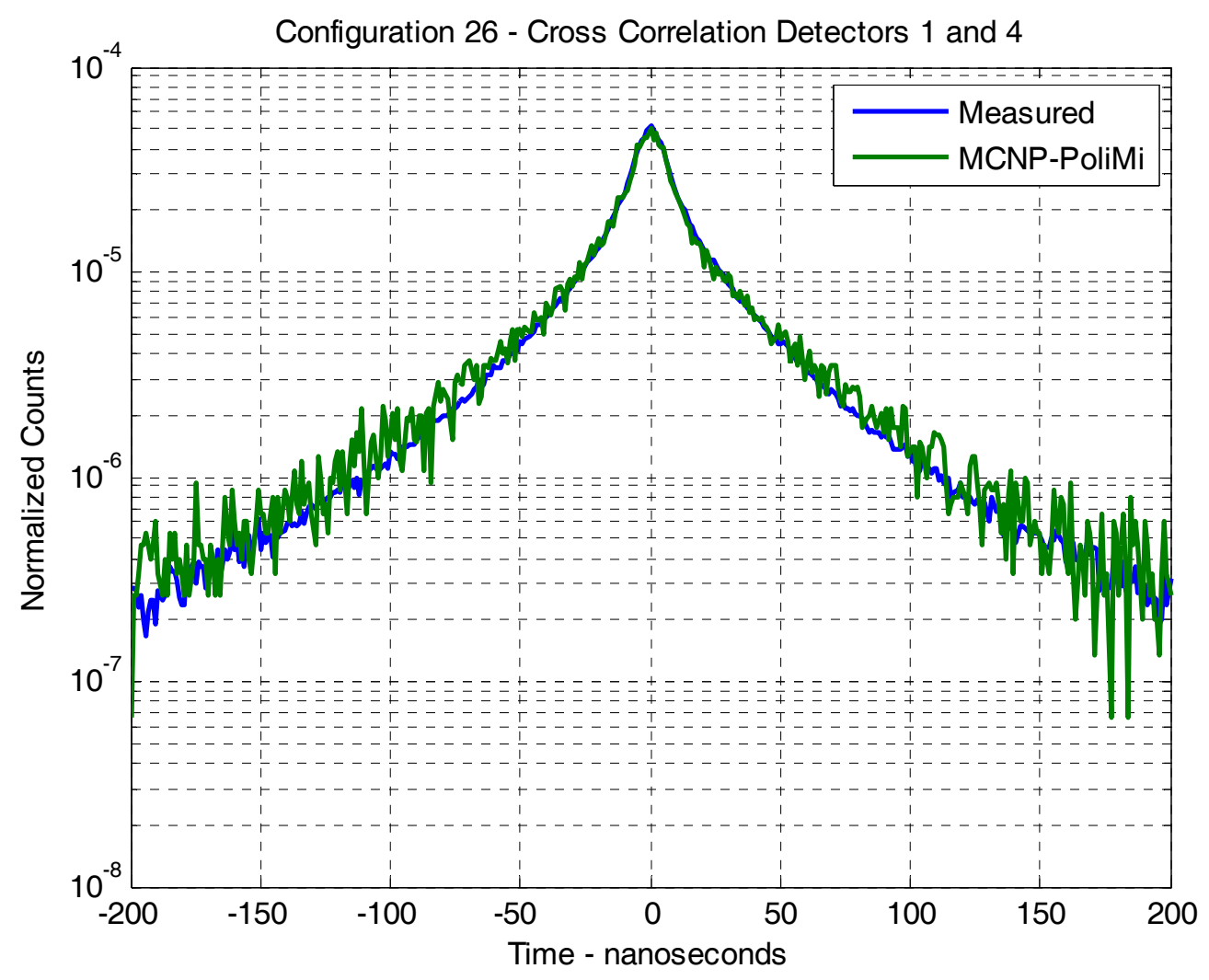

Fig. E.84. Time distribution of counts in detector 1 and 4 after $\mathbf{C f}$ fission for 512 ns for configuration 26. 


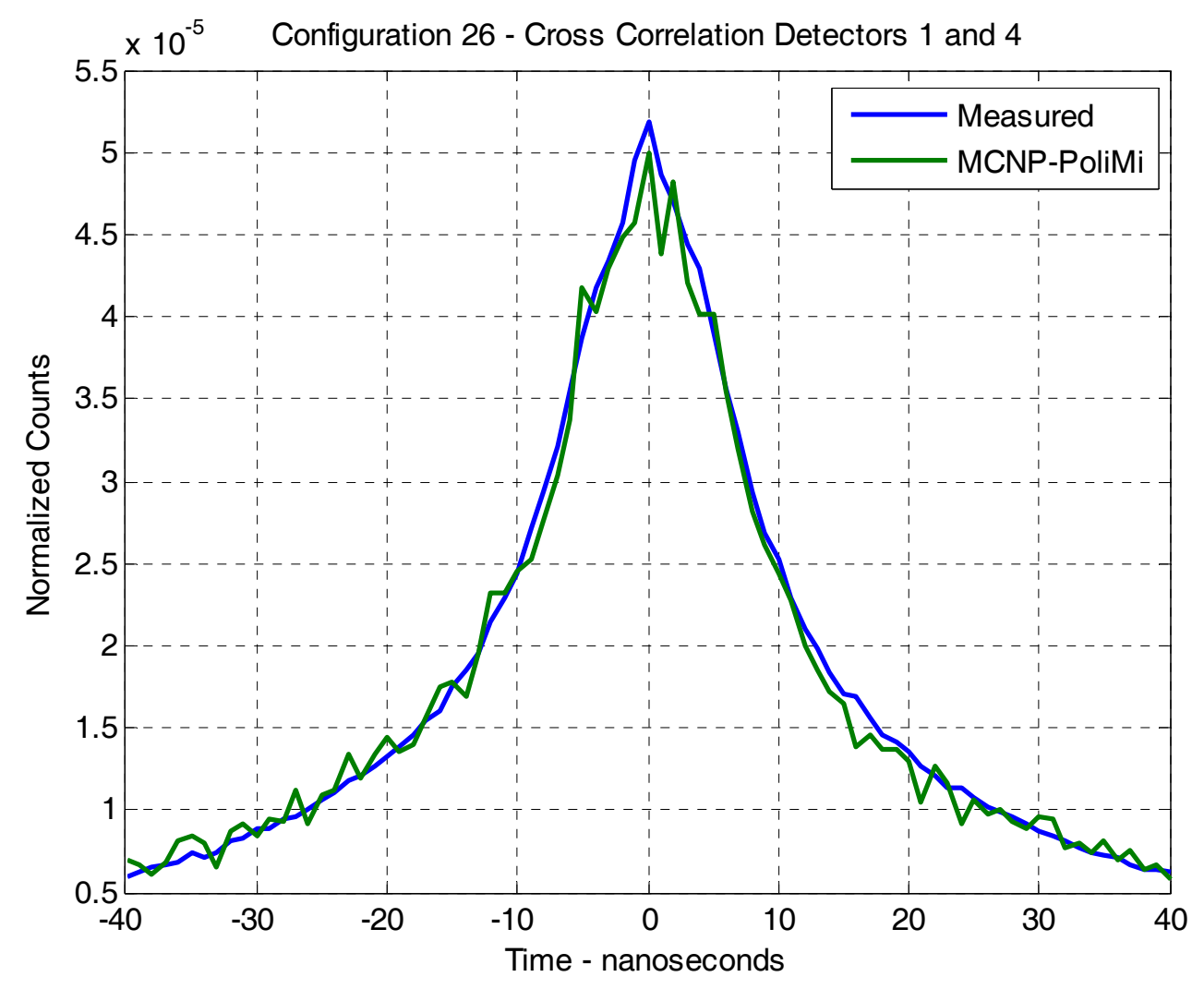

Fig. E.85. Time distribution of counts in detector 4 after a count in detector 1 for $\mathbf{4 0}$ ns for configuration 26. 
APPENDIX F: MONTE CARLO INPUTS FOR SOME SOURCE DETECTOR CASTING CALCULATIONS 


\section{APPENDIX F: MONTE CARLO INPUTS FOR SOME SOURCE DETECTOR CASTING CALCULATIONS}

This appendix provides the MCNP-PoliMi input files used to simulate configuration 1, 3, 4, 12, 20, and 26.

Configuration 1 MCNP-PoliMi input deck:

1 Casting with room CF-in Configuration 1

C casting

$11-18.8 \quad 1-23-4$ imp:n,p=1

C Detector

$82-1.032-8 \quad$ imp:n, $\mathrm{p}=1$ \$ detector

$93-2.70-98 \quad$ imp:n, $\mathrm{p}=1$ \$ Al covering

$104-11.4$-10 $9 \quad$ imp:n,p=1 \$ Pb Covering

11 like 8 but trcl $(8.58258 .58250)$

12 like 9 but trcl $(8.58258 .58250)$

13 like 10 but trcl $(8.58258 .58250)$

14 like 8 but trcl $(17.16500)$

15 like 9 but $\operatorname{trcl}(17.16500)$

16 like 10 but $\operatorname{trcl}(17.16500)$

17 like 8 but trcl $(8.5825-8.58250)$

18 like 9 but trcl $(8.5825-8.58250)$

19 like 10 but $\operatorname{trcl}(8.5825-8.58250)$

C tables

$905-7.82 \quad-90 \quad$ imp:n,p=1

$915-7.82 \quad-91 \quad$ imp:n, $\mathrm{p}=1$

$925-7.82-92 \quad$ imp:n, $p=1$

C floor

$936-2.3 \quad-93 \quad$ imp:n,p=1

$946-2.3 \quad-94 \quad$ imp:n,p=1

$956-2.3 \quad-95 \quad$ imp:n,p=1

$966-2.3 \quad-96 \quad$ imp:n,p=1

$976-2.3 \quad-97 \quad$ imp:n,p=1

$986-2.3 \quad-98 \quad$ imp:n, $\mathrm{p}=1$

$\mathrm{C}$ everything else

$1100-100$ \#1 \#8 \#9 \#10 \#11 \#12 \#13 \#14 \#15 \#16 \#17

\#18 \#19 \#90 \#91 \#92 \#93 \#94 \#95 \#96

\#97 \#98 imp:n,p=1

1110100 imp:n,p=0 \$ Everything Else

C surface Cards

C Casting

$1 \mathrm{cz} 4.445$ \$ Casting IR

$2 \mathrm{cz} 6.35$ \$ Casting OR

3 pz 1.27 \$casting bottom

4 pz 16.51 \$ Casting Top

C Detector 


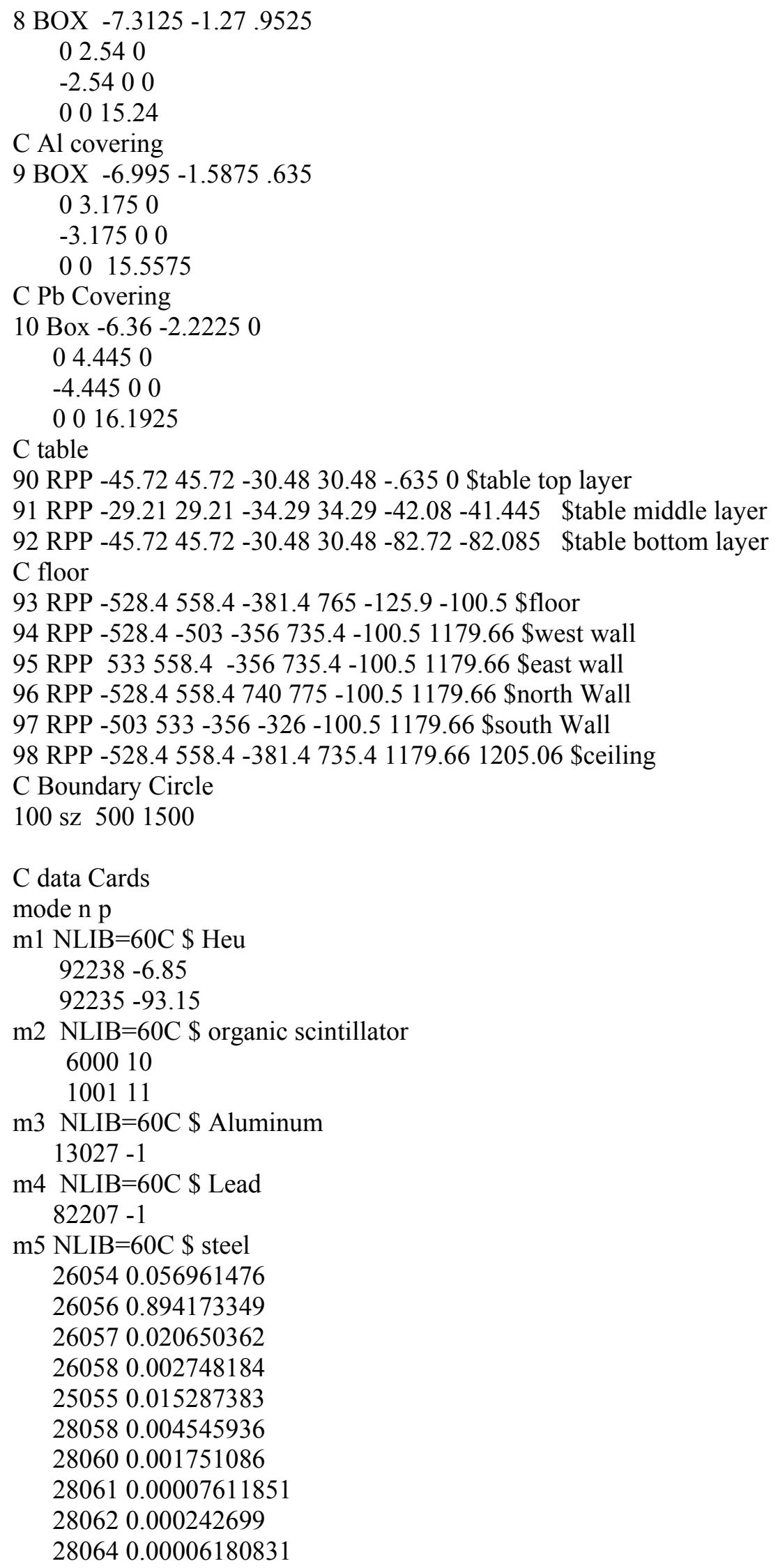


420000.001149575

m6 \$ Concrete Floor

1001.60c .006094

$8016.60 \mathrm{c} .043421$

$14000.60 \mathrm{c} .017390$

13027.60c .001786

$11023.60 \mathrm{c} .000900$

20000.60c .001958

$26000 \quad .000334$

sdef pos $=008.255$

PHYS:N J 20.

PHYS:P 011

CUT:N 100.00000

CUT:P 100.00000 J 0

IDUM $1121 \mathrm{~J} 148111417$

RDUM 0.0001500 .001000

CTME 60000

NPS 15000000

PRDMP J -10

FILES 21 DUMN1 \$ 3J 22 DUMN2 
Configuration 3 MCNP-PoliMi input deck:

1 Casting with room DT generator Configuration 3

C casting

$11-18.8 \quad 1-23-4$ imp:n,p=1

$25-7.82-153-4$ imp:n,p=1

$82-1.032 \quad-8 \quad$ imp:n, $\mathrm{p}=1$ \$ detector

$93-2.70 \quad-98 \quad$ imp:n, $\mathrm{p}=1$ \$ Al covering

$104-11.4-109 \quad$ imp:n, $\mathrm{p}=1$ \$ Pb Covering

11 like 8 but trcl $(8.58258 .58250)$

12 like 9 but trcl $(8.58258 .58250)$

13 like 10 but trcl $(8.58258 .58250)$

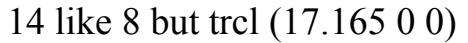

15 like 9 but trcl $(17.16500)$

16 like 10 but $\operatorname{trcl}(17.16500)$

C Tables

$905-7.82 \quad-90 \quad$ imp:n, $p=1$

$915-7.82 \quad-91 \quad$ imp:n, $\mathrm{p}=1$

$925-7.82 \quad-92 \quad$ imp:n,p=1

C floor

$936-2.3 \quad-93 \quad$ imp:n, $p=1$

$946-2.3 \quad-94 \quad$ imp:n, $p=1$

$956-2.3 \quad-95 \quad$ imp:n,p=1

$966-2.3 \quad-96 \quad$ imp:n,p=1

$976-2.3 \quad-97 \quad$ imp:n,p=1

$986-2.3 \quad-98 \quad$ imp:n,p=1

$\mathrm{C}$ everything else

$1100-100$ \#1 \#2 \#8 \#9 \#10 \#11 \#12 \#13 \#14 \#15 \#16

\#90 \#91 \#92 \#93 \#94 \#95 \#96 \#97 \#98 imp:n,p=1

1110100 imp:n,p=0 \$ Everything Else

C surface Cards

C Casting

$1 \mathrm{cz} 4.445$ \$ Casting IR

$2 \mathrm{cz} 6.35$ \$ Casting OR

$3 \mathrm{pz} 1.27$ \$casting bottom

4 pz 16.51 \$ Casting Top

$5 \mathrm{cz} 4.1275$ \$ Can

$6 \mathrm{cz} 6.6675$

C Detector

8 BOX -7.3125 - 1.27 .9525

02.540

$-2.5400$

0015.24

$\mathrm{C}$ Al covering

9 BOX -6.995 - 1.5875 .635

03.1750

$-3.17500$

$\begin{array}{lll}0 & 0 & 15.5575\end{array}$

$\mathrm{C} \mathrm{Pb}$ Covering

10 Box $-6.36-2.22250$ 


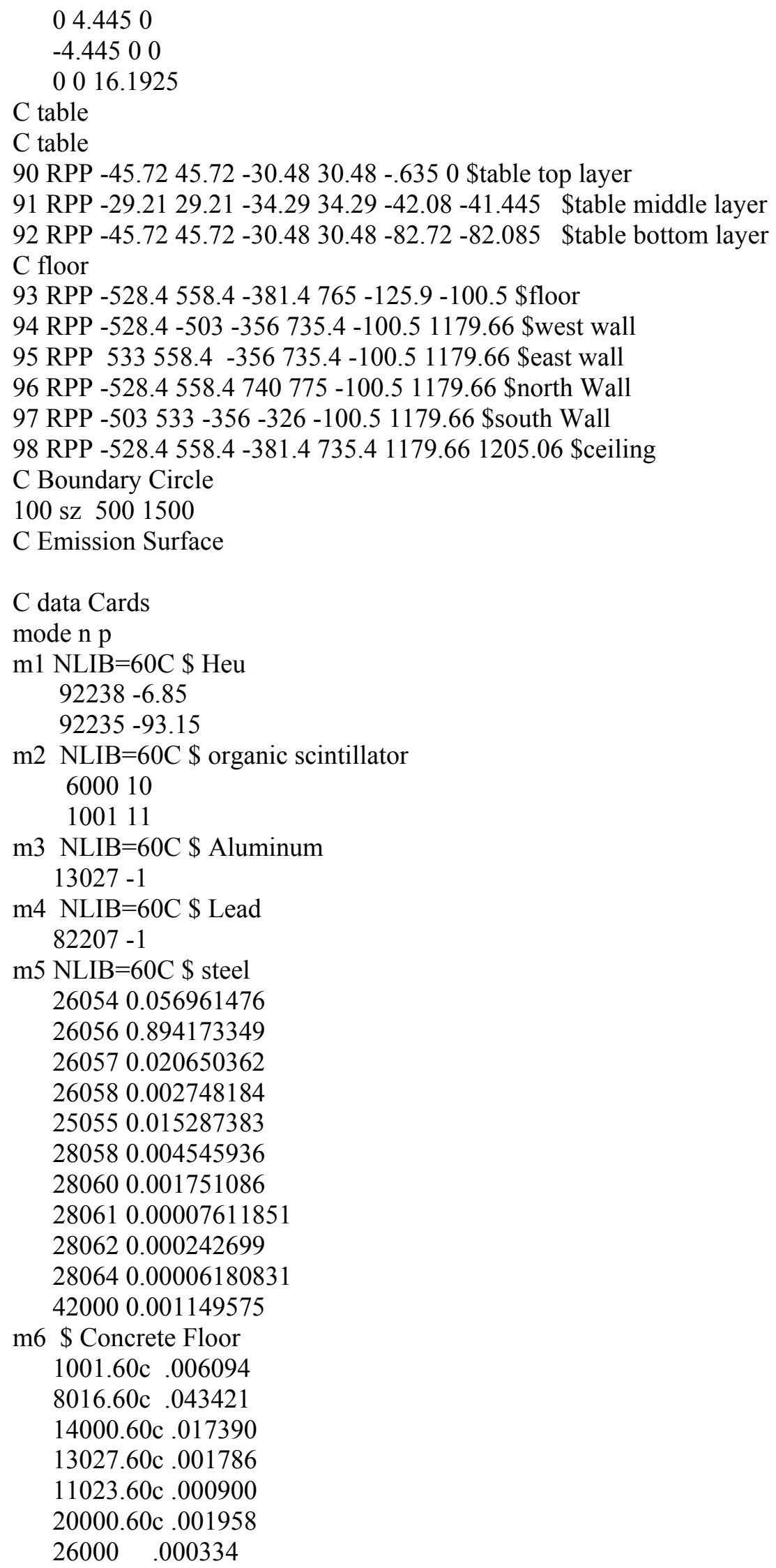


SDEF pos $=0-10.167 .62 \mathrm{ERG}=14.100 \mathrm{VEC}=0.08748910 \mathrm{DIR}=\mathrm{D} 1 \quad \$ 5$ degree offset

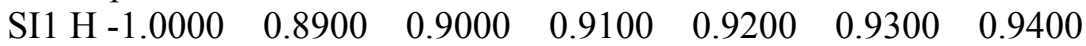

$\begin{array}{llllll}0.9500 & 0.9600 & 0.9700 & 0.9800 & 0.9900 & 1.0000\end{array}$

$\begin{array}{llllllll}\text { SP1 D } & 0 & 0.005596 & 0.0071 & 0.009682 & 0.013375 & 0.029666 & 0.056974\end{array}$

$\begin{array}{lllllll}0.107862 & 0.14031 & 0.152666 & 0.155609 & 0.15865 & 0.16251\end{array}$

PHYS:N J 20.

PHYS:P 011

CUT:N 100.00000

CUT:P $100.00000 \mathrm{~J} 0$

IDUM $0121 \mathrm{~J} 1381114$

RDUM 0.0001500 .001000

NPS 30000000

DBCN 6647299061401 6J 1 4J 76459

CTME 60000

PRDMP J -10 1

FILES 21 DUMN1 \$ 3J 22 DUMN2 
Configuration 4 MCNP-PoliMi input deck:

2 castings with room $\mathrm{CF}$-in Configuration 4

C casting

$11-18.8 \quad 1-23-4$ imp:n,p=1

2 like 1 but trcl (12.72 $0-1.27) \$$ casting 2

C Detector

$82-1.032 \quad-8 \quad$ imp:n, $\mathrm{p}=1$ \$ detector

$93-2.70 \quad-98 \quad$ imp:n, $\mathrm{p}=1$ \$ Al covering

$104-11.4$-10 $9 \quad$ imp:n, $\mathrm{p}=1$ \$ Pb Covering

11 like 8 but trel $(12.700)$

12 like 9 but $\operatorname{trcl}\left(\begin{array}{lll}12.7 & 0 & 0\end{array}\right)$

13 like 10 but $\operatorname{trcl}\left(\begin{array}{lll}12.7 & 0 & 0\end{array}\right)$

14 like 8 but trcl $(0-17.20)$

15 like 9 but $\operatorname{trcl}(0-17.20)$

16 like 10 but $\operatorname{trcl}(0-17.20)$

17 like 8 but trcl (12.7 -17.2 0)

18 like 9 but trcl (12.7 -17.2 0)

19 like 10 but trcl (12.7 -17.2 0)

C tables

$905-7.82 \quad-90 \quad$ imp:n,p=1

$915-7.82 \quad-91 \quad$ imp:n,p=1

$925-7.82 \quad-92 \quad$ imp:n,p=1

C floor

$936-2.3 \quad-93 \quad$ imp:n, $p=1$

$946-2.3 \quad-94 \quad$ imp:n,p=1

$956-2.3 \quad-95 \quad$ imp:n, $\mathrm{p}=1$

$966-2.3 \quad-96 \quad$ imp:n, $\mathrm{p}=1$

$976-2.3 \quad-97 \quad$ imp:n,p=1

$986-2.3 \quad-98 \quad$ imp:n, $\mathrm{p}=1$

$\mathrm{C}$ everything else

$1100-100$ \#1 \#2 \#8 \#9 \#10 \#11 \#12 \#13 \#14 \#15 \#16 \#17

\#18 \#19 \#90 \#91 \#92 \#93 \#94 \#95 \#96

\#97 \#98 imp:n,p=1

1110100 imp:n,p=0 \$ Everything Else

C surface Cards

C Casting

$1 \mathrm{cz} 4.445$ \$ Casting IR

$2 \mathrm{cz} 6.35$ \$ Casting OR

3 pz 1.27 \$casting bottom

$4 \mathrm{pz} 16.51$ \$ Casting Top

C Detector

$\begin{array}{llll}8 \text { Box } & 1.2700 & 7.3525 & 0.9525\end{array}$

$\begin{array}{lll}0 & 2.5400 & 0\end{array}$

$\begin{array}{lll}-2.5400 & 0 & 0\end{array}$

$\begin{array}{lll}0 & 0 & 15.2400 \$ \text { Detector } 1\end{array}$

$\begin{array}{llll}9 \text { BOX } & 1.5875 & 7.0350 & 0.6350\end{array}$

$\begin{array}{lll}0 & 3.1750 & 0\end{array}$

$\begin{array}{lll}-3.1750 & 0 & 0\end{array}$

$\begin{array}{lll}0 & 0 & 15.5575 \$ \mathrm{Al} \text { Covering } 1\end{array}$ 


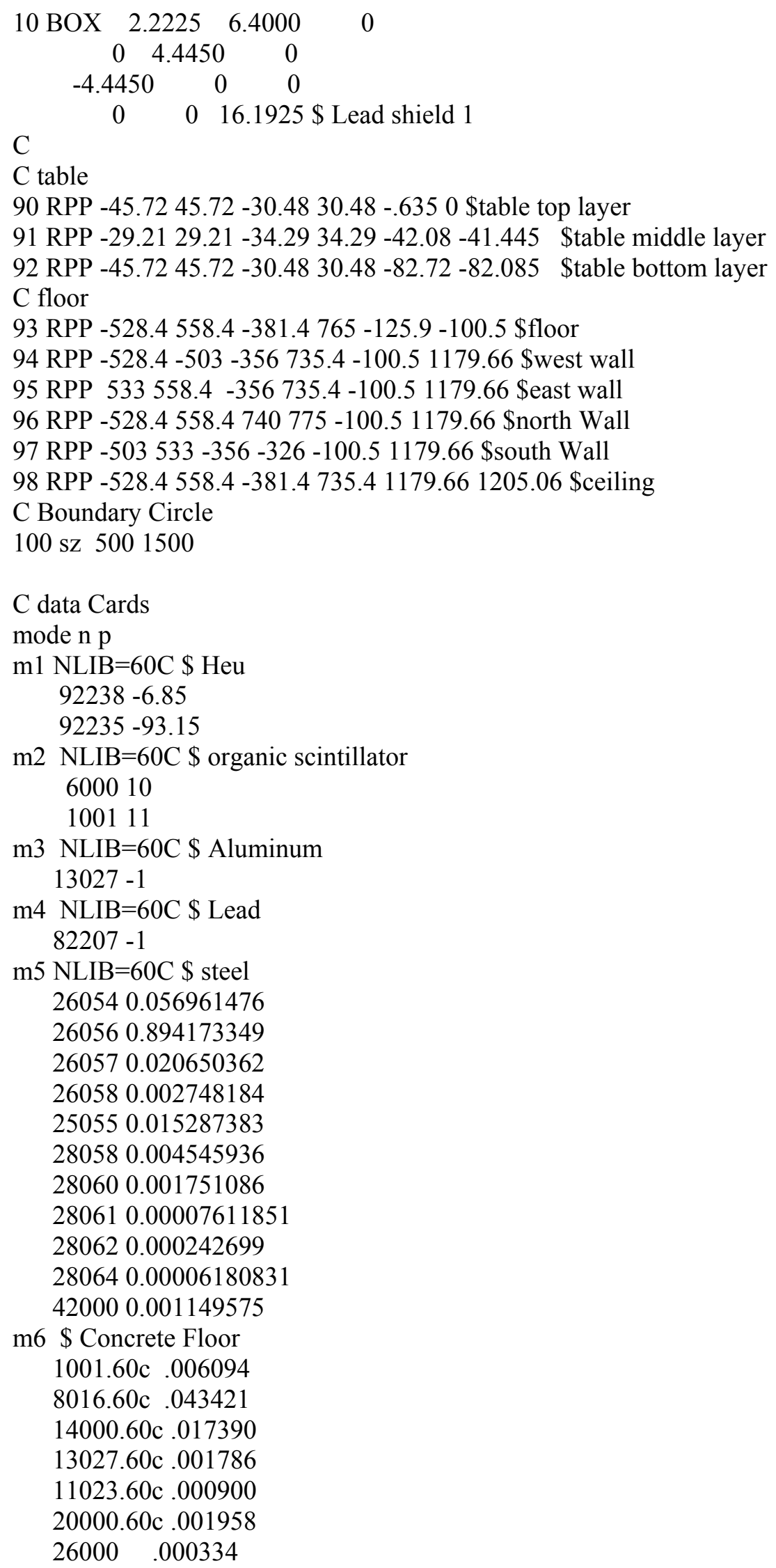


sdef pos= 008.255

PHYS:N J 20.

PHYS:P 011

CUT:N 100.00000

CUT:P 100.00000 J 0

IDUM $1121 \mathrm{~J} 148111417$

RDUM 0.0001500 .001000

CTME 60000

NPS 30000000

PRDMP J -10

FILES 21 DUMN1 \$ 3J 22 DUMN2 
Configuration 12 MCNP-PoliMi input deck:

3 castings with room CF-in Configuration 12

C casting

$11-18.8 \quad 1-23-4$ imp:n,p=1

2 like 1 but trcl $(12.720-1.27) \$$ casting 2

3 like 2 but trcl (6.36 11.0 -1.27) \$ casting3

C Detector

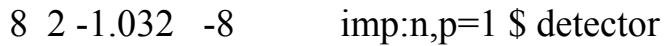

$93-2.70 \quad-98 \quad$ imp:n, $\mathrm{p}=1$ \$ Al covering

$104-11.4-109 \quad$ imp:n, $\mathrm{p}=1$ \$ Pb Covering

$112-1.032-11 \quad$ imp:n,p=1 \$ detector

$123-2.70 \quad-1211 \quad$ imp: $n, p=1$ \$ Al covering

$134-11.4-1312 \quad$ imp: $n, p=1$ \$ Pb Covering

$142-1.032-14 \quad$ imp:n, $p=1$ \$ detector

$153-2.70 \quad-1514 \quad$ imp:n,p=1 \$ Al covering

$164-11.4-1615 \quad$ imp:n, $\mathrm{p}=1$ \$ Pb Covering

$172-1.032-17 \quad$ imp:n, $\mathrm{p}=1$ \$ detector

$183-2.70 \quad-1817 \quad$ imp:n, $p=1$ \$ Al covering

$194-11.4 \quad-1918 \quad$ imp:n,p=1 \$ Pb Covering

C tables

$905-7.82 \quad-90 \quad$ imp:n,p=1

$915-7.82 \quad-91 \quad$ imp:n,p=1

$925-7.82 \quad-92 \quad$ imp:n,p=1

C floor

$93 \quad 6-2.3 \quad-93 \quad$ imp:n, $p=1$

$946-2.3 \quad-94 \quad$ imp:n,p=1

$956-2.3 \quad-95 \quad$ imp:n, $\mathrm{p}=1$

$966-2.3 \quad-96 \quad$ imp:n,p=1

$976-2.3 \quad-97 \quad$ imp:n,p=1

$986-2.3 \quad-98 \quad$ imp:n,p=1

$\mathrm{C}$ everything else

$1100-100$ \#1 \#2 \#3 \#8 \#9 \#10 \#11 \#12 \#13 \#14 \#15 \#16 \#17

\#18 \#19 \#90 \#91 \#92 \#93 \#94 \#95 \#96

\#97 \#98 imp:n,p=1

1110100 imp:n,p=0 \$ Everything Else

C surface Cards

C Casting

$1 \mathrm{cz} 4.445 \$$ Casting IR

$2 \mathrm{cz} 6.35$ \$ Casting OR

$3 \mathrm{pz} 1.27$ \$casting bottom

4 pz 16.51 \$ Casting Top

C Detector

\begin{tabular}{cccc}
8 Box & -5.85 & -4.536438418 & 0.9525 \\
-1.796051224 & -1.796051224 & 0 \\
1.796051224 & -1.796051224 & 0 \\
0 & 0 & \multicolumn{2}{c}{$15.24 \$$ Detector 1} \\
9 BOX & -5.85 & -4.087425612 & 0.635 \\
-2.24506403 & -2.24506403 & 0 \\
2.24506403 & -2.24506403 & 0
\end{tabular}




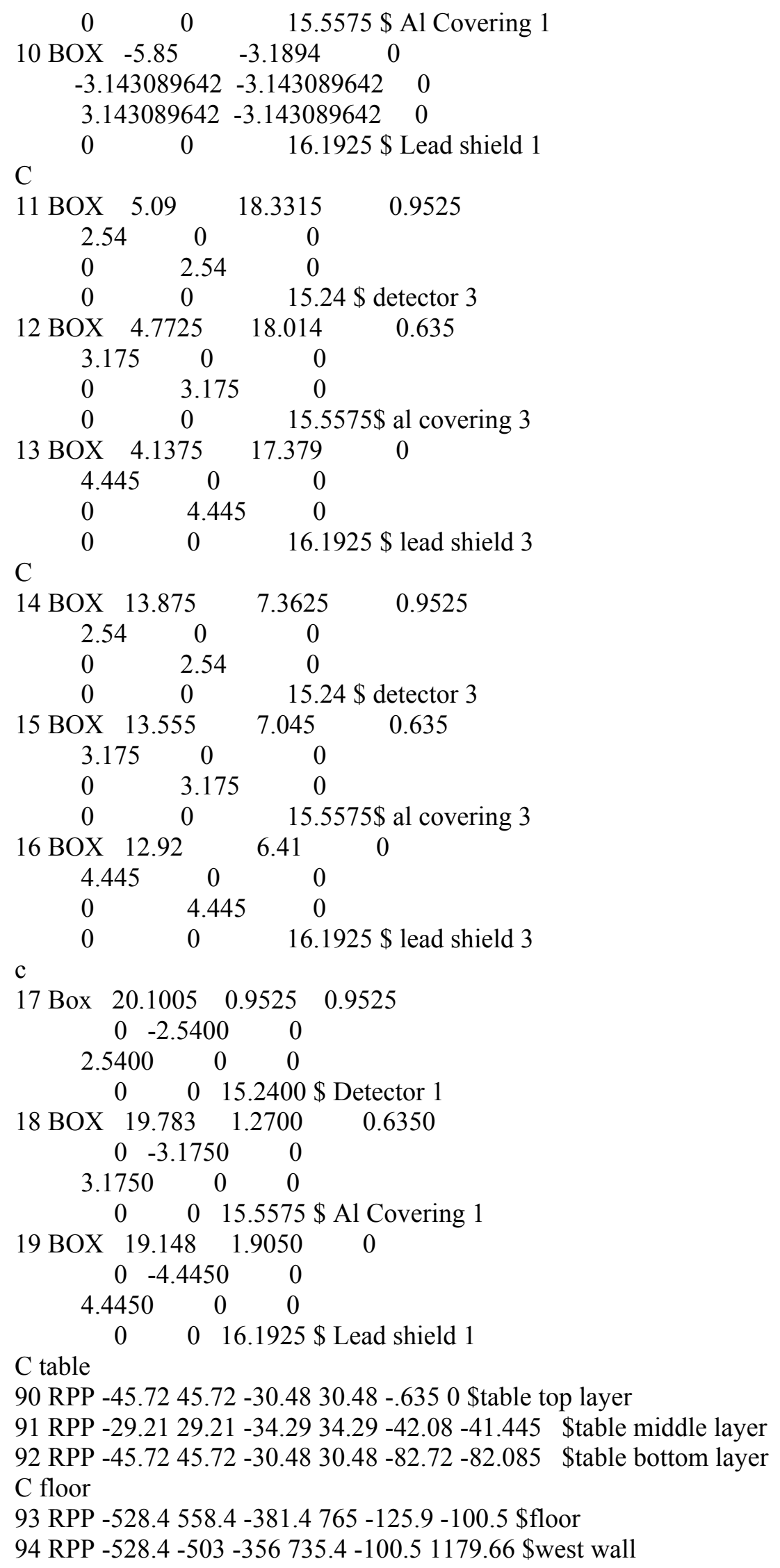


95 RPP $533558.4-356735.4-100.51179 .66$ Seast wall 96 RPP -528.4 558.4 740 775 -100.5 1179.66 \$north Wall 97 RPP -503 533 -356 -326 -100.5 1179.66 \$south Wall 98 RPP -528.4 558.4 -381.4 735.4 1179.66 1205.06 \$ceiling C Boundary Circle $100 \mathrm{sz} 5001500$

C data Cards

mode $\mathrm{n} p$

$\mathrm{m} 1 \mathrm{NLIB}=60 \mathrm{C} \$ \mathrm{Heu}$ $92238-6.85$

$92235-93.15$

$\mathrm{m} 2 \mathrm{NLIB}=60 \mathrm{C} \$$ organic scintillator 600010 100111

m3 NLIB $=60 \mathrm{C} \$$ Aluminum $13027-1$

$\mathrm{m} 4 \mathrm{NLIB}=60 \mathrm{C} \$$ Lead $82207-1$

m5 NLIB $=60 \mathrm{C} \$$ steel 260540.056961476 260560.894173349 260570.020650362 260580.002748184 250550.015287383 280580.004545936 280600.001751086 280610.00007611851 280620.000242699 280640.00006180831 420000.001149575

m6 \$ Concrete Floor 1001.60c .006094 8016.60c .043421 $14000.60 \mathrm{c} .017390$ $13027.60 \mathrm{c} .001786$ $11023.60 \mathrm{c} .000900$ $20000.60 \mathrm{c} .001958$ $26000 \quad .000334$

sdef pos $=008.255$

PHYS:N J 20.

PHYS:P 011

CUT:N 100.00000

CUT:P 100.00000 J 0

IDUM $1121 \mathrm{~J} 148111417$

RDUM 0.0001500 .001000

CTME 60000

NPS 30000000

PRDMP J -10

FILES 21 DUMN1 \$ 3J 22 DUMN2 
Configuration 20 MCNP-PoliMi input deck:

4 castings with room, $\mathrm{CF}$ - in Configuration 20

C casting

$11-18.8 \quad 1-23-4$ imp:n,p=1

2 like 1 but trcl (12.72 $0-1.27) \$$ casting2

3 like 1 but trcl (-6.36 11.0 -1.27) \$ casting3

4 like 1 but trcl (6.36 $11.0-1.27)$ \$ casting4

C Detector

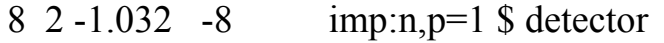

$9 \quad 3-2.70 \quad-98 \quad$ imp:n, $p=1$ \$ Al covering

$104-11.4-109 \quad$ imp:n,p=1 \$ Pb Covering

$112-1.032-11 \quad$ imp:n, $\mathrm{p}=1$ \$ detector

$123-2.70 \quad-1211 \quad$ imp:n, $\mathrm{p}=1$ \$ Al covering

$134-11.4-1312 \quad$ imp: $n, p=1$ \$ Pb Covering

$142-1.032-14 \quad$ imp:n, $\mathrm{p}=1$ \$ detector

$153-2.70-1514 \quad$ imp: $n, p=1$ \$ Al covering

$164-11.4-1615 \quad$ imp:n, $\mathrm{p}=1$ \$ Pb Covering

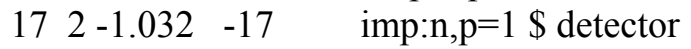

$\begin{array}{lllll}18 & 3 & -2.70 & -18 & 17\end{array}$ imp:n, $\mathrm{p}=1$ \$ Al covering

$194-11.4$-19 $18 \quad$ imp:n,p=1 \$ Pb Covering

C tables

$905-7.82 \quad-90 \quad$ imp:n,p=1

$915-7.82 \quad-91 \quad$ imp:n, $\mathrm{p}=1$

$925-7.82 \quad-92 \quad$ imp:n, $p=1$

C floor

$936-2.3 \quad-93 \quad$ imp:n, $p=1$

$946-2.3 \quad-94 \quad$ imp:n, $p=1$

$956-2.3 \quad-95 \quad$ imp:n, $\mathrm{p}=1$

$966-2.3 \quad-96 \quad$ imp:n,p=1

$976-2.3 \quad-97 \quad$ imp:n, $p=1$

$986-2.3 \quad-98 \quad$ imp:n, $\mathrm{p}=1$

$\mathrm{C}$ everything else

$1100-100$ \#1 \#2 \#3 \#4 \#8 \#9 \#10 \#11 \#12 \#13

\#14 \#15 \#16 \#17 \#18 \#19 \#90

\#91 \#92 \#93 \#94 \#95 \#96 \#97 \#98 imp:n,p=1

1110100 imp:n,p=0 \$ Everything Else

C surface Cards

C Casting

$1 \mathrm{cz} 4.445$ \$ Casting IR

$2 \mathrm{cz} 6.35$ \$ Casting OR

$3 \mathrm{pz} 1.27$ \$casting bottom

$4 \mathrm{pz} 16.51$ \$ Casting Top

C Detector

$\begin{array}{lllll}8 & \text { BOX } & -7.13189 & 3.53414 & 0.9525\end{array}$

$\begin{array}{lll}-2.53033 & 0.221376 \quad 0\end{array}$

$-0.22138-2.53033 \quad 0$

$\begin{array}{lll}0 & 0 & 15.24 \$ \text { detector } 3\end{array}$

9 BOX $-6.78793 \quad 3.82276 \quad 0.635$

$\begin{array}{llll}-3.16292 & 0.276719 & 0\end{array}$ 


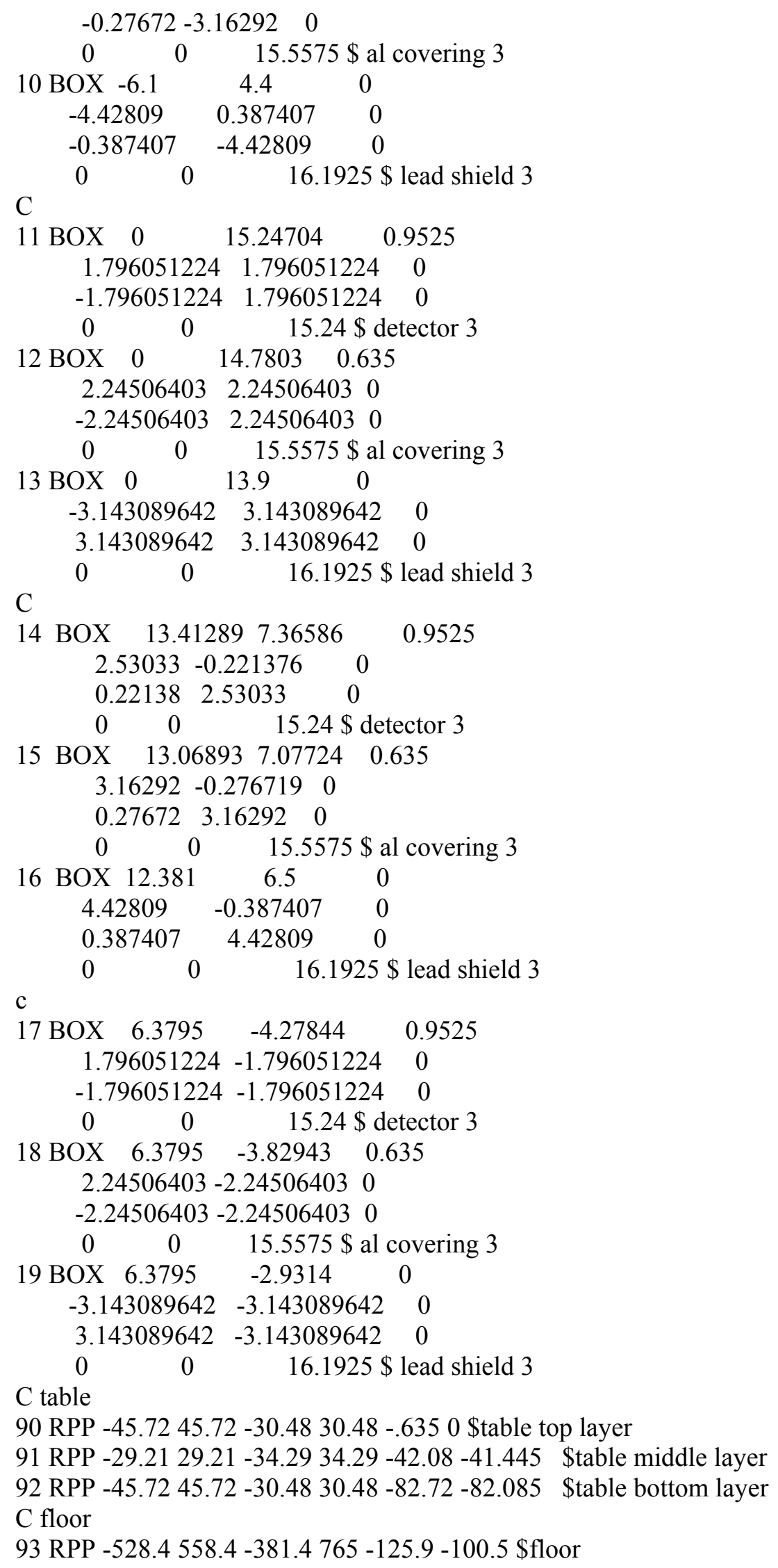


94 RPP -528.4 -503 -356 735.4 -100.5 1179.66 \$west wall 95 RPP $533558.4-356735.4-100.51179 .66$ \$east wall 96 RPP -528.4 558.4 740 $775-100.51179 .66$ \$north Wall 97 RPP -503 533 -356 -326 -100.5 1179.66 \$south Wall 98 RPP -528.4 558.4 -381.4 735.4 1179.66 1205.06 \$ceiling C Boundary Circle $100 \mathrm{sz} 5001500$

C data Cards

mode $\mathrm{n} p$

$\mathrm{m} 1 \mathrm{NLIB}=60 \mathrm{C} \$ \mathrm{Heu}$ $92238-6.85$ $92235-93.15$

$\mathrm{m} 2 \mathrm{NLIB}=60 \mathrm{C} \$$ organic scintillator 600010 100111

$\mathrm{m} 3 \mathrm{NLIB}=60 \mathrm{C} \$$ Aluminum $13027-1$

$\mathrm{m} 4 \mathrm{NLIB}=60 \mathrm{C} \$$ Lead $82207-1$

m5 NLIB $=60 \mathrm{C} \$$ steel 260540.056961476 260560.894173349 260570.020650362 260580.002748184 250550.015287383 280580.004545936 280600.001751086 280610.00007611851 280620.000242699 280640.00006180831 420000.001149575

m6 \$ Concrete Floor 1001.60c .006094 8016.60c .043421 $14000.60 \mathrm{c} .017390$ $13027.60 \mathrm{c} .001786$ $11023.60 \mathrm{c} .000900$ 20000.60 c .001958 $26000 \quad .000334$

sdef pos $=008.255$

PHYS:N J 20.

PHYS:P 011

CUT:N 100.00000

CUT:P $100.00000 \mathrm{~J} 0$

IDUM $1121 \mathrm{~J} 148111417$

RDUM 0.0001500 .001000

CTME 60000

NPS 30000000

PRDMP J -10

FILES 21 DUMN1 \$ 3J 22 DUMN2 
Configuration 26 MCNP-PoliMi input deck:

5 castings with room CF-in Configuration 26

C casting

$11-18.8 \quad 1-23-4$ imp:n,p=1

2 like 1 but trcl (12.72 $0-1.27)$ \$ casting2

3 like 1 but trcl (-12.72 $0-1.27)$ \$ casting3

4 like 1 but trcl (-6.36 11.0 -1.27) \$ casting4

5 like 1 but trcl (6.36 11.0 -1.27) \$ casting5

C Detector

$\begin{array}{lllll}8 & 2 & -1.032 & -8 & \text { imp:n, } \mathrm{p}=1 \text { \$ detector }\end{array}$

$93 \quad-2.700 \quad-98 \quad$ imp:n, $\mathrm{p}=1$ \$ Al covering

$104-11.400-109$ imp:n,p=1 \$ Pb Covering

$112-1.032-11 \quad$ imp:n, $\mathrm{p}=1$ \$ detector

$123-2.700-1211 \quad$ imp:n,p=1 \$ Al covering

$134-11.400-1312 \quad$ imp:n, $\mathrm{p}=1$ \$ Pb Covering

$142-1.032-14 \quad$ imp:n, $p=1$ \$ detector

$153-2.700-1514 \quad$ imp:n, $\mathrm{p}=1$ \$ Al covering

$164-11.400-1615 \quad$ imp:n, $\mathrm{p}=1$ \$ Pb Covering

$172-1.032-17 \quad$ imp:n, $\mathrm{p}=1$ \$ detector

$183-2.700-1817 \quad$ imp:n,p=1 \$ Al covering

$194-11.400-1918 \quad$ imp:n, $\mathrm{p}=1$ \$ Pb Covering

C tables

$905-7.82 \quad-90 \quad$ imp:n,p=1

$915-7.82 \quad-91 \quad$ imp:n, $\mathrm{p}=1$

$925-7.82 \quad-92 \quad$ imp:n,p=1

C floor

$936-2.3 \quad-93 \quad$ imp:n, $\mathrm{p}=1$

$946-2.3 \quad-94 \quad$ imp:n,p=1

$956-2.3 \quad-95 \quad$ imp:n, $p=1$

$966-2.3 \quad-96 \quad$ imp:n,p=1

$976-2.3 \quad-97 \quad$ imp:n,p=1

$986-2.3 \quad-98 \quad$ imp:n, $\mathrm{p}=1$

$\mathrm{C}$ everything else

$1100-100$ \#1 \#2 \#3 \#4 \#5 \#8 \#9 \#10 \#11 \#12 \#13 \#14

\#15 \#16 \#17 \#18 \#19 \#90 \#91 \#92 \#93 \#94

\#95 \#96 \#97 \#98 imp:n,p=1

1110100 imp:n,p=0 \$ Everything Else

C surface Cards

C Casting

$1 \mathrm{cz} 4.445 \$$ Casting IR

$2 \mathrm{cz} 6.35$ \$ Casting OR

3 pz 1.27 \$casting bottom

$4 \mathrm{pz} 16.51$ \$ Casting Top

C Detector

8 BOX $-6.0853 \quad-4.64704 \quad 0.9525$

$1.796051224-1.796051224 \quad 0$

$\begin{array}{lll}-1.796051224 & -1.796051224 & 0\end{array}$

$\begin{array}{lll}0 & 0 & 15.24 \$ \text { detector } 4\end{array}$

$\begin{array}{llll}9 \mathrm{BOX} & -6.0853 & -4.19803 & 0.635\end{array}$ 


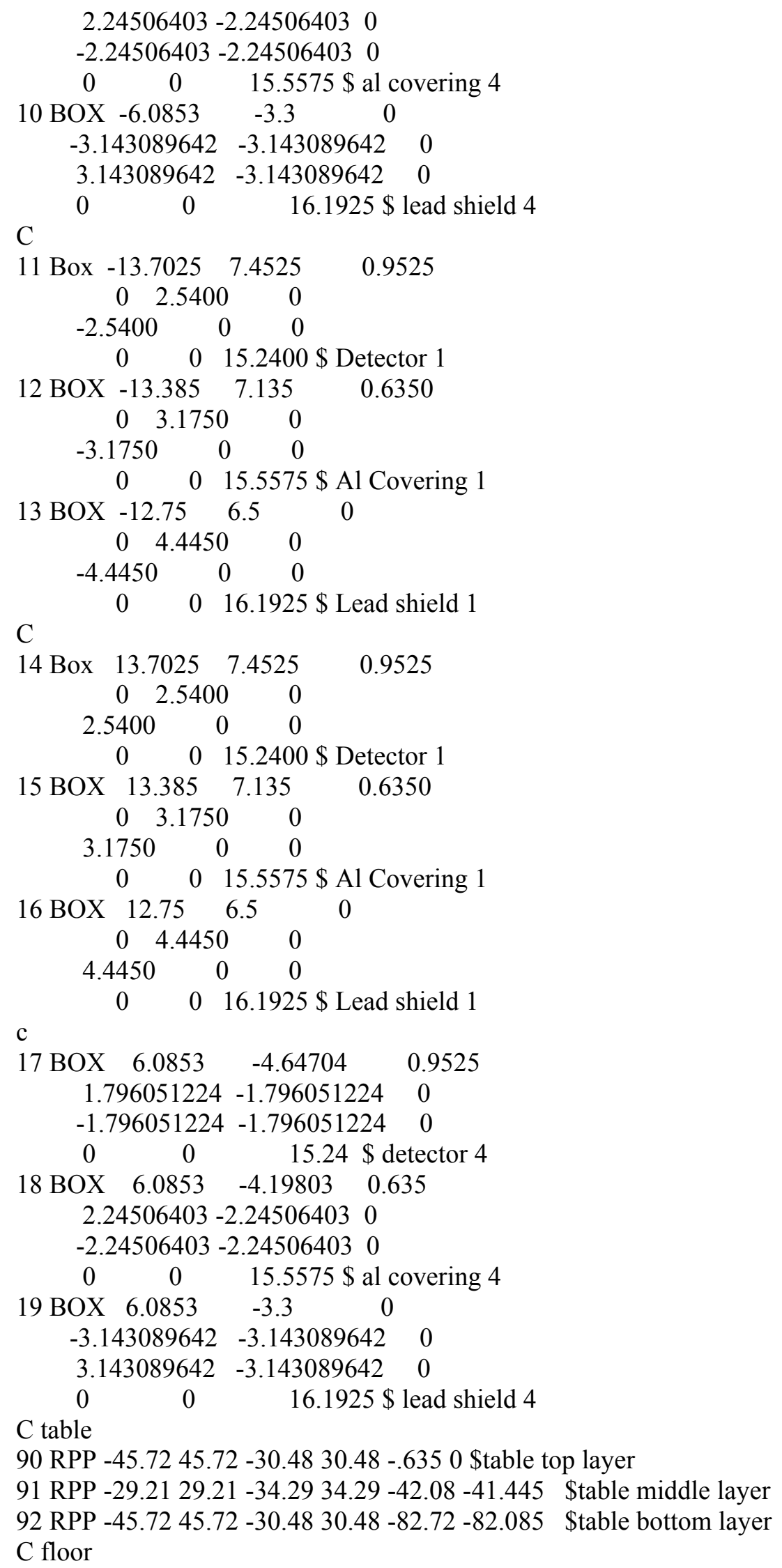


93 RPP -528.4 $558.4-381.4765-125.9-100.5$ \$floor 94 RPP -528.4 -503 -356 735.4 -100.5 1179.66 \$west wall 95 RPP $533558.4-356735.4-100.51179 .66$ \$east wall 96 RPP -528.4 558.4 740 775 -100.5 1179.66 \$north Wall 97 RPP -503 533 -356 -326 -100.5 1179.66 \$south Wall 98 RPP -528.4 558.4 -381.4 735.4 1179.66 1205.06 \$ceiling C Boundary Circle $100 \mathrm{sz} 5001500$

C data Cards

mode $\mathrm{n} p$

$\mathrm{m} 1 \mathrm{NLIB}=60 \mathrm{C} \$ \mathrm{Heu}$ $92238-6.85$ $92235-93.15$

$\mathrm{m} 2 \mathrm{NLIB}=60 \mathrm{C} \$$ organic scintillator 600010 100111

m3 NLIB $=60 \mathrm{C} \$$ Aluminum $13027-1$

$\mathrm{m} 4 \mathrm{NLIB}=60 \mathrm{C} \$$ Lead $82207-1$

m5 NLIB $=60 \mathrm{C} \$$ steel 260540.056961476 260560.894173349 260570.020650362 260580.002748184 250550.015287383 280580.004545936 280600.001751086 280610.00007611851 280620.000242699 280640.00006180831 420000.001149575

m6 \$ Concrete Floor 1001.60c .006094 8016.60c .043421 14000.60c .017390 13027.60c .001786 $11023.60 \mathrm{c} .000900$ 20000.60c .001958 $26000 \quad .000334$ 
sdef pos= 008.255

PHYS:N J 20.

PHYS:P 011

CUT:N 100.00000

CUT:P 100.00000 J 0

IDUM $1121 \mathrm{~J} 148111417$

RDUM 0.0001500 .001000

CTME 60000

NPS 15000000

PRDMP J -10

FILES 21 DUMN1 \$ 3J 22 DUMN2 